\title{
VEGF-mediated vascular functions in health and disease
}

\section{Ziquan Cao}

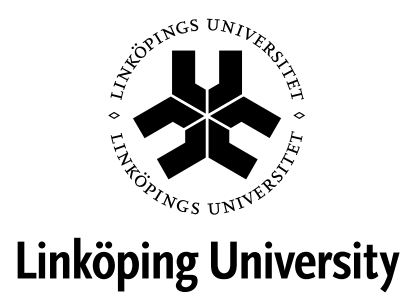

Department of Medical and Health Sciences

Linköping University, Sweden

Linköping 2015 
VEGF-mediated vascular functions in health and disease

(C) Ziquan Cao, 2015

Cover/Illustration/Design: Ziquan Cao and LiU-Tryck.

Published article has been reprinted with the permission of the copyright holder.

Printed in Sweden by LiU-Tryck, Linköping, Sweden, 2015

ISBN 978-91-7519-079-2

ISSN 0345-0082 


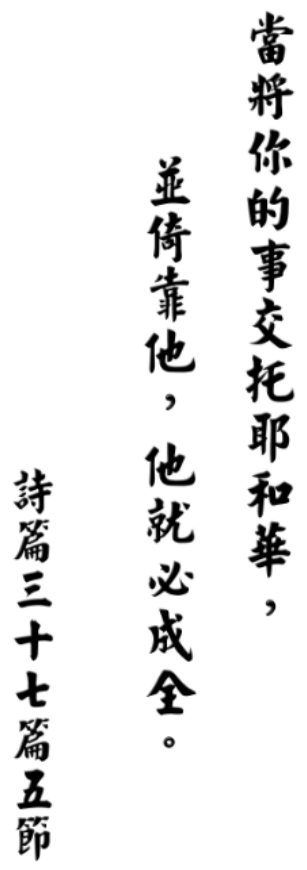





\section{VEGF-MEDIATED VASCULAR FUNCTIONS \\ IN HEALTH AND DISEASE}

\section{THESIS FOR DOCTORAL DEGREE ( Ph.D. )}

By

\section{Ziquan Cao}

Thesis defense

Date and time: 13:00 on Monday, May 25, 2015

Venue: Aulan, Nilsholger, Linköping University

\section{Principal Supervisor:}

Professor Eric Wahlberg

Linköping University

Department of Medical and Health

Sciences

Co-supervisors:

Professor Yihai Cao

Karolinska Institutet

Department of Microbiology, Tumor and Cell Bioloigy

Professor Toste Länne

Linköping University

Department of Medical and Health

Sciences

Doctor Kayoko Hosaka

Karolinska Institutet

Department of Microbiology, Tumor and Cell Bioloigy
Opponent:

Professor Jack L. Arbiser

Emory University School of Medicine

Department of Dematology

Examination Board:

Associate Professor Jordi Altimiras

Linköping University

Department of Physics, Chemistry and

Biology

Associate Professor Jenny L Persson

Lund University

Department of Laboratory Medicine

Division of Experimental Cancer Research

Associate Professor Lennart Nilsson

Linköping University

Department of Medical and Health

Sciences 



\section{TABLE OF CONTENTS}

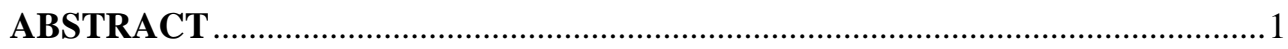

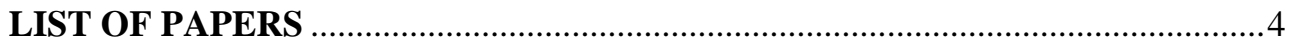

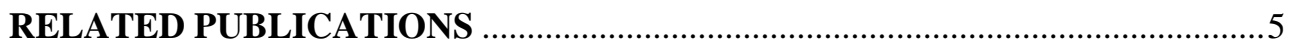

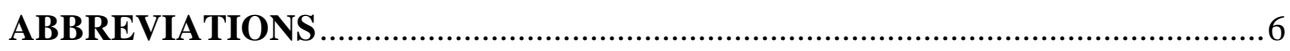

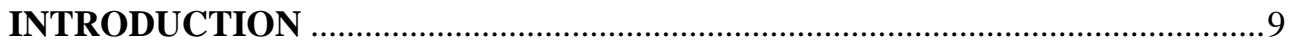

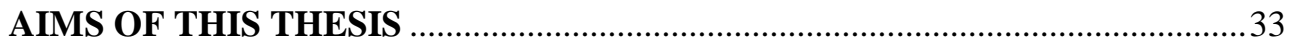

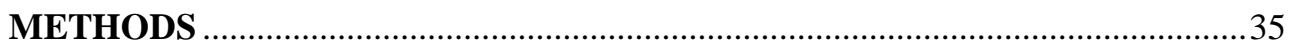

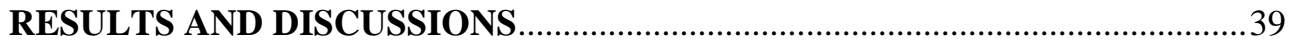

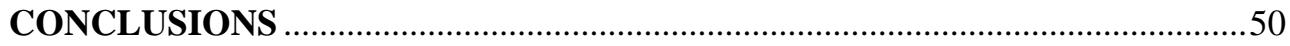

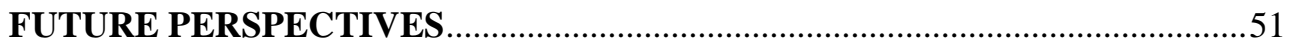

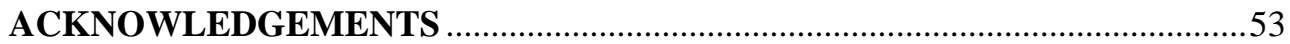

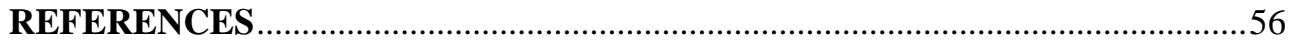





\section{ABSTRACT}

Angiogenesis is essential for physiological processes including embryonic development, tissue regeneration, and reproduction. Under various pathological conditions the same angiogenic process contribute to the onset, development, and progression of many human diseases including cancer, diabetic complications, ocular disease, chronic inflammation and cardiovascular disease. Vascular endothelial growth factor (VEGF) is a key angiogenic factor for physiological and pathological angiogenesis. In addition to its strong angiogenic activity, VEGF also potently induces vascular permeability, often causing tissue edema in various pathological tissues. VEGF transduces its vascular signal through two tyrosine kinase receptors-VEGFR1 and VEGFR2, the latter being a functional receptor that mediates both angiogenic and vascular permeability effects. To study physiological and pathological functions of VEGF, we developed novel zebrafish disease models that permit us to study hypoxia-induced retinopathy and cancer metastasis processes. We have also administered anti-VEGF and antiVEGFR specific antibodies to healthy mice to study the homeostatic role of VEGF in the maintenance of vascular integrity and its functions in various tissues and organs. Finally, using a zebrafish model, we evaluated if VEGF expression is regulated by circadian clock genes. In paper I, we developed protocols that create hypoxia-induced retinopathy in adult zebrafish. Adult fli1:EGFP zebrafish were placed in hypoxic water for 3-10 days with retinal neovascularization being analyzed using confocal microscopy. This model provides a unique opportunity to kinetically study the development of retinopathy in adult animals using non-invasive protocols and to assess the therapeutic efficacy of orally administered anti-angiogenic drugs. In paper II, we developed a zebrafish metastasis model to dissect the complex events of hypoxia-induced tumor cell invasion and metastasis in association with angiogenesis at the single-cell level. In this model, fluorescent DiI-labeled human or mouse tumor cells were implanted into the perivitelline cavity of 48-hour-old zebrafish embryos, which were subsequently placed in hypoxic water for 3 days. Tumor cell invasion, metastasis and pathological angiogenesis were analyzed using fluorescent microscopy in the living fish. The average experimental time for this model is 7 days. Our protocol offers an opportunity to study molecular mechanisms of hypoxia-induced cancer metastasis. In paper III, we show that systemic delivery 
of an anti-VEGF or an anti-VEGF receptor (VEGFR)-2 neutralizing antibody cause global vascular regression in mice. Among all examined tissues, the vasculature in endocrine glands, intestinal villi, and the uterus are most affected in response to VEGF or VEGFR-2 blockades. Pro-longed anti-VEGF treatment resulted in a significant decrease in the circulating levels of the predominant thyroid hormone, free thyroxine, but not the minimal isoform of triiodothyronine, suggesting that chronic anti-VEGF treatment impairs thyroid function. These findings provide structural and functional bases of anti-VEGF-specific druginduced side effects in relation to vascular changes in healthy tissues. In paper IV, we show that disruption of the circadian clock by constant exposure to light coupled with genetic manipulation of key genes in the zebrafish led to impaired developmental angiogenesis. A bmall-specific morpholino inhibited developmental angiogenesis in zebrafish embryos without causing obvious nonvascular phenotypes. Conversely, a period2 morpholino accelerated angiogenic vessel growth, suggesting that Bmal1 and Period2 display opposing angiogenic effects. These results offer mechanistic insights into the role of the circadian clock in regulation of developmental angiogenesis, and our findings may be reasonably extended to other types of physiological or pathological angiogenesis. Overall, the results in this thesis provide further insight to angiogenic mechanistic properties in tissues and suggest possible novel therapeutic targets for the treatment of various angiogenesis-dependent diseases.

Blodkärlsnybildning, så kallad angiogenes, är viktigt för fysiologiska processer vid embryonal utveckling, vävnadsregenerering och reproduktion. Samma angiogena process kan också under olika sjukdomstillstånd bidra till uppkomst, utveckling och progress av många sjukdomar, såsom cancer, diabeteskomplikationer, ögonsjukdomar, kronisk inflammation samt hjärtkärlsjukdom. Vascular endothelial growth factor (VEGF) är mycket viktig för fysiologisk och patologisk angiogenes. Utöver sin starka angiogena effekt inducerar VEGF även ökad kärlpermeabilitet, som ofta orsakar ödem. VEGF utövar sin effekt på kärlen via två tyrosinkinasreceptorer: VEGFR1 och VEGFR2, där den senare är en funktionell receptor som förmedlar både angiogena signaler och har effekter på vaskulär permeabilitet. För att öka möjlgheterna att studera fysiologiska och patologiska funktioner av VEGF, har vi utvecklat sjukdomsmodeller i zebrafisk - hypoxi-inducerad retinopati och metastasering av cancer. Vi har också givit anti-VEGF och anti-VEGFR-specifika antikroppar till friska möss för att utvärdera VEGFs roll vid stabiliseringen av kärlfunktionen i olika vävnader och organ. 
Slutligen,utvärderade vi om expressionen av VEGF regleras av dygnsrytmen genom så kallade klock-gener. I papper I utvecklade vi en modell för hypoxiinducerad retinopati hos vuxna zebrafiskar. Vuxna flil:EGFP zebrafiskar placeras i syrefattigt vatten i 3-10 dagar, varpå retinal nybildning av kärl analyserades. Denna modell ger en unik icke-invasiv möjlighet att studera kinetiskt utveckling av retinopati och den möjliggör bedömning av terapeutiska effekter av oralt givna anti-angiogena läkemedel. I papper II utvecklade vi en zebrafiskmodell för utvärdering av cancermetastasering, som möjliggör studier av detaljerade delprocesser vid hypoxi-inducerad tumörcellsinvasion och metastasering i samband med angiogenes på encellig nivå. I denna modell användes fluorescerande Dil-märkta humana- eller mustumörceller som implanterades vid den perivitellina hålighet hos 48-h-gamla zebrafiskembryon placerade i syrefattigt vatten i 3 dagar. Tumörcellinvasion, metastasering och patologisk angiogenes analyserades med mikroskopi i levande fiskar. Vårt protokoll möjliggör studier av molekylära mekanismer bakom hypoxi-inducerad cancermetastasering. I papper III visas, att systemisk administration av antiVEGF eller anti-VEGF-receptor (VEGFR)-2 neutraliserande antikroppar in en musmodell orsakar generell kärlregression. Bland alla undersökta vävnader påverkades endokrina körtlar, tarmslemhinna och uterus mest av VEGF eller VEGFR-2 blockad. Långvarig anti-VEGF behandling resulterade i en signifikant minskning av cirkulerande nivåer av det dominerande sköldkörtelhormonet, fritt tyroxin, men inte av trijodtyronin, vilket tyder på att kronisk anti-VEGF behandling försämrar sköldkörtelfunktionerna. Resultaten påvisar risken för biverkningar i friska vävnader av anti-VEGF behandling. I papper IV visar vi att störningar i dygnsrytm genom konstant exponering för ljus och genetisk manipulation av nyckelgener i zebrafisk ledde till nedsatt angiogenes under embryonal utveckling. En bmall-specifik morfolino hämmade angiogenes i zebrafisk utan att orsaka andra kärl-oberoende fenotyper. Omvänt, en period2 morfolino accelererade angiogeneskärltillväxt, vilket tyder på att Bmall och Period2 utövar motsatta effekter påkärlstillväxt. Dessa resultat ger mekanistisk kunskap om den roll som dygnsrytmen har i regleringen av angiogenes, och resultat kan rimligen utvidgas till andra typer av fysiologisk eller patologisk angiogenes. Sammanfattningsvis ger resultaten i denna avhandling ytterligare kunskap om angiogenetiska mekanismer och pekar på möjliga nya terapeutiska mål för behandling av olika angiogenes-beroende sjukdomar. 


\section{LIST OF PAPERS}

I. Ziquan Cao*, Lasse D Jensen*, Pegah Rouhi*, Kayoko Hosaka, Toste Länne, John F Steffensen, Erik Wahlberg, Yihai Cao. Hypoxia-induced retinopathy model in adult zebrafish. Nature Protocols. 2010 Dec;5(12):1903-10.

II. Pegah Rouhi*, Lasse D Jensen*, Ziquan Cao*, Kayoko Hosaka, Toste Länne, Eric Wahlberg, John Fleng Steffensen, Yihai Cao. Hypoxiainduced metastasis model in embryonic zebrafish. Nature Protocols. 2010 Dec;5(12):1911-8.

III. Yunlong Yang*, Yin Zhang*, Ziquan Cao*, Hong Ji, Xiaojuan Yang, Hideki Iwamoto, Eric Wahlberg, Toste Länne, Baocun Sun, Yihai Cao. Anti-VEGF- and anti-VEGF receptor-induced vascular alteration in mouse healthy tissues. Proceedings of National Academy Sciences of the United States of America. 2013 Jul 16;110(29):12018-23.

IV. Lasse Dahl Jensen, Ziquan Cao, Masaki Nakamura, Yunlong Yang, Lars Bräutigam, Patrik Andersson, Yin Zhang, Eric Wahlberg, Toste Länne, Kayoko Hosaka, Yihai Cao. Opposing Effects of Circadian Clock Genes Bmal1 and Period2 in Regulation of VEGF-Dependent Angiogenesis in Developing Zebrafish. Cell Reports. 2012 Aug 30;2(2):231-41.

*Equal Contribution 


\section{RELATED PUBLICATIONS}

I. Pegah Rouhi, Samantha Lin Chiou Lee, Ziquan Cao, Eva-Maria Hedlund, Lasse Dahl Jensen, Yihai Cao. Pathological angiogenesis facilitates tumor cell dissemination and metastasis. Cell Cycle. 2010 Mar $1 ; 9(5): 913-7$.

II. Lasse Dahl Jensen, Pegah Rouhi, Ziquan Cao, Toste Länne, Eric Wahlberg, Yihai Cao. Zebrafish models to study hypoxia-induced pathological angiogenesis in malignant and nonmalignant diseases. Birth Defects Research Part C Embryo Today. 2011 Jun;93(2):182-93.

III. Sharon Lim, Jennifer Honek, Yuan Xue, Takahiro Seki, Ziquan Cao, Patrik Andersson, Xiaojuan Yang, Kayoko Hosaka, Yihai Cao. Coldinduced activation of brown adipose tissue and adipose angiogenesis in mice. Nature Protocols. 2012 Mar 1;7(3):606-15.

IV. Mei Dong, Xiaoyan Yang, Sharon Lim, Ziquan Cao, Jennifer Honek, Huixia Lu, Cheng Zhang, Takahiro Seki, Kayoko Hosaka, Eric Wahlberg, Jianmin Yang, Lei Zhang, Toste Länne, Baocun Sun, Xuri Li, Yizhi Liu, Yun Zhang, Yihai Cao. Cold Exposure Promotes Atherosclerotic Plaque Growth and Instability via UCP1-Dependent Lipolysis. Cell Metabolism. 2013 Jul 2;18(1):118-29.

V. Xiaojuan Yang, Yin Zhang, Yunlong Yang, Sharon Lim, Ziquan Cao, Janusz Rak, Yihai Cao. Vascular endothelial growth factor-dependent spatiotemporal dual roles of placental growth factor in modulation of angiogenesis and tumor growth. Proceedings of National Academy Sciences of the United States of America. 2013 Aug 20;110(34):13932-7.

VI. Jian Wang, Ziquan Cao, Xing-Mei Zhang, Masaki Nakamura, Meili Sun, Johan Hartman, Robert A. Harris, Yuping Sun, Yihai Cao. Novel mechanism of macrophage-mediated metastasis revealed in a zebrafish model of tumor development. Cancer Research. 2015 Jan 15;75(2):30615. 


\section{ABBREVIATIONS}

AMD

Bmal1

CBF-1

ChIP

Clock

$\mathrm{CNV}$

Cry

CSL

cyc

DD

Dll

DR

ECM

EGF

EPC

EMA

FDA

FGF

GI

HBGF

Hes

Hey

HGF

HIF

hpf

HRE

$\mathrm{Ig}$

IL-6
Age-related macular degeneration

Brain and muscle ARNT-like-1

C-promoter binding factor 1

Chromatin Immunoprecipitation

Circadian locomotor output cycles kaput

Choroidal neovascularization

Cryptochrome

CBF-1, $\mathrm{Su}(\mathrm{H}), \mathrm{Lag}-1$

Cycel (gene)

Constant darkness

Delta like ligand

Diabetic retinopathy

Extracellular matrix

Epidermal growth factor

Endothelial progenitor cell

Europenan medicines agency

Food and drug administration

Fibroblast growth factor

Gastrointestinal

Heparin binding growth factor

Hairy enhancer of split

Hes-related protein

Hepatocyte growth factor

Hypoxia inducible factor

Hours postfertilization

Hypoxia response element

Immunoglobulin

Interleukin-6 


\begin{tabular}{|c|c|}
\hline IP & Intraperitoneally \\
\hline ISV & Intersegmental vessel \\
\hline KDR & Kinase inserted domain-containing receptor \\
\hline Lag-1 & Lin-12 and glp-1 \\
\hline LD & Switching between $12 \mathrm{~h}$ light and $12 \mathrm{~h}$ dark \\
\hline LL & Constant light \\
\hline MMP & Matrix metallo-proteinase \\
\hline NEC & Notch extracellular domain \\
\hline NICD & Notch intracellular domain \\
\hline NVG & Neovascular glaucoma \\
\hline $\mathrm{OM}$ & Optical microscope \\
\hline PC & Pericyte \\
\hline PDGF & Platelet-derived growth factor \\
\hline Per & Period \\
\hline PF-4 & Platelet factor-4 \\
\hline PlGF & Placenta growth factor \\
\hline qPCR & Quantitative real time-PCR \\
\hline RTK & Receptor tyrosine kinase \\
\hline RT-PCR & Reverse transcription-PCR \\
\hline SIV & Subintestinal veins \\
\hline SMC & Smooth muscle cell \\
\hline $\mathrm{Su}(\mathrm{H})$ & Suppressor of hairless \\
\hline sVEGFR & Soluble VEGFR \\
\hline TGF & Transforming growth factor \\
\hline TKI & Tyrosine kinase inhibitor \\
\hline $\mathrm{T} 3$ & Free triiodothyronine \\
\hline $\mathrm{T} 4$ & Free thyroxine \\
\hline VEGF & Vascular endothelial growth factor \\
\hline VEGFR & Vascular endothelial growth factor receptor \\
\hline VPF & Vascular permeability factor \\
\hline VPR & VEGF-related protein \\
\hline
\end{tabular}




\section{INTRODUCTION}

\section{ANGIOGENESIS}

\subsection{Biological Functions of Vessels}

During the embryonic development process in humans, the vasculature is the first developed functional system. Its main function is to deliver nutrients, oxygen and hormones secreted by the endocrine organs to tissues in the whole body, and to transport carbon dioxide, urea and other metabolites from the tissues and organs to eliminate from the body. It is well-established that the vasculature is an indispensable organ in maintaining normal metabolism and other physiological activities in the body. The vascular system includes cardiovascular system and lymphatic vascular system, with blood vessels sub-divided into arteries, veins and capillaries based on their structure and function.

\subsection{Concept of Newly Formed Blood Vessels}

The growth process of the vascular system is categorized into vasculogenesis, arteriogenesis and angiogenesis ${ }^{1,2}$. In the embryonic vascular development stage and after birth, bone marrow-derived endothelial progenitor cells (EPCs) migrate to specific sites, where they differentiate into mature endothelial cells, and gradually form the vascular lumen and, eventually, the vascular network. This process is called vasculogenesis ${ }^{3}$. Arteriogenesis is the process in which blood flow is redirected into the collateral arteriole after the main artery is blocked, which causes cell proliferation and vascular remodeling, thereby forming enlarged functional arteries from smaller vessels ${ }^{4,5}$. Angiogenesis refers to the neovascularization process from pre-existing vascular endothelial cells in the capillaries through continuous proliferation and migration ${ }^{4,6-10}$.

\subsection{Background of Angiogenesis}

Dr. John Hunter first proposed the concept of "angiogenesis" in 1787 to describe the growth of new vessels during development in antlers of the Fallow Deer ${ }^{6}$. However, over the following hundred years, angiogenesis is rarely mentioned in 
exploration of related diseases, and almost all focus are on anatomical studies. This was the case until the mid-19th century when Thiersch demonstrated that new vessels originated from preexisting capillaries ${ }^{11}$. Later, it was also found that tumor tissues, relative to normal tissues, were rich in blood vessels, which was generally thought to be associated with inflammation. The relationship between the growth of blood vessels and the growth of tumors was not considered.

It was recognized early on that cells in humans and other mammals cannot live without blood vessels. Moreover, the responsibility of blood vessels for transportation and exchange of nutrients, gases, hormones and cells among the tissues and organs was given great importance. That blood vessels, or specifically the endothelium, themselves produce various active substances, which makes it a vital endocrine organ in the body was discovered in $1960 \mathrm{~s}^{12}$. Other important discoveries includes the findings that the distance limit between the cells and the vessels of $100-200 \mu \mathrm{m}$ in diameter is crucial to ensure diffusion of the oxygen from the red blood cells in the blood vessels into the cells. Accordingly, early developing primary solid tumors during the dormant period can maintain their growth only through penetration of the peripheral interstitial fluid ${ }^{11,13,14}$. In 1971, Dr. Judah Folkman proposed for the first time that the growth and metastasis of solid tumors were dependent on angiogenesis ${ }^{8}$.

\subsection{The Angiogenic Switch}

Under normal conditions, angiogenic activators that can stimulate angiogenesis and angiogenic inhibitors that prevent angiogenic processes are responsible for regulating angiogenesis. The activation of angiogenesis is called the "angiogenic switch". When activators of angiogenesis become dominant, the angiogenic switch is turned on. Angiogenic activators includes growth factors such as vascular endothelial growth factor $(\mathrm{VEGF})^{15}$, platelet-derived growth factor $(\mathrm{PDGF})^{16}$, fibroblast growth factor $(\mathrm{FGF})^{17,18}$, and transforming growth factor (TGF). Angiogenesis inhibitors include angiostatin, thrombospondin-1, platelet factor-4 (PF-4), matrix metalloproteinase (MMP) inhibitors. These factors act directly on vascular endothelial cells leading to proliferation and migration of vascular endothelial cells.

\subsection{Process of Angiogenesis}


Growth factors, in particular VEGF, promote angiogenesis. VEGF is a heparin binding growth factor that specifically acts upon endothelial cells. VEGF, among several angiogenic factors, can directly stimulate endothelial cells to make them migrate and proliferate. Vascular endothelial cells are a layer of tabular epithelial cells that form the inner walls of blood vessels. Under normal conditions, these cells display a long interval cycle of division and proliferation, and they are some of the most long-lived human cells. Under physiological and pathological angiogenesis, the activated cells can secrete proteases required for angiogenesis, and these proteases like MMPs are capable of degrading basement membrane and extracellular matrix to dissociate endothelial cells. Meanwhile, the endothelial cells are able to proliferate and migrate along the concentration gradient of growth factors (e.g., VEGF and FGF), whereby they become tip cells and stalk cells.

Tip cells lead the sprouting vessels, by extending filopodias along the gradient of angiogenic stimuli as a directional guidance, and induce bud growth ${ }^{19,20}$. Adjacent stalk cells, that generate the trunks of the vessels, proliferate to form capillary-like lumens ${ }^{20,21}$, and are covered by mural cells, pericytes (PCs) and vascular smooth muscle cells (SMCs). Ultimately, vascular networks are established and the vasculature becomes mature ${ }^{22}$.

In healthy humans, wound healing, the menstrual cycle and fetal growth and development are accompanied by physiological angiogenesis ${ }^{23}$. Pathological angiogenesis takes place during tumor growth, rheumatoid arthritis, retinopathy, ischemic cardiocerebral vascular diseases, obesity, diabetes, and other various diseases ${ }^{24-30}$. A sufficient grasp of the knowledge about newly formed vessels is vital to the study how angiogenesis is implicated in all these diseases (see Figure $1)$.

\subsection{ANGIOGENESIS IN DISEASES}

Vascular homeostasis is an important foundation of the vital functions in the body and it plays an important role in maintaining the normal physiological state. The key for maintaining vascular homeostasis is a balanced and functioning vascular network. Normally, when the networks have been established, the endothelial cells remain in a quiescent state and no new vessels develop. 


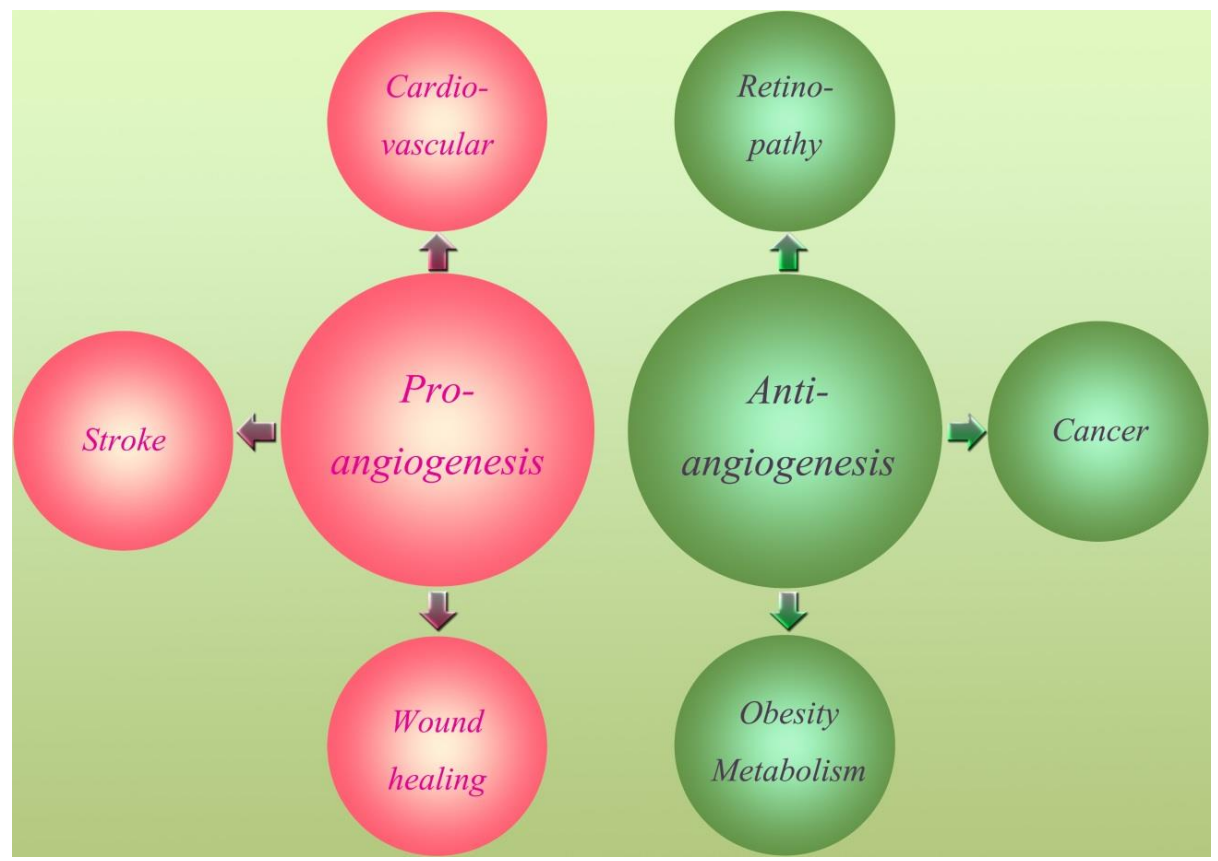

Figure 1. Angiogenesis in disease.

Nevertheless, under the influence of physical, chemical, biological and other internal and external environmental changes, the balanced system controlling blood vessel growth might be disrupted, and the development of new vessels is initiated, and the resulting process and final vessel network might even be chaotic. Thereby, angiogenesis occurs and the function and structure of the blood vessels change, which is a consequence or a prerequisite for pathological course of disease. Targeting angiogenesis, therefore, is a potential target for the treatment of cancer. Likewise there are ischemic diseases with a main feature of lack of delivery of oxygen and nutrients to tissue. The angiogenic process is then impaired and the balanced system is set at a very low level of new vessel formation in spite of the great need ${ }^{31}$. Then, promoting angiogenesis may be a beneficial therapeutic strategy.

\subsubsection{Angiogenesis in Retinopathy}

According to many studies pathological angiogenesis is closely related to VEGF expression, which for example may lead to development and occurrence of a variety of eye diseases ${ }^{32-36}$, including diabetic retinopathy (DR), age-related macular degeneration (AMD), neovascular glaucoma (NVG), and retinopathy of 
prematurity. These diseases induced by abnormal angiogenesis are all related to over-expression of VEGF. The majority of new vessels formed in these diseases are not structurally completed, and the resulting hemorrhage, fibrosis and fluid secretions often lead to blindness ${ }^{37}$.

DR, one of the most common and serious complications of diabetic microangiopathy has become the primary cause of blindness in many countries $^{38,39}$. The retinal neovascularization eventually results in retinal and vitreous hemorrhage leading to tractional detachment of retina and consequently blindness.

The primary lesion of AMD is choroidal neovascularization (CNV), in which VEGF plays an important role. During CNV, choroid blood circulation changes and anaerobic conditions ${ }^{36}$ occur resulting in HIF-1 $\alpha$ (hypoxia inducible factor$1 \alpha)$ and increased VEGF signaling ${ }^{40}$. Therefore, targeting these pathways may be a potential way to control $\mathrm{CNV}$ formation.

NVG is an angiogenesis-induced eye disease that results in angiotelectasis in the iris and anterior chamber. The accompanying intraocular hypertension often causes blindness as a result of this. Angiogenesis in the iris and anterior chamber angle is induced by ischemic conditions resulting in the release of VEGF due to hypoxia. Studies ${ }^{41,4241,4241,4241,4241,4241,4241,4240,4139,4038,3937,3837,3837,38}$ have indicated that VEGF levels in the aqueous fluid and vitreous body significantly increases in NVG-related eye diseases.

Although risk factors for many eye diseases not are clear, many drugs for treatment of angiogenesis-related eye diseases that are available that are based on the mechanism of inhibition of VEGF. Bevacizumab (with the trade name Avastin), an anti-VEGF antibody and the first FDA (U.S. Food and drug administration)-approved anti-angiogenic and anti-cancer agent in 2004, can inhibit proliferation of endothelial cells and reduce vascular permeability resulting in the blockage of neovascularization ${ }^{43}$. With in-depth understanding of bevacizumab through a large number of clinical trials, it has been applied increasingly in many different diseases ${ }^{44}$, also outside the eye. Additionally, it has demonstrated very significant clinical efficacy in the treatment of vascular proliferation-induced eye diseases ${ }^{45-51}$. Results from clinical trials have certified 
that intravitreal injection of bevacizumab is safe and effective treatment method, and that this agent can stabilize or improve the visual acuity, and effectively control the intraocular pressure of patients. Furthermore, few inflammatory or other complications have been recorded ${ }^{52}$.

In June 2006, the FDA approved ranibizumab (trade name Lucentis) for treatment of exudative AMD. It nonspecifically binds to activated VEGF thus blocking the interaction between VEGF and its receptor, thereby decreasing neovascularization ${ }^{53}$. The European medicines agency (EMA) also approved ranibizumab for treatment of visual impairments induced by wet-AMD, diabetic macular edema, macular edema secondary to retinal vein occlusion and myopic choroidal neovascularization. In a recent phase III, double-blind, randomized, positively controlled clinical trial, it was shown that an intravitreal injection of ranibizumab was more effective than other therapies in treating myopic $\mathrm{CNV}^{54}$.

\subsubsection{Angiogenesis in Cancer}

Currently, many diseases are known to be related to angiogenesis and cancer is the most widely studied of these diseases. Cancer, also known as neoplasmic disease, is divided into malignant and non-malignant and it is one of the most common diseases in humans that are caused by uncontrolled cell growth and proliferation. Through many studies, the biological hallmarks of cancer have been revealed to include; persistent proliferation signals, insensitivity to growth inhibition, apoptosis resistance, immortalization, induction of angiogenesis, activating invasion and metastasis, reconstructing metabolic system of energy and avoiding destruction by the immune system ${ }^{55}$. Methods to treat tumors have been studied for a long time. As part of these studies, angiogenesis mediated by tumor-derived growth factors has become one of the most important targets for tumor therapy research.

Because endothelial cells in newly formed vessels proliferate rapidly, they are very sensitive to chemotherapeutic drugs. However, specific agents that target the angiogenic process have also started to be developed. So far, anti-angiogenic therapy is recognized as very promising anti-cancer therapy strategy. Work in this field stems from Dr. Judah Folkman's theory, prostulated more than 40 years ago, where he proposed that the growth and metastasis of all solid tumors was 
dependent on blood vessel growth, and thus inhibition of angiogenesis could be an effective means of cancer treatment ${ }^{8}$.

Tumors do not generally grow beyond $2 \mathrm{~mm}^{3}$ without oxygen and nutrients provided by blood vessels. Therefore, angiogenesis is the key factor to support continuing tumor growth. Growth factors secreted by the tumor cells promote growth and development of new blood vessels. Tumor neovascularization, however, is completely different from vessels in the host tissue. Morphologically tumor vessels are irregular, torturous and heterogeneous, leaky and with an abnormal basement membrane ${ }^{56}$. Therefore studies of angiogenesis and the mechanism behind it are fundamental and crucial for treatment of cancer.

\subsubsection{Angiogenesis in Cardiovascular Disease}

Research of the mechanism of neovascularization can not only lead to the possibility of inhibiting the growth of blood vessels to treat different diseases, but also suggest therapeutic targets for ischemia-related diseases, such as coronary heart disease, peripheral arterial disease and cerebral thrombosis.

The reduction of perfusion or inhibition in bloods ability to deliver and remove molecules to organs or tissues results in ischemia. Ischemia is generally caused by local hemodynamic disorders in larger vessels, and sometimes by local manifestations of this anematosis. At present, ischemic heart disease is a leading disease worldwide in causing morbidity and mortality. Coronary hypoperfusioninduced myocardial ischemia and the resulting hypoxia in the heart muscle is the main problem in advanced ischemic heart disease causing both myocardial infarction and long term also congestive heart failure. Strategies, that may be able to complement interventional treatment for such diseases, can involve the infusion of proangiogenic factors into the myocardium or ischemic area resulting in stimulation of the formation and maturation of new vessels to enhance blood perfusion to the ischemic area. Animal experiments have demonstrated that proangiogenic factors, such as VEGF, can be administered to the ischemic myocardium using a carrier protein or gene via cardiac catheterization and this minimally invasive treatment method may cause increased angiogenesis ${ }^{57-60}$. Phase I clinical trials using infused growth factors has been conducted in patients with coronary heart disease ${ }^{61,62}$. In one of these trials, growth factors were injected into the ischemic area of myocardium, and it was observed that the score 
assessing the function of collateral vessels increased. More importantly, the results also suggested that proangiogenic gene therapy through intramyocardial injection was safe and tolerable. In the latter study it was impossible to objectively assess how efficacious the experimental treatment due to lack of control group. Further studies and clinical trials are needed to confirm the effectiveness and safety of proangiogenic therapy in cardiovascular diseases. In addition, VEGF not only promote neovascularization of ischemic myocardium, but also enhance blood perfusion of normal myocardium without pathological alterations. Therefore, injection of VEGF into non-ischemic myocardium may even be an effective prevention strategy for early ischemic heart disease ${ }^{63}$.

\subsubsection{Circadian Rhythm and Related Diseases}

The circadian clock is a widespread vital phenomenon influencing living things, including prokaryotic and single-celled organisms, other mammals and humans. The basis for the circadian clock is the expression of circadian clock genes. The day-night rhythm, the most common circadian rhythm in nature, refers to an adaptive response of living beings to the light-dark cycle in their environment, allowing the body behavior and physiological activity to act continuously throughout a 24-hour cycle ${ }^{64,65}$. Many physiological behaviors are regulated by day-night rhythms in human and other mammals including feeding/fasting and activity/resting 66,67 .

The genes involved in circadian rhythms in organisms include circadian locomotor output cycles kaput (Clock), brain and muscle ARNT-like-1 (Bmall), period $(P e r)$, cryptochrome $(C r y)$, cycle $(C y c)$ amongst others ${ }^{68-71}$, and these genes participate in many physiological and biochemical activities in the body, including cell growth and differentiation ${ }^{72}$. A series of physiological processes in vivo are closely associated with regulation of the expression of circadian clock genes, for instance blood pressure regulation, respiration rate, heart rate, metabolism and hormone secretion ${ }^{66,73-76}$. Studies have confirmed that disturbed day-night/light-dark rhythm might induce many diseases including cancer, diabetes, myocardial infarction, obesity, stroke, depressive disorder and rheumatoid arthritis ${ }^{77-83}$. Additionally, these diseases are often accompanied by pathological changes in vascular structures and vascular function ${ }^{27,84-87}$, including the occurrence of angiogenesis. 
Circadian clock genes (e.g., Bmall) may regulate angiogenesis by the influence of the circadian rhythm. As such, VEGF expression levels change with the circadian rhythm. Investigating circadian clock genes in relation with angiogenesis, therefore, would enable better understanding of the mechanism of pathological angiogenesis in diseases caused by disrupted circadian rhythm.

\section{VEGF FAMILY OF PROTEINS}

\subsection{Background of VEGF}

As mentioned before is the vasculature pivotal in development, maturation and homeostatic maintenance of vertebrates. The establishment of vessels depend on growth of endothelial cells that is stimulated by various growth factors (e.g., VEGF, PDGF, FGF, epidermal growth factor (EGF), TGF, etc. ${ }^{88}$. Growth factors are polypeptides that can bind to specific receptors on the plasma membrane to switch on a fast protein cascade which leads to DNA replication and cell division.

These growth factors activate quiescent endothelial cells leading to the process of physiological or pathological neovascularization. Among them, the VEGF family of multifunctional cytokines directly or indirectly regulates vasculogenesis, angiogenesis and lymphangiogenesis.

In 1983, Senger et al identified a factor that efficiently increased the vascular permeability in animal tumor models, and they called it vascular permeability factor (VPF) ${ }^{89}$. In 1989, Ferrara and his colleagues isolated and purified a factor that was widely distributed in the tissues of human and mammals that specifically could promote vascular endothelial cell mitosis, which they named VEGF $^{90}$. In the subsequent studies VPF and VEGF were confirmed to be the same protein encoded by the same gene consisting of two single-strands. VEGF was shown to have a lower synthesis levels in healthy tissues of adult humans and animals, but higher expression in the embryo and during physiological or pathological neovascularization.

VEGF has an extremely strong ability to induce vascular permeability ${ }^{89}$. Generally, increased vascular permeability is necessary for physiological 
processes, but it can often lead to negative consequences during pathological processes. For example, increased vascular permeability in a tumor might aid metastasis formation from a primary tumor.

The members of the VEGF family are dimeric glycoproteins with a molecular weight of between 34 to $45 \mathrm{kDa}$. This family can be divided into five isoforms; VEGF-A, VEGF-B, VEGF-C, VEGF-D and placenta growth factor (PIGF) in humans and other mammals. In addition, a protein related to the structure of VEGF was noted in the Orf virus, which came to be known as VEGF-E ${ }^{91}$, Another group of proteins called VEGF-Fs were found in snake venom isolated from the Horned viper and Russell's viper ${ }^{92,93}$. Each isoform of VEGF forms dimers and bind to their receptor causing dimerization of the receptor which consists of any combination of three VEGF receptors (VEGFRs) as shown in Figure $2^{94}$. Although there are various combinations for each of the ligands and receptors, each combination would promote formation and development of vessels, lymph vessels and other vasculatures.

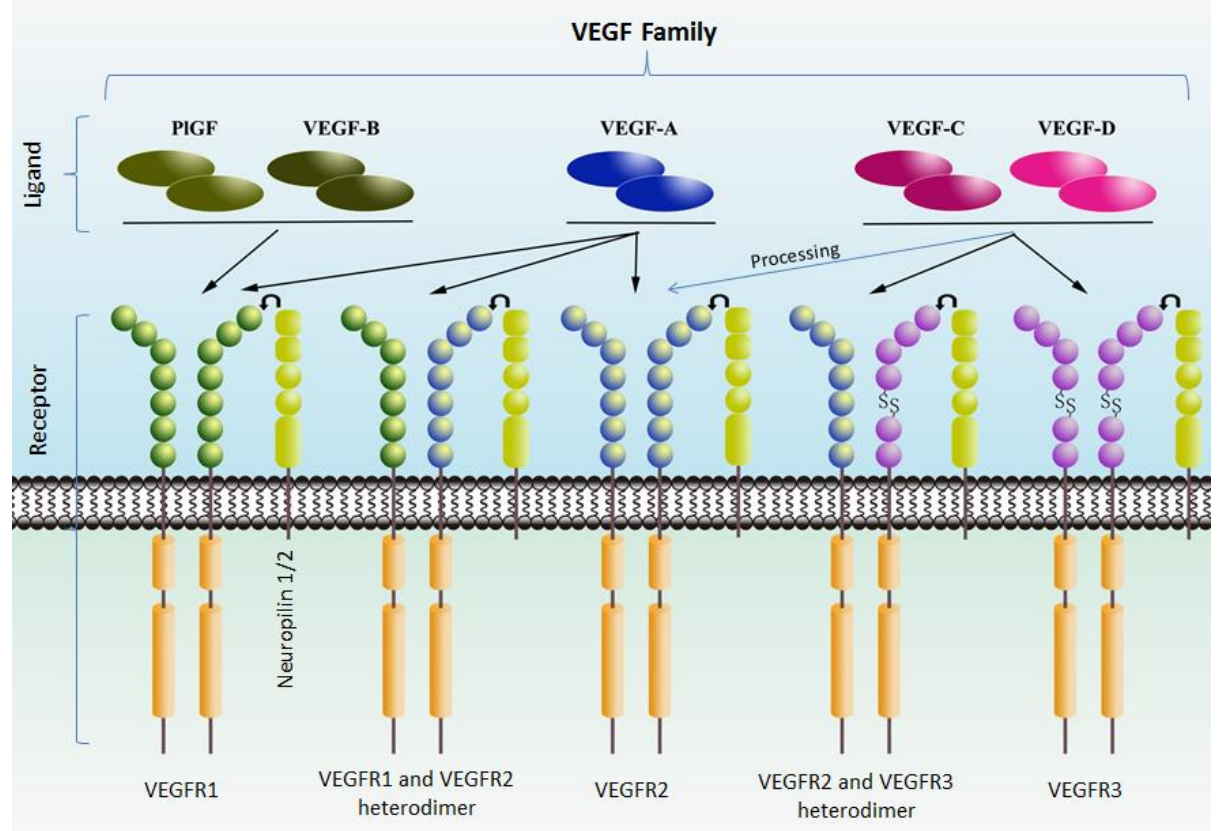

Figure 2. The VEGF family and VEGF receptors.

\subsection{VEGF ligands and Functions}

\subsubsection{VEGF-A}


VEGF-A, usually called VEGF, is a highly specific vascular endothelial cell mitogen. Furthermore, it is also the strongest pro-angiogenic factor in VEGF family and it is expressed in every organ and tissue in the body ${ }^{95,96}$. Hence, it is one of the most studied growth factors. The VEGF-A gene, containing eight exons and seven introns in the coding region, generates various isoforms after transcription, mRNA splicing variation and other steps that all have different biological properties. Among them, the isoforms of human VEGF-A are labeled as VEGF-A121, VEGF-A145, VEGF-A165, VEGF-A189, VEGF-A206 ${ }^{97,98}$. The functional difference among these isoforms is reflected by different binding activity between them and the heparin in the extracellular matrix. VEGF-A165 is the most common isoform that shows a major effect ${ }^{99,100}$.

\subsubsection{VEGF-B}

VEGF-B has a relatively high expression in myocardium and skeletal muscle ${ }^{101}$. VEGF-B has two different transcripts, VEGF-B167 and VEGF-B186. VEGFB186 is secreted from the cytoplasm into the extracellular matrix, while VEGFB167 is secreted only under the stimulation by heparin. Furthermore, VEGF-B binds to VEGF-A to form a heterodimer.

\subsubsection{VEGF-C}

VEGF-C with the molecular weight of $46.9 \mathrm{kDa}$ was purified first by Joukov et al. in $1996^{102}$. Lee et al. also isolated the corresponding gene, and the protein encoded by this gene was named VEGF-related protein (VRP) ${ }^{103}$. VEGF-C is a mitogen for lymphatic endothelial cells, and is involved in the development of lymphatic system ${ }^{104,105}$, and can promote lymphangiogenesis and induce lymphatic hyperplasia ${ }^{106,107}$. Its role in mediating lymphangiogenesis is caused by its ability to effectively regulate activation, proliferation and migration of lymphatic endothelial cells. As lymph node metastasis may occur by invasion of tumor cells into the lymphatic vessels, VEGF-C, is closely associated with the tumor metastasis ${ }^{102}$. Clinical data has demonstrated a correlation between VEGF$\mathrm{C}$ levels in the tumor and metastasis process ${ }^{108,109}$. Additionally, VEGF-C may induce microvascular endothelial cell proliferation to some extent due to its ability to bind to VEGFR-2 ${ }^{110}$.

\subsubsection{VEGF-D}


VEGF-D is similar to VEGF-C in structure. VEGF-D mRNA can be detected in the majority of human tissues, and is relatively abundant in the myocardium, lung, skeletal muscle, colon, and small intestine of adults ${ }^{111}$. It not only induces lymphangiogenesis in tumors, but also causes diffusion of the tumor cells towards the regional lymph nodes through its expression in the tumor cells ${ }^{112}$.

\subsubsection{VEGF-E}

VEGF-E with the molecular weight of $20 \mathrm{kDa}$ is absent in humans and other mammals. It is, however, found to have the similar biological effects as VEGFA165. At the protein level it demonstrates a $\sim 25 \%$ sequence homology with VEGF-A165 at the amino acid level, though it lacks the basic domain and heparin binding domain ${ }^{113}$.

\subsubsection{PIGF}

PlGF is a secreted glycoprotein, and is a dimer that first was isolated and purified from cDNA in human placenta by Iyer et $\mathrm{al}^{114}$. There are four family members in this group, PlGF-1, PlGF-2, PlGF-3 and PlGF-4 based on the different mRNA splicing variants. PIGF is only be expressed in various kinds of tumors, but also in placenta in large amounts ${ }^{115,116}$, which lasts the entire pregnancy, hence its name. P1GF can also bind to VEGF-A to form a heterodimer ${ }^{117}$.

\subsection{VEGF Receptor and Signaling}

The activation of the classical VEGF biological signal transduction pathways require the specific binding of VEGF ligands and three types of transmembrane receptor tyrosine kinases (RTKs) in the cell membrane (VEGFR-1 (Flt-1), VEGFR-2 (KDR/FlK-1) and VEGFR-3 (Flt-4)) as. Among them VEGFR-2 is expressed in all endothelial cells and is the most important receptor mediating vasculogenesis. Human VEGFR-2 is called kinase inserted domain-containing receptor (KDR), while the murine receptor is called fetal liver kinase-1 (Flk-1).

\subsubsection{Structure of VEGF Receptors}

VEGFRs are members of the RTK superfamily and made up of one extracellular domain, one transmembrane domain and one intracellular domain ${ }^{118,119}$. Among them, the extracellular domain is composed of seven immunoglobulin (Ig)-like folds ${ }^{91}$. The intracellular domain contains two tyrosine kinase domains with a C- 
terminal tail at the end, and various cell signals are mediated from intracellular domain to downstream of signaling cascades ${ }^{120}$.

The activation of VEGFRs is controlled by its ligand. After the extracellular VEGFs dimer binds to the corresponding VEGFRs, the monomeric vEGFRs dimerize. According to the differences of VEGFs dimer structures and the specific binding capacity to VEGFRs, the dimerized VEGFRs will form into a homodimer or a heterodimer ${ }^{121}$. Dimerized tyrosine kinases are activated and induce phosphorylation of its own tyrosine residues resulting in the phosphorylation and activation of other downstream proteins and pathways including the activation of a series of the second messengers.

\subsubsection{VEGF Receptor-1}

Though VEGFR-1 was the first discovered RTK receptor of VEGF, its function is controversial due to its many roles. It regulates different signal pathways in various cell types and stages of the cell cycle. VEGF-A, VEGF-B and PIGF are all ligands of VEGFR-1. Due to various splicing variants, VEGFR-1 may also produce soluble VEGFR-1 (sVEGFR-1). VEGFR-1 and soluble VEGFR-1 can both bind to VEGF-A, with high binding affinity but low response level compared to VEGFR-2 ${ }^{122,123}$. Park et al. thought that VEGFR-1 is a decoy receptor that creates no active signal transduction for cell mitosis working as negative regulator of VEGF ${ }^{124}$. The other possibility is that VEGFR-1 could block angiogenic cell signals through competing with VEGFR-2 for binding of VEGF-A. It may then lead to less VEGF binding to VEGFR-2. VEGF-B and PIGF can only bind to VEGFR-1 and not to VEGFR-2 and VEGFR-3. Ligand binded-VEGFR-1 induces phosphorylation in the intracellular domain resulting in the recruitment of VEGFR-1 expressing cells such as monocytes as well as secretion of some factors, such as Hepatocyte growth factor (HGF) and Interleukin-6 (IL-6).

\subsubsection{VEGF Receptor-2}

With its highly specific expression in endothelial cells ${ }^{125}$, and as being the main functional receptor of VEGF-A, VEGFR-2 plays a leading role in VEGF mediated signaling and vascular endothelial growth. VEGF-A bound to VEGFR2 activates a series of intracellular downstream signal molecules related to mitogenic, chemotactic and anti-apoptotic effects. Under stimulation of VEGF-A, 
tyrosine residues at different locations on VEGFR-2 can bind to multiple proteins, which mediate signals and induce phosphorylation and initiation of a signal cascade reaction, regulating the biological characteristics of endothelial cells through different activation pathways (e.g., endothelial cell migration, cell proliferation, actin remodeling and anti-apoptosis). Eventually, VEGF-AVEGFR-2 signaling promotes angiogenesis. Coupled with this effect on angiogenesis, VEGFR-2 can also participate in VEGF-A-mediated increases in vascular permeability ${ }^{126-128}$.

VEGF-C and VEGF-D also bind to VEGFR-2. Even though VEGF-E is absent in humans and other mammals, VEGF-E can bind specifically to VEGFR-2 and mediate the biological effects similar to VEGF-A. For example, in the rat tumor and cornea models, angiogenesis mediated by VEGF-E can be detected and new vessels are similar to those produced by VEGF-A mediated signaling in their form, structure and function.

\subsubsection{VEGF Receptor-3}

VEGFR-3 is mainly expressed on endothelial cells of lymphatic vessels ${ }^{104,129}$ and is the specific receptor of VEGF-C and VEGF-D. VEGF-C and VEGF-D bound VEGFR-3 activate downstream signaling pathways and induce lymphatic endothelial cell proliferation, migration and formation of the lymphoid sinus ${ }^{130}$, thus causing lymphatic hyperplasia. VEGFR-3 also plays a vital role in cardiovascular formation during embryonic development. During late embryonic development VEGFR-3 is expressed mainly in lymphatic vessels and to a small degree in blood vessels. Only one layer of endothelial cells with an irregular basement membrane forms the lymphatic capillaries ${ }^{131,132}$. Malignant tumor cells can therefore enter the lymphatic vessels easily, whereas tumor cells need to penetrate a well-aligned basement membrane to intravasate blood vessels ${ }^{133}$.

\section{CELL SIGNALING IN ANGIOGENESIS}

\subsection{Hypoxia in Angiogenesis}

Pathological angiogenesis is often accompanied by tissue hypoxia ${ }^{134}$. Hypoxia causes a series of alterations of processes in tissues and cells to allow adaptation to low oxygen environments. For example, under hypoxic conditions angiogenesis, cell proliferation, cell survival, ion metabolism and carbohydrate 
metabolism change, and genes that are involved in the hypoxic response are induced $^{135}$. Among them, hypoxia-inducible factor-1 (HIF-1) is a vital transcriptional regulatory factor in such adaptations ${ }^{136}$. HIF-1 transcribes and activates the expression of a series of genes under hypoxia, and especially upregulation of $\mathrm{VEGF}^{137}$. Since hypoxia can induce functional alterations in the body, it is recognized as one of the most prominent processes that cause diseases even leading to death. This has led to a great number of studies evaluating angiogenesis under hypoxic conditions.

\subsection{HIF/VEGF signaling pathway}

\subsubsection{Structure and Functions of HIF}

HIF is a transcription factor that is upregulated when tissue ischemia and hypoxia cause a lowered oxygen concentration in the cells. HIF induces certain regulatory effects. The HIF family includes HIF-1, HIF-2 and HIF-3 ${ }^{138}$. Among them, HIF1 is a heterodimer transcription factor comprised of protein subunits $\alpha$ and $\beta$. They were first discovered in hypoxia-induced cell extracts by Semenza et al. in $1992^{139}$. HIF-1 $\alpha$ is the main regulator of the hypoxic response and is highly sensitive to the oxygen concentration. It only consists of an oxygen regulation subunit 140,141 therefore it determines the activity of HIF-1 depending on oxygen concentration $^{142}$. HIF-1 $\beta$ shows constitutive expression in cells and is not affected by the oxygen concentration ${ }^{143,144}$. When HIF- $1 \alpha$ forms a dimer with HIF-1 $\beta$, HIF-1 is activated.

\subsubsection{HIF Pathway}

Under normoxic conditions, HIF- $1 \alpha$ is degraded by proteasomal degradation ${ }^{145}$. Under hypoxic conditions owing to hydroxylation reaction inhibition, it cannot be degraded, leading to accumulation in cells. Then HIF-1 $\alpha$ enters into the nucleus and binds to HIF-1 $\beta$ to form heterodimers. Following that process, the heterodimer binds specifically to the hypoxia response element (HRE) on the VEGF promoter or enhancer. This increases the stability of VEGF mRNA and also promotes up-regulation and transcription of VEGF and its receptors' expression. Hypoxia is the main stimulus of $\mathrm{VEGF}^{146}$, which binds specifically to VEGFR on vascular endothelial cells to activate a series of transduction pathways in response to ischemia to induce neovascularization ${ }^{147}$. 


\subsection{Notch signaling pathway}

\subsubsection{Background of Notch}

The Notch signaling pathway plays different roles in various processes during development. This signaling is essential in ensuring normal embryonic development and maintaining steady state of tissues and stem cells ${ }^{148}$. In 1919, the Notch gene was first discovered in fruit flies and it is an evolutionarily highly conserved family of transmembrane receptors ${ }^{149}$, The Notch signaling pathway comprises of Notch ligand, Notch receptor and CSL (C-promoter binding factor 1 (CBF-1), suppressor of hairless $(\mathrm{Su}(\mathrm{H}))$, lin-12 and glp-1 (Lag-1)) DNAbinding protein ${ }^{150}$. Upon binding of the Notch ligand to its receptor, Notch signal transduction is activated and this regulates cell differentiation and histogenesis. Mutation of Notch or its ligand may lead to developmental defects in the heart, skeleton, hematopoiesis- and nervous system. It is also shown that Notch is of significance in various processes of vasculogenesis and angiogenesis ${ }^{151,152}$.

\section{Structure and Functions of Notch}

There are four different Notch receptors in vertebrates, i.e., Notch1, Notch2, Notch3 and Notch4. Structurally the Notch receptor consists of Notch extracellular domain (NEC), a transmembrane domain and the Notch intracellular domain (NICD). The main function of the NEC is to bind to ligands on adjacent cells to activate the Notch signaling pathway. The Notch ligand is a single-pass transmembrane protein expressed on the cell surface and there are five types in mammals: jagged1, jagged2, Delta like ligand 1 (Dl11), Delta like ligand 2 (Dll3) and Delta like ligand 4 (Dll4). Notch signal transduction requires several steps during the activation process. In detail, after the Notch ligand bind to the receptor, a site on the membrane of Notch receptor is cut by $\gamma$-secretase to release NICD. Then, NICD enters the cytoplasm and is transferred into the cell nucleus. Finally, NICD interacts with CSL and converts CSL to a transcriptional activator ${ }^{153,154}$, the downstream transcriptional factors, such as hairy enhancer of split (Hes) and Hes-related protein (Hey) families, are targets of Notch/CSL-dependent signaling ${ }^{155-157}$ and further regulate the expression level of downstream molecules to regulate expression of cell differentiation-related genes ${ }^{158,159}$.

\subsubsection{DII4: The Ligand of Notch}

Dl14 is one of the ligands of the Notch signal family in mammals ${ }^{160}$. Dl14, much like VEGF, is the gene related to vascular growth and development. It is 
expressed during physiological and pathogenical angiogenesis and plays a key role in vasculogenesis and angiogenesis as well as in maintaining vascular homeostasis $^{161}$. Dll4, therefore, has potential clinical applications ${ }^{162,163}$. In particular Dl14, which is not only expressed restrictively in the aortic endothelial cells during embryonic development ${ }^{151,160,164}$, but also the only ligand expressed in tip cells during vascular budding ${ }^{165}$. The Dl14/Notch signal plays a key role in the process of differentiation from tip cell to stalk cells in endothelial cells.

D114 belongs to a group of hypoxia regulatory genes ${ }^{166}$. In the classical HIF-1 $\alpha$ signaling pathway, after Dl14 ligands and receptor binding, NICD and HIF-1 $\alpha$ interact with each other and during hypoxia HIF-1 $\alpha$ is recruited as a Notch response promoter to activate Notch signaling. Therefore hypoxia promotes and stabilizes the activity of $\mathrm{NICD}^{167}$, and strengthens the final signal expression. Many details, however, of the Notch signaling pathway and the connections between different cell signaling pathways are still undefined.

Experimental studies have demonstrated that VEGF can induce the tip cell phenotype ${ }^{168}$, and the expression of Dll4 in endothelial cells regulates the budding and branching of new vessels. Further research confirmed that angiogenesis is under the control of VEGF and Dll4/Notch signaling pathways' synergistic effect. VEGF interacts with Dll4/Notch signaling pathway, that is, on one hand, VEFG induces Notch signal, on the other hand, Notch signal can act reversely on VEGF, regulating the strength of the VEGF signal pathway to allow angiogenesis to proceed in an orderly way ${ }^{169}$.

Vasculogenesis is influenced by, and strictly regulated by endogenic vessel growth regulator. There are two types of vasculogenesis regulators: angiogenic stimulators and angiogenic inhibitors. The balance between these two types of factors and their biological functions influence the results of physiological or pathological angiogenesis.

\section{ANGIOGENIC EFFECTORS}

Angiogenesis is the result of co-regulation of VEGF and many other growth factors and cytokines ${ }^{170}$. For instance, PDGF is an important mitogenic factor, 
and is a multi-functional protein synthesized and excreted by platelets, endothelial cells, vascular smooth muscle cells and fibroblasts amongst others, and is able to stimulate the division and proliferation of various cell types.

\subsection{Other angiogenic stimulators}

\subsubsection{PDGF Family}

PDGFs is a dimer composed of two highly isogenous chains of -A, -B, -C, or -D through disulfide bonds. There are five isoforms: PDGF-AA, PDGF-BB, PDGFCC, PDGF-DD, and the heterodimer PDGF-AB ${ }^{171}$. PDGFs must bind to the corresponding PDGFRs on the cell membrane in order to have a biological effect. When PDGFs bind to PDGFRs, dimerization occurs and produces three receptor isoforms, PDGFR- $\alpha \alpha$, PDGFR- $\beta \beta$ or PDGFR- $\alpha \beta$. PDGF-AA binds only to PDGFR- $\alpha \alpha$, while PDGF-CC binds to both PDGFR- $\alpha \alpha$ and PDGFR- $\alpha \beta$. PDGFDD binds only to PDGFR- $\beta \beta$, while PDGF-BB binds to all three receptor isoforms, PDGFR- $\alpha \alpha$, PDGFR- $\beta \beta$ and PDGFR- $\alpha \beta$. There are evidence that PDGFs and the corresponding receptors play a role in regulation of microvascular endothelial cell proliferation and migration, which results in neovascularization ${ }^{172,173}$.

PDGF is not only a strong mitogen, but also interacts with other factors. For example, it promotes fibrosis together with TGF- $\beta^{174}$, and induces active expression of MMPs. MMPs are a set of enzymes that are capable of degrading almost all kinds of protein components of the extracellular matrix (ECM). Therefore, it plays an important role in the process of tumor invasion and metastasis, as well as in angiogenesis in general.

\subsubsection{FGF Family}

FGF is a superfamily of proteins that is responsible for a variety of functions in metabolism, maintenance of organization structure during development as well as hemostasis. It has its effects through paracrine and autocrine signaling. Due to its high affinity to heparin, it is also known as the heparin binding growth factor $(\mathrm{HBGF})^{175}$. FGFs are found in both vertebrates and invertebrates. So far, at least 23 kinds of FGF proteins have been identified in humans and rats. It is divided into FGF-1 (FGF acidic) and FGF-2 (FGF basic) according to differences in structure, but both have effects in various biological processes such as 
angiogenesis, wound healing, cell proliferation, differentiation and cell survival $^{176}$.

FGFs' activity is mediated through four types of FGFRs, all with different coding genes. These four highly conserved RTKs (FGFR-1, FGFR-2, FGFR-3 and FGFR-4) are similar to most growth factor receptors ${ }^{177}$. FGFRs, a kind of transmembrane protein, contain an extracellular domain, a transmembrane domain and an intracellular domain, which provide the molecular basis of receptors for the binding of ligands and signal transduction ${ }^{178}$. Additionally, there is a decoy receptor, FGFR-5, which also binds to FGF ligands, but has no effect on proliferation $^{179}$.

Dimerization occurs when FGFRs bind to ligands, and phosphorylated FGFR tyrosine kinases further activate downstream pathways. Many experiments have shown that FGF and VEGF signaling, or other growth factor signal pathways such as PDGF have a number of important functions in angiogenesis, lymphangiogenesis and tumor metastasis ${ }^{180-183}$.

\subsection{Angiogenic Inhibitors}

Angiogenic inhibitors generate anti-angiogenic activity by affecting many different parts of the angiogenetic process, for example, ECM reconstruction, endothelial cell migration and proliferation, and microvascular lumen formation. Endogenous inhibitors of angiogenesis could be divided into two groups based on their different specifics. One type of angiogenic inhibitor act specifically on endothelial cells, and the other has effects on other cell types as well as endothelial cells ${ }^{184-186}$.

\subsubsection{Angiostatin}

In 1994 Angiostatin was first isolated from the serum and urine of a murine Lewis lung carcinoma model and was found to have a molecular weight of $38 \mathrm{kDa}$. Angiostatin acts specifically on endothelial cells, and inhibits their proliferation and migrations and induces apoptosis by binding to receptors on the surface of endothelial cells ${ }^{187,188}$.

Animal experiments have indicated that the therapeutic effect on tumors of angiostatin is stronger than common cytotoxic therapy. Its advantage is that angiostatin is appropriate for long-term usage because it does not display the 
acquired drug-resistance that is common. Currently, angiostatin has entered clinical trials in humans, and no dosage-limiting toxicity has been noted so far ${ }^{186}$.

\subsubsection{Endostatin}

Endostatin is an angiogenic inhibitor with a molecular weight of $20 \mathrm{kDa}$ that first was isolated and purified from plasma of mice with hemangioendothelioma in $1997^{189}$. Endostatin inhibits the migration of endothelial cells and influences survival ${ }^{190}$. Endostatin can also induce expression of anti-apoptotic proteins in endothelial cells by significantly reducing the level of pro-apoptotic factors leading to increased cell survival ${ }^{191}$. Furthermore, studies showed that endostatin can modulate multiple signaling pathways of vasculogenesis, including VEGF and FGF-induced signal transduction pathways ${ }^{192}$.

Endostatin down-regulates numerous key regulators in the pro-vasculogenesis signaling pathways such as $\mathrm{H} 1 \mathrm{~F}-1-\alpha$ gene transcription and reduces the expression levels of related factors in signaling pathways (i.e., VEGF and TNF- $\alpha$ ) at the same time. In addition, it induces dephosphorylation of various proteins related to the vasculogenesis signaling pathways to inhibit their functions. Endostatin negatively regulates angiogenic factors while positively regulating angiogenic inhibitors. These two effects act synergistically to inhibit the vasculogenesis.

Endostatin was the first blood vessel growth inhibitor entering clinical trials due to its strong anti-tumor activity. The results of previous clinical trials demonstrated the safety of endostatin. The strong endogenous factors endostatin and angiostatin inhibit growth, proliferation and migration of endothelial cells, and consequently restrain the growth of tumor cells and make them to enter a dormant state ${ }^{187,189}$.

\subsection{Antiangiogenesis Compounds}

Different from other traditional anti-tumor drugs, anti-angiogenic compounds target normal endothelial cells. They inhibit growth and metastasis of tumors. If tumors display drug-resistance to common cytotoxic therapy anti-angiogenic compounds may be the ideal drugs for treating cancer patients. FDA has approved several angiogenic inhibitors for tumor treatment in recent years, such 
as bevacizumab, sunibinib malate and sorafenib. Additionally, several angiogenic inhibitors are still under development and in the near future they will be evaluated in clinical trials.

\subsubsection{Bevacizumab}

Bevacizumab (trade name Avastin) is a humanized monocloncal antibody targeting VEGF-A, was developed by Genentech. It was approved by FDA in February 2004 and was the first VEGF inhibitor that came onto the market. Bevacizumab can selectively neutralize VEGF-A, but does not interact with other VEGF family members such as VEGF-B, VEGF-C, VEGF-D or VEGF-E. Moreover, bevacizumab specifically inhibits the biological function of VEGF-A, and thus influence mitogenic activity of endothelial cells, vascular permeability and vasculogenesis activity ${ }^{193}$. Preliminary clinical studies indicated that bevacizumab effectively could inhibit growth of several transplanted human tumor cells ${ }^{194}$, but in vitro research failed to prove the inhibitory effect on human tumor cell lines. This indicated that the effect of bevacizumab was only related to neovascularization ${ }^{195}$. The major adverse drug effects of bevacizumab include hypertension, rhinorrhagia, fever, and occasionally coagulation and hemorrhage that can be life-threatening ${ }^{196}$.

\subsubsection{Sunitinib Malate}

Sunitinib malate, (trade name Sutent), came into market in $2006^{197}$. Being an oral multiple-target tyrosine kinase inhibitor drug, sunitinib can selectively target some protein receptors (e.g., VEGFRs and PDGFRs ${ }^{198}$ ) that have a molecular switching function in the process of tumor growth. Sunitinib inhibits the growth and metastasis of tumor cells, significantly prolong the survival period and improve signs and symptoms cause by cancer. It can be applied widely and produces less adverse reactions than many other treatment options and is convenient for oral administration.

\subsubsection{Sorafenib Tosylate}

Sorafenib tosylate (trade name Nexavar) was approved by the FDA as an oral drug for treatment of late-stage renal carcinoma in December 2005. Sorafenib is a multiple kinase inhibitor with double anti-tumor effects. It inhibits numerous kinases including VEGFR-2, VEGFR soluble VEGFR-3 and PDGFR- $\beta$, and also 
blocks the Ras-Raf-MAPK signal pathway to inhibit proliferation of tumors directly. Indirectly it suppresses tumor growth by preventing the neovascularization of the tumor.

\subsubsection{VEGF-Trap}

sVEGFR is one of the alternative splicing forms of VEGFR in vivo. sVEGFR only contains the extracellular Ig-like domain of VEGFR. The artificial recombinant sVEGF is called 'Trap' and was developed by Regeneron. The second Ig-like domain of VEGFR-1 and the third Ig-like domain of VEGFR-2 were linked to the $\mathrm{Fc}$ fragment of human $\mathrm{IgG}$ by means of genetic engineering ${ }^{196,199}$. The recombinant protein has a longer half-life than the endogenous sVEGFR, and a higher binding affinity with VEGF ${ }^{199}$. Also compared with bevacizumab VEGF-Trap has a higher binding affinity with VEGF, and it also binds to VEGF-B and PIGF. Experiments in vitro showed that the VEGF-Trap inhibits growth of various tumor cells. A low dose of VEGFTrap produces similar efficacy levels as the humanized anti-VEGF monoclonal antibody. A high dose of VEGF-Trap not only inhibits growth of tumors, but also causes avascular tumors ${ }^{199}$.

Ongoing development of anti-angiogenic or anti-tumor drugs targeting VEGF/VEGFR includes, for example, the humanized anti-VEGFR-2's antibodies IMC-1C11, IMC-1121B ${ }^{200}$, and AG-013736 201.

\section{ZEBRAFISH ANIMAL MODEL}

The progress in medical and biological sciences is highly dependent of adequate animal models. Because embryology and genetics evolved through studies in drosophila, it became one of the most important models for molecular auxology of invertebrates. However, the structure of embryos and the pattern of development between invertebrates and vertebrate are rather different. Therefore, research of embryogenesis, embryonic development, occurrence and development of diseases in human or vertebrates should be carried out in a vertebrate model. Mice and rats are often appropriate models but there is a limitation in their use for embryonic studies as their embryos develops in the uterus of the parent, making visualization difficult. Fish, on the other hand, are 
gaining interest as a possible animal model for studying developmental biology due to a number of reproductive characteristics, such as their strong ability to reproduce, that spawning and fertilization can occur in vitro, and that the embryos are transparent. As a result, zebrafish are used as one of the most important representative vertebrates for research.

The first publication on zebrafish was published in 1981. In this publication the natural mutant "golden" was introduced in Nature ${ }^{202}$, and by 1996, about 4000 mutations in zebrafish had been identified ${ }^{203,204}$. Moreover, the sequencing of the zebrafish genome is almost completed. These rich genetic resources provide a solid basis for studies of molecular mechanisms in vertebrate growth. Due to available genetic manipulation techniques these circumstances gave the zebrafish model advantages. For example, morpholino oligos can be applied to knock down gene expressions, and delivery of mRNA can rescue this phenotype. Another advantage of using zebrafish embryos is their transparency and ex vivo development, which make them easy for microscopic analysis. Moreover, the diameter of the zygote is about $1 \mathrm{~mm}$, which allows for fine manipulation such as microinjection. Compared with mammals, zebrafish survives in a relatively hypoxic environment ${ }^{205,206}$, which is favorable for studies to observe physiological or pathological blood vessel growth in a hypoxic environment.

More importantly, zebrafish embryos develop rapidly. Eggs complete the first mitosis in 40 minutes after fertilization at $28.5^{\circ} \mathrm{C}$, and then divide once every 15 minutes. The major basic organs begin to develop just one day post fertilization. Three months later, zebrafish embryos reach sexual maturity. Lastly, it is convenient to breed and culture zebrafish with a large number of groups in a limited space. Adult zebrafish lay eggs once a week, each time spawning between 100-300 eggs. This makes the zebrafish a cost-effective model.

Overall, by combining optimal animal models for angiogenesis research and of VEGF research in particular it is possible to gain further insight of the mechanism that is important in health and disease. Ultimately this may lead to improved cancer treatments as well as identification of new treatment targets for other diseases. 


\section{AIMS OF THIS THESIS}

The overall aim of this thesis was to investigate the mechanism of VEGFmediated vascular functions in health and disease.

Specific aims:

1. To develop optimal animal models able to clarify mechanisms of angiogenesis during hypoxic conditions.

2. To evaluate the effects and functions of VEGF signaling in healthy vasculature.

3. To reveal the role of the circadian clock in VEGF-induced angiogenesis. 
Aims of this Thesis 


\section{METHODS}

\section{Zebrafish Model}

This thesis contains three manuscripts that utilise an adult zebrafish model, as well as zebrafish embryos because of their many advantages over other animal models for our purposes.

Because of the advantages that were described in the introduction section of this thesis, we used a strain $\operatorname{Tg}(f l i 1: E G F P)$ zebrafish line in paper I and paper II as the main experimental animal model which expresses a green fluorescent protein in the blood vessel's ECs. All embryos were produced by natural mating, and maintained in standard 0.3× Danieau's or E3 embryos medium at $28.5{ }^{\circ} \mathrm{C}$. Additionally, in paper IV, there are further studies using adult zebrafish as a model.

In paper I and II, use of an optical microscope (OM) enabled detachment of adult zebrafish retina and microinjections of tumor cells into the zebrafish embryos. A fluorescence microscope was used to perform qualitative and quantitative studies to identify the vasculature in fli1:EGFP zebrafish as well as tumor invasion and metastasis. The advantage is the ability to observe living cells dynamically using multiple fluorescent markers and track tumor dissemination using same fish at the different time points. Additionally, confocal microscope is also utilized in the experiments. Laser confocal scanning visualizes not only one layer of the image but also three-dimensional images, which provide detailed information of vessel structures and single tumor cells. This method is advantageous for studying angiogenesis.

\section{Hypoxic Chamber}

In order to create hypoxia environment for zebrafish in paper I and II, we designed a unique aquarium with a capacity of 1L. The hypoxic living environment was set up by adding nitrogen gas bubbles to water as very small bubbles and controlled by an oxygen regulator with oxygen concentration monitoring by an oxygen electrode. 


\section{Pharmacological Treatments and Morpholino Injection}

For the study purposes, we applied drug treatments in different animal models, including oral medication, such as a $\gamma$-secretase inhibitor (DAPT) and ZN323881 added to the water environment of the zebrafish. Furthermore, morpholino, as a molecule for the modification of gene expression, was injected into zebrafish yolk at the single cell level. Morpholinos act by occupying the binding sites of the mRNAs to block the other molecules binding site to specific nucleic acid sequences.

\section{PCR}

In zebrafish experiments, RT-PCR (reverse transcription-PCR) and qPCR (quantitative real time-PCR) techniques was used. Zebrafish embryos were homogenized in buffer and RNAs were prepared using RNA purification kit. The expression levels of mRNAs detected are zebrafish period2, zebrafish bmalla, zebrafish vegfaa, zebrafish -actin.

\section{ChIP}

Chromatin Immunoprecipitation (ChIP) is often used to study the interaction between proteins and DNA in cells. In this thesis' experiments, mouse fibroblast cells were used for the EZ-ChIP chromatin immunoprecipitation assay to determine binding of Bmall to the vegf promoter. A mouse periodl fragment, known to bind to Bmal1, was used as a positive control. In brief, $100 \mu \mathrm{l}$ of sonicated chromatin was used for each immunoprecipitation reaction. A rabbit anti-Bmal1 antibody and a non-immune rabbit $\mathrm{IgG}$ were used for immunoprecipitation. The purified DNA was used for quantitative PCR analysis.

\section{Mouse Model}

Mice possess a high level of sequence homology in a number of genes with humans. Therefore mouse models were used in the experiments for studying systematic effects of anti-angiogenic drugs on whole organisms. In paper III we used 8-12 week-old female C57 black 6 (C57/B16) mice.

Mice were injected with a rabbit anti-mouse VEGF-specific neutralizing antibody (BD0801) and a rat specific antibodies to mice VEGFR-1 antibody 
(MF1) and a rat specific antibodies to mice VEGFR-2 antibody (DC101) intraperitoneally (IP). The treatment schedules include:

1) Twice per week injection of the anti-VEGF antibody ( $5 \mathrm{mg} / \mathrm{kg}$ ), anti-VEGFR-

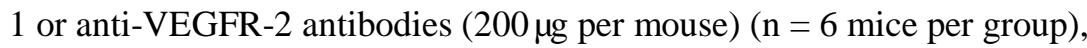

2) Twice per week injection of the anti-VEGF antibody $(5 \mathrm{mg} / \mathrm{kg})$ in the discontinuation experiment ( $\mathrm{n}=6$ mice per group) for 2 weeks. Tissues were collected at day $0,7,14$ and 28 after ending the anti-VEGF treatment $(n=6$ mice per group), and

3) Twice per week injection of different dosage of the anti-VEGF antibody (1.25, 5 , and $15 \mathrm{mg} / \mathrm{kg}$ ) ( $\mathrm{n}=6$ mice per group).

\section{Histological Studies and Immunohistochemistry}

In mouse experiments whole-mount staining and immunofluorescence techniques are used to visualize vessel structures in various organs. Collected tissue samples were fixed in $4 \%$ paraformaldehyde overnight and cut into thin slices followed by proteinase $\mathrm{K}(20 \mu \mathrm{g} / \mathrm{mL})$ treatment. The sliced samples were incubated with a rat anti-mouse CD31 antibody (1:200) overnight at $4{ }^{\circ} \mathrm{C}$, followed by staining with a goat anti-rat Alexa fluor 555 secondary antibody $(1: 200)$ for $2 \mathrm{~h}$ at room temperature. Slides were mounted using mounting medium (Vectashield) and vasculatures were visualized using a confocal laser microscope. Images were analyzed by Adobe Photoshop CS4 software. Paraffin-embedded tissues were sectioned at a thickness of $5 \mu \mathrm{m}$ and stained with a rat anti-mouse endomucin antibody (1:200) followed by a goat anti-rat Alexa fluor 555 secondary antibody (1:400). The slides were mounted using Vectashield and images were photographed using fluorescence microscopy equipped with a camera. H\&E staining were used for morphology studies. Samples were stained with hematoxylin followed by eosin after hydration of slides. Then slides were dehydrated and mounted. The images were obtained using light microscopy.

\section{Western Blot}

After 2 weeks anti-VEGF treatment, the bioreductive hypoxia marker pimonidazole $(1.5 \mathrm{mg} / \mathrm{mouse})$, was injected intravenously into the mice. They were then killed 15 minutes after the injection. The thyroid was collected and tissue lysate was used to assess levels of hypoxia. Proteins along with a protein ladder were subjected to SDS-PAGE followed by wet transferring onto 
methanol-activated polyvinylidene fluoride membranes. Membranes were blocked with $5 \%$ skim milk solution for $1 \mathrm{~h}$ before incubation with mouse antipimonidazole monoclonal antibodies overnight. Membranes were incubated for 45 min with a donkey anti-mouse IgG antibody was used. Positive signals were visualized using a chemiluminescence detection to identify the hypoxic indicated protein levels. Mouse $\beta$-actin was used as a loading control.

\section{Vascular Perfusion Assay}

At the end of vehicle- and anti-VEGF antibody treatment, mice were injected with a lysinated fluorescein-labeled dextran $(2,000 \mathrm{kDa}, 1 \mathrm{mg}$ in $100 \mu \mathrm{L}$ per mouse) and killed 10 minutes after injection. The thyroids were resected and fixed in $4 \%$ (wt/vol) PFA at $4{ }^{\circ} \mathrm{C}$ overnight and cut into small pieces under a light microscope. After proteinase $\mathrm{K}(20 \mu \mathrm{g} / \mathrm{mL})$ treatment, sliced samples were incubated with a rat anti-mouse CD31 antibody (1:200) overnight at $4{ }^{\circ} \mathrm{C}$, followed by staining with a goat anti-rat Cy5 secondary antibody (1:200) for $2 \mathrm{~h}$ at room temperature. Slides were mounted using mounting medium (Vectashield). Vasculatures and injected lysinated fluorescein-labeled dextran were visualized using a confocal laser microscope. The vessel area with dextran was calculated as a perfused area using Adobe Photoshop CS4 software.

\section{Elisa}

Blood from mice was collected by cardiac puncture and centrifuged at 2400 gravitational acceleration for 15 minutes. Collected serum samples were kept at $-20{ }^{\circ} \mathrm{C}$ until further use. Serum levels of mouse free triiodothyronine (T3) and free thyroxine (T4) were measured and quantified by using an ELISA method according to the manufacture's instruction (MSB 705057 and MSB162146; MyBiosource). In brief, sample or standard samples with known concentrations were placed into a 96 well plate followed by an incubation with conjugate proteins. After several washing, HRP-avidin and substrate solution were applied. Absorbance at $450 \mathrm{~nm}$ was detected by a spectrophotometer and free T3 and free $\mathrm{T} 4$ concentration were calculated. 


\section{RESULTS AND DISCUSSIONS}

\section{Adult zebrafish model for study of hypoxic retinopathy. (PAPER I)}

Pathological alterations and imbalance of metabolism in retinal vasculature will lead to tissue hypoxia and consequently induce angiogenesis ${ }^{207,208}$. Tissue hypoxia and angiogenesis will also result in retinopathy, which is a common cause of blindness. Therefore, using an animal model to study the mechanism behind this process is important to develop clinically relevant therapeutic approaches. The non-invasive animal model in the adult zebrafish developed in this thesis aims to mimic hypoxia-induced retinopathy observed in the clinic. This novel model has the main advantage of the ability to validate the efficacy of a water-soluble drugs added to the water.

To construct an adult zebrafish model for studying hypoxia-induced retinopathy, the first task was to consider the required equipment. The setup of the hypoxia facility should be simple and easily controlled in order to obtain accurate and reproducible results. It is of high importance to expose zebrafish to sufficient hypoxic conditions that are equivalent to the level of hypoxia that causes hypoxia-induced retinopathy seen in humans. It is, however, also necessary to ensure that the zebrafish can survive at these low oxygen levels.

Through continuous attempts and adjustments to obtain the optimal level of hypoxia and apparatus set up, a construct an adequate hypoxic state for adult zebrafish was established. To achieve this, we used a lidded but not sealed aquarium perfused with nitrogen to give uniform saturation of the water with nitrogen. The size of the aquarium is of a relatively small size (approximately 1 liter in volume) to enable appropriate administration of water-soluble drugs at later stages of these experiments. To certify the homogeneity of oxygen levels in the water and to decrease the imbalance of pressure produced by the nitrogen gas perfusion, a magnetic stirrer was placed at the bottom of the aquarium covered by a fine net. To accurately monitor and control the appropriate oxygen level in the zebrafish aquarium, the behavior of the fish was carefully observed and oxygen 
levels were adjusted accordingly. If their survival rate was low hygiene including water quality was prioritized. By adjusting to acute hypoxia, adult zebrafish developed hypoxia-induced vascular effects on retina after 3 to 10 days of hypoxic exposure.

At the experimental checkpoints, adult zebrafish was immediately fixed and the retina was isolated. The size of eye is around $2-3 \mathrm{~mm}$. Hence, proper size of tools and fine techniques under microscope was required. A rapid preparation process of microscopic work and imaging is obligatory to protect the green fluorescence signal produced by the flil:EGFP zebrafish. ( see Figure 3 )

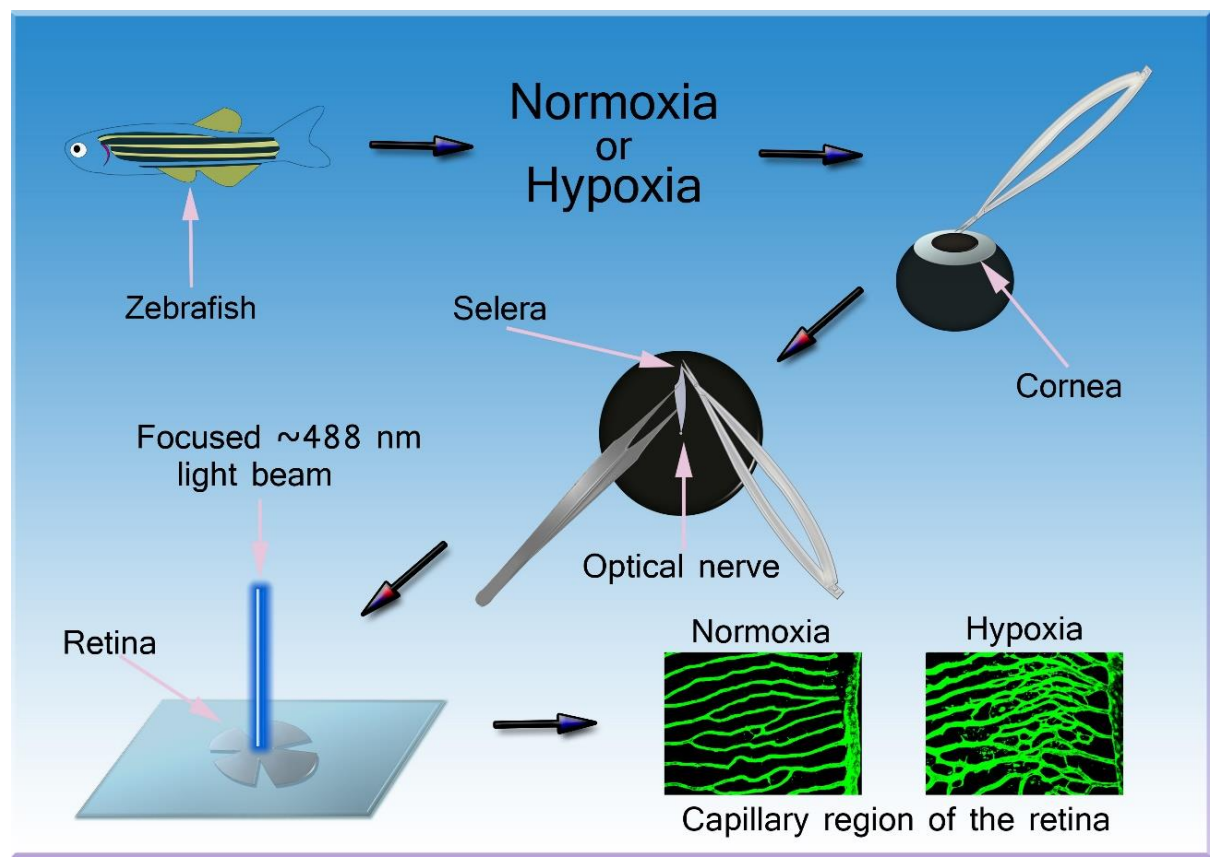

Figure 3. Critical steps of dissecting and analyzing the retinal vasculature in adult zebrafish.

The morphology of the blood vessel marked with green fluorescence can be clearly observed under fluorescent or confocal microscope after flat mounting of the isolated zebrafish retina. The vascular structure of the intact zebrafish retina is highly organized and divided into three regions. First is an artery region containing a central optic artery which consists of nearly 4 to 9 arteries feeding 2 to 4 branches. The second is a surrounding region with one circumferential vein at the edge of the retina. The third is a capillary region connecting to the arterial and venous region. 
At 6 days of exposure to hypoxia the retinal vasculature showed structuring tip cells in the arterial region, formation of capillary branches and more disorganized vascular structure in general. When keeping zebrafish longer in the hypoxic environment, the sprouting in arterial region disappeared. It might thus be a transient phenomenon caused by the acute hypoxic condition. For this thesis an quantification method able to evaluate the vascular alterations in zebrafish retina was created to precisely obtain scientifically solid differences in the statistical sense.

Therefor this thesis established, adult zebrafish model for studies of hypoxic retinopathy have certain advantages. The genetic tools available for zebrafish such as morpholinos and transgenic zebrafish are highly developed. Moreover, oral compounds, e.g. water-soluble chemical inhibitors and stimulators, can easily be distributed in the water. Additionally, it is feasible to tightly control the entire experimental process.

There are some limitations of this model. It cannot limit to exposure of hypoxia to individual organs. Regional hypoxia might be more relevant to certain clinical situations. Unfortunately there are few antibodies available, limiting the spectrum of immunohistochemical analysis in zebrafish. Moreover, a highly precise surgical technique to isolate retina is required. Taken together, however, this adult zebrafish hypoxia model of retinopathy provides us with the possibility to perform many experimental applications.

\section{Embryonic zebrafish model for mechanistic study of hypoxic metastasis. (PAPER II)}

Hypoxia is a potent inducer for the expression of VEGF, the most important regulatory factor in angiogenesis. Therefore hypoxia induces neovascularization in the tumor environment, which leads to dissemination of tumor cells into the circulation. This pathological angiogenesis is a crucial path in tumor metastasis formation. Although the size of the primary tumor is relatively small during early events of tumor development, tumor invasion and metastasis has already occurs at that time 209 . Current studies of the tumor metastatic cascade are mainly 
focused on the later stages of tumor development, yet the early stage is not detected in animal models. Utilizing the favorable biological characteristics of zebrafish, we were able to develop a metastatic model using embryonic zebrafish. This model allows us to observe single tumor cell dissemination, invasion and metastasis on monolayers of tumor cells in living organisms.

For this development a similar setup as in our adult zebrafish experiment was used (see Paper 1). Several details were modulated depending on the needs for the specific research questions. For example, PTU stock solution should be used for preventing pigment formation in zebrafish embryos during 24-48 hpf. Danieau's solution, the near-isotonic solutions for developing cell transplantation work for the zebrafish embryos, was also used. The oxygen level (7.5\%) in water was lower than in the adult zebrafish experiments, due to the fact that embryonic zebrafish tolerate hypoxia better than adults. The higher number (close to 100) of zebrafish embryos placed in hypoxia chamber was required because of a high death rate. Dead embryos exhibit an opaque white. Fortunately, dead embryos had little negative effect on the quality of the water and therefore dead embryos remained in the water until the end of experiments.

The age of $48 \mathrm{hpf}$ flil:EGFP-transgenic eggs were used for injection of tumor cells. Nearly 100 fluorescent Dil-labeled human or mouse tumor cells are carefully injected into the embryos. These numbers of tumor cells enable not only ensuring their survival in host, but not disturbing the development of zebrafish embryos due to excessive number of tumor cells. Perivitelline cavity is the preferable location of the transplanted tumor cells where we found. Perivitelline cavity is non-vascularized area so as to restrict the excessive speed of dissemination and invasion of tumor cells from the primary tumor mass into distal parts of the embryo body. After three days tumor implantation, the zebrafish embryos were taken out and examined under fluorescent and confocal microscopy. Implanted tumor cells under hypoxic conditions accomplished dissemination, invasion and metastasis, while the normoxia control group did not show tumor invasiveness.

This model provides an opportunity to study tumor cell invasion and metastasis under hypoxic conditions. The advantages of using zebrafish embryos for a tumor invasion assay are that they are transparent and immunoprivileged. The transparent nature of the fish permits visualization of vascular structures and 
tumor dissemination under the microscope. The fact that they are immunoprivileged allows implantation of mammalian tumor cells, including both human and mouse cells. Using morpholinos, target genes in zebrafish embryos are effectively knocked down. Additionally, drugs are dissolved in the water and can easily be administered to block or stimulate biological functions. Furthermore, by using DiI dye to visualize tumors, the observation window is extended up to 7 days. This time frame is sufficient to observe the entire process of tumor dissemination and invasion. The system developed in this thesis permits exposure of the zebrafish embryo to adequate hypoxia levels so that the model can assess hypoxia-induced or VEGF induced angiogenesis and tumor cell metastasis. Therefore, this model is able to test antiangiogenic compounds against new targets found in hypoxia induced angiogenesis and tumor cell migration.

There are some limitations of this model. For instance, the hypoxic area is not localized but affects the entire zebrafish embryo. It is impossible to create a $37^{\circ} \mathrm{C}$ condition that would be suitable for most mammalian tumors to grow. Zebrafish will not be able to survive at that temperature limiting the number of tumors that can be tested. As mentioned previously training is necessary to acquire the micro-operation skills needed to standardize analyses.

\section{VEGF blockades alter healthy vasculature in mice. (PAPER III)}

Anti-VEGF drugs like bevacizumab are commonly used today and are prescribed to cancer patients for treatment of various solid tumors at different locations in the body ${ }^{210-214}$. Clinicians have observed that these drugs not only have antiangiogenic effects but also produce a wide range of adverse effects including hypertension, renal vascular injury, gastrointestinal perforation and congestive heart failure ${ }^{215-217}$. The anatomical and structural basis of these adverse effects of anti-VEGF therapy is poorly understood.

This study investigated vascular alterations mediated by the anti-VEGF agents. These agents include an anti-VEGF neutralizing antibody (BD801), an antiVEGFR1 neutralizing antibody (MF-1) and an anti-VEGFR2 neutralizing antibody (DC101), which binds to VEGF, VEGFR1 and VEGFR2 respectively. The antibodies were administrated to healthy mice systemically for two weeks at 
a dose known to block tumor angiogenesis. The comparison of results is shown in Figure 4.

\begin{tabular}{|c|c|c|c|c|c|c|c|c|c|c|c|c|c|}
\hline & \multicolumn{3}{|c|}{ Endocrine organs } & \multicolumn{4}{|c|}{ Gastrointestinal tracts } & \multirow[b]{2}{*}{ Ovary. } & \multirow[b]{2}{*}{ Uterus } & \multirow[b]{2}{*}{ Liver } & \multirow[b]{2}{*}{$\begin{array}{c}\text { Pancreatic } \\
\text { acini }\end{array}$} & \multirow[b]{2}{*}{ Thymus } & \multirow[b]{2}{*}{$\begin{array}{l}\text { Renal } \\
\text { cortex }\end{array}$} \\
\hline & Thyroid & $\begin{array}{l}\text { Adrenal } \\
\text { cortex }\end{array}$ & $\begin{array}{c}\text { Pancreatic } \\
\text { islet }\end{array}$ & $\begin{array}{l}\text { Gastric } \\
\text { wall }\end{array}$ & $\begin{array}{c}\begin{array}{c}\text { Small } \\
\text { Intestine } \\
\text { wall }\end{array} \\
\end{array}$ & $\begin{array}{c}\text { Small } \\
\text { Intestine } \\
\text { villi }\end{array}$ & $\begin{array}{c}\text { Colon } \\
\text { wall }\end{array}$ & & & & & & \\
\hline Anti-VEGF-A & $* * *$ & $* * *$ & $* * *$ & $* *$ & $* *$ & $* *$ & * & $*$ & $* * *$ & $*$ & $* * *$ & $* *$ & $* * *$ \\
\hline Anti-VEGFR-1 & $* * *$ & $* * *$ & $* *$ & $*$ & $* *$ & * & * & $*$ & $* *$ & $*$ & $*$ & $* *$ & $* * *$ \\
\hline \multirow[t]{3}{*}{ Anti-VEGFR-2 } & ns & ns & ns & ns & ns & ns & ns & $*$ & $*$ & ns & ns & ns & ns \\
\hline & & \multicolumn{5}{|c|}{ Brain } & \multicolumn{3}{|c|}{ Muscle } & & & & \\
\hline & Glomerulus & $\begin{array}{c}\text { Olfactory } \\
\text { bulb }\end{array}$ & $\begin{array}{l}\text { Cerebral } \\
\text { cortex }\end{array}$ & $\begin{array}{l}\text { Hypotha- } \\
\text { lamus }\end{array}$ & Cerebellum & $\begin{array}{c}\text { Medulla } \\
\text { oblongata }\end{array}$ & Myocardium & Intrinsic & $\begin{array}{l}\text { Skeletal } \\
\text { muscle }\end{array}$ & $\begin{array}{c}\text { Bone } \\
\text { marrow }\end{array}$ & Retina & $\begin{array}{l}\text { Adrenal } \\
\text { medulla }\end{array}$ & \\
\hline Anti-VEGF-A & $*$ & ns & ns & ns & ns & ns & ns & ns & ns & ns & ns & ns & \\
\hline Anti-VEGFR-1 & ns & ns & ns & ns & ns & ns & ns & ns & ns & ns & ns & ns & \\
\hline Anti-VEGFR-2 & ns & ns & ns & ns & ns & ns & ns & ns & ns & ns & ns & ns & \\
\hline
\end{tabular}

Figure 4. Impact of Anti-VEGF blockades on vasculature of healthy mice.

The endocrine organs, including the thyroid, adrenal cortex and pancreatic islets, are known to produce high levels of VEGF. Therefore we first examined these organs to analyze vascular alterations by administration of anti-VEGF therapy. Upon anti-VEGF treatment, the thyroid showed a reduced vascular density by almost $60 \%$ in the examined whole-mount staining preparations. A similar degree of vascular regression was consistently observed in the other endocrine organs. No significant structural changes were observed by the H\&E staining after anti-VEGF treatment. The VEGFR-2 blockade showed an impact on vascular regression in these endocrine organs, while VEGFR1 blockade did not show any vessel regression. These findings pinpointed the important role of VEGF in maintenance of the vascular homeostasis on healthy vessels, including preservation of vascular density. VEGFR-2 was conformed to be the primary functional VEGF receptor involved in vessel maintenance, whereas VEGFR-1 appear to function as a decoy receptor ${ }^{94,218}$.

Secondly the gastrointestinal (GI) tract, the female reproductive system, renal cortex and glomeruli in the kidney, hepatic sinusoidal in the liver and pancreatic acini were examined in the same way as the endocrine organs. The data in Paper III showed that VEGF and VEGFR2 blockades also reduced vessel density in these organs, although the degree of vessel regression was not as high as what was seen in endocrine organs. Similar to the findings in the endocrine organs, 
VEGFR-1 blockade did not produce any vascular regressive effect. Again, no structural changes were observed from the H\&E staining.

Finally, the brain, retina, myocardium, skeletal muscles and bone marrow were examined. Importantly, the vasculature in these organs did not show significant response to any anti-VEGF blockades.

As an additional experiment the thyroid was thoroughly investigated as an offtarget effect of the administered agent as the vasculature in this organ was affected most by the anti-VEGF treatment. Healthy mice were administrated an anti-VEGF agent for two weeks followed by anti-VEGF cessation. The vessel structure, apoptotic impact on endothelial cells, hypoxia and tissue VEGF level were analyzed. After two weeks of blockade withdrawal vascular regrowth was observed, eventually with nearly normalized vascular density. The results indicate that the vascular regression produced by anti-VEGF treatment is due to decreased endothelial cell survival maintained by VEGF and it is a reversible process. The recovery of the ability of vascular regression in a short time span may have been an effect of tissue hypoxia inducing high expression levels of VEGF to stimulate the angiogenic process. This demonstrates that VEGF is a key factor for vessel maintenance.

Research has demonstrated that systemic delivery of anti-VEGFR component caused vascular regression in various tissues ${ }^{219}$. To study the functional impacts of anti-VEGF treatments on the thyroid, measurements of the blood vessel perfusion and leakiness was performed by using dextran dye in several organs from both anti-VEGF treated and untreated groups. The experimental data demonstrated anti-VEGF agents induced a significant decrease in blood leakage from vessels, but no alteration of blood perfusion, indicating VEGF is an important permeability factor in healthy organs. Subsequently, the serum levels of the thyroid hormone, free T3 and free T4, were detected by ELISA. Free T4 but not free T3 was lower in the anti-VEGF treated group compared to the untreated group. We further examined alterations of vascular fenestrations (openings in the blood vessel wall) in the thyroid upon anti-VEGF treatment, due to the fact that the vascular fenestrations are an important feature for hormone secretion by endocrine organs. As expected, highly fenestrated blood vessels in the untreated group turned to non-fenestrated vessels in the anti-VEGF treated group. Interestingly, more endothelial caveolae was observed in the anti-VEGF treated group. One possible explanation for this observation is that they are 
produced to compensate for the suppression of vascular fenestrations to maintain normal hormone secretion in the thyroid.

Taken together, the blockage of the VEGF signaling pathway induces a series of vascular alterations including a reduction in vascular density. Consequently, the vessel and organ functions are in part impaired by anti-VEGF therapies. In a clinical setting anti-VEGF treatment targets tumor sites as well as healthy organs. The effect of anti-VEGF treatment might thus be different between cancer and healthy cells ${ }^{216,217}$. In addition, cancer patients need to be dosed with anti-VEGF agents for long time, or several cycles with cessation. The adverse effects on healthy organs might be more serious and their function might therefore become irreversible unless some time is allowed for vessel structure to be regained ${ }^{220,221}$. Tumor sites might develop to be more aggressive due to hypoxia or change of angiogeneic profiles are a response to anti-VEGF therapy. These vital points should be addressed using our preclinical mice models in the future.

\section{Discovery of mechanisms underlying circadian clock control of angiogenesis in embryonic zebrafish model. (PAPER IV)}

We investigated the role of the circadian clock in angiogenesis and found there is a coupling of these two factors in the developmental stage of the zebrafish. Importantly we focused on two powerful transcription factors regulating the circadian clock, namely Bmall and Period2, and their interaction with an angiogenic factor, VEGF.

The impact of the circadian clock on developmental angiogenesis was studied in $\operatorname{Tg}$ (Flil:egfp) zebrafish embryos under three different conditions; constant light (LL), constant darkness (DD) and switching between $12 \mathrm{~h}$ light and $12 \mathrm{~h}$ dark (LD). At the age of 1 hour postfertilization ( $\mathrm{hpf}$ ), the embryos started developing under identical conditions and were visually examined at different time points with regard to their development including viability, phenotype, somite lengths and head angles (two commonly used parameters for staging of the development of zebrafish embryos). Different levels of light exposure did not result in any significant alterations in the studied parameters. However, quantification analysis of developmental vessels showed that LL exposed zebrafish embryos at $72 \mathrm{hpf}$ 
relative to $24 \mathrm{hpf}$ possessed decreased development of intersegmental vessels (ISVs) and subintestinal veins (SIVs) as compared with LD exposed zebrafish embryos. DD exposure did not alter ISVs and SIVs as compared with LD exposure. Importantly, the impaired angiogenesis produced by disrupted circadian clock (LL condition) did not affect the development of zebrafish embryos. One possible explanation is that zebrafish takes up oxygen and nutrients through free diffusion instead of supply from functional blood vessels. Another possible explanation may be zebrafish have acquired compensatory mechanisms by having many clock gene homologs. If one clock gene were impaired, another clock gene could compensate through regulation to rescue the defects caused by the failed clock gene.

The circadian control on angiogenesis was further investigated utilizing morpholinos specific to the circadian clock genes bmall and period 2 as a loss of function. Under LD, injection of a bmal1 morpholino decreased ISV growth while injection of a period 2 morpholino increased ISV growth as compared with controls. Under LL, injection of a bmall morpholino produced no additive effect on the suppression of ISV growth, while injection of a period 2 morpholino abolished the suppression of ISV growth and normalized the ISV growth. This demonstrated that Bmal1 and Period2 displayed opposing effect on angiogenesis. Next, as a gain of function approach, bmall and period 2 mRNAs were injected into the zebrafish embryos. Co-injection of a bmall morpholino with bmall mRNA resulted in nearly normalized ISV defect. LL exposure together with a bmall mRNA injection also restored LL induced ISV inhibition. Furthermore, co-injection of a period 2 morpholino with period 2 mRNA resulted in abolition of period 2 morpholino-mediated ISV growth. These results provided evidence of the specificity of bmall and period 2 morpholinos and confirmation on their opposing angiogenic effects.

To study the role of VEGF in circadian regulated developmental angiogenesis, the zebrafish embryos were injected with combination of vegf, bmall or period 2 morpholinos. Injection of vegf morpholino alone to zebrafish embryos led to decreased ISV growth in a dose dependent manner. Co-injection of vegf and bmall morpholinos resulted in additively decreased ISV growth. On the other hand, co-injection of vegf and period 2 morpholinos resulted in slightly increased ISV growth but not an additive effect. Two orally active VEGFR tyrosine kinase inhibitors (TKIs), namely ZN323881 and sunitinib, experimentally displayed the 
same effect on regulation of ISV growth as the vegf morpholino. Taken together, these results demonstrated that Bmal1 but not Period2 mediates VEGF-induced angiogenesis.

The molecular mechanism underlying how Bmall interacts with vegf upon regulation of angiogenesis was also studied. The hypothesis was that Bmall may directly target the promoter region of vegf. Therefore, a system able to detect the bmall and vegf promotor activities using a luciferase reporter system in zebrafish embryos under various light source exposures was employed. Firstly, vegf promoter activity was measured under LD or LL condition. As theorized, the vegf promoter activity significantly decreased under LL condition. We further investigated vegf promoter activity in bmall morpholino-treated zebrafish embryos. Both zebrafish vegf and human vegf promoter activity were reduced by bmall morpholino treatment indicating that Bmall directly regulates the promoter region of vegf. Furthermore, the zebrafish vegf promoter activity displayed a dependence on circadian rhythm under LD and DD exposures, while LL exposure diminished zebrafish vegf promoter activity and circadian rhythmicity. Measurement of mRNA levels of bmall and vegf using qPCR under LD and DD conditions demonstrated circadian rhythmicity, whereas LL exposure disrupted it. These results demonstrate that expression of VEGF and its circadian rhythmicity is regulated by circadian clock gene bmall. Additionally, we analyzed the potential Bmal1-binding E-boxes in the promoter regions of vegf genes in zebrafish, mice and humans. Deletion of the E-boxes in the promotor region of zebrafish resulted in significantly decreased and even abolished zvegf promotor activity. Finally, we performed a chromatin immunoprecipitation assay (a classical method to detect protein and DNA binding) and found that Bmall directly binds to E-boxes in the promotor region of vegf.

Moreover, the relationship between the notch signaling pathway and circadian clock on developmental angiogenesis was investigated.

Inhibition of notch signaling resulted in increased but disorganized growth of ISV in the zebrafish embryo. Administration of a vegf morpholino reduced the notch inhibition-induced excessive growth of ISV. This demonstrates VEGF regulates notch-induced vascular changes. Importantly the bmall morpholino reduced notch inhibition-induced excessive growth of ISV, while the period2 morpholino showed no such effect. Even the LL condition resulted in reduction 
of notch inhibition-induced excessive ISV growth. The period2 morpholino together with LL condition showed an increase in the notch inhibition-induced excessive growth of ISV. These results suggested that circadian clock-induced VEGF acts as the driving force for notch-induced angiogenesis.

This paper provides the first evidence that there is a relationship between circadian clock genes and angiogenesis in the developing zebrafish. It would be very important to further investigate the role of circadian clock genes in diseases - in particular cancer development, tumor growth and metastasis, and drug resistance in cancer patients, as well as healthy vascular maintenance. 


\section{CONCLUSIONS}

This thesis elucidates VEGF-mediated angiogenesis in physiological and pathological conditions using zebrafish and mice models.

In conclusion:

1. It is possible to develop zebrafish models using embryos and adults to study mechanisms of angiogenesis under hypoxia.

2. The embryonic zebrafish model demonstrates the feasibility of studying tumor metastasis at the early stage of metastatic events as well as hypoxiainduced metastasis in real time.

3. The adult zebrafish model permits evaluation of angiogenic mechanisms in hypoxia-induced retinopathy.

4. Inhibition of VEGF signaling in healthy mice alters the mature vasculature and in part impairs function in some organs.

5. The main receptor for VEGF signaling that maintains healthy vasculature is VEGFR2.

6. The circadian clock has an important role in the regulation of developmental angiogenesis in an embryonic zebrafish model.

Circadian clock genes bmall and period2 are involved in developmental angiogenesis, and bmall directly regulates VEGF production. 


\section{FUTURE PERSPECTIVES}

This thesis' work has paved new conceptual and methodological avenues for understanding the fundamental mechanisms of angiogenesis in physiology and pathology. Based on our zebrafish cancer metastatic model, we can reasonably speculate that this model could potentially be used to predict the metastatic potential and risk in a given cancer type in patients. To date, clinical assessments of cancer invasion and metastasis are largely based on observational data in patients and pathological properties of malignant cells per se. However, a functional in vivo model to study the metastatic potentials in living animals has been lacking. Thus our findings provide a functional system that can detect cancer cell behavior at the single cell level. Consistent with this notion, we have correlated the cancer invasion in the zebrafish model and the metastatic disease in a limited number of cancer patients. Our preliminary findings show that highly metastatic cancer types can be accurately predicted in our zebrafish model ${ }^{222}$. It would be interesting to expand this type of studies in the future and hopefully this functional assay could be routinely used in the clinic in combination with observational clinical data when needed. Future studies based using this zebrafish metastasis model should also be able to recapitulate the tumor microenvironment in which various host cells can be included in the metastatic assays. To this end, we have shown that the tumor-associated microphages, especially the M2 microphage subpopulation, are crucial for promoting cancer metastasis $^{222}$. In line with this we plan to develop multi-color-coded system to label various cells including tumor cell, macrophage, stromal fibroblast, endothelial cell, and perivascular cells with different color to study their role in facilitating cancer metastasis. Genetic zebrafish models in combination with color-coded isolated cells should then be used. With all this information available, our zebrafish cancer model can also be used to evaluate the role of various signaling molecules affecting metastasis formation. Accordingly, one of the most important advantages of our system is that we can study the metastatic events that the early stage of cancer development.

In the retina neovascularization zebrafish model, we have demonstrated and analyzed hypoxia-induced retinal angiogenesis in a non-invasive adult system. To the best of our knowledge, this is the first non-invasive and non-genetically 
manipulated retinal angiogenesis model in adult animals. Exposure of adult zebrafish in hypoxic water can be easily performed and zebrafish tolerate well to hypoxic environment. With this system, it would be interesting to screen for potential drugs that interfere with retinal neovascularization. As hypoxia is very common driving force for neovascularization, our model system is clinically relevant. This model can possibly be further developed for use under diabetic conditions. We hope that it will provide a reliable platform for discovery of new drugs that target retinal angiogenesis and these potential drugs will be used for treatment of millions of patients with diabetic retinopathy.

The clinical implication of "off-tumor" targets of anti-VEGF drugs is to understand the mode of actions of these drugs and their potential side effects. Particularly, for cancer patients who have pathological changes in one tissue where the anti-VEGF drug targets most vasculature it is of great importance. Particular attention should be given adverse events to optimize safety for patients. We believe that our findings in mouse models accurately predict the antiangiogenic drug effects in human cancer patients. Indeed, hypertension and hypothyroidism are common side effects of anti-VEGF drugs and these clinically observed adverse effects correlate well with these drug-induced vasculature changes in thyroid and kidney.

The groundbreaking discovery that the circadian clock is of importance in the regulation of angiogenesis has stimulated our thinking to expand our efforts to pathological conditions such as cancer. It would be pivotally important to study if the disruption of clock genes in mice could cause drug resistance. If so, we need to develop a spate line of medication in combination with antiangiogenic drugs to improve therapeutic efficacy. Moreover, timing of any anti-cancer agent could be optimized. This interesting and important possibility warrants future study and validation.

Taken together, our findings presented in this thesis have paved many different new lines of possibilities for treatment of cancer and non-malignant diseases. If any of our approach would be successful in the future, it would be beneficial for millions of patients. 


\section{ACKNOWLEDGEMENTS}

This thesis was written in the Department of Medical and Health Sciences (IMH), Linköping University and Department of Microbiology, Tumor and Cell Biology (MTC), Karolinska Institutet. In the two departments, I spent the whole study course of Ph.D., which was one of the most an important experiences in my life. With the help of my supervisors, colleagues and friends, I happily and successfully accomplished my study. Here, I would like very much to present my special thanks to all who all you who have guided, accompanied and helped me during this important journey in my life.

First and foremost, I would like to show my deepest gratitude to my main supervisor, Professor Eric Wahlberg, a respectable, responsible and resourceful scholar who has provided me with valuable guidance at every stage of my Ph.D. study. Without your enlightening instruction, impressive kindness and patience, I could not have completed my thesis. Your keen and vigorous academic observation enlightens me not only in this thesis but also in my every step of the road of scientific research.

I would also like to extend my thanks to my co-supervisor, Professor Yihai Cao for all his kindness and help. He provided me with the opportunity to work and study in his laboratory, where I learnt a lot of knowledge and experimental skills - especially the ability to develop methods and solve problems.

My co-supervisor, Professor Toste Länne: I am so lucky to be a student of yours. You helped me to develop the fundamental and essential academic competence. I must offer my heartfelt thanks to you.

My co-supervisor, Dr. Kayoko Hosaka, words can't express my innermost feelings of gratitude to you. Thank you for the strongest support and academic guidance you have shown me throughout my study. Without your help I could not have made it today.

Dear Dr. Lasse Jensen, although you are not my co-supervisor, you taught and helped me from the moment I entered the laboratory. You are the first one to 
teach me experimental techniques using zebrafish. Your serious scientific attitude and rigorous scholarship deeply influenced and inspired me. Many thanks to you!

Dr. Xiaojuan Yang, thank you very much for sharing your research experience with me. I will never forget what you have given to me in these years. Thank you so much again for your suggestions, encouragement and all the great help you gave me.

Dear Dr. Sharon Lim, you always gave me help from from becoming a Ph.D student in Sweden until now. I really appreciate that you rent your house to me when I would have been out on the street. You have left me with many gorgeous memories during this period.

Marcus Ladds, I am very pleased and lucky to have met you at the end, right before I am going to graduate, thank you very much for your selfless help.

During the years of my study career, I had the honor to study and work together with many colleagues: Dr. Yin Zhang, Dr. Eva-maria Hedlund, Dr. Yunlong Yang, Dr. Takahiro Seki, Dr. Mitsuhiko Abe, Dr. Renhai Cao, Dr. Masaki Nakamura, Dr. Mei Dong, Dr. Hideki Iwamoto, Dr. Funeng Jiang, Dr. Pegah Rouhi, Dr. Yuan Xue, Dr. Danfang Zhang, Dr. Hong Ji, Patrik Andersson, Dr. Jennifer Honek, Carina Fischer, Jian Wang, Dr. Meili Sun, Qi Dang, you have been helpful to me in different ways in my life and study. Every one of you has already left a deep imprint in my heart. I cannot help extending my thanks to you all here too.

Beside our group, I still have a lot of friends to be thank, such as Dr. Hong Jin, Professor Lexun Xue, Dr. Beibei Xu, and Dr. Xuetao Wang, all of you gave me a lot of help not only with my academic study but also in life and faith.

I want to thank the staff of Linköping University, Elin Wistrand, Kaisa Bendtsen, Elisabeth Skargren, Malin Strand, Lisa Floxner, Tomas Hägg, Lisa Floxner and Martin Pettersson. I also want to thank the staff of Karolinska Institutet, Marlene Brink-Sinervo, Magnus Bjurström, David Meyer, Torbjörn Laggar, Per Persson, Helene Stambeck, Lina Rejnö and John Sennett. All of you helped me and instructed me patiently, making sure I got the required information and support. I feel very grateful to you! 
Now I shall conclude my study. During my life, many people have played important roles. Professor Mingxia Wu, Granny Liu, Granny Xu, Grandpa Tianen Li and Ms. Colier, as my seniors, you play an irreplaceable part as my family throughout my whole life. Wherever I am, you are always in my heart.

At the same time I also want to thank my wife Yanxin Chen, you are the most precious to me. I can honestly say that it is your determination and constant encouragement that ultimately make it possible for me. I gratefully thank my Father and and Mother. You provide me with unconditional love and care. Dear mom, I will never ever forget what you taught me: “至于我和我家, 我们必定侍奉耶和 华”! I would also like to thank all my relatives, who have supported me throughout this entire process. I know I always have you to count on when times are tough. Thank you for all your love!!! 


\section{REFERENCES}

1 Ware, J. A. \& Simons, M. Angiogenesis in ischemic heart disease. Nat Med 3, 158-164 (1997).

2 Ferrara, N. \& Alitalo, K. Clinical applications of angiogenic growth factors and their inhibitors. Nat Med 5, 1359-1364, doi:10.1038/70928 (1999).

3 Beck, L., Jr. \& D'Amore, P. A. Vascular development: cellular and molecular regulation. FASEB J 11, 365-373 (1997).

4 Hershey, J. C. et al. Revascularization in the rabbit hindlimb: dissociation between capillary sprouting and arteriogenesis. Cardiovasc Res 49, 618625 (2001).

5 Helisch, A. \& Schaper, W. Arteriogenesis: the development and growth of collateral arteries. Microcirculation 10, 83-97, doi:10.1038/sj.mn.7800173 (2003).

6 Risau, W. Mechanisms of angiogenesis. Nature 386, 671-674, doi:10.1038/386671a0 (1997).

7 Folkman, J., Merler, E., Abernathy, C. \& Williams, G. Isolation of a tumor factor responsible for angiogenesis. J Exp Med 133, 275-288 (1971).

8 Folkman, J. Tumor angiogenesis: therapeutic implications. $N$ Engl J Med 285, 1182-1186, doi:10.1056/NEJM197111182852108 (1971).

9 Cavallo, T., Sade, R., Folkman, J. \& Cotran, R. S. Tumor angiogenesis. Rapid induction of endothelial mitoses demonstrated by autoradiography. J Cell Biol 54, 408-420 (1972).

10 Brem, S., Cotran, R. \& Folkman, J. Tumor angiogenesis: a quantitative method for histologic grading. J Natl Cancer Inst 48, 347-356 (1972).

11 Bergers, G. \& Benjamin, L. E. Tumorigenesis and the angiogenic switch. Nat Rev Cancer 3, 401-410, doi:10.1038/nrc1093 (2003).

12 Florey. The endothelial cell. Br Med J 2, 487-490 (1966).

13 Giordano, F. J. \& Johnson, R. S. Angiogenesis: the role of the microenvironment in flipping the switch. Curr Opin Genet Dev 11, 35-40 (2001).

14 Blagosklonny, M. V. Antiangiogenic therapy and tumor progression. Cancer Cell 5, 13-17 (2004).

15 Lichtenbeld, H. C., Ferarra, N., Jain, R. K. \& Munn, L. L. Effect of local anti-VEGF antibody treatment on tumor microvessel permeability. Microvasc Res 57, 357-362, doi:10.1006/mvre.1998.2140 (1999).

16 Hannink, M. \& Donoghue, D. J. Structure and function of platelet-derived growth factor (PDGF) and related proteins. Biochim Biophys Acta 989, 110 (1989).

17 Ogasawara, S. et al. Expression of angiogenic factors, basic fibroblast growth factor and vascular endothelial growth factor, in human biliary tract carcinoma cell lines. Hepatol Res 20, 97-113 (2001). 
18 Ornitz, D. M. \& Itoh, N. Fibroblast growth factors. Genome Biol 2, REVIEWS3005 (2001).

19 Benedito, R. et al. Notch-dependent VEGFR3 upregulation allows angiogenesis without VEGF-VEGFR2 signalling. Nature 484, 110-114, doi:10.1038/nature10908 (2012).

20 Dorrell, M. I. \& Friedlander, M. Mechanisms of endothelial cell guidance and vascular patterning in the developing mouse retina. Prog Retin Eye Res 25, 277-295, doi:10.1016/j.preteyeres.2006.01.001 (2006).

21 Phng, L. K. \& Gerhardt, H. Angiogenesis: a team effort coordinated by notch. Dev Cell 16, 196-208, doi:10.1016/j.devcel.2009.01.015 (2009).

22 Hillen, F. \& Griffioen, A. W. Tumour vascularization: sprouting angiogenesis and beyond. Cancer Metastasis Rev 26, 489-502, doi:10.1007/s10555-007-9094-7 (2007).

23 Cao, Y. Angiogenesis: What can it offer for future medicine? Exp Cell Res 316, 1304-1308, doi:10.1016/j.yexcr.2010.02.031 (2010).

24 Kerbel, R. \& Folkman, J. Clinical translation of angiogenesis inhibitors. Nat Rev Cancer 2, 727-739, doi:10.1038/nrc905 (2002).

25 Zetter, B. R. Angiogenesis and tumor metastasis. Annu Rev Med 49, 407424, doi:10.1146/annurev.med.49.1.407 (1998).

26 Boehm, T., Folkman, J., Browder, T. \& O'Reilly, M. S. Antiangiogenic therapy of experimental cancer does not induce acquired drug resistance. Nature 390, 404-407, doi:10.1038/37126 (1997).

27 Folkman, J. Angiogenesis in cancer, vascular, rheumatoid and other disease. Nat Med 1, 27-31 (1995).

28 Cao, Y. Angiogenesis in malignancy. Semin Cancer Biol 19, 277-278, doi:10.1016/j.semcancer.2009.11.001 (2009).

29 Polverini, P. J. Angiogenesis in health and disease: insights into basic mechanisms and therapeutic opportunities. J Dent Educ 66, 962-975 (2002).

30 Bikfalvi, A. Angiogenesis: health and disease. Ann Oncol 17 Suppl 10, x65-70, doi:10.1093/annonc/md1239 (2006).

31 Yoo, S. Y. \& Kwon, S. M. Angiogenesis and its therapeutic opportunities. Mediators Inflamm 2013, 127170, doi:10.1155/2013/127170 (2013).

32 Schwesinger, C. et al. Intrachoroidal neovascularization in transgenic mice overexpressing vascular endothelial growth factor in the retinal pigment epithelium. Am J Pathol 158, 1161-1172, doi:10.1016/S00029440(10)64063-1 (2001).

33 Crawford, T. N., Alfaro, D. V., 3rd, Kerrison, J. B. \& Jablon, E. P. Diabetic retinopathy and angiogenesis. Curr Diabetes Rev 5, 8-13 (2009).

34 Ng, E. W. \& Adamis, A. P. Targeting angiogenesis, the underlying disorder in neovascular age-related macular degeneration. Can J Ophthalmol 40, 352-368, doi:10.1016/S0008-4182(05)80078-X (2005).

35 Anchala, A. R. \& Pasquale, L. R. Neovascular glaucoma: a historical perspective on modulating angiogenesis. Semin Ophthalmol 24, 106-112, doi:10.1080/08820530902800959 (2009).

36 Chen, J. \& Smith, L. E. Retinopathy of prematurity. Angiogenesis 10, 133- 
140, doi:10.1007/s10456-007-9066-0 (2007).

37 Lee, P., Wang, C. C. \& Adamis, A. P. Ocular neovascularization: an epidemiologic review. Surv Ophthalmol 43, 245-269 (1998).

38 Klein, R., Klein, B. E. \& Moss, S. E. Visual impairment in diabetes. Ophthalmology 91, 1-9 (1984).

39 Ghafour, I. M., Allan, D. \& Foulds, W. S. Common causes of blindness and visual handicap in the west of Scotland. Br J Ophthalmol 67, 209-213 (1983).

40 Yang, X. M. et al. Role of PI3K/Akt and MEK/ERK in mediating hypoxia-induced expression of HIF-1 alpha and VEGF in laser-induced rat choroidal neovascularization. Invest Ophthalmol Vis Sci 50, 1873-1879, doi:10.1167/iovs.08-2591 (2009).

41 Sivak-Callcott, J. A., O'Day, D. M., Gass, J. D. \& Tsai, J. C. Evidencebased recommendations for the diagnosis and treatment of neovascular glaucoma. Ophthalmology 108, 1767-1776; quiz1777, 1800 (2001).

42 Tripathi, R. C., Li, J., Tripathi, B. J., Chalam, K. V. \& Adamis, A. P. Increased level of vascular endothelial growth factor in aqueous humor of patients with neovascular glaucoma. Ophthalmology 105, 232-237 (1998).

43 Hurwitz, H. et al. Bevacizumab plus irinotecan, fluorouracil, and leucovorin for metastatic colorectal cancer. $N$ Engl J Med 350, 2335-2342, doi:10.1056/NEJMoa032691 (2004).

44 Bakri, S. J. et al. Six-month stability of bevacizumab (Avastin) binding to vascular endothelial growth factor after withdrawal into a syringe and refrigeration or freezing. Retina 26, 519-522, doi:10.1097/01.iae.0000225354.92444.7a (2006).

45 Margolis, R., Lowder, C. Y., Sears, J. E. \& Kaiser, P. K. Intravitreal bevacizumab for macular edema due to occlusive vasculitis. Semin Ophthalmol 22, 105-108, doi:10.1080/08820530701420074 (2007).

46 Byeon, S. H., Kwon, Y. A., Oh, H. S., Kim, M. \& Kwon, O. W. Short-term results of intravitreal bevacizumab for macular edema with retinal vein obstruction and diabetic macular edema. J Ocul Pharmacol Ther 23, 387394, doi:10.1089/jop.2007.0012 (2007).

47 Maia, O. O., Jr., Bonanomi, M. T., Takahashi, W. Y., Nascimento, V. P. \& Takahashi, B. S. Intravitreal bevacizumab for foveal detachment in idiopathic perifoveal telangiectasia. Am J Ophthalmol 144, 296-299, doi:10.1016/j.ajo.2007.03.059 (2007).

48 Mandal, S., Venkatesh, P., Sampangi, R. \& Garg, S. Intravitreal bevacizumab (Avastin) as primary treatment for myopic choroidal neovascularization. Eur J Ophthalmol 17, 620-626 (2007).

49 Yanyali, A., Aytug, B., Horozoglu, F. \& Nohutcu, A. F. Bevacizumab (Avastin) for diabetic macular edema in previously vitrectomized eyes. Am J Ophthalmol 144, 124-126, doi:10.1016/j.ajo.2007.02.048 (2007).

50 Amselem, L. et al. Intravitreal bevacizumab (Avastin) injection in ocular ischemic syndrome. Am $J$ Ophthalmol 144, 122-124, doi:10.1016/j.ajo.2007.02.037 (2007).

51 Shah, P. K., Narendran, V., Tawansy, K. A., Raghuram, A. \& Narendran, 
K. Intravitreal bevacizumab (Avastin) for post laser anterior segment ischemia in aggressive posterior retinopathy of prematurity. Indian $J$ Ophthalmol 55, 75-76 (2007).

52 Heiduschka, P. et al. Penetration of bevacizumab through the retina after intravitreal injection in the monkey. Invest Ophthalmol Vis Sci 48, 28142823, doi:10.1167/iovs.06-1171 (2007).

53 Rosenfeld, P. J. et al. Ranibizumab for neovascular age-related macular degeneration. $N$ Engl J Med 355, 1419-1431, doi:10.1056/NEJMoa054481 (2006).

54 Wolf, S. et al. RADIANCE: a randomized controlled study of ranibizumab in patients with choroidal neovascularization secondary to pathologic myopia. Ophthalmology 121, 682-692 e682, doi:10.1016/j.ophtha.2013.10.023 (2014).

55 Hanahan, D. \& Weinberg, R. A. Hallmarks of cancer: the next generation. Cell 144, 646-674, doi:10.1016/j.cell.2011.02.013 (2011).

56 Cao, Y. Antiangiogenic cancer therapy. Semin Cancer Biol 14, 139-145, doi:10.1016/j.semcancer.2003.09.018 (2004).

57 Pearlman, J. D. et al. Magnetic resonance mapping demonstrates benefits of VEGF-induced myocardial angiogenesis. Nat Med 1, 1085-1089 (1995).

58 Kornowski, R. et al. Electromagnetic guidance for catheter-based transendocardial injection: a platform for intramyocardial angiogenesis therapy. Results in normal and ischemic porcine models. J Am Coll Cardiol 35, 1031-1039 (2000).

59 Ruixing, Y., Jiaquan, L., Jie, C. \& Dezhai, Y. Intravenous administration of vascular endothelial growth factor improves cardiac performance and inhibits cardiomyocyte apoptosis. Growth Factors 24, 209-217, doi:10.1080/08977190600760053 (2006).

60 Hao, X. et al. Myocardial angiogenesis after plasmid or adenoviral VEGFA(165) gene transfer in rat myocardial infarction model. Cardiovasc Res 73, 481-487, doi:10.1016/j.cardiores.2006.10.011 (2007).

61 Rosengart, T. K. et al. Angiogenesis gene therapy: phase I assessment of direct intramyocardial administration of an adenovirus vector expressing VEGF121 cDNA to individuals with clinically significant severe coronary artery disease. Circulation 100, 468-474 (1999).

62 Losordo, D. W. et al. Gene therapy for myocardial angiogenesis: initial clinical results with direct myocardial injection of phVEGF165 as sole therapy for myocardial ischemia. Circulation 98, 2800-2804 (1998).

63 Schalch, P. et al. Adenoviral-mediated transfer of vascular endothelial growth factor 121 cDNA enhances myocardial perfusion and exercise performance in the nonischemic state. J Thorac Cardiovasc Surg 127, 535-540, doi:10.1016/j.jtcvs.2003.06.015 (2004).

64 Dunlap, J. C. Molecular bases for circadian clocks. Cell 96, 271-290 (1999).

65 McLoughlin, S. C. \& FitzGerald, G. A. Between a rock and a hard place: how to align our circadian rhythms? Circulation 127, 19-20, 
doi:10.1161/CIRCULATIONAHA.112.150417 (2013).

66 Turek, F. W. et al. Obesity and metabolic syndrome in circadian Clock mutant mice. Science 308, 1043-1045, doi:10.1126/science.1108750 (2005).

67 Vitaterna, M. H. et al. Mutagenesis and mapping of a mouse gene, Clock, essential for circadian behavior. Science 264, 719-725 (1994).

68 Young, M. W. Circadian rhythms. Marking time for a kingdom. Science 288, 451-453 (2000).

69 Reppert, S. M. \& Weaver, D. R. Molecular analysis of mammalian circadian rhythms. Annu Rev Physiol 63, 647-676, doi:10.1146/annurev.physiol.63.1.647 (2001).

70 Hirayama, J. et al. Common light signaling pathways controlling DNA repair and circadian clock entrainment in zebrafish. Cell Cycle 8, 27942801 (2009).

71 Ripperger, J. A., Schmutz, I. \& Albrecht, U. PERsuading nuclear receptors to dance the circadian rhythm. Cell Cycle 9, 2515-2521 (2010).

72 Andretic, R. \& Hirsh, J. Circadian modulation of dopamine receptor responsiveness in Drosophila melanogaster. Proc Natl Acad Sci U S A 97, 1873-1878 (2000).

73 Millar-Craig, M. W., Bishop, C. N. \& Raftery, E. B. Circadian variation of blood-pressure. Lancet 1, 795-797 (1978).

74 Spengler, C. M., Czeisler, C. A. \& Shea, S. A. An endogenous circadian rhythm of respiratory control in humans. J Physiol 526 Pt 3, 683-694 (2000).

75 van den Buuse, M. Circadian rhythms of blood pressure, heart rate, and locomotor activity in spontaneously hypertensive rats as measured with radio-telemetry. Physiol Behav 55, 783-787 (1994).

76 Hastings, M. H. Neuroendocrine rhythms. Pharmacol Ther 50, 35-71 (1991).

77 Sahar, S. \& Sassone-Corsi, P. Circadian clock and breast cancer: a molecular link. Cell Cycle 6, 1329-1331 (2007).

78 Marcheva, B. et al. Disruption of the clock components CLOCK and BMAL1 leads to hypoinsulinaemia and diabetes. Nature 466, 627-631, doi:10.1038/nature09253 (2010).

79 Muller, J. E. et al. Circadian variation in the frequency of onset of acute myocardial infarction. $N$ Engl $J$ Med 313, 1315-1322, doi:10.1056/NEJM198511213132103 (1985).

80 Shea, S. A. Obesity and pharmacologic control of the body clock. $N$ Engl $J$ Med 367, 175-178, doi:10.1056/NEJMcibr1204644 (2012).

81 Kelly-Hayes, M. et al. Temporal patterns of stroke onset. The Framingham Study. Stroke 26, 1343-1347 (1995).

82 Knutsson, A. Health disorders of shift workers. Occup Med (Lond) 53, 103-108 (2003).

83 Drake, C. L., Roehrs, T., Richardson, G., Walsh, J. K. \& Roth, T. Shift work sleep disorder: prevalence and consequences beyond that of symptomatic day workers. Sleep 27, 1453-1462 (2004). 
84 Rouhi, P. et al. Pathological angiogenesis facilitates tumor cell dissemination and metastasis. Cell Cycle 9, 913-917 (2010).

85 Jensen, L. D. et al. Zebrafish models to study hypoxia-induced pathological angiogenesis in malignant and nonmalignant diseases. Birth Defects Res C Embryo Today 93, 182-193, doi:10.1002/bdrc.20203 (2011).

86 Carmeliet, P. Angiogenesis in health and disease. Nat Med 9, 653-660, doi:10.1038/nm0603-653 (2003).

87 Jensen, L. D., Cao, R. \& Cao, Y. In vivo angiogenesis and lymphangiogenesis models. Curr Mol Med 9, 982-991 (2009).

88 Sottile, J. Regulation of angiogenesis by extracellular matrix. Biochim Biophys Acta 1654, 13-22, doi:10.1016/j.bbcan.2003.07.002 (2004).

89 Senger, D. R. et al. Tumor cells secrete a vascular permeability factor that promotes accumulation of ascites fluid. Science 219, 983-985 (1983).

90 Ferrara, N. \& Henzel, W. J. Pituitary follicular cells secrete a novel heparin-binding growth factor specific for vascular endothelial cells. Biochem Biophys Res Commun 161, 851-858 (1989).

91 Shibuya, M. Vascular endothelial growth factor receptor-2: its unique signaling and specific ligand, VEGF-E. Cancer Sci 94, 751-756 (2003).

92 Suto, K., Yamazaki, Y., Morita, T. \& Mizuno, H. Crystal structures of novel vascular endothelial growth factors (VEGF) from snake venoms: insight into selective VEGF binding to kinase insert domain-containing receptor but not to fms-like tyrosine kinase-1. J Biol Chem 280, 21262131, doi:10.1074/jbc.M411395200 (2005).

93 Yamazaki, Y. et al. Snake venom Vascular Endothelial Growth Factors (VEGF-Fs) exclusively vary their structures and functions among species. J Biol Chem 284, 9885-9891, doi:10.1074/jbc.M809071200 (2009).

94 Cao, Y. Positive and negative modulation of angiogenesis by VEGFR1 ligands. Sci Signal 2, re1, doi:10.1126/scisignal.259re1 (2009).

95 Breier, G., Albrecht, U., Sterrer, S. \& Risau, W. Expression of vascular endothelial growth factor during embryonic angiogenesis and endothelial cell differentiation. Development 114, 521-532 (1992).

96 Ferrara, N. Molecular and biological properties of vascular endothelial growth factor. J Mol Med (Berl) 77, 527-543 (1999).

97 Neufeld, G., Cohen, T., Gengrinovitch, S. \& Poltorak, Z. Vascular endothelial growth factor (VEGF) and its receptors. FASEB $J \mathbf{1 3}, 9-22$ (1999).

98 Yla-Herttuala, S. \& Martin, J. F. Cardiovascular gene therapy. Lancet 355, 213-222, doi:10.1016/S0140-6736(99)04180-X (2000).

99 Ferrara, N. Role of vascular endothelial growth factor in regulation of physiological angiogenesis. Am J Physiol Cell Physiol 280, C1358-1366 (2001).

100 Brown, J. C., Sasaki, T., Gohring, W., Yamada, Y. \& Timpl, R. The Cterminal domain $\mathrm{V}$ of perlecan promotes betal integrin-mediated cell adhesion, binds heparin, nidogen and fibulin-2 and can be modified by glycosaminoglycans. Eur J Biochem 250, 39-46 (1997). 
101 Olofsson, B. et al. Vascular endothelial growth factor B, a novel growth factor for endothelial cells. Proc Natl Acad Sci U S A 93, 2576-2581 (1996).

102 Joukov, V. et al. A novel vascular endothelial growth factor, VEGF-C, is a ligand for the Flt4 (VEGFR-3) and KDR (VEGFR-2) receptor tyrosine kinases. EMBO J 15, 1751 (1996).

103 Lee, J. et al. Vascular endothelial growth factor-related protein: a ligand and specific activator of the tyrosine kinase receptor Flt4. Proc Natl Acad Sci US A 93, 1988-1992 (1996).

104 Kukk, E. et al. VEGF-C receptor binding and pattern of expression with VEGFR-3 suggests a role in lymphatic vascular development. Development 122, 3829-3837 (1996).

105 Lymboussaki, A., Olofsson, B., Eriksson, U. \& Alitalo, K. Vascular endothelial growth factor (VEGF) and VEGF-C show overlapping binding sites in embryonic endothelia and distinct sites in differentiated adult endothelia. Circ Res 85, 992-999 (1999).

106 Oh, S. J. et al. VEGF and VEGF-C: specific induction of angiogenesis and lymphangiogenesis in the differentiated avian chorioallantoic membrane. Dev Biol 188, 96-109, doi:10.1006/dbio.1997.8639 (1997).

107 Jeltsch, M. et al. Hyperplasia of lymphatic vessels in VEGF-C transgenic mice. Science 276, 1423-1425 (1997).

108 Kitadai, Y. et al. Clinicopathological significance of vascular endothelial growth factor (VEGF)-C in human esophageal squamous cell carcinomas. Int J Cancer 93, 662-666 (2001).

109 Hashimoto, I. et al. Vascular endothelial growth factor-C expression and its relationship to pelvic lymph node status in invasive cervical cancer. $\mathrm{Br}$ $J$ Cancer 85, 93-97, doi:10.1054/bjoc.2001.1846 (2001).

110 Cao, Y. et al. Vascular endothelial growth factor $\mathrm{C}$ induces angiogenesis in vivo. Proc Natl Acad Sci U S A 95, 14389-14394 (1998).

111 Achen, M. G. et al. Vascular endothelial growth factor D (VEGF-D) is a ligand for the tyrosine kinases VEGF receptor 2 (Flk1) and VEGF receptor 3 (Flt4). Proc Natl Acad Sci U S A 95, 548-553 (1998).

112 Stacker, S. A. et al. VEGF-D promotes the metastatic spread of tumor cells via the lymphatics. Nat Med 7, 186-191, doi:10.1038/84635 (2001).

113 Veikkola, T. \& Alitalo, K. VEGFs, receptors and angiogenesis. Semin Cancer Biol 9, 211-220, doi:10.1006/scbi.1998.0091 (1999).

114 Iyer, S. et al. The crystal structure of human placenta growth factor-1 (PlGF-1), an angiogenic protein, at 2.0 A resolution. $J$ Biol Chem 276, 12153-12161, doi:10.1074/jbc.M008055200 (2001).

115 DiPalma, T. et al. The placenta growth factor gene of the mouse. Mamm Genome 7, 6-12 (1996).

116 Takahashi, A. et al. Markedly increased amounts of messenger RNAs for vascular endothelial growth factor and placenta growth factor in renal cell carcinoma associated with angiogenesis. Cancer Res 54, 4233-4237 (1994).

117 De Falco, S., Gigante, B. \& Persico, M. G. Structure and function of 
placental growth factor. Trends Cardiovasc Med 12, 241-246 (2002).

118 Manning, G., Whyte, D. B., Martinez, R., Hunter, T. \& Sudarsanam, S. The protein kinase complement of the human genome. Science 298, 19121934, doi:10.1126/science.1075762 (2002).

119 Krause, D. S. \& Van Etten, R. A. Tyrosine kinases as targets for cancer therapy. N Engl J Med 353, 172-187, doi:10.1056/NEJMra044389 (2005).

120 Olsson, A. K., Dimberg, A., Kreuger, J. \& Claesson-Welsh, L. VEGF receptor signalling - in control of vascular function. Nat Rev Mol Cell Biol 7, 359-371, doi:10.1038/nrm1911 (2006).

121 Dixelius, J. et al. Ligand-induced vascular endothelial growth factor receptor-3 (VEGFR-3) heterodimerization with VEGFR-2 in primary lymphatic endothelial cells regulates tyrosine phosphorylation sites. J Biol Chem 278, 40973-40979, doi:10.1074/jbc.M304499200 (2003).

122 Seetharam, L. et al. A unique signal transduction from FLT tyrosine kinase, a receptor for vascular endothelial growth factor VEGF. Oncogene 10, 135-147 (1995).

123 Waltenberger, J., Claesson-Welsh, L., Siegbahn, A., Shibuya, M. \& Heldin, C. H. Different signal transduction properties of KDR and Flt1, two receptors for vascular endothelial growth factor. $J$ Biol Chem 269, 26988-26995 (1994).

124 Park, J. E., Chen, H. H., Winer, J., Houck, K. A. \& Ferrara, N. Placenta growth factor. Potentiation of vascular endothelial growth factor bioactivity, in vitro and in vivo, and high affinity binding to Flt-1 but not to Flk-1/KDR. J Biol Chem 269, 25646-25654 (1994).

125 Asahara, T. et al. VEGF contributes to postnatal neovascularization by mobilizing bone marrow-derived endothelial progenitor cells. EMBO J 18, 3964-3972, doi:10.1093/emboj/18.14.3964 (1999).

$126 \mathrm{Li}$, B. et al. Receptor-selective variants of human vascular endothelial growth factor. Generation and characterization. J Biol Chem 275, 2982329828, doi:10.1074/jbc.M002015200 (2000).

127 Gille, H. et al. Analysis of biological effects and signaling properties of Flt-1 (VEGFR-1) and KDR (VEGFR-2). A reassessment using novel receptor-specific vascular endothelial growth factor mutants. $J$ Biol Chem 276, 3222-3230, doi:10.1074/jbc.M002016200 (2001).

128 Larrivee, B. \& Karsan, A. Signaling pathways induced by vascular endothelial growth factor (review). Int J Mol Med 5, 447-456 (2000).

129 Kaipainen, A. et al. Expression of the fms-like tyrosine kinase 4 gene becomes restricted to lymphatic endothelium during development. Proc Natl Acad Sci U S A 92, 3566-3570 (1995).

130 Joukov, V. et al. Proteolytic processing regulates receptor specificity and activity of VEGF-C. EMBO $J$ 16, 3898-3911, doi:10.1093/emboj/16.13.3898 (1997).

131 Karkkainen, M. J., Makinen, T. \& Alitalo, K. Lymphatic endothelium: a new frontier of metastasis research. Nat Cell Biol 4, E2-5, doi:10.1038/ncb0102-e2 (2002).

132 Achen, M. G., McColl, B. K. \& Stacker, S. A. Focus on 
lymphangiogenesis in tumor metastasis. Cancer Cell 7, 121-127, doi:10.1016/j.ccr.2005.01.017 (2005).

133 Liotta, L. A. et al. Metastatic potential correlates with enzymatic degradation of basement membrane collagen. Nature 284, 67-68 (1980).

134 Corpechot, C. et al. Hypoxia-induced VEGF and collagen I expressions are associated with angiogenesis and fibrogenesis in experimental cirrhosis. Hepatology 35, 1010-1021, doi:10.1053/jhep.2002.32524 (2002).

135 Ke, Q. \& Costa, M. Hypoxia-inducible factor-1 (HIF-1). Mol Pharmacol 70, 1469-1480, doi:10.1124/mol.106.027029 (2006).

136 Semenza, G. L. Hypoxia-inducible factor 1: master regulator of $\mathrm{O} 2$ homeostasis. Curr Opin Genet Dev 8, 588-594 (1998).

137 Carmeliet, P. et al. Role of HIF-1alpha in hypoxia-mediated apoptosis, cell proliferation and tumour angiogenesis. Nature 394, 485-490, doi:10.1038/28867 (1998).

138 Rankin, E. B. \& Giaccia, A. J. The role of hypoxia-inducible factors in tumorigenesis. Cell Death Differ 15, 678-685, doi:10.1038/cdd.2008.21 (2008).

139 Semenza, G. L. \& Wang, G. L. A nuclear factor induced by hypoxia via de novo protein synthesis binds to the human erythropoietin gene enhancer at a site required for transcriptional activation. Mol Cell Biol 12, 5447-5454 (1992).

140 Chang, S. H., Worley, L. A., Onken, M. D. \& Harbour, J. W. Prognostic biomarkers in uveal melanoma: evidence for a stem cell-like phenotype associated with metastasis. Melanoma Res 18, 191-200, doi:10.1097/CMR.0b013e3283005270 (2008).

141 Lindner, V. et al. Members of the Jagged/Notch gene families are expressed in injured arteries and regulate cell phenotype via alterations in cell matrix and cell-cell interaction. Am $J$ Pathol 159, 875-883, doi:10.1016/S0002-9440(10)61763-4 (2001).

142 Semenza, G. L. Hydroxylation of HIF-1: oxygen sensing at the molecular level. Physiology (Bethesda) 19, 176-182, doi:10.1152/physiol.00001.2004 (2004).

143 Bilton, R. L. \& Booker, G. W. The subtle side to hypoxia inducible factor (HIFalpha) regulation. Eur J Biochem 270, 791-798 (2003).

144 Maxwell, P. H. et al. Hypoxia-inducible factor-1 modulates gene expression in solid tumors and influences both angiogenesis and tumor growth. Proc Natl Acad Sci U S A 94, 8104-8109 (1997).

145 Kaelin, W. G. Proline hydroxylation and gene expression. Annu Rev Biochem 74, 115-128, doi:10.1146/annurev.biochem.74.082803.133142 (2005).

146 Conway, E. M., Collen, D. \& Carmeliet, P. Molecular mechanisms of blood vessel growth. Cardiovasc Res 49, 507-521 (2001).

147 Liu, L. X. et al. Stabilization of vascular endothelial growth factor mRNA by hypoxia-inducible factor 1. Biochem Biophys Res Commun 291, 908914, doi:10.1006/bbrc.2002.6551 (2002). 
148 Lai, E. C. Notch signaling: control of cell communication and cell fate. Development 131, 965-973, doi:10.1242/dev.01074 (2004).

149 Artavanis-Tsakonas, S., Rand, M. D. \& Lake, R. J. Notch signaling: cell fate control and signal integration in development. Science 284, 770-776 (1999).

150 Rebay, I., Fehon, R. G. \& Artavanis-Tsakonas, S. Specific truncations of Drosophila Notch define dominant activated and dominant negative forms of the receptor. Cell 74, 319-329 (1993).

151 Villa, N. et al. Vascular expression of Notch pathway receptors and ligands is restricted to arterial vessels. Mech Dev 108, 161-164 (2001).

152 Roca, C. \& Adams, R. H. Regulation of vascular morphogenesis by Notch signaling. Genes Dev 21, 2511-2524, doi:10.1101/gad.1589207 (2007).

153 De Strooper, B. et al. A presenilin-1-dependent gamma-secretase-like protease mediates release of Notch intracellular domain. Nature 398, 518522, doi:10.1038/19083 (1999).

154 Weinmaster, G. Notch signaling: direct or what? Curr Opin Genet Dev 8, 436-442 (1998).

155 Davis, R. L. \& Turner, D. L. Vertebrate hairy and Enhancer of split related proteins: transcriptional repressors regulating cellular differentiation and embryonic patterning. Oncogene 20, 8342-8357, doi:10.1038/sj.onc.1205094 (2001).

156 Nakagawa, O. et al. Members of the HRT family of basic helix-loop-helix proteins act as transcriptional repressors downstream of Notch signaling. Proc Natl Acad Sci U S A 97, 13655-13660, doi:10.1073/pnas.250485597 (2000).

157 Shawber, C. J. et al. Notch alters VEGF responsiveness in human and murine endothelial cells by direct regulation of VEGFR-3 expression. $J$ Clin Invest 117, 3369-3382, doi:10.1172/JCI24311 (2007).

158 Kato, H. et al. Involvement of RBP-J in biological functions of mouse Notch1 and its derivatives. Development 124, 4133-4141 (1997).

159 Krebs, L. T. et al. Haploinsufficient lethality and formation of arteriovenous malformations in Notch pathway mutants. Genes Dev 18, 2469-2473, doi:10.1101/gad.1239204 (2004).

160 Shutter, J. R. et al. Dll4, a novel Notch ligand expressed in arterial endothelium. Genes Dev 14, 1313-1318 (2000).

161 Mailhos, C. et al. Delta4, an endothelial specific notch ligand expressed at sites of physiological and tumor angiogenesis. Differentiation 69, 135144, doi:10.1046/j.1432-0436.2001.690207.x (2001).

162 Segarra, M. et al. Dll4 activation of Notch signaling reduces tumor vascularity and inhibits tumor growth. Blood 112, 1904-1911, doi:10.1182/blood-2007-11-126045 (2008).

$163 \mathrm{Li}$, J. L. et al. Delta-like 4 Notch ligand regulates tumor angiogenesis, improves tumor vascular function, and promotes tumor growth in vivo. Cancer Res 67, 11244-11253, doi:10.1158/0008-5472.CAN-07-0969 (2007).

164 Krebs, L. T. et al. Notch signaling is essential for vascular morphogenesis 
in mice. Genes Dev 14, 1343-1352 (2000).

165 Claxton, S. \& Fruttiger, M. Periodic Delta-like 4 expression in developing retinal arteries. Gene Expr Patterns 5, 123-127, doi:10.1016/j.modgep.2004.05.004 (2004).

166 Diez, H. et al. Hypoxia-mediated activation of Dll4-Notch-Hey2 signaling in endothelial progenitor cells and adoption of arterial cell fate. Exp Cell Res 313, 1-9, doi:10.1016/j.yexcr.2006.09.009 (2007).

167 Gustafsson, M. V. et al. Hypoxia requires notch signaling to maintain the undifferentiated cell state. Dev Cell 9, 617-628, doi:10.1016/j.devcel.2005.09.010 (2005).

168 Gerhardt, H. et al. VEGF guides angiogenic sprouting utilizing endothelial tip cell filopodia. J Cell Biol 161, 1163-1177, doi:10.1083/jcb.200302047 (2003).

169 Li, J. L. \& Harris, A. L. Crosstalk of VEGF and Notch pathways in tumour angiogenesis: therapeutic implications. Front Biosci (Landmark Ed) 14, 3094-3110 (2009).

170 Cao, Y. Endogenous angiogenesis inhibitors and their therapeutic implications. Int J Biochem Cell Biol 33, 357-369 (2001).

171 Chen, P. H., Chen, X. \& He, X. Platelet-derived growth factors and their receptors: structural and functional perspectives. Biochim Biophys Acta 1834, 2176-2186, doi:10.1016/j.bbapap.2012.10.015 (2013).

172 Krupinski, J. et al. A putative role for platelet-derived growth factor in angiogenesis and neuroprotection after ischemic stroke in humans. Stroke 28, 564-573 (1997).

173 Cao, R. et al. Angiogenesis stimulated by PDGF-CC, a novel member in the PDGF family, involves activation of PDGFR-alphaalpha and alphabeta receptors. FASEB $J$ 16, 1575-1583, doi:10.1096/fj.02-0319com (2002).

174 Pinzani, M. \& Macias-Barragan, J. Update on the pathophysiology of liver fibrosis. Expert Rev Gastroenterol Hepatol 4, 459-472, doi:10.1586/egh.10.47 (2010).

175 Burgess, W. H. \& Maciag, T. The heparin-binding (fibroblast) growth factor family of proteins. Annu Rev Biochem 58, 575-606, doi:10.1146/annurev.bi.58.070189.003043 (1989).

176 Beenken, A. \& Mohammadi, M. The FGF family: biology, pathophysiology and therapy. Nat Rev Drug Discov 8, 235-253, doi:10.1038/nrd2792 (2009).

177 Itoh, N. \& Ornitz, D. M. Evolution of the Fgf and Fgfr gene families. Trends Genet 20, 563-569, doi:10.1016/j.tig.2004.08.007 (2004).

178 Haub, O. \& Goldfarb, M. Expression of the fibroblast growth factor-5 gene in the mouse embryo. Development 112, 397-406 (1991).

179 Johnson, D. E. \& Williams, L. T. Structural and functional diversity in the FGF receptor multigene family. Adv Cancer Res 60, 1-41 (1993).

180 Cao, R. et al. Collaborative interplay between FGF-2 and VEGF-C promotes lymphangiogenesis and metastasis. Proc Natl Acad Sci U S A 109, 15894-15899, doi:10.1073/pnas.1208324109 (2012). 
181 Nissen, L. J. et al. Angiogenic factors FGF2 and PDGF-BB synergistically promote murine tumor neovascularization and metastasis. $J$ Clin Invest 117, 2766-2777, doi:10.1172/JCI32479 (2007).

182 Cao, R. et al. Angiogenic synergism, vascular stability and improvement of hind-limb ischemia by a combination of PDGF-BB and FGF-2. Nat Med 9, 604-613, doi:10.1038/nm848 (2003).

183 Cao, Y., Cao, R. \& Hedlund, E. M. R Regulation of tumor angiogenesis and metastasis by FGF and PDGF signaling pathways. $J$ Mol Med (Berl) 86, 785-789, doi:10.1007/s00109-008-0337-z (2008).

184 Gupta, M. K. \& Qin, R. Y. Mechanism and its regulation of tumor-induced angiogenesis. World J Gastroenterol 9, 1144-1155 (2003).

185 Liekens, S., De Clercq, E. \& Neyts, J. Angiogenesis: regulators and clinical applications. Biochem Pharmacol 61, 253-270 (2001).

186 Tandle, A., Blazer, D. G., 3rd \& Libutti, S. K. Antiangiogenic gene therapy of cancer: recent developments. $J$ Transl Med 2, 22, doi:10.1186/1479-5876-2-22 (2004).

187 O'Reilly, M. S. et al. Angiostatin: a novel angiogenesis inhibitor that mediates the suppression of metastases by a Lewis lung carcinoma. Cell 79, 315-328 (1994).

188 Moser, T. L. et al. Endothelial cell surface F1-F0 ATP synthase is active in ATP synthesis and is inhibited by angiostatin. Proc Natl Acad Sci U S A 98, 6656-6661, doi:10.1073/pnas.131067798 (2001).

189 O'Reilly, M. S. et al. Endostatin: an endogenous inhibitor of angiogenesis and tumor growth. Cell 88, 277-285 (1997).

190 Sudhakar, A. et al. Human tumstatin and human endostatin exhibit distinct antiangiogenic activities mediated by alpha v beta 3 and alpha 5 beta 1 integrins. Proc Natl Acad Sci $U$ S A 100, 4766-4771, doi:10.1073/pnas.0730882100 (2003).

191 Dhanabal, M. et al. Endostatin induces endothelial cell apoptosis. J Biol Chem 274, 11721-11726 (1999).

192 Abdollahi, A. et al. Endostatin's antiangiogenic signaling network. Mol Cell 13, 649-663 (2004).

193 Ferrara, N., Hillan, K. J., Gerber, H. P. \& Novotny, W. Discovery and development of bevacizumab, an anti-VEGF antibody for treating cancer. Nat Rev Drug Discov 3, 391-400, doi:10.1038/nrd1381 (2004).

194 Gerber, H. P. \& Ferrara, N. Pharmacology and pharmacodynamics of bevacizumab as monotherapy or in combination with cytotoxic therapy in preclinical studies. Cancer Res 65, 671-680 (2005).

195 Kim, K. J. et al. Inhibition of vascular endothelial growth factor-induced angiogenesis suppresses tumour growth in vivo. Nature 362, 841-844, doi:10.1038/362841a0 (1993).

196 Quesada, A. R., Munoz-Chapuli, R. \& Medina, M. A. Anti-angiogenic drugs: from bench to clinical trials. Med Res Rev 26, 483-530, doi:10.1002/med.20059 (2006).

197 Motzer, R. J. et al. Activity of SU11248, a multitargeted inhibitor of vascular endothelial growth factor receptor and platelet-derived growth 
factor receptor, in patients with metastatic renal cell carcinoma. $J$ Clin Oncol 24, 16-24, doi:10.1200/JCO.2005.02.2574 (2006).

198 Faivre, S. et al. Safety, pharmacokinetic, and antitumor activity of SU11248, a novel oral multitarget tyrosine kinase inhibitor, in patients with cancer. J Clin Oncol 24, 25-35, doi:10.1200/JCO.2005.02.2194 (2006).

199 Holash, J. et al. VEGF-Trap: a VEGF blocker with potent antitumor effects. Proc Natl Acad Sci $U$ S A 99, 11393-11398, doi:10.1073/pnas.172398299 (2002).

200 Forero, A. et al. Phase I study of 90Y-CC49 monoclonal antibody therapy in patients with advanced non-small cell lung cancer: effect of chelating agents and paclitaxel co-administration. Cancer Biother Radiopharm 20, 467-478, doi:10.1089/cbr.2005.20.467 (2005).

201 Rugo, H. S. et al. Phase I trial of the oral antiangiogenesis agent AG013736 in patients with advanced solid tumors: pharmacokinetic and clinical results. $J$ Clin Oncol 23, 5474-5483, doi:10.1200/JCO.2005.04.192 (2005).

202 Streisinger, G., Walker, C., Dower, N., Knauber, D. \& Singer, F. Production of clones of homozygous diploid zebra fish (Brachydanio rerio). Nature 291, 293-296 (1981).

203 Haffter, P. et al. The identification of genes with unique and essential functions in the development of the zebrafish, Danio rerio. Development 123, 1-36 (1996).

204 Driever, W. et al. A genetic screen for mutations affecting embryogenesis in zebrafish. Development 123, 37-46 (1996).

205 Bosworth, C. A. t., Chou, C. W., Cole, R. B. \& Rees, B. B. Protein expression patterns in zebrafish skeletal muscle: initial characterization and the effects of hypoxic exposure. Proteomics 5, 1362-1371, doi:10.1002/pmic.200401002 (2005).

206 Marques, I. J. et al. Transcriptome analysis of the response to chronic constant hypoxia in zebrafish hearts. J Comp Physiol B 178, 77-92, doi:10.1007/s00360-007-0201-4 (2008).

207 Cao, Y. Monotherapy versus combination therapy of angiogenic and arteriogenic factors for the treatment of ischemic disorders. Curr Mol Med 9, 967-972 (2009).

208 Makino, Y. et al. Inhibitory PAS domain protein is a negative regulator of hypoxia-inducible gene expression. Nature 414, 550-554, doi:10.1038/35107085 (2001).

209 Cao, Y. Opinion: emerging mechanisms of tumour lymphangiogenesis and lymphatic metastasis. Nat Rev Cancer 5, 735-743, doi:10.1038/nrc1693 (2005).

210 Miklos, G. L. Bevacizumab in neoadjuvant treatment for breast cancer. $N$ Engl J Med 366, 1638; author reply 1639-1640, doi:10.1056/NEJMc1202229\#SA3 (2012).

211 Mountzios, G. \& Pentheroudakis, G. Bevacizumab in ovarian cancer. $N$ Engl J Med 366, 1257; author reply 1258, 
doi:10.1056/NEJMc1201044\#SA3 (2012).

212 Sculier, J. P., Meert, A. P. \& Paesmans, M. Bevacizumab for non-smallcell lung cancer. N Engl J Med 356, 1373-1374; author reply 1374-1375 (2007).

213 Sharieff, W. Bevacizumab in colorectal cancer. $N$ Engl J Med 351, 16901691; author reply 1690-1691 (2004).

214 Sonpavde, G. Bevacizumab in renal-cell cancer. N Engl J Med 349, 1674, doi:10.1056/NEJM200310233491719 (2003).

215 Bramwell, V. H. Pazopanib and the treatment palette for soft-tissue sarcoma. Lancet 379, 1854-1856, doi:10.1016/S0140-6736(12)60739-9 (2012).

216 des Guetz, G., Uzzan, B., Chouahnia, K. \& Morere, J. F. Cardiovascular toxicity of anti-angiogenic drugs. Target Oncol 6, 197-202, doi:10.1007/s11523-011-0204-7 (2011).

217 Randall, L. M. \& Monk, B. J. Bevacizumab toxicities and their management in ovarian cancer. Gynecol Oncol 117, 497-504, doi:10.1016/j.ygyno.2010.02.021 (2010).

218 Sato, Y. et al. Properties of two VEGF receptors, Flt-1 and KDR, in signal transduction. Ann N Y Acad Sci 902, 201-205; discussion 205-207 (2000).

219 Kamba, T. et al. VEGF-dependent plasticity of fenestrated capillaries in the normal adult microvasculature. Am J Physiol Heart Circ Physiol 290, H560-576, doi:10.1152/ajpheart.00133.2005 (2006).

220 Launay-Vacher, V. \& Deray, G. Hypertension and proteinuria: a classeffect of antiangiogenic therapies. Anticancer Drugs 20, 81-82, doi:10.1097/CAD.0b013e3283161012 (2009).

221 Perren, T. J. et al. A phase 3 trial of bevacizumab in ovarian cancer. $N$ Engl J Med 365, 2484-2496, doi:10.1056/NEJMoa1 103799 (2011).

222 Wang, J. et al. Novel mechanism of macrophage-mediated metastasis revealed in a zebrafish model of tumor development. Cancer Res 75, 306315, doi:10.1158/0008-5472.CAN-14-2819 (2015). 

PAPER 



\title{
Hypoxia-induced retinopathy model in adult zebrafish
}

\author{
Ziquan Cao ${ }^{1,4}$, Lasse D Jensen ${ }^{2,4}$, Pegah Rouhi ${ }^{2,4}$, Kayoko Hosaka ${ }^{2}$, Toste Länne ${ }^{1}$, John F Steffensen ${ }^{3}$, \\ Erik Wahlberg ${ }^{1} \&$ Yihai Cao $^{2}$
}

'Institute of Medicine and Health, Linkoping University, Linkoping, Sweden. ${ }^{2}$ Department of Microbiology, Tumor and Cell Biology, Karolinska Institutet, Stockholm, Sweden. ${ }^{3}$ Marine Biological Laboratory, Biological Institute, University of Copenhagen, Helsingor, Denmark. ${ }^{4}$ These authors contributed equally to this work. Correspondence should be addressed to Y.C. (yihai.cao@ki.se).

\section{Published online 4 November 2010; doi:10.1038/nprot.2010.149}

Hypoxia-induced vascular responses, including angiogenesis, vascular remodeling and vascular leakage, significantly contribute to the onset, development and progression of retinopathy. However, until recently there were no appropriate animal disease models recapitulating adult retinopathy available. In this article, we describe protocols that create hypoxia-induced retinopathy in adult zebrafish. Adult fli1:EGFP zebrafish are placed in hypoxic water for 3-10 d and retinal neovascularization is analyzed using confocal microscopy. It usually takes $11 \mathrm{~d}$ to obtain conclusive results using the hypoxia-induced retinopathy model in adult zebrafish. This model provides a unique opportunity to study kinetically the development of retinopathy in adult animals using noninvasive protocols and to assess therapeutic efficacy of orally active antiangiogenic drugs.

\section{INTRODUCTION}

Vascular pathology and metabolic alterations often lead to tissue hypoxia, which triggers several host responses including vasodilation, elevated hematopoiesis and angiogenesis ${ }^{1-3}$. In type 2 diabetes, retinopathy as a common complication usually results from hypoxiainduced neovascularization due to abnormal metabolism ${ }^{4}$. However, development of a clinically relevant and noninvasive hypoxia-induced retinopathy model in animals is a challenging task, which hampers assessment of the efficacy of potential therapeutic drugs.

To study the impact of hypoxia-induced pathological angiogenesis on retinopathy development, we have recently developed novel methods of hypoxia-induced retinopathy in adult zebrafish ${ }^{5}$. As in vivo angiogenesis-dependent disease models, zebrafish have several advantages over other existing disease models in mammals ${ }^{6-8}$. These include availability of genetically manipulated zebrafish strains, functional knockdown of specific genes by the morpholino technology, easy and low-cost maintenance of the zebrafish facility, and the capacity to breed a large number of zebrafish within a relatively short period of time. Using adult fli1:EGFP zebrafish ${ }^{9}$, we developed a hypoxia-induced retinopathy model in which retinal neovascularization occurs at the predicted region of the optic disc ${ }^{5}$. In this model, adult zebrafish are placed in a specifically designed aquarium and water oxygen levels can be automatically monitored using an oxygen sensor. After exposure to hypoxic water for a few days, retinal neovascularization is readily detected under a fluorescent microscope.

During exposure to hypoxia, it is convenient to add orally active, small chemical compounds to the hypoxic water so the antiangiogenic activity can be detected relative to controls ${ }^{5}$. As the retinal vasculature in the adult zebrafish consists of a monolayer network of microvessels that are composed of highly organized arterioles, capillaries and venules, hypoxia-induced vessel growth in different regions can be easily quantified by simply counting the number of sprouts, new branches and vascularization areas.

In contrast to our zebrafish model, other existing mammalian retinopathy models ${ }^{10}$ use invasive procedures such as laser-induced damage to trigger retinal neovascularization ${ }^{10}$. However, these invasive procedures are far from being clinically relevant, because retinopathy-associated pathology in clinical settings rarely involves mechanical damage other than metabolically or immunologically linked tissue hypoxia. In this regard, our current protocol is highly relevant to the clinical situation as compared with other existing models. This hypoxia-induced retinopathy model in adult zebrafish allows researchers to study the mechanisms of hypoxia-induced angiogenesis in vivo; to study the vessel formation, vascular remodeling, and leakiness under pathological settings; to correlate vascular changes with disease development; to screen potential therapeutic agents that are orally active; and to assess the beneficial effects of known antiangiogenic agents for the treatment of retinopathy. It should be emphasized that it is often inconvenient to expose adult mammals to a hypoxic chamber to induce retinopathy. Moreover, unlike the existing mammalian retinopathy models, detection of retinopathy in zebrafish does not require immunohistochemical staining, owing to the availability of fli1:EGFP and flk1:EGFP strains ${ }^{11}$. Our protocols do not require sophisticated facilities, and skillful experimenters should be able to implement this method in their research.

\section{Advantages and limitations}

Key advantages:

-It is inexpensive to obtain a large number of adult zebrafish.

- Maintenance of zebrafish is simple and convenient.

- Generation of genetic strains by deletion or overexpression of specific genes can be easily accomplished using available tools.

- Genetically manipulated zebrafish strains that are defective or have acquired functions of certain gene products are available.

- Genetic strains that express reporter genes in particular cell types are also available.

- Addition of orally active chemical stimulators or inhibitors to the aquarium water enables analysis of intervention of these compounds on physiological and pathological processes.

- Hypoxia setups for zebrafish are simple and can be tightly controlled. Oxygen levels in the fish aquarium can be accurately adjusted using an automated monitor and control system.

- The turnover time for experiments is relatively short.

Limitations:

- It is difficult to obtain local tissue or organ hypoxia rather than systemic exposure to hypoxia.

- Owing to the limitation of available antibodies against zebrafish proteins, it is difficult to perform immunohistochemical analysis using zebrafish tissues. 


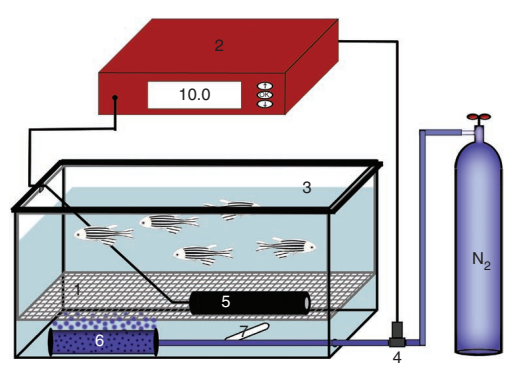

Figure 1 | Hypoxia setup for adult zebrafish. (1) Breeding insert net; (2) oxygen regulator; (3) adult hypoxia aquarium; (4) solenoid valve; (5) oxygen electrode; (6) air stone; and (7) magnetic bar. Electrical wires are marked as black lines. The compressed nitrogen source is shown as a blue tank, and the nitrogen-containing gas tubes are marked with blue.

- Owing to the small size of zebrafish, skillful and careful operations are required to perform experiments.

\section{Experimental design}

Creation of hypoxic conditions. Creation of a hypoxic aquarium for zebrafish might be achieved by either perfusing nitrogen gas into water directly or into the air inside a sealed aquarium. However, perfusion of nitrogen gas into the aquarium air may create high pressure and lead to high death rates in zebrafish. In our experimental design, we chose to perfuse nitrogen gas into the water of the aquarium (see EQUIPMENT SETUP for further information). As shown in Figure 1, a 1-liter aquarium was chosen for convenience and for reducing the cost of drug treatment. Smaller aquaria may present difficulties of accurately adjusting oxygen levels in the $\mathrm{H}_{2} \mathrm{O}$. The aquarium is designed as an automated unit, which automatically controls nitrogen gas perfusion depending on oxygen levels in the water. Oxygen levels are accurately measured by an oxygen electrode connected to an oxygen regulator and the nitrogen gas supplier. Despite the automation of the hypoxic aquarium, pilot studies should be conducted before experimentation by placing a few zebrafish into the aquarium for at least $3 \mathrm{~d}$. In case of unexpected early death of zebrafish during experimentation, the oxygen regulator should be recalibrated.

Replicates and controls. Owing to the variation of individual zebrafish in response to hypoxia, a relatively large number of zebrafish should be included in each experimental group. To obtain statistically meaningful results, typically 12 adult zebrafish should be used in each experiment; at least two experiments are recommended in order to repeat the findings. To design each experiment optimally, all necessary controls should be included. The age, sex and physical status of all zebrafish under hypoxic and normoxic conditions should be identical. The normoxic aquarium, as a control, should be similarly designed as the hypoxic chamber except lacking nitrogen gas supply. Both aquaria should be placed in the same environment, as temperature, moisture and oxygen content in the room may affect experimental results.

Drug treatment. When designing drug treatment experiments, chemical solvents used as vehicles are added to control groups under normoxic and hypoxic conditions. Thus, for each experiment testing the effects of a drug on hypoxia-induced retinal neovascularization, there are three controls: vehicle in normoxia, drug in normoxia and vehicle in hypoxia. The least toxic vehicle for a particular drug should be chosen. If ethanol or DMSO is used as vehicle, we strongly recommend having sufficiently strong stock solutions so that the final concentration of the vehicle is less than $0.1 \%$ in water; this will minimize the unspecific effects of the vehicle. For example, in a previous study we used anti-VEGF drugs such as sunitinib to study the effects of VEGF on hypoxia-induced retinal neovascularization ${ }^{5}$. In that study, sunitinib was dissolved in citrate buffer at $8 \mathrm{mg} \mathrm{ml}^{-1}$ $(15 \mathrm{mM})$ and diluted 30,000 times to reach a final concentration of $0.5 \mu \mathrm{M}$ in the hypoxic water.

Results analysis. In the assessment of experimental findings, both left and right eyes of each zebrafish are used to validate results. However, variations of retinal vascularization between the two eyes of each zebrafish may exist. Dissection of zebrafish eyes and isolation of the retina are extremely challenging tasks and demand technically skillful micro-operations under a stereoscope. To ensure high success rates, it is strongly recommended that the investigator should practice on a daily basis and avoid intake of adenosine receptor activators such as coffee, which may increase uncontrolled hand movements. Once the retinal samples are prepared, we recommend examining the results as soon as possible as EGFP fluorescence undergoes progressive quenching. At the stage of results analysis, similar regions of hypoxia- and normoxia-exposed retinal vasculature should be used for comparative evaluation because the architecture and structure of retinal vessels vary in different regions (Figs. 2 and 3). It is important to quantify intercapillary distance, vessel density, and new branches and sprouts using the same magnification; preferably, 15-25 images from at least six individual fish from each experiment should be quantified to reach a justified conclusion.

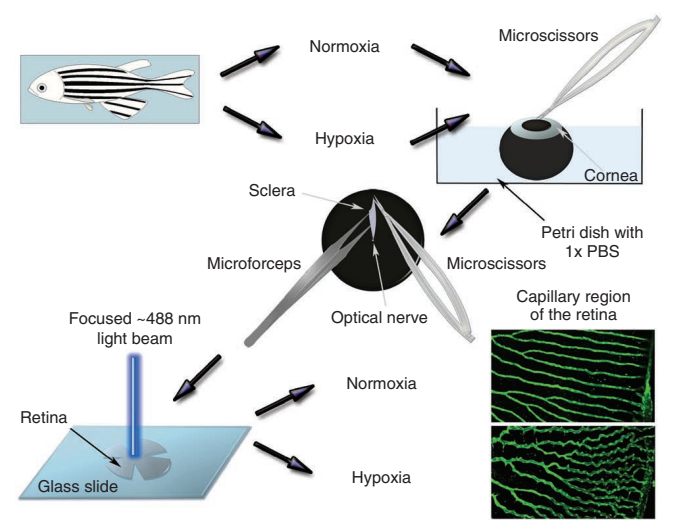

Figure 2 | Critical steps of dissecting and analyzing the retinal vasculature in adult zebrafish. Adult fli1:EGFP zebrafish exposed to normoxia and hypoxia were anesthetized with tricaine and killed. All the fish were immersed immediately in 4\% PFA for $24 \mathrm{~h}$. Fish eyes were enucleated and placed into a Petri dish containing $1 \times$ PBS. Using fine surgical forceps and scissors, fish retinas were carefully isolated and examined under a fluorescent microscope. 
Figure 3 | Hypoxia-induced retinal angiogenesis. (a-d) The retinal vasculature of adult zebrafish is divided into three regions: the arterial region (AR), which consists of I-IV grade of arteries ${ }^{5}$; the capillary area (CA); and the collecting veins (ColV). For the quantification of angiogenic responses, each region should be quantified separately. (a) High-magnification view of the area defined by a blue box in $\mathbf{b}$. (b) Confocal micrograph of EGFP-positive vessels in the retina of a zebrafish exposed to normoxic water.

(c) Confocal micrograph of EGFP-positive vessels in the retina of a zebrafish exposed to hypoxic water (10\% air saturation) for 6 d. (d) High magnification of the area defined by a blue box in c. Under the hypoxic conditions shown in our protocol, CA (between dashed red lines), spanning the $350 \mu \mathrm{m}$ between the arterial region and collecting veins, usually shows considerable angiogenic responses, whereas AR and ColV exhibit minor alterations. For quantification of intercapillary distance, a midline (purple line in $\mathbf{b}$ and c, at the position of $175 \mu \mathrm{m}$ from ColV) should be drawn and intercapillary distances are measured crossing this line, as shown in a and $\mathbf{d}$ (purple bidirectional arrows). Arrows point to sprouts and arrowheads indicate endothelial tips. Yellow dots in $\mathbf{b}$ and $\mathbf{c}$ indicate branch points. CV, circumferential vein. Scale bars in $\mathbf{a}$ and $\mathbf{d}, 25 \mu \mathrm{m}$. Scale bars in $\mathbf{b}$ and $\mathbf{c}, 100 \mu \mathrm{m}$.

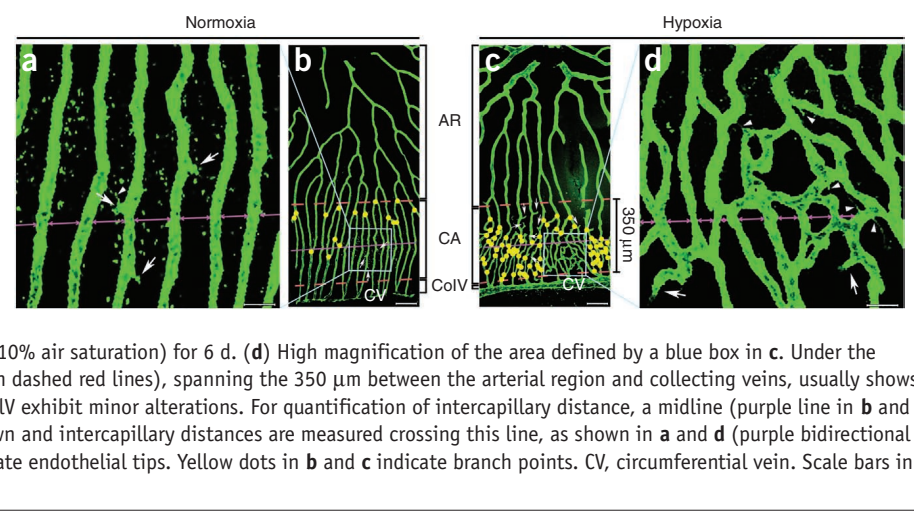

\section{MATERIALS}

\section{REAGENTS}

- $1 \times$ PBS (prepared in house or purchased from Hyclone, cat. no. SH30028.02)

- Colorless nail polish

- Compressed nitrogen (97\%; AGA Gas)

- Distilled water $\left(\mathrm{dH}_{2} \mathrm{O}\right)$

- Ethyl 3-aminobenzoate methanesulfonic acid salt (98\%, tricaine;

Sigma-Aldrich, cat. no. E10521)

- Paraformaldehyde (PFA, prills, 95\%; Sigma-Aldrich, cat. no. 441244-1KG)

! CAUTION Highly toxic. Small solid PFA particles or fumes can easily be inhaled during preparation or handling of the $4 \%$ solution. Always work with gloves and never open PFA-filled containers or tubes outside a fume hood.

- Vectashield mounting medium (Vector Laboratories, cat. no. H-1000)

- Zebrafish (Tg(fli1:EGFP) $)^{\mathrm{y} 1}, 3-24$ months old; ZFIN)

- Sodium hydroxde (NaOH; Sigma, cat. no. S5881)

- Hydrochloric acid (HCl; Merck, cat. no. 3.0317-1)

\section{EQUIPMENT}

- Adobe Photoshop CS3 (or later versions; Adobe)

- Aquatic oxygen monitor and regulator (made in house, see EQUIPMENT SETUP and further information on the following homepage: http://www. mbl.ku.dk/JFSteffensen/OxygenTemperatureMonitorRegulator/.

Alternatively, can be purchased from Loligo, cat. no. OX10000)

- Bacterial culture dish (10 cm; Corning, cat. no. 391-1517)

- BD falcon 50-ml polypropylene conical tubes (BD Biosciences, cat. no. 358206)

- BD Micro-Fine and 1-ml BD U-100 insulin syringe $(0.33 \mathrm{~mm}$;

BD Biosciences, cat. no. 305930)

- Confocal microscope (Nikon D-eclipse C1; Nikon)

- Confocal software (EZ-C1 3.9 Nikon digital eclipse; Nikon)

- Dissecting stereomicroscope (Wild M3Z; Leica)

- Eppendorf polypropylene conical tubes ( $2 \mathrm{ml}$; VWR International,

cat. no. 211-2120)

- Fish transfer nets (Fridhems Akvarier)

- Forceps superfine tips (Dumonts finest tip, AgnTho's, cat. no. 11254-20)

- Glass beaker (100 ml, Boro 3.3)

- Magnet bar (4 cm; VWR International, cat. no. 442-4527)

- Magnet stirrer with heating (VMS-A; VWR International, cat. no. 442-0185)

- Microscope coverslips (Thermo Scientific, cat. no. 631-0135)

- Microscope slides (Thermo Scientific, cat. no. 631-1303)

- Microsoft Office Excel 2003 (or later version; Microsoft)

- Nonheated magnet stirrer (VS-C7-2 EU-1; VWR International, cat. no. 442-0551)

- Plastic containers (185 mm ×85 mm ×75 mm; Aqua-Schwarz)

- Plastic lid for zebrafish hypoxia chamber $(200 \mathrm{~mm} \times 100 \mathrm{~mm} \times 14 \mathrm{~mm}$;

Aqua-Schwarz)

- Plastic pipette tips (10-1,000 $\mu$ l; Sarstedt, cat. no. 70762.100)

- Reusable adhesive (Sense; Clas Ohlson, cat. no. 26-888)

- Scalpel blades (no 11, Allgaier instrumente; AgnTho's AB, cat. no. 02-040-011)

- Scalpel holder (no. 3, Allgaier instrumente; AgnTho's AB, cat no. 02-030-030)

- Spatula/microspoon (VWR International, cat. no. 231-1354)
- Surgical scissors (2 and 6 mm, Vanna; AgnTho's AB, cat. no. 15000-03)

- Tissue needle, straight tip (VWR International, cat. no. 238-6250)

- Zebrafish spawn insert (175 mm $\times 80 \mathrm{~mm} \times 63 \mathrm{~mm}$; Aqua-Schwarz)

- Programmable LED indicator (PR Electronics, cat. no. 5714)

- Solenoid valve (Bürkert)

- OxyGuard mini oxygen electrode (OxyGuard, cat. no. D161SV)

- ImageJ software

\section{REAGENT SETUP}

4\% PFA (wt/vol) Dissolve $20 \mathrm{~g}$ PFA powder in $500 \mathrm{ml}$ of $1 \times$ PBS by warming the solution to $60^{\circ} \mathrm{C}$ under constant stirring or in a water bath for about $16 \mathrm{~h}$ The $4 \%$ PFA fixative solution can be kept at $4{ }^{\circ} \mathrm{C}$ for a maximum of 2 weeks.

$\Delta$ CRITICAL Fresh solution is crucial to the success of experiments. If PFA solution has been stored longer than 2 weeks, discard the old solution and prepare a fresh batch.

Tricaine stock $\mathbf{( 0 . 0 5 \% )}$ For convenience, a $25 \times$ stock solution in $\mathrm{d}_{2} \mathrm{O}$ $(0.05 \%)$ should be prepared. This stock solution is diluted to a final concentration $(0.002 \%)$ immediately before use. Tricaine powder $(50 \mathrm{mg})$ is dissolved in $100 \mathrm{ml} \mathrm{dH}_{2} \mathrm{O}$ to obtain the stock solution. Prepare 2-ml aliquots in plastic vials and store them at $-20^{\circ} \mathrm{C}$ until use. $\Delta$ CRITICAL Tricaine is unstable at low concentrations $(2 \times$ concentration or lower) in aqueous solutions. Do not prepare a stock solution at a low concentration.

\section{EQUIPMENT SETUP}

Hypoxia aquarium setup The aquarium is a standard 1-liter plastic chamber $(185 \mathrm{~mm} \times 85 \mathrm{~mm} \times 75 \mathrm{~mm})$ with a plastic lid containing a hole on one side to allow installation of the nitrogen supply. The hole is located at the bottom corner of the short side of the aquarium (Fig. 1). Insert a gas tube through the hole, which is sealed with plastic cement to prevent leakage. An air stone is connected to the end of the gas tube and is placed at the bottom on the opposite side of the aquarium. The other end of the tube is connected to the solenoid regulator valve that controls nitrogen perfusion from the compressed nitrogen bottle. Place a magnetic bar at a central position at the bottom of the aquarium, which is placed on a nonheated magnetic stirrer. Switch on the stirrer and rotate the magnetic bar gently. Create another hole $(2 \mathrm{~mm})$ on the opposite side of the aquarium; this hole should be located close to the lid. Through this hole, a wire connected to the oxygen electrode is inserted. Place a breeding insert (mesh size: $2 \mathrm{~mm}$ ) into the aquarium and leave the bottom several millimeters from the top of the air stone. The breeding insert prevents physical contact between zebrafish and the magnetic stirring bar. Place the oxygen electrode at the bottom of the breeding insert at a position such that the electrode tip is far from the air stone. Use plastic cement to seal the space between the wire and hole. Create a hole $(5 \mathrm{~mm}$ in diameter) in the lid; this hole releases the air pressure within the aquarium. Oxygen regulator setup The oxygen regulator is essentially a programmable LED indicator equipped with a solenoid valve from Bürkert and an OxyGuard mini oxygen electrode as described on the following website: http://www.mbl.ku.dk/JFSteffensen/OxygenTemperatureMonitorRegulator/. 
PROTOCOL

\section{PROCEDURE}

\section{Adaptation to hypoxic environment $\bigcirc$ TIMING $36 \mathrm{~h}$}

1) Add 0.9 liters of prewarmed water $\left(28.5^{\circ} \mathrm{C}\right)$ to a hypoxia aquarium and calibrate the system to display $100 \%$ air saturation in normoxic water and to show the measurement indicator at the zero position when short circuited (' 0 cal' on the bottom of the front panel of the oxygen regulator) (Fig. 1).

$\triangle$ CRITICAL STEP The water level in the aquarium should not exceed $80 \%$ of the total aquarium depth. Leave approximately $20 \%$ air space for controlling oxygen exchange.

2) (Optional) Dissolve orally active chemical compounds in a desirable vehicle such as DMSO, ethanol or water (or, in the case of sunitinib, citrate buffer) as a stock solution.

3| (Optional) Add the chemical compounds to the aquarium water at concentrations that functionally intervene with hypoxia-induced host responses. In the case of sunitinib, add $30 \mu \mathrm{l}$ of $8 \mathrm{mg} \mathrm{ml}^{-1}$ sunitinib stock solution to the 0.9 -liter aquarium water (from Step 1).

4| Transfer 10-12 healthy adult fli1:EGFP transgenic zebrafish (3 to 24 months old) to the normoxic water in the aquarium. Stir water at a relatively slow rotation speed to ensure sufficient circulation of water in the entire aquarium ( 150 r.p.m.).

$\Delta$ CRITICAL STEP Water temperature in the hypoxia aquarium should be equilibrated to the same temperature as the water in which the zebrafish are maintained. Larger magnetic bars are recommended for better mixing at slow rotation speeds.

$\triangle$ CRITICAL STEP Also place a group of adult zebrafish in normoxia (regular water) in an aquarium to serve as a control. If chemical compounds are being tested and have been added to the hypoxia aquarium at Step 3, add the appropriate amount of the same drug and vehicle to normoxia control aquaria (see Experimental design for further information on controls). Thus, three controls—-vehicle in normoxia, vehicle in hypoxia and drug in normoxia-should be included in each experiment.

5| Turn on the nitrogen gas (approximately $3 \mathrm{~atm}$ ) in the hypoxia aquarium and perfuse nitrogen gas through the air stone into the water. Set the limit to $20 \%$ and hysteresis to 0.2 on the oxygen regulator (Fig. 1 ).

$\Delta$ CRITICAL STEP The nitrogen air stone should be kept clean so that nitrogen gas is released evenly by forming small bubbles. The nitrogen pressure should not be too high to prevent suffocation of fish.

6| Keep the fish at $20 \%$ air saturation for $8-12 \mathrm{~h}$; follow this by decreasing air saturation to $15 \%$ as shown on the oxygen regulator.

7| Keep the fish at $15 \%$ air saturation for 5-6 h, then decrease the air saturation to $13 \%$ as shown on the oxygen regulator.

I CAUTION At this time point, almost all zebrafish should show signs of having hypoxia by exhibiting water-surface breathing and elevated respiration frequencies. As a result of hypoxia, some zebrafish may die. It is crucial to remove dead fish immediately from the aquarium to prevent infection in surviving fish.

8| Keep the fish at $13 \%$ air saturation for 5-6 h; follow this by decreasing air saturation to $11.5 \%$ shown on the oxygen regulator.

9| Maintain the fish at $11.5 \%$ air saturation for $5-6 \mathrm{~h}$, then decrease air saturation to $10 \%\left(820\right.$ p.p.b. at $\left.28.5^{\circ} \mathrm{C}\right)$ as shown on the oxygen regulator. This time point is considered day 0 for exposure to hypoxic water.

\section{? TROUBLESHOOTING}

\section{Hypoxia exposure period and zebrafish dissection $\bigcirc$ TIMING 3-12 d}

10| Inspect the zebrafish in the hypoxia aquarium several times per day and ensure that the nitrogen pressure is steadily maintained at $10 \%$ air saturation. Should any fish die, remove them immediately from the aquarium.

\section{? TROUBLESHOOTING}

11 At the end of the hypoxia exposure period (usually 6-12 d), transfer zebrafish using a net to $50 \mathrm{ml}$ of $0.004 \%$ tricaine and keep them in tricaine for at least 10 min after cessation of gill movements. Fix the dead fish by submerging 1-4 fish in $30 \mathrm{ml}$ of $4 \%$ PFA pre-equilibrated to $4^{\circ} \mathrm{C}$ in a 50 - $\mathrm{ml}$ Falcon tube and incubate at $4{ }^{\circ} \mathrm{C}$ for at least $24 \mathrm{~h}$. 
PROTOCOL

Figure 4 | Critical steps of dissecting and flatting the adult zebrafish retina. (1) Create a small hole at the circumferential side of the cornea by a sharp syringe needle. (2) Cut off the cornea along the limbal basis with a pair of surgical microscissors. (3) Extrude the lens with microsurgery forceps. (4) Insert a sharp syringe needle between the sclera and the retina and create a small hole. (5) Expand the space between the retina and sclera with a needle and a pair of microscissors. (6) Tear off the sclera and choroid with forceps. (7) Position the retina in the middle of a glass slide with the inside facing up. $(8,9)$ Cut the retina disc three or four times, mount it in Vectashield and cover with a glass cover slip.
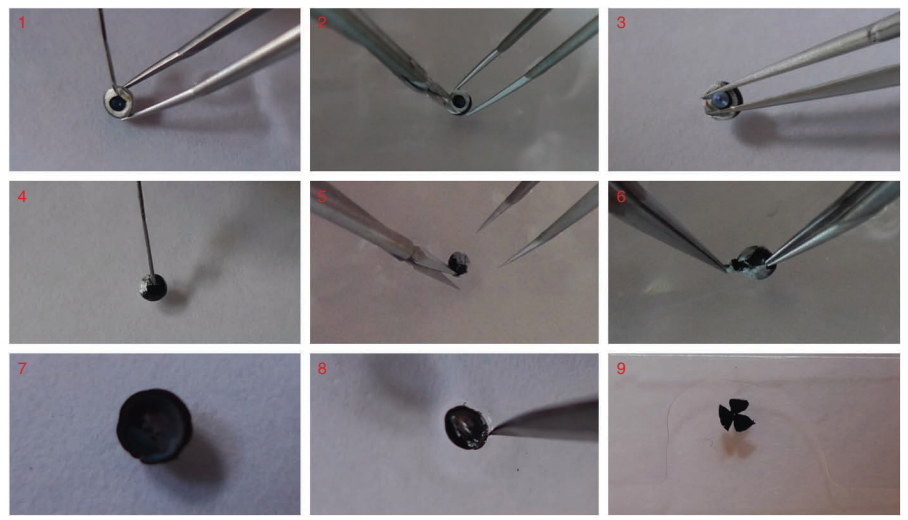

12| Wash the zebrafish with $1 \times$ PBS twice and transfer them to a Petri dish

(10 cm in diameter) containing $20 \mathrm{ml}$ of $1 \times$ PBS (Fig. 2). Dissociate fish heads from the rest of the body using a pair of fine surgical scissors. With scissors and forceps, gently disassociate the operculum and gills. The eyeballs connected to the optic nerve should now be apparent when inspecting the fish from the ventral side. Release the eyeballs by cutting the optic nerves as close to the eyeballs as possible.

PAUSE POINT Whole fish or fish eyes fixed in 4\% PFA can be stored in $1 \times$ PBS at $4{ }^{\circ} \mathrm{C}$ for up to 2 weeks before dissection and flat-mounting of the retinas.

\section{Zebrafish retina flat mounting $\bigcirc$ TIMING 15-60 min per retina}

13| In a plastic Petri dish (10 cm in diameter) containing $1 \times$ PBS, position the eye with the cornea upward using microsurgery forceps. Use a sharp syringe needle to create a wound at the circumferential side of the cornea. Carefully cut off the cornea along the limbal basis with a pair of surgical microscissors, then extrude the lens. Flip over the eye to place the optic nerve stump upward. Gently insert a sharp needle between the sclera (outer layer of the eye) and the retina and separate the two without damaging the retina. Expand the space between the retina and sclera with the needle and gently tear off the sclera and choroid (brown color) with forceps (Figs. 2 and 4 ).

14| Position the retina in the middle of a glass slide with the inside facing up. Carefully cut the retina disc three or four times from the edge toward the center to divide it evenly into three or four 'petals' of a flower-shaped sheet (Figs. 2 and 4). Remove excessive water with a piece of tissue paper and add a drop of Vectashield on the retina. Place a glass cover slide $(22 \mathrm{~mm} \times 22 \mathrm{~mm})$ on the retina and seal the edges with nail polish.

PAUSE POINT Mounted retinas can be stored horizontally in the dark at $-20^{\circ} \mathrm{C}$ for up to 2 weeks before microscopic examination.

I CAUTION From the time when zebrafish are killed, the fluorescent EGFP signal starts to degrade and quench. This degradation process can be delayed by fixation/mounting. For best results, we recommend microscopically analyzing eyes within 2 weeks after killing the zebrafish.

\section{Imaging TIMING 2-6 h}

15| To obtain an overview of a whole petal of the retina, which is often used to show the general changes in the retinal vasculature (for example, in presentations or publications), illuminate the sample at low magnification ( $\times 4$ objective) with blue light of approximately $488 \mathrm{~nm}$ and use the correct filter (i.e., EGFP or FITC) to obtain EGFP-positive signals using a fluorescent microscope. Alternatively, scan EGFP-positive signals at $488 \mathrm{~nm}$ using a confocal microscope (see EQUIPMENT). When using a confocal microscope at low magnification, the thickness of a scanned tissue layer in focus is high, indicating that the total number of scanned layers is relatively low (i.e., one or two). Therefore, image acquisition time is greatly shortened. For a more detailed image analysis, scan the samples at higher magnifications $(\times 10, \times 20, \times 40$ or $\times 63$ objectives $)$. At high magnification, images can reveal detailed information, including vascular branch points, sprouts, endothelial cell tips and filopodial extensions. The resolution should be at least 1,024 $\times 1,024$ (Figs. 2 and 3). For more information on imaging and quantification parameters, see Box 1. 


\section{BOX 1 | QUANTIFICATION OF RETINAL ANGIOGENESIS}

1. Analyze the vasculature of dissected retinas mounted on glass slides using confocal microscopy at $\times 4, \times 10$ or $\times 20$ magnification objectives (see Step 15).

2. Take serial micrographs of retinal vessels covering the vascular region from the central optic artery to the circumferential vein. Take at least 2-5 pictures from each retina, depending on the magnification. A calibrated scale bar indicating a fixed length (e.g., $100 \mu \mathrm{m}$ ) should be included in all images. Such a scale bar can be obtained by capturing a picture of a microscope ruler under the same microscope settings as used to capture images of the zebrafish retina. These serial micrographs of each retina allow definition of the arterial regions, capillary areas (anastomosis between arteries and collecting veins) and circumferential veins (Fig. 3). Hypoxia-induced vessel sprouts, branch formation, endothelial cell tips and networks can be detected in the capillary area (Fig. 3 ).

3. Define the capillary area to be used for quantification as an area spanning $350 \mu \mathrm{m}$ from the beginning of the collecting veins toward the arterial region (red dashed lines in Fig. 3). First rotate the image until the circumferential vein is perfectly horizontal. Next, if using Adobe Photoshop, define a fixed selection box with dimensions equal to the width of the image and a height of $350 \mu \mathrm{m}$, using the scale bar as a reference. Set the selection box at the beginning of the collecting veins (Fig. 3 ) and crop the selected area. Using ImageJ, you should first crop the image at the beginning of the collecting veins (Fig. 3 ), and then crop the image again such that its height is precisely $350 \mu \mathrm{m}$ (use the scale bar as a reference). The resulting cropped image should then have the same width and height $(350 \mu \mathrm{m})$ and is defined as the capillary area.

4. Draw a horizontal midline ( $175 \mu \mathrm{m}$ from both ends of the capillary area defined in the previous step) through the capillary area. In Adobe Photoshop, the line is drawn using the line tool, and the distance is measured using the calibrated size bar in each image as a reference. In ImageJ, the distance can be measured in the same way, and the image can be cropped at the midline rather than by drawing an actual line.

5. Measure intercapillary distance as shown in Figure $\mathbf{3}$ using the straight line selection tool followed by the measure tool in ImageJ. Measure all intercapillary distances crossing the midline from 15 to 25 images for each experimental condition and calculate the average.

6. With Adobe Photoshop, calculate the capillary density by selecting greens (or if the image is black and white, select highlights) using the color range tool. The number of selected pixels as seen from the histogram window is noted. This value is divided by the total number of pixels in the area, and multiplied by $100 \%$ to give the capillary density in percent. Calculate the average of 15-25 images per group.

7. Manually count all branching points in the capillary area of each micrograph shown as yellow dots in Figure 3. Calculate the average of $15-25$ images per group.

8. New vascular sprouts are defined as blunted vascular branches that have not connected to other vessels (Fig. 3, arrows). Manually count all sprouts in the capillary area of each micrograph. Calculate the average of 15-25 images per group.

9. Endothelial cell tips are defined as thin fibers, as shown in Figure $\mathbf{3}$ (arrowheads). Manually count endothelial cell tips in the capillary area of each micrograph. Calculate the average of 15-25 images per group.

10. Either Adobe Photoshop or ImageJ (which is free, open-source software) can be used for analysis. Although quantification procedures in ImageJ of vessel sprouts, branch formation and endothelial tips are exactly the same as described above using Adobe Photoshop, measurement of the vascular area is achieved by converting green fluorescent signals into white signals on a black background using the 'color-split channels' command in ImageJ followed by the selection of only the green channel. Use the 'binary' command followed by the 'analyze particles' command to measure the capillary area. The sum of the area of all particles is divided by the total number of pixels in the image and multiplied by $100 \%$ to give the capillary area in percent. Calculate the average of 15-25 images per group.

$\triangle$ CRITICAL STEP The retina is a relatively thick tissue and contains autofluorescent cells. Images acquired with fluorescent microscopy may therefore contain a relatively high, noisy background. We recommend that readers analyze the samples using confocal microscopy, if available.

\section{? TROUBLESHOOTING}

16| Analyze the data using the Adobe Photoshop software program. Vessel density, capillary vascular area, intercapillary distance and vascular branches can be accurately quantified as detailed in Box 1 (Figs. 2 and 3 ).

? TROUBLESHOOTING

\section{? TROUBLESHOOTING}

Troubleshooting advice can be found in Table 1. 
PROTOCOL

TABLE 1 | Troubleshooting table.

\begin{tabular}{|c|c|c|c|}
\hline Step & Problem & Possible reason & Solution \\
\hline \multirow[t]{5}{*}{9,10} & $\begin{array}{l}\text { All zebrafish die } \\
\text { after just a few days }\end{array}$ & $\begin{array}{l}\text { The oxygen level may } \\
\text { be too low }\end{array}$ & $\begin{array}{l}\text { Calibrate the oxygen electrode to make sure it shows an accurate } \\
\text { oxygen level. Have nitrogen flow at a rate where overshooting is } \\
\text { at a minimum. It is critical to watch the breathing rate of each } \\
\text { fish rather than simply monitoring the oxygen readers }\end{array}$ \\
\hline & & $\begin{array}{l}\text { The water may be } \\
\text { contaminated }\end{array}$ & $\begin{array}{l}\text { Make sure the water is of high quality (free from pollutants, } \\
\text { and with optimal conductivity and } \mathrm{pH} \text { ). Water quality can be } \\
\text { maintained relatively well throughout the experiment by not } \\
\text { feeding fish the day before and during the experiment, as well } \\
\text { as by removing dead fish immediately }\end{array}$ \\
\hline & & $\begin{array}{l}\text { The fish are exhausted } \\
\text { from swimming }\end{array}$ & $\begin{array}{l}\text { Make sure the rotation speed of the magnet is not too high for } \\
\text { fish that are forced to swim along the water current }\end{array}$ \\
\hline & & $\begin{array}{l}\text { The fish are fighting with } \\
\text { each other }\end{array}$ & $\begin{array}{l}\text { Have a sufficient number of fish in the tank from the beginning } \\
\text { (not fewer than six) and remove fish that are strongly } \\
\text { dominating the others }\end{array}$ \\
\hline & & The lid is sealed too tightly & $\begin{array}{l}\text { High air pressure can kill fish. Make sure the ventilation hole is } \\
\text { not blocked }\end{array}$ \\
\hline \multirow[t]{3}{*}{15,16} & $\begin{array}{l}\text { Lack of angiogenic } \\
\text { response }\end{array}$ & $\begin{array}{l}\text { The oxygen level is not } \\
\text { sufficiently low }\end{array}$ & $\begin{array}{l}\text { Calibrate the oxygen electrode to make sure it shows the } \\
\text { true oxygen level. Lower the oxygen level to } 8.5-9.0 \% \text { air } \\
\text { saturation. Carefully monitor fish behaviors such as breathing } \\
\text { rates and surface breathing }\end{array}$ \\
\hline & & Insufficient exposure time & Increase hypoxia exposure time \\
\hline & & Samples are stored too long & Analyze freshly prepared samples \\
\hline
\end{tabular}

\section{TIMING}

Steps 1-9, Adaptation to hypoxic environment: $36 \mathrm{~h}$

Steps 10-12, Hypoxia exposure period and zebrafish dissection: 3-12 d

Steps 13 and 14, Zebrafish retina flat mounting: 15-60 min per retina

Steps 15 and 16, Imaging: 2-6 h

\section{ANTICIPATED RESULTS}

According to the detailed experimental procedure, described in sequential steps above, the expected result of hypoxia-induced angiogenesis in adult fli1:EGFP zebrafish is shown in Figures 2 and $\mathbf{3}$. Under normoxia, the architecture of retinal vasculature is highly organized as a single layer of vascular network in which the optic artery is located at the center of the optic disc. About 4-9 arteries branch from the optic artery and are evenly distributed in each optic disc; these secondary arteries are further segregated 4-5 times before anastomosing with protrusions from the circumferential vein, which is located around the edge of the optic disc. The arterial-venous anastomosis area consists of microcapillaries that form double-ended capillary plexuses. Under hypoxia, subtle changes in the arteries, capillaries and circumferential veins can be easily detected by fluorescent microscopy. Kinetic studies show that capillary sprouts begin on day 3 and the formation of a vascular network becomes obvious on day 6 after exposure to hypoxia ${ }^{5}$. After day 6, hypoxia-induced endothelial tip and capillary branch formation are only present in the capillary area (Figs. 2 and 3), which can be easily quantified as numbers of branches and sprouts, intercapillary distance and vascular area. However, prolonged exposure to hypoxia after day 6 results in modest changes in the retinal vasculature ${ }^{5}$. As distribution of the optic vasculature in each zebrafish varies, at least six retinas should be used for assessing results. Typically, 4-9 grade I arterial branches from the central optic artery can be found in the zebrafish retina ${ }^{5,12}$. However, the number of sub-branches (grade II-IV) from grade I vessels may vary considerably depending on the number of the grade I vessels. In general, fewer grade I vessels would result in more branching grade II-IV vessels to cover the retinal disc. Therefore, for quantification, it is highly recommended to choose the same region of the optic disc for comparison (Box 1), and six retinas should be used. For confocal imaging analysis, we recommend mounting the isolated retina samples onto glass slides with cover slips using $\times 4, \times 10, \times 20$ or $\times 40$ magnification objectives. Figure 3 provides an example of hypoxia-induced retinal angiogenesis captured with the $\times 10$ magnification objective. 
PROTOCOL

ACKNOWLEDGMENTS This study was supported through research grants from the Swedish Research Council, the Swedish Cancer Foundation, the Karolinska Institute Foundation, the Karolinska Institute Distinguished Professor Award, the European Union Integrated Project of Metoxia (Project no. 222741) and the European Research Council (ERC) advanced grant ANGIOFAT (Project no 250021). Z.C. was supported by the Swedish Heart and Lung Foundation.

AUTHOR CONTRIBUTIONS Y.C. designed the study. L.D.J., P.R. and Z.C. performed the experiments. L.D.J., P.R., Z.C. and Y.C. analyzed the data. K.H., T.L., J.F.S. and E.W. participated in designing and discussing this study. L.D.J., P.R., Z.C. and Y.C. wrote the article.

\section{COMPETING FINANCIAL INTERESTS The authors declare no competing}

financial interests.

Published online at http://www.natureprotocols.com/.

Reprints and permissions information is available online at http://npg.nature. com/reprintsandpermissions/.

1. Boutin, A.T. et al. Epidermal sensing of oxygen is essential for systemic hypoxic response. Cell 133, 223-234 (2008).

2. Cao, Y. Monotherapy versus combination therapy of angiogenic and arteriogenic factors for the treatment of ischemic disorders. Curr. Mol. Med. 9, 967-972 (2009).
3. Makino, Y. et al. Inhibitory PAS domain protein is a negative regulator of hypoxia-inducible gene expression. Nature 414, 550-554 (2001).

4. Kondo, T. et al. Knockout of insulin and IGF-1 receptors on vascular endothelial cells protects against retinal neovascularization. J. Clin. Invest. 111, 1835-1842 (2003)

5. Cao, R., Jensen, L.D., Soll, I., Hauptmann, G. \& Cao, Y. Hypoxia-induced retinal angiogenesis in zebrafish as a model to study retinopathy. PLoS One 3, e2748 (2008).

6. Lee, S.L. et al. Hypoxia-induced pathological angiogenesis mediates tumor cell dissemination, invasion, and metastasis in a zebrafish tumor model. Proc. Natl Acad. Sci. USA 106, 19485-19490 (2009).

7. Rouhi, P. et al. Pathological angiogenesis facilitates tumor cell dissemination and metastasis. Cell Cycle 9, 913-917 (2010).

8. Dahl Ejby Jensen, L. et al. Nitric oxide permits hypoxia-induced lymphatic perfusion by controlling arterial-lymphatic conduits in zebrafish and glass catfish. Proc. Natl Acad. Sci. USA 106, 18408-18413 (2009)

9. Lawson, N.D. \& Weinstein, B.M. In vivo imaging of embryonic vascular development using transgenic zebrafish. Dev. Biol. 248, 307-318 (2002).

10. Gariano, R.F. \& Gardner, T.W. Retinal angiogenesis in development and disease. Nature 438, 960-966 (2005)

11. Fouquet, B., Weinstein, B.M., Serluca, F.C. \& Fishman, M.C. Vessel patterning in the embryo of the zebrafish: guidance by notochord. Dev. Biol. 183, 37-48 (1997).

12. Alvarez, Y. et al. Genetic determinants of hyaloid and retinal vasculature in zebrafish. BMC Dev. Biol. 7, 114 (2007) 
PAPER 



\title{
Hypoxia-induced metastasis model in embryonic zebrafish
}

\author{
Pegah Rouhi ${ }^{1,4}$, Lasse D Jensen ${ }^{1,4}$, Ziquan Cao $^{2,4}$, Kayoko Hosaka ${ }^{1}$, Toste Länne ${ }^{2}$, Eric Wahlberg ${ }^{2}$, \\ John Fleng Steffensen ${ }^{3} \&$ Yihai Cao ${ }^{1}$
}

\begin{abstract}
${ }^{1}$ Department of Microbiology, Tumor and Cell Biology, Karolinska Institutet, Stockholm, Sweden. ${ }^{2}$ Institute of Medicine and Health, Linkoping University, Linkoping, Sweden. ${ }^{3}$ Marine Biological Laboratory, Biological Institute, University of Copenhagen, Helsingor, Denmark. ${ }^{4}$ These authors contributed equally to this work. Correspondence should be addressed to Y.C. (yihai.cao@ki.se).

Published online 4 November 2010; doi:10.1038/nprot.2010.150

Hypoxia facilitates tumor invasion and metastasis by promoting neovascularization and co-option of tumor cells in the peritumoral vasculature, leading to dissemination of tumor cells into the circulation. However, until recently, animal models and imaging technology did not enable monitoring of the early events of tumor cell invasion and dissemination in living animals. We recently developed a zebrafish metastasis model to dissect the detailed events of hypoxia-induced tumor cell invasion and metastasis in association with angiogenesis at the single-cell level. In this model, fluorescent DiI-labeled human or mouse tumor cells are implanted into the perivitelline cavity of 48-h-old zebrafish embryos, which are subsequently placed in hypoxic water for $3 \mathrm{~d}$. Tumor cell invasion, metastasis and pathological angiogenesis are detected under fluorescent microscopy in the living fish. The average experimental time for this model is $7 \mathrm{~d}$. Our protocol offers a remarkable opportunity to study molecular mechanisms of hypoxia-induced cancer metastasis.
\end{abstract}

\section{INTRODUCTION}

Invasion and metastasis are the hallmarks of malignant disease, and understanding the molecular mechanisms underlying cancer metastasis may define new therapeutic targets for anticancer drug development. Hypoxia is known to significantly facilitate tumor invasion and metastasis in association with angiogenesis ${ }^{1}$. However, mechanisms of hypoxia-induced tumor invasion and metastasis remain poorly understood. One of the initial steps of the metastatic cascade involves the dissemination of malignant cells from the primary site ${ }^{2}$. Tumor cell dissemination in cancer patients can occur at the early stage of tumor development, when primary tumors are relatively small ${ }^{3}$. Unfortunately, current cancer detection methods at both clinical and preclinical levels are not able to image early events of tumor cell dissemination from primary sites.

Owing to the transparent and immunoprivileged nature of zebrafish embryos, we have recently developed a tumor invasion and metastasis model in association with hypoxia-induced angiogenesis ${ }^{2,4}$. This metastatic model allows us to detect tumor cell dissemination at the single-cell level. Using this method, we demonstrated that VEGF- or hypoxia-induced angiogenesis facilitate tumor cell dissemination ${ }^{2,4}$. Implantation of fluorescently labeled mouse or human tumor cells into the perivitelline space of zebrafish embryos, which are subsequently placed in a hypoxic aquarium, allows us to study early events of metastasis while primary tumors are relatively small. This is the first animal model to study detailed events of cancer metastasis in relation to tumor hypoxia and angiogenesis. This protocol is complementary to other existing models that are primarily designed to study the later events of the metastatic cascade, including the formation of metastatic niches and the regrowth of tumors in distal tissues and organs ${ }^{2,5,6}$. Unlike other models, monitoring of tumor cell dissemination with respect to angiogenesis and hypoxia in embryonic zebrafish can be achieved in living animals, which allows kinetic analysis of tumor cell dissemination and formation of distal metastatic niches in the same animal.
The general advantages of using zebrafish to study pathological angiogenesis and disease development are described by Cao et al. in this issue $\mathrm{e}^{7}$. Additionally, hypoxia-induced metastasis in zebrafish embryos provides an outstanding opportunity to define signaling components in the host, which facilitates tumor invasion, dissemination and metastasis using specific morpholino intervention technology. It should be emphasized that genetic manipulations of crucial components involving the hypoxia-induced host responses could also create global hypoxia in the host. For example, deletion of the von Hippel-Lindau tumor suppressor gene in zebrafish results in a systemic hypoxic response which recapitulates the key aspects of Chuvash polycythemia in humans ${ }^{8}$. These genetically altered hypoxic zebrafish models offer alternative options for studying the hypoxic effect on tumor angiogenesis and metastasis in zebrafish.

This protocol has broad applications for studying the mechanisms underlying cancer cell metastasis, invasion and spreading; for defining new molecular targets controlling tumor cell migration and hypoxia-regulated angiogenesis; for assessing antimetastatic effects of known anticancer drugs; and for discovering potential new drugs for the treatment of metastatic diseases. In addition to its use in the assessment of therapeutic efficacies of monotherapies, it is anticipated to be a powerful model to study the therapeutic effects of combination therapy containing two or more therapeutic molecules. This zebrafish metastatic model is complementary to existing mouse tumor and metastatic models, allowing the study of different aspects of cancer metastasis.

\section{Advantages and limitations}

Key advantages:

In addition to the general advantages and limitations of using zebrafish as an in vivo model to study hypoxia-induced angiogenesis (see the companion article by Cao et al. ${ }^{7}$ ), there are several other advantages of using zebrafish embryos for studying metastasis. These include: 
- Effective knockdown of gene expression by morpholino technology in zebrafish embryos allows readers to study functions of specific gene products.

- The transparent nature of zebrafish embryos allows visualization of vascular changes and tumor cell dissemination under hypoxia and normoxia in living fish.

- Immunoprivilege of zebrafish embryos allows implantation of mammalian tumor cells, including human and mouse tumor cells ${ }^{4}$. Limitations:

- As the hypoxia chamber is designed for the whole embryo, it is difficult to create hypoxia only in the tumor tissue.

- As most mammalian tumors grow at $37^{\circ} \mathrm{C}$, it is difficult to study the process of xenograft tumor growth at the optimal temperature.

- Microinjection of tumor cells into the perivitelline space of a large number of zebrafish embryos is a tedious procedure and requires highly skillful micro-operations.

\section{Experimental design}

Aquaria. The general setup and design for the hypoxic and normoxic aquaria are described in the companion article in this issue ${ }^{7}$. For zebrafish embryos, we recommend using a nylon mesh (mesh size $<0.25 \mathrm{~mm}$ ) to separate zebrafish embryos from the magnetic stirring bar, as fish embryos are unable to swim against water currents.

Tumor cells. Monolayers of human, mouse or other mammalian tumor cells should grow in culture to subconfluency (about 70-80\%) to ensure that they are at the proliferating phase. Although genetically manipulated tumor cells that express fluorescein-activated proteins such as red fluorescent protein or yellow fluorescent protein may be used for in vivo tracing, we do not recommend using enhanced green fluorescent protein (EGFP)-labeled tumor cells if fli1:EGFP or flk-1:EGFP zebrafish strains ${ }^{9,10}$ are used. For convenience, we use 1,1'-dioctadecyl-3,3,3',3'-tetramethylindocarbocyanine perchlorate (DiI), which labels cell membranes for a relatively long time ( $\sim 2$ weeks) without losing color ${ }^{4}$.

Embryo production. While growing tumor cells, at least 5-6 pairs of adult zebrafish of breeding age should be placed in the breeding chamber (one pair per chamber) to ensure production of 200-300 eggs. We highly recommend choosing as many healthy embryos produced by the same pair of adult zebrafish as possible, as they are usually at the identical age.

Tumor cell injection. Approximately 100 DiI-labeled tumor cells should be injected into the perivitelline space and should not be implanted into the yolk sac. As considerable variation exists between individual zebrafish embryos in response to hypoxia, a relatively large number of zebrafish embryos should be included in each experimental group; in each experimental and control group, 100 zebrafish embryos should be implanted with tumor cells. Implantation of tumor cells into the perivitelline space of embryos at $48 \mathrm{~h}$ post fertilization (h.p.f.) is technically challenging; thus, a skillful and experienced person should perform injections. The investigator should not drink coffee or other caffeine-enriched beverages in order to avoid uncontrolled hand movement.

Embryo selection. Even after tumor cell implantation, fish embryos should undergo further selection under fluorescent microscopy to ensure that tumor cells are located only within the perivitelline space.

Experimental conditions. To obtain statistically meaningful results, at least 50 embryos should be used in each experimental and control group, as it is expected that some embryos will die during experimentation. Once placed in the hypoxic chamber, we do not recommend opening the lid and disturbing embryos until they are harvested for analysis. For analysis, each individual zebrafish embryo in both hypoxic and normoxic groups should be photographed using fluorescent microscopy. These data should be digitally deposited in Adobe Photoshop, ImageJ or an equivalent program for further analysis.

Drug treatment. In drug treatment experiments, chemical solvent is used as vehicle and should have as low toxicity as possible. Dissolve the orally active chemical compound such as sunitinib in a desirable solution such as distilled water in high concentrations to prepare the stock solution. According to the appropriate final concentration of the drug in the hypoxia and normoxia aquaria, add the appropriate volume of the stock solution. As an example, if the stock concentration is $10 \mathrm{mM}$, to reach a final concentration of $1 \mu \mathrm{M}$ in 1 liter of hypoxic or normoxic aquaria embryo water, the drug should be diluted 10,000×; accordingly, $100 \mu$ l of stock should be added to the water.

Controls. In all experimental designs, appropriate controls should be used under normoxia and hypoxia. It is known that tumor cells, even though derived from the same origin of the same cell type, may have different potentials to trigger angiogenic responses and to metastasize. Thus, if applicable, tumor cells with high or low metastatic potentials from the same type of cancer cell should be considered to serve as positive and negative controls. If genetically manipulated tumor cells are used, it is essential to include two control groups under normoxia and hypoxia: (i) nonmanipulated wild-type tumor cells; and (ii) vector or mock-manipulated tumor cells.

\section{MATERIALS}

\section{REAGENTS}

- 1,1'-Dioctadecyl-3,3,3', $3^{\prime}$-tetramethylindocarbocyanine perchlorate

(DiI, Invitrogen, cat. no. D3899)

- 1-Phenyl-2-thiourea (PTU; Sigma-Aldrich, cat. no. P7629)

- Agarose (SeaKem LE, cat. no. 50004)

- Calcium chloride $\left(\mathrm{CaCl}_{2}\right.$, Sigma-Aldrich, cat. no. C8106)

- Distilled water $\left(\mathrm{dH}_{2} \mathrm{O}\right)$

- Dulbecco Modified Eagle's Medium (DMEM (Sigma-Aldrich, cat. no. D6546)

- Dulbecco's phosphate-buffered saline (DPBS, Sigma-Aldrich, cat. no. D8537)
- Ethyl 3-aminobenzoate methanesulfonic acid salt ((98\% purity) tricaine;

Sigma-Aldrich, cat. no. E10521)

- FBS (Hyclone, Thermo scientific, cat. no. SV30160.03)

- HEPES buffer (1 M in water; Sigma-Aldrich, cat. no. 83264)

- L-glutamine solution (200 mM, Gibco, cat. no. G7513)

- Magnesium sulfate $\left(\mathrm{MgSO}_{4}\right.$, Sigma-Aldrich, cat. no. M7506)

- Nail polish, colorless

- Nitrogen gas (100\%, AGA GAS AB)

- Penicillin-streptomycin solution (Gibco, cat. no. 15070-063)

- Potassium chloride (KCl; Sigma-Aldrich, cat. no. P5405)

- Sodium chloride (NaCl; Merck, cat. no. 1.06404.1000) 
- T241 mouse fibrosarcoma cell line (ATCC)

- Transgenic (fli1:EGFP) zebrafish were obtained from ZFIN (Zebrafish Model Organism Database; http://www.zfin.org/)

- Trypsin solution (2.5\% (wt/vol), 10×, Gibco, cat. no. 15090-046)

- Vectashield mounting medium (Vector Laboratories, cat. no. H-1000)

\section{EQUIPMENT}

- Photoshop CS3 or later versions (Adobe)

- ImageJ software

- Bacterial culture dish (10 cm; Corning, cat. no. 391-1517)

- BD Falcon polypropylene conical tubes ( $50 \mathrm{ml}$; BD Biosciences,

cat. no. 358206)

- $\mathrm{CO}_{2}$ incubator (Thermo electron corporation, model 311)

- Confocal microscope (Nikon D-eclipse C1, Nikon)

- Confocal software (EZ-C1 3.9 Nikon digital eclipse, Nikon)

- Costar cell culture plates (48-well; Corning, cat. no. 3548)

- Dissecting stereomicroscope (Wild M3Z, Leica)

- Eppendorf microinjector (FemtoJet 5247, Eppendorf,

cat. no. 5247000.013

- Eppendorf polypropylene conical tubes ( $2 \mathrm{ml}$, VWR International,

cat. no. 211-2120)

- Fish transfer nets (Fridhems Akvarier)

- Fluorescent software (NIS-Elements D 3.0, Nikon)

- Forceps superfine tips (Dumont's finest tip, AgnTho's AB, cat. no. 11254-20)

- Heraeus BK 800 refrigerated incubator (Thermo Scientific, cat. no. 50120350)

- Magnet bar (4 cm; VWR International, cat. no. 442-4527)

- Magnet stirrer with heating (VMS-A, VWR International,

cat. no. 442-0185

- Manipulator (MM33-Right, Märzhäuser Wetzlar,

cat. no. 00-42-101-0000)

- Microloader tips (0.5-10 $\mu$ l; Eppendorf, VWR, cat. no. 5242 965.003)

- Microscope cover slips (Thermo Scientific, cat. no. 631-0135)

- Microscope slides (Thermo Scientific, cat. no. 631-1303)

- Microsoft Office Excel 2003 or later version (Microsoft)

- Nonfilamentous borosilicate glass capillaries needles $(1.0 \mathrm{~mm}$ in diameter;

World Precision Instruments, cat. no. 1B100-4)

- Nonheating magnet stirrer (VS-C7-2 EU-1, VWR International,

cat. no. 442-0551)

- Oxygen regulator (OxyReg, Loligo, cat. no. OX10000)

- P-97 Flaming/Brown-type micropipette puller (Automated Scientific,

cat. no. SU-P-97)

- Pasteur pipette (150 mm, wide, Bergman Labora AB)

- Pipette pump (10 ml, Pi-pump, VWR, cat. no. 612-3756)

- Plastic containers $(185 \mathrm{~mm} \times 85 \mathrm{~mm} \times 75 \mathrm{~mm}$ and $200 \mathrm{~mm} \times 100 \mathrm{~mm} \times$

$130 \mathrm{~mm}$, Aqua-Schwarz)

- Plastic lid for zebrafish embryo hypoxia chamber $(200 \mathrm{~mm} \times 100 \mathrm{~mm} \times$

$14 \mathrm{~mm}$, and $245 \mathrm{~mm} \times 145 \mathrm{~mm}$, Aqua-Schwarz)

- Plastic pipette tips (10-1,000 $\mu$ l; Sarstedt, cat. no. 70762.100)

- Reusable adhesive (Sense, Clas Ohlson, cat. no. 26-888)

- Pipette (1 ml; Sarstedt, cat. no. 861251.001)

- Tissue needle (straight tip, VWR, cat. no. 238-6250)

-Zebrafish spawn insert $(175 \mathrm{~mm} \times 80 \mathrm{~mm} \times 63 \mathrm{~mm}$, Aqua-Schwarz

REAGENT SETUP

Tricaine stock $(0.05 \%(\mathbf{w t} / \mathrm{vol}))$ For convenience, a $25 \times$ stock solution in $\mathrm{dH}_{2} \mathrm{O}$ $(0.05 \%$ (wt/vol) ) should be prepared. To prepare the $25 \times$ stock, tricaine powder

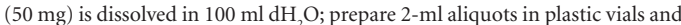
store at $-20^{\circ} \mathrm{C}$ until use. This stock solution is diluted to a final concentration $(2 \times, 0.004 \%(\mathrm{wt} / \mathrm{vol}))$ immediately before use. $\Delta$ CRITICAL Tricaine is unstable

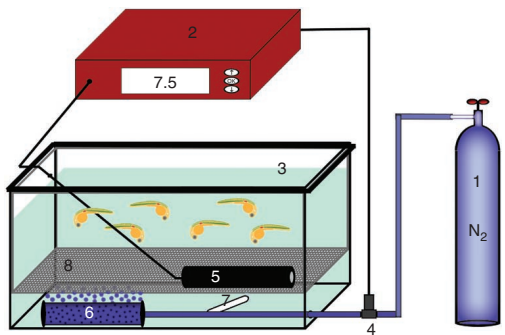

Figure 1 | Hypoxic setup for the embryonic zebrafish aquarium. (1) Nitrogen gas tank; (2) oxygen regulator; (3) embryonic hypoxia aquarium; (4) valves; (5) oxygen electrode; (6) air stone; (7) magnetic bar; and (8) nylon mesh.

at low concentrations $(2 \times$ concentration or lower $)$ in aqueous solutions. Do not make a stock solution at a low concentration.

Danieu's aquarium water $(30 \times)$

Prepare 1 liter sterile $\mathrm{dH}_{2} \mathrm{O}$ containing $1.74 \mathrm{M} \mathrm{NaCl}, 21 \mathrm{mM} \mathrm{KCl}, 12 \mathrm{mM}$ $\mathrm{MgSO}_{4}, 18 \mathrm{mM} \mathrm{CaCl}_{2}$ and $150 \mathrm{mM}$ HEPES buffer. This inorganic aquarium water can be kept at $22{ }^{\circ} \mathrm{C}$ for up to 12 months.

1-Phenyl-2-thiourea (PTU, 10 $\times$ ) Dissolve PTU powder in $\mathrm{dH}_{2} \mathrm{O}$ to a final concentration of $2 \mathrm{mM}$. $\Delta$ CRITICAL It usually takes several hours to dissolve PTU at room temperature with aid of a magnet stirrer. Once completely dissolved, PTU solution can be kept at $4{ }^{\circ} \mathrm{C}$ for a maximum of 2 weeks. Agarose gel preparation To prepare a solution containing $2 \%(\mathrm{wt} / \mathrm{vol})$ agarose, add $0.8 \mathrm{~g}$ agarose to $40 \mathrm{ml}$ distilled water in a glass beaker and heat the solution in a microwave oven until all agarose particles are dissolved. Add $20 \mathrm{ml}$ of the agarose solution into a Petri dish $(10 \mathrm{~cm}$ diameter $)$ and let it harden. About 15-20 min later, warm the rest of the solution in a microwave oven and add the solution on the top gel layer. Before solidification, place a mold in the center of the dish and leave it embedded in the agarose gel while it hardens. About $30 \mathrm{~min}$ later, remove the mold and add $20 \mathrm{ml}$ of $1 \%$ (vol/vol) sterile Danieu's aquarium water to immerse the gel. The agarose gel plate can be stored at $4{ }^{\circ} \mathrm{C}$ for $\sim 30 \mathrm{~d}$ until use. Agarose plates should be pre-equilibrated to $26-28^{\circ} \mathrm{C}$ before microinjection.

Preparation of DiI stock solution According to the manufacturer's instructions, add $97 \%$ (vol/vol) ethanol to the powder compound to constitute a concentration of $20 \mathrm{mg} \mathrm{ml}^{-1}$; mix the solution to ensure that the DiI compound is completely dissolved. This stock solution can be stored in $5-\mu \mathrm{l}$ aliquots at $-20^{\circ} \mathrm{C}$.

DMEM cell culture medium preparation (FBS-DMEM) To prepare this medium, supplement the DMEM with $10 \%$ (vol/vol) FBS. Also add $1 \%$ (vol/vol) L-glutamine and $1 \%(\mathrm{vol} / \mathrm{vol})$ penicillin-streptomycin. Store this medium at $4{ }^{\circ} \mathrm{C}$. Before use, it should be pre-equilibrated to room temperature or, preferably, to $37^{\circ} \mathrm{C}$.

\section{EQUIPMENT SETUP}

Hypoxia aquarium for zebrafish embryos A hypoxia chamber for zebrafish embryos is created using a 1-liter standard plastic aquarium with a lid (Fig. 1). In general, the hypoxia chamber setup for zebrafish embryos is the same as for adult zebrafish (see Cao et al. ${ }^{7}$ ), with the exception that the bottom of the insert chamber is made of fine nylon mesh (mesh size $<0.25 \mathrm{~mm}$ ).

\section{PROCEDURE}

\section{Tumor cell injection in zebrafish embryos TIMING $\sim 3 \mathrm{~d}$}

1| Day 0: place a breeding insert inside a 1 liter breeding aquarium, fill the aquarium with aquarium water and put one male and one female adult fli1:EGFP zebrafish inside the breeding insert. Let the zebrafish mate overnight; fertilized eggs can be harvested the following morning.

2| Day 1: the next morning, collect eggs from fertilized fli1:EGFP-transgenic zebrafish and incubate them at $28.5^{\circ} \mathrm{C}$ in the Danieu's solution (1\%, dilute stock $1: 100$ in $\left.\mathrm{dH}_{2} 0\right)$ for $24 \mathrm{~h}$.

\section{? TROUBLESHOOTING}


PROTOCOL

3| Day 2: add the PTU stock solution into the fish water to constitute a final concentration of $0.2 \mathrm{mM}$ (10x dilution) and incubate the embryos for an additional $24 \mathrm{~h}$.

I CAUTION PTU is used for preventing pigment formation in zebrafish embryos and is highly neurotoxic and carcinogenic. Thus, this chemical compound must be handled with a great caution in a chemical fume hood. PTU dishes and containers must be properly marked and handled only with gloves, and PTU waste should be kept in specific containers, which are disposed of separately.

4| Day 2 (continued): while incubating zebrafish embryos with PTU for $24 \mathrm{~h}$ (incubation is usually started in the morning) prepare tumor cells labeled with DiI dye. Use monolayers of tumor cells cultured in $75 \mathrm{~cm}^{3}$ culture flasks in DMEM supplemented with $10 \%$ (vol/vol) FBS, $1 \%$ (vol/vol) L-Glu and $1 \%$ (vol/vol) penicillin-streptomycin (FBS-DMEM, see REAGENT SETUP) at approximately $70-80 \%$ confluency. Add $1 \mu \mathrm{l}$ of $20 \mathrm{mg} \mathrm{ml}^{-1}$ DiI stock into $10 \mathrm{ml}$ of $1 \times$ DPBS (pre-equilibrated to room temperature) to achieve a final concentration of $2 \mu \mathrm{g} \mathrm{ml}^{-1}$. Discard the conditioned medium from the flasks and add $5 \mathrm{ml}$ of DPBS pre-equilibrated to room temperature to the flask shake gently and remove it. Add $10 \mathrm{ml}$ of the DiI labeling solution into each of the $75 \mathrm{~cm}^{3}$ culture flasks containing monolayers of tumor cells (Fig. 2); follow this by incubating cells at $37^{\circ} \mathrm{C}$ for $30 \mathrm{~min}$.

$\triangle$ CRITICAL STEP It is important to add the DiI into prewarmed 1× DPBS because DiI dye can solidify in cold DPBS. This is a crucial step to prevent blockage of capillary needles during the microinjection process of tumor cells into zebrafish embryos.

5| Collect all the medium containing the labeled tumor cells that become detached from the flasks as a result of DiI labeling and add it to a 50-ml Falcon tube; centrifuge at 1,000 g for 3 min and discard the supernatant.

? TROUBLESHOOTING

6| Wash labeled cells with $1 \times$ DPBS twice: add $\sim 5 \mathrm{ml}$ of DPBS into the tube containing cell pellet and resuspend the cells in DPBS by pipetting up and down using a 1-ml pipette; centrifuge at 1,000g for 3 min and discard the supernatant. Repeat this step one more time.

$\Delta$ CRITICAL STEP To remove all free dye particles from the cell suspension, labeled tumor cells should be washed thoroughly.

7| Add $2 \mathrm{ml}$ of $10 \%$ (vol/vol) FBS-DMEM (both fresh and pre-equilibrated to room temperature) and resuspend all the labeled cells with help of a pipette as described above; add the cell suspension into a new $75 \mathrm{~cm}^{3}$ flask containing $13 \mathrm{ml}$ of fresh $10 \%$ (vol/vol) FBS-DMEM without DiI, followed by incubation at $37^{\circ} \mathrm{C}$ overnight.

8| Day 3: Use two pairs of microsurgical forceps to dechorionate 48 h.p.f. embryos from Step 6. Hold the chorion with one forceps, and, with the help of the other forceps, grip the chorion and tear it apart (Fig. 2).

\section{? TROUBLESHOOTING}

9| Anesthetize the dechorionated zebrafish embryos by transferring them to fish water containing $0.004 \%$ (wt/vol) tricaine and wait for $\sim 2 \mathrm{~min}$.

10| Place up to ten embryos in each well and add $0.004 \%$ (wt/vol) tricaine water to each well of the $2 \%$ (wt/vol) modified agarose gel plate to precisely fill the wells. Excessive water can be removed with a Pasteur pipette.

$\Delta$ CRITICAL STEP If an excessive amount of water is used, zebrafish embryos can float outside the agarose

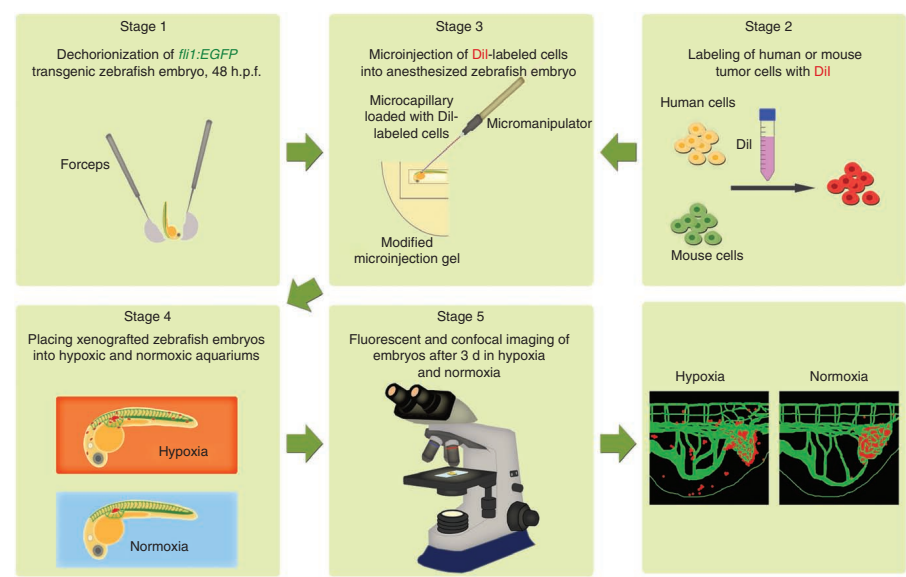

Figure 2 | Critical stages for tumor cell implantation into embryonic zebrafish and for analyzing tumor cell dissemination. fli1:EGFP zebrafish embryos at $48 \mathrm{~h}$ are dechorionized. Meanwhile, human or mouse tumor cells growing as monolayers are labeled with DiI dye and resuspended at an optimal concentration for microinjection. Approximately 100-150 tumor cells in $5 \mathrm{nl}$ are injected into the perivitelline space of each zebrafish embryo. Tumor-implanted zebrafish embryos are incubated in normoxic and hypoxic water for $3 \mathrm{~d}$. Dissemination of tumor cells can be detected using fluorescent microscopy at the single-cell level. Tumor cell (red) invasion and migration in association with tumor angiogenesis (green) or co-option of surrounding vasculatures in the host can be revealed using a two-channel fluorescent microscope. 
well, leading to empty wells. Additionally, excessive water can result in difficulties in positioning the zebrafish embryos at a fixed position for injection.

11| To prepare tumor cells from Step 7 for implantation, remove the medium and wash attached monolayers of tumor cells twice with $1 \times$ DPBS by adding $5 \mathrm{ml}$ of $1 \times$ DPBS into the flask, shaking the flask very gently and then removing the DPBS.

12 Add $2 \mathrm{ml}$ of $0.25 \%$ (wt/vol) trypsin pre-equilibrated to room temperature into the flask containing attached monolayer of tumor cells and incubate at room temperature for $30 \mathrm{~s}$. Remove the trypsin, tap the flask a few times very gently to help the cells to detach and stop trypsin activity by adding $2 \mathrm{ml} \mathrm{of} \mathrm{10 \%} \mathrm{(vol/vol)} \mathrm{FBS-DMEM.} \mathrm{Resuspend} \mathrm{the} \mathrm{tumor} \mathrm{cells}$ in the medium by pipetting up and down. Count the tumor cells under a phase contrast microscope. Centrifuge tumor cells at $1,000 \mathrm{~g}$ for $5 \mathrm{~min}$ and resuspend cells at a concentration of 20-30 cells per $\mathrm{nl}$ in $0.5-1 \%$ FBS-DMEM (e.g., FBS-DMEM as prepared in REAGENT SETUP, but with $0.5-1 \%$ FBS instead of $10 \%$ FBS).

$\Delta$ CRITICAL STEP It is crucial to stop trypsin activity by adding serum-containing cell culture medium. Otherwise, viability of cells can be markedly reduced during the counting of cells.

\section{? TROUBLESHOOTING}

13| Place the cell suspension on ice during the entire microinjection procedure.

14| Pull nonfilamentous borosilicate glass capillary needles using a micropipette puller. Load the microcapillary glass needle with $4 \mu \mathrm{l}$ of cell suspension and connect the needle to the micromanipulator. Point the needle tip to the injection site and gently insert the needle tip into the perivitelline space of the zebrafish embryo. Inject $5 \mathrm{nl}$ of the cell suspension containing approximately 100-150 cells into the perivitelline space of each embryo from Step 10 (Fig. 2).

\section{? TROUBLESHOOTING}

15| Transfer the injected zebrafish embryos to PTU water. The death rate of zebrafish embryos after tumor cell injection is $\sim 5 \%$.

\section{Hypoxia exposure period $\bigcirc$ TIMING $3 \mathrm{~d}$}

16| Fill the hypoxia chamber for zebrafish embryos with $1 \%$ (vol/vol) Danieu's water to the two-thirds level of the aquarium and calibrate the oxygen electrode using the same procedure described in adult zebrafish hypoxia model (Cao et al. ${ }^{7}$ ). Switch on the nitrogen gas and set the air saturation level to $7.5 \%$ as shown on the oxygen regulator (Fig. 1).

17| If drug treatment is being tested, add the chemical compound to aquarium water at the concentration that functionally intervenes with hypoxia-induced host responses (optional; see Experimental design).

18| When the hypoxia reaches a steady-state level, transfer tumor-implanted zebrafish embryos from Step 15 to the hypoxia chamber and close the lid. Incubate up to 100 tumor cell-implanted zebrafish embryos in the hypoxia chamber at $28{ }^{\circ} \mathrm{C}$.

$\triangle$ CRITICAL STEP A group of tumor-implanted zebrafish embryos should be placed in normoxia (regular water) to serve as a control.

$\Delta$ CRITICAL STEP Make sure that the lid is not completely sealed in order to avoid high pressure. If the lid is completely sealed, zebrafish embryos may die of anoxia.

19| Use a medium-sized magnet bar to mix the water and create a mild water current within the chamber to ensure that nitrogen gas is evenly distributed.

20| Maintain embryos in the hypoxia chamber for $3 \mathrm{~d}$.

$\Delta$ CRITICAL STEP The death rate is quite high at this hypoxia level; therefore, it is very important to closely monitor the chamber during the course of the entire experiment. Unlike in adult zebrafish experiments, there is no need to remove dead zebrafish embryos. To ensure that all zebrafish embryos are evenly exposed to the same hypoxic level, embryos should be swirling gently around in the chamber.

21| After $3 d$, transfer the tumor cell-implanted zebrafish embryos in water exposed to normoxia or hypoxia onto the surface of a $2 \%$ (wt/vol) agarose gel with help of a wide-tip Pasteur pipette for further analysis.

$\Delta$ CRITICAL STEP Analysis should be carried out immediately once the zebrafish embryos are placed on the agarose gel, as zebrafish embryos can only live for a few minutes in this condition. 


\section{Imaging TIMING 6-9 h}

22| At room temperature, cut a piece $(1 \mathrm{~cm} \times 1 \mathrm{~cm})$ of $2 \%(\mathrm{wt} / \mathrm{vol})$ agarose gel and place it on a glass slide. Use a Pasteur pipette to pick up a zebrafish embryo and carefully place it onto the gel cushion. Remove excessive water and add a small drop of $0.04 \%$ (wt/vol) tricaine onto the embryo.

$\Delta$ CRITICAL STEP If embryos are not completely anesthetized, increase the concentration of tricaine. Imaging should be performed immediately after anesthesia to reduce the risk of embryo death due to liquid evaporation.

23| Collect an embryo with a wide-tip Pasteur pipette and transfer it onto a glass slide; remove the excess water around using a paper tissue. If the paper tissue touches the embryo, the embryo will get dry and cannot be used for imaging. Put a small drop of Vectashield on the embryo, place a cover glass on it and seal the edges of the cover slide with nail polish. Keep the glass slide in a horizontal position in the dark for a short while until the nail polish dries and then transfer immediately to $-20^{\circ} \mathrm{C}$.

PAUSE POINT Mounted embryos at the horizontal position can be stored in dark at $-20^{\circ} \mathrm{C}$ for up to 2 weeks before microscopic examination.

24| Use fluorescent microscopy to analyze the number of disseminated tumor foci and cells. Perform imaging at low magnification ( $\times 4$ objective) using the ultraviolet-activated filter at the wavelength of $488 \mathrm{~nm}$. The fluorescent light filter allows excitement of EGFP in zebrafish embryos. Use filters for green fluorescence to visualize zebrafish embryo vasculature and filters for red fluorescence to image implanted tumor cells labeled with DiI. To illuminate the DiI-labeled tumor cells in zebrafish embryos, use green light at the wavelength of $555 \mathrm{~nm}$. It is essential to capture green fluorescent-positive and red fluorescent-positive signals at the same position on the embryos in order to associate DiI-labeled tumor cells with blood vessels by superimposing green and red signals. Typically, for each zebrafish embryo, two different sets of images from the head region and the tail region are collected as the separate green fluorescent-positive signals, the red fluorescentpositive signals, and the merged signals. All disseminated tumor cell signals in each embryo should be counted. At least 13-20 embryos in each experimental group should be used to obtain statistically significant values.

25| Use three-dimensional (3D) confocal microscopy to study angiogenesis and metastasis in more detail. Confocal low magnification ( $\times 4$ objective) can be used to image the whole body to get an overview of the tumor cell dissemination pattern, and higher magnifications ( $\times 20$ and $\times 40$ objectives) are suitable for studying intratumoral and peritumoral angiogenesis and also for precise localization of disseminated cells and foci inside the zebrafish embryo body. Use a 488-nm laser to scan the zebrafish embryo vasculature and a 543-nm laser to scan DiI-labeled implanted tumor cells. To achieve a high-quality 3D image, each embryo should be scanned in eight to ten layers and each layer should be scanned six times to remove the background and get a sharp picture (Fig. 3).

26| To quantify the number of disseminated cells and foci, count all the cells and foci that have disseminated from the tumor mass toward the fish embryo head and tail using fluorescence software, ImageJ or any other kind of useful software program. For further data analysis, use Excel or similar software to calculate the average number of disseminated foci and cells. In addition, to measure the distance of metastasis, choose the focus or cell that lies furthest away from the tumor mass and measure the distance between that focus and the primary tumor. The programs and software mentioned above can also be used for this purpose. Adobe Photoshop can be used to measure and analyze the ratio of tumor vessel density relative to size and tumor volume; see the accompanying paper $^{7}$ for more details.
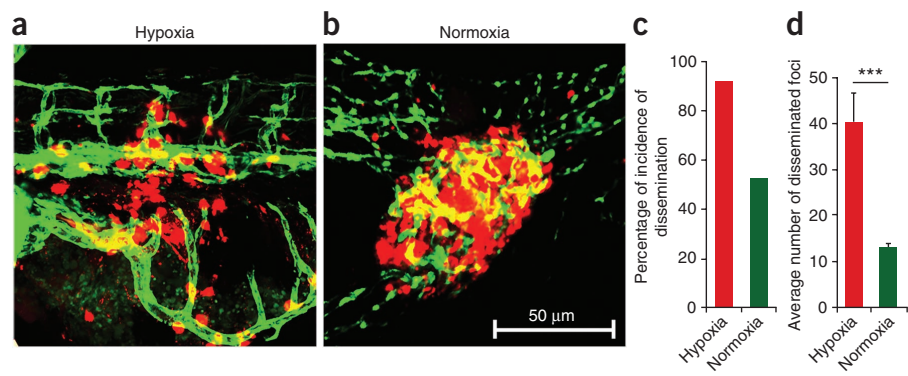

Figure 3 | Dissemination and quantification of disseminated mouse T241 fibrosarcoma cells. Approximately 100 DiI-labeled mouse T241 fibrosarcoma cells were injected into the perivitelline space of each 48 h.p.f. fli1:EGFP zebrafish embryo. The tumor cell-implanted zebrafish embryos are exposed to normoxic or hypoxic water at $28^{\circ} \mathrm{C}$ for $72 \mathrm{~h}$. Green color represents zebrafish embryo blood vessels, red color represents DiI-labeled tumor cells and yellow color shows overlapping positive signals. (a,b) Tumor cell dissemination and metastases under hypoxic conditions (a) and angiogenesis under normoxia (b) were detected using confocal microscopy. Scale bar applies to both panels $\mathbf{a}$ and $\mathbf{b}$. (c) Percentage of zebrafish carrying disseminated tumor cells (total number of embryos studied was 13 for hypoxia and 15 for normoxia) under normoxia and hypoxia were quantified. All DiI-positive cells located outside the primary tumor mass were manually counted as disseminated cells or foci using the software mentioned in Step 26. (d) The average number of disseminated foci from primary tumor implanted in zebrafish embryos (13 for hypoxia and 15 for normoxia) were quantified. (Adapted from ref. 4 with permission.) Error bars indicate the standard deviation; asterisks stand for $P<0.001$ and show the significant difference between the two groups. 


\section{? TROUBLESHOOTING}

Troubleshooting advice can be found in Table 1.

TABLE 1 | Troubleshooting table.

\begin{tabular}{|c|c|c|c|}
\hline Step & Problem & Possible reason & Solution \\
\hline 2 & Too few eggs & $\begin{array}{l}\text { Nonproductive } \\
\text { breeding pairs }\end{array}$ & $\begin{array}{l}\text { Choose a few healthy, well-fed breeding pairs of zebrafish to } \\
\text { ensure enough eggs }\end{array}$ \\
\hline 5 & $\begin{array}{l}\text { Tumor cells remain attached after } 0.5-\mathrm{h} \\
\text { incubation with labeling medium }\end{array}$ & $\begin{array}{l}\text { Highly adherent } \\
\text { cell line }\end{array}$ & $\begin{array}{l}\text { Release cells by resuspending them in the labeling medium using } \\
\text { a 1-ml pipette tip. Avoid using trypsin at this stage }\end{array}$ \\
\hline 8 & $\begin{array}{l}\text { Embryos are destroyed during } \\
\text { dechorionization }\end{array}$ & $\begin{array}{l}\text { Inexperienced } \\
\text { and less skillful } \\
\text { personnel }\end{array}$ & $\begin{array}{l}\text { Practice by starting with a large number of embryos and choose } \\
\text { perfect ones for injection }\end{array}$ \\
\hline 12 & Formation of cell clusters & Dead cell clumps & $\begin{array}{l}\text { Add } 0.5 \% \text { FBS to the medium to reduce trypsin-induced } \\
\text { cell death }\end{array}$ \\
\hline 14 & Blockage of injection capillaries & $\begin{array}{l}\text { Formation of } \\
\text { clumps and too } \\
\text { much cell debris }\end{array}$ & $\begin{array}{l}\text { Load the microcapillary needle with the desired volume of cell } \\
\text { suspension, pipette up and down a couple of times, remove the } \\
\text { suspension and load the needle again or increase cell density } \\
\text { and reduce the injection volume }\end{array}$ \\
\hline
\end{tabular}

\section{TIMING}

Step 1, Breeding (day 0): $15 \mathrm{~h}$

Step 2, Incubation of embryos in Danieu's solution (day 1): $\sim 24 \mathrm{~h}$

Steps 3-7, Incubation of embryos in $0.2 \mathrm{mM}$ PTU solution (day 2): $\sim 24 \mathrm{~h}$

Steps 8-15, Dechorionization and tumor cell injection in zebrafish embryo (day 3): 2-3 h per 100 embryos

Steps 16-21, Hypoxia exposure period: $3 \mathrm{~d}$

Steps 22-26, Imaging: 6-9 h

\section{ANTICIPATED RESULTS}

\section{Hypoxia-induced dissemination of tumor cells in embryonic zebrafish}

Using this protocol, dissemination of DiI-labeled (T241, fibrosarcoma) tumor cells can be detected at single-cell level using a fluorescent microscope (stage 5 in Figs. 2 and 3). Moreover, hypoxia-induced tumor angiogenesis (green color) in relation to dissemination of tumor cells (red color) can be monitored using a two-channel confocal image analysis. Notably, a majority of disseminated tumor cells are associated with the vasculature, demonstrating the essential role of angiogenesis in tumor invasion and metastasis. As the primary tumor remains at a relatively small size during the entire experimental procedure, hypoxic exposure is unlikely to generate an uneven gradient that causes tumor cells to be superficially invasive owing to co-option to surrounding healthy vasculature. Figure $\mathbf{3}$ shows an example of the expected results of metastasis under hypoxia using DiI-labeled mouse fibrosarcoma cells with quantification of metastases.

ACKNOWLEDGMENTS Y.C.'s laboratory was supported by research grants from the Swedish Research Council, the Swedish Cancer Foundation, the Karolinska Institute Foundation, the Karolinska Institute distinguished professor award, the European Union Integrated Project of Metoxia (Project no. 222741) and the European Research Council (ERC) advanced grant ANGIOFAT (Project no 250021). Z.C. is supported by the Swedish Heart and Lung Foundation.

AUTHOR CONTRIBUTIONS Y.C. designed the study. L.D.J., P.R. and Z.C. performed the experiments. L.D.J., P.R., Z.C. and Y.C. analyzed the data. K.H., T.L., J.F.S. and E.W. participated in designing and discussing this study. L.D.J., P.R., Z.C. and Y.C. wrote the paper.

COMPETING FINANCIAL INTERESTS The authors declare no competing financial interests.
Published online at http://www.natureprotocols.com/.

Reprints and permissions information is available online at http://npg.nature. com/reprintsandpermissions/.

1. Erler, J.T. et al. Hypoxia-induced lysyl oxidase is a critical mediator of bone marrow cell recruitment to form the premetastatic niche. Cancer Cell 15, 35-44 (2009).

2. Rouhi, P. et al. Pathological angiogenesis facilitates tumor cell dissemination and metastasis. Cell Cycle 9, 913-917 (2010).

3. Cao, Y. Opinion: emerging mechanisms of tumour lymphangiogenesis and lymphatic metastasis. Nat. Rev. Cancer 5, 735-743 (2005)

4. Lee, S.L. et al. Hypoxia-induced pathological angiogenesis mediates tumor cell dissemination, invasion, and metastasis in a zebrafish tumor model. Proc. Natl. Acad. Sci. USA 106, 19485-19490 (2009). 


\section{PROTOCOL}

5. Nissen, L.J. et al. Angiogenic factors FGF2 and PDGF-BB synergistically promote murine tumor neovascularization and metastasis. J. Clin. Invest. 117, 2766-2777 (2007).

6. Cao, R. et al. PDGF-BB induces intratumoral lymphangiogenesis and promotes lymphatic metastasis. Cancer Cell 6, 333-345 (2004).

7. Cao, Z. et al. Hypoxia-induced retinopathy model in adult zebrafish. Nat. Protoc. 12, 1903-1910 (2010)
8. van Rooijen, E. et al. Zebrafish mutants in the von Hippel-Lindau tumor suppressor display a hypoxic response and recapitulate key aspects of Chuvash polycythemia. Blood 113, 6449-6460 (2009)

9. Lawson, N.D. \& Weinstein, B.M. In vivo imaging of embryonic vascular development using transgenic zebrafish. Dev. Biol. 248, 307-318 (2002). 10. Jin, S.W., Beis, D., Mitchell, T., Chen, J.N. \& Stainier, D.Y. Cellular and molecular analyses of vascular tube and lumen formation in zebrafish. Development 132, 5199-5209 (2005). 
PAPER 



\title{
Anti-VEGF- and anti-VEGF receptor-induced vascular alteration in mouse healthy tissues
}

\author{
Yunlong Yang a,b,1, Yin Zhang ${ }^{\mathrm{a}, 1}$, Ziquan Cao ${ }^{c, 1}$, Hong $\mathrm{Ji}^{\mathrm{a}}$, Xiaojuan Yang ${ }^{\mathrm{a}}$, Hideki Iwamoto ${ }^{\mathrm{a}}$, Eric Wahlbergc, \\ Toste Lännec, Baocun Sun ${ }^{\mathrm{d}}$, and Yihai Cao ${ }^{\mathrm{a}, \mathrm{c}, 2}$
}

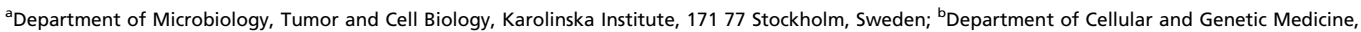
Shanghai Medical College, Fudan University, Shanghai 200032, China; 'Department of Medicine and Health Sciences, Linköping University, 58185 Linköping, Sweden; and ${ }^{\mathrm{d} D e p a r t m e n t}$ of Pathology, Tianjin Medical University, Tianjin 300070, China

Edited by Tadamitsu Kishimoto, Immunology Frontier Research Center, Osaka University, Suita, Japan, and approved May 22, 2013 (received for review January 21, 2013)

Systemic therapy with anti-VEGF drugs such as bevacizumab is widely used for treatment of human patients with various solid tumors. However, systemic impacts of such drugs in host healthy vasculatures remain poorly understood. Here, we show that, in mice, systemic delivery of an anti-VEGF or an anti-VEGF receptor (VEGFR)-2 neutralizing antibody caused global vascular regression. Among all examined tissues, vasculatures in endocrine glands, intestinal villi, and uterus are the most affected in response to VEGF or VEGFR-2 blockades. Thyroid vascular fenestrations were virtually completely blocked by VEGF blockade, leading to marked accumulation of intraendothelial caveolae vesicles. VEGF blockade markedly increased thyroid endothelial cell apoptosis, and withdrawal of anti-VEGF resulted in full recovery of vascular density and architecture after $14 \mathrm{~d}$. Prolonged anti-VEGF treatment resulted in a significant decrease of the circulating level of the predominant thyroid hormone free thyroxine, but not the minimal isoform of triiodothyronine, suggesting that chronic anti-VEGF treatment impairs thyroid functions. Conversely, VEGFR-1-specific blockade produced virtually no obvious phenotypes. These findings provide structural and functional bases of anti-VEGF-specific drug-induced side effects in relation to vascular changes in healthy tissues. Understanding antiVEGF drug-induced vascular alterations in healthy tissues is crucial to minimize and even to avoid adverse effects produced by currently used anti-VEGF-specific drugs.

angiogenesis | vascular homeostasis | vessel regression |

antiangiogenic therapy $\mid$ off-tumor targets

$T^{T}$ he antiangiogenic concept for treatment of solid tumors was proposed by Judah Folkman $41 \mathrm{y}$ ago (1). In this hypothetic paper, Folkman wrote that "one approach to the initiation of 'antiangiogenesis' would be the production of an antibody against tumor angiogenic factor." Today, the most commonly used anti-VEGF drug, bevacizumab (avastin) (2), is a humanized neutralizing antibody against tumor-derived VEGF, validating Folkman's early prediction.

Tumors produce various angiogenic factors and cytokines to induce angiogenesis that is essential for tumor growth. Among these tumor-derived factors, VEGF, also called VEGF-A, is a key angiogenic factor that induces angiogenesis, vascular permeability, and tortuosity $(3,4)$. VEGF displays these broad vascular functions via binding and activation of its specific receptors, VEGFR-1 and VEGFR-2, mainly expressed in vascular endothelial cells, although other cell types may also express these receptors (5-8). Abundant experimental data demonstrate that VEGFR-2 is the primary functional receptor that transduces both angiogenic and vascular permeability signals whereas VEGFR-1 may function as a decoy receptor $(9,10)$. It should be emphasized that, under physiological and pathological conditions, VEGFR-2 is expressed not only in angiogenic vessels, but also in quiescent vasculatures in various tissues (11). The broad distribution of VEGFRs in quiescent vasculatures in various healthy tissues suggests that the VEGF-VEGFR signaling system is essential for maintenance of vascular homeostasis. In some tissues, such as endocrine organs including adrenal glands, thyroid, and pancreatic islets, the endothelium in these vasculatures remains fenestrated and VEGF is crucial for maintenance of vascular fenestrations (12-14). Kamba et al. reported that systemic delivery of tyrosine kinase inhibitors (TKIs) containing the anti-VEGFR component caused vascular regression in various tissues (15). However, TKIs have broad targets, and it was unclear whether blocking VEGFR signaling was solely responsible for the vascular phenotype.

In the present study, we chose to use VEGF- and VEGFRspecific blockades that do not target other signaling systems. Additionally, we have also distinguished the significance of VEGFR1- and VEGFR-2-mediated signaling pathways in maintenance of vascular homeostasis in various tissues and organs. Given the fact that bevacizumab is the most commonly used antiangiogenic drug in oncology (16-21), our findings using these specific blockades may be directly translated into clinical relevance concerning the global impact of these drugs in cancer patients and the structural basis of adverse effects. Thus, the information provided in our study could be potentially useful for development of new therapeutic strategies to minimize or avoid adverse effects of antiVEGF-based therapeutics.

\section{Results}

Impact of Anti-VEGF Blockades on Vasculature in Endocrine Organs. To study the impact of VEGF-specific blockades on vasculatures in various healthy tissues, we used three specific anti-VEGF agents known to block VEGF-induced biological activities: a rabbit anti-mouse neutralizing monoclonal antibody (BD0801) (22); a rat anti-mouse VEGFR-1 neutralizing monoclonal antibody (MF-1) (23-25); and a rat anti-mouse VEGFR-2 neutralizing monoclonal antibody (DC101) (23-25). These antibodies were systemically delivered for $2 \mathrm{wk}$ to healthy mice using doses known to block tumor angiogenesis (22-25). Because bevacizumab (a humanized anti-human VEGF neutralizing monoclonal antibody) is widely being used for treatment of various human cancers $(2,17-21)$ and ramucirumab (a humanized antihuman VEGFR-2 neutralizing monoclonal antibody) together with docetaxel-based chemotherapy is planned for a phase III trial for treatment of non-small cell lung carcinoma (26), systemic treatment with the same agents designed in our study would be clinically relevant.

Auther contributions: Y.C. designed research: $Y . Y$, Y.Z Z, Z.C., H.J., X.Y., and H.I. performed research; E.W., T.L., and B.S. contributed new reagents/analytic tools; Y.Y., Y.Z., and Z.C. analyzed data; and Y.C. wrote the paper.

The authors declare no conflict of interest.

This article is a PNAS Direct Submission.

${ }^{1} Y . Y .$, Y.Z., and Z.C. contributed equally to this work.

${ }^{2}$ To whom correspondence should be addressed. E-mail: yihai.cao@ki.se.

This article contains supporting information online at www.pnas.org/lookup/suppl/doi:10. 1073/pnas.1301331110/-/DCSupplemental. 


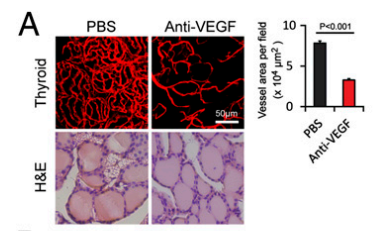

B

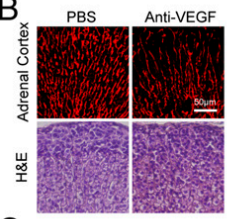

$\mathrm{C}_{\text {PBS }}$
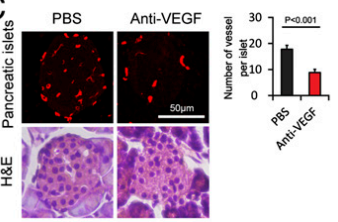
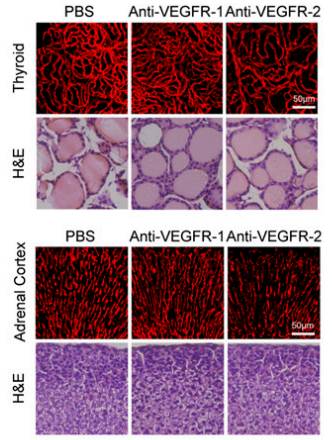

PBS

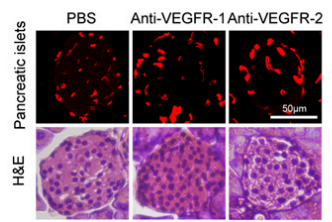

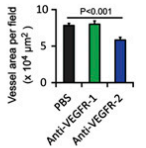
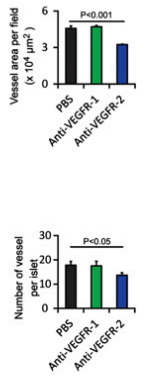

Fig. 1. Impact of anti-VEGF blockades on vasculature in endocrine organs. ( $A$ ) $\mathrm{CD} 31^{+}$thyroid microvessels (red). H\&E staining was used to reveal tissue structures. Vessel areas were quantified (20x magnification, $n=8$ fields per group). ( $B$ ) Endomucin ${ }^{+}$adrenal cortex microvessels were detected in paraffin-embedded tissues (red). H\&E staining was used to reveal tissue structures. Vessel areas were quantified (20x magnification, $n=8$ fields per group). (C) Endomucin $^{+}$pancreatic islet microvessels were detected in paraffin-embedded tissues (red). H\&E staining was used to reveal tissue structures. Vessel numbers were quantified (20× magnification, $n=8$ fields per group). Data are presented as means \pm SEM.

To define the receptor type that is involved in maintenance of VEGF-dependent vascular homeostasis, VEGFR-1- and VEGFR2-specific blockades were systemically delivered to mice. Consistent with the known receptor functions, the VEGFR-2-specific blockade produced a similar vascular regression activity in these endocrine organs (Fig. $1 A-C$ ). However, delivery of the VEGFR-1 blockade resulted in virtually no repressive effects on the vasculature in these tissues. These data show that VEGFR-2 is the critical receptor that mediates the VEGF-dependent maintenance function in these endocrine organs.

Vascular Response in Gastrointestinal Tracts and the Female Reproductive System. Examination of vasculatures in the gastric wall, small intestinal wall, and colon wall showed significant
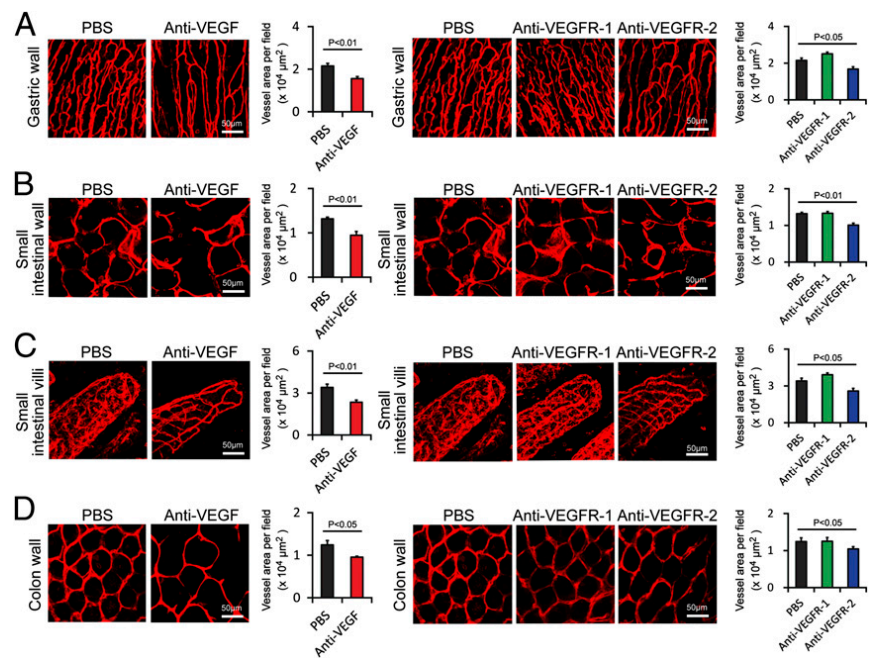

Fig. 2. Impact of anti-VEGF blockades on vasculature in gastrointestinal tracts. $(A) \mathrm{CD} 31^{+}$gastric wall microvessels (red). Vessel areas were quantified $(20 \times$ magnification, $n=8$ fields per group). (B) $\mathrm{CD} 1^{+}$small intestine wall (red). Vessel areas were quantified (20X magnification, $n=8$ fields per group). (C) CD $31^{+}$small intestine villi microvessels (red). Vessel areas were quantified (20x magnification, $n=$ fields 8 per group). (D) $\mathrm{CD}_{3} 1^{+}$colon wall microvessels in anti-VEGF or buffer-treated healthy mice were detected using $\mathrm{CD} 31^{+}$ endothelial cell signals (red). Vessel areas were quantified ( $20 \times$ magnification, $n=8$ fields per group). Data are presented as means \pm SEM. 

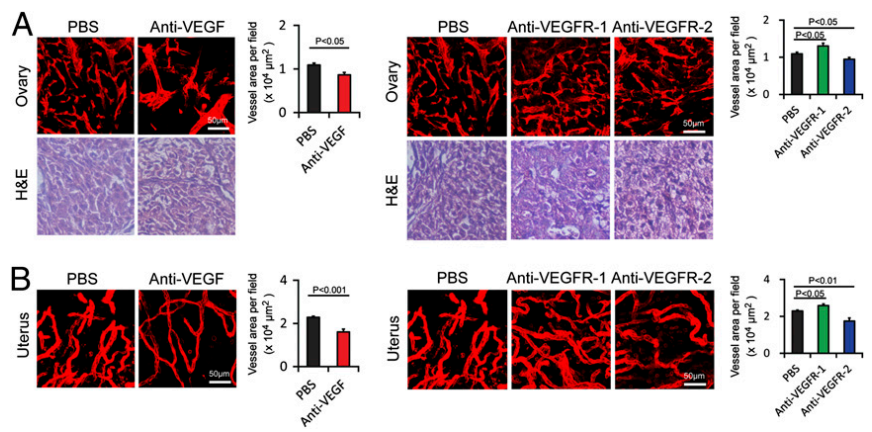

Fig. 3. Impact of anti-VEGF blockades on vasculature in female reproductive system. (A) $\mathrm{CD} 31^{+}$ovary microvessels (red). H\&E staining was used to reveal tissue structures. Vessel areas were quantified (20x magnification, $n=8$ fields per group). (B) $\mathrm{CD}_{3} 1^{+}$uterus microvessels (red). Vessel areas were quantified (20X magnification, $n=8$ per fields group). Data are presented as means \pm SEM. reduction of microvessel density in response to the VEGF blockade (Fig. $2 A, B$, and $D$ ). A similar vascular regressive effect was also found in the VEGFR-2-treated groups. Intriguingly, microvessels in small intestinal villi showed marked decrease of vessel density in response to VEGF or VEGFR-2 blockade (Fig. 2C). However, systemic treatment with the VEGFR-1-specific blockade did not produce any obvious vascular changes (Fig. $2 A-D$ ). In the ovary, systemic administration of VEGF and VEGFR-2 blockades produced significant effects on vascular regression whereas VEGFR-1 blockade did not show any vascular regressive effect. Surprisingly, treatment with the VEGFR-1 blockade increased vessel density in the ovarian tissue (Fig. 3A). There were no obvious structural changes upon anti-VEGF blockades as detected by hematoxylin/eosin histological staining. Similar to the ovary tissue, VEGF- and VEGFR-2-specific blockades significantly decreased vascular density in the uterus whereas VEGFR-1 increased vascular density in this tissue (Fig. 3B). These data demonstrate that the VEGF-VEGFR-2 signaling system is essentially required for maintenance of a subset of vasculatures in these tissues and organs.

Vascular Changes in Kidney, Liver, Pancreas, and Other Tissues. Among all analyzed tissues, renal cortex and glomeruli in the kidney showed significant reduction of vascular density in response to the VEGF-specific blockade (Fig. $4 A$ and $B$ ). The hepatic sinusoidal vasculature also responded to the VEGFspecific blockade, leading to a significant reduction of microvessel density (Fig. 4C). In the pancreatic acini area, the antiVEGF treatment resulted in significant decrease of vessel numbers (Fig. 4D). Consistent with these findings, systemic delivery of the VEGFR-2 blockade produced a similar vascular regressive phenotype in these tissues whereas anti-VEGFR-1 blockade did not affect the vessel density (Fig. $4 A-D$ ). We have also examined vasculatures in a number of other tissues including different regions of the brain, retina, thymus, myocardium, skeletal muscles, and bone marrow. In general, no significant changes were observed in these tissues and organs in response to the three VEGF blockades, with the exception of
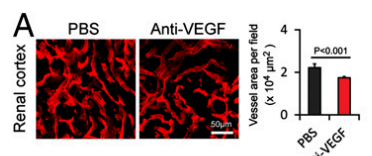

B PBS Anti-VEGF
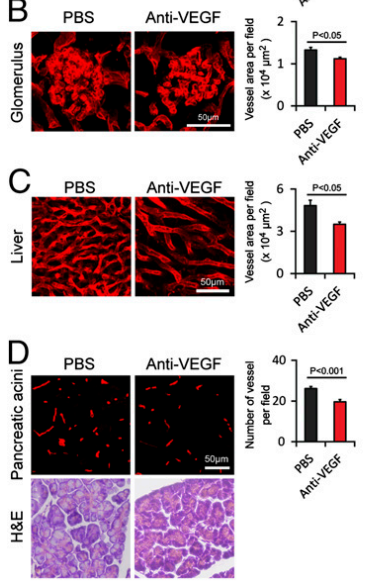
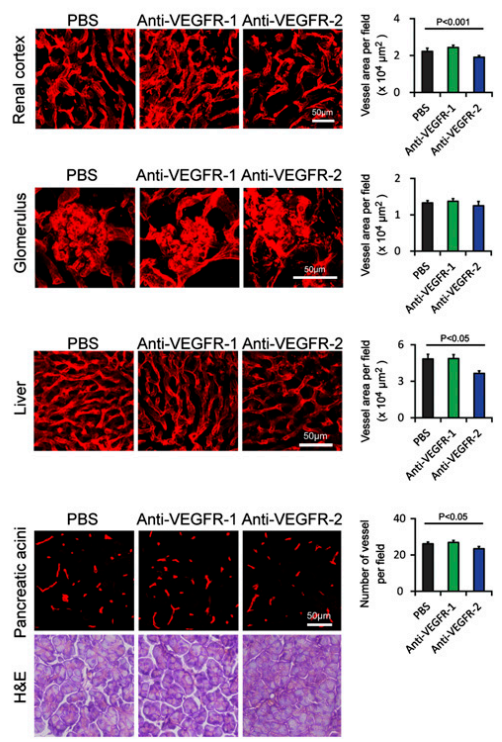

Fig. 4. Impact of anti-VEGF blockades on vasculature in kidney, liver, and pancreas. $(A) C D 31^{+}$renal cortex microvessels (red). Vessel areas were quantified (20x magnification, $n=8$ fields per group). (B) $\mathrm{CD} 31^{+}$glomerulus microvessels (red). Vessel areas were quantified (20× magnification, $n=8$ fields per group). (C) CD $31^{+}$ liver microvessels (red). Vessel areas were quantified (20x magnification, $n=8$ fields per group). (D) Endomucin $^{+}$pancreatic acini microvessels were detected in paraffin-embedded tissues (red). H\&E staining was used to reveal tissue structures. Vessel numbers were quantified ( $20 \times$ magnification, $n=8$ fields per group). Data are presented as means \pm SEM. 
significant reduction of vascular density in thymus in response to VEGF and VEGFR-2 blockades (Figs. S1-S6).

Reversible Vascular Density and Architecture Recovery. Consistent with anti-VEGF-induced vascular regression, a substantial number of endothelial cells in anti-VEGF-treated thyroid vessels underwent cellular apoptosis with expression of the activated caspase- 3 in endomucin ${ }^{+}$endothelial structures (Fig. $5 A$ ). In fact, a more than sevenfold increase of apoptotic endothelial cell percentage was detected in the anti-VEGFtreated group relative to the untreated control group (Fig. 5A). Despite the existence of a substantial number of apoptotic endothelial cells in anti-VEGF-treated samples, thyroid follicular and other nonendothelial cells rarely became apoptotic during the 2-wk treatment period. In concordance with vascular reduction, anti-VEGF-treated thyroid tissue exhibited significant tissue hypoxia (Fig. $5 G$ ). Vascular blockade also induced thyroid vessel regression in a dose-dependent manner without altering vessel diameters (Fig. 5D). To study the anti-VEGFinduced vascular rarefaction, we performed an "on-off" experiment in which anti-VEGF drugs were delivered to healthy mice for $2 \mathrm{wk}$, followed by analysis of the thyroid vasculature at different time points (Fig. $5 B$ ). Notably, anti-VEGF-induced thyroid vascular regression was nearly completely recovered after 2-wk cessation of VEGF blockade, and vascular density and architecture returned to the same levels seen in the untreated animals (Fig. 5C). However, we did not observe a "rebound" effect. These data demonstrate that anti-VEGF-induced vascular rarefaction is completely reversible after discontinuation of antiVEGF treatment.

Anti-VEGF-Induced Functional Impacts in Thyroid Gland. AntiVEGF-induced thyroid vessel regression promoted us to study the function impact of VEGF blockade. First, we measured thyroid tissue blood perfusion in anti-VEGF-treated and nontreated groups. In agreement with vascular density reduction, anti-VEGF-treated thyroid exhibited marked reduction of blood perfusion as measured using fluorescein-labeled 2,000-kDa dextran (Fig. 5E). However, blood perfusion in each individual vessel in anti-VEGF-treated and nontreated groups was not altered (Fig. 5E). Blood vessels in endocrine organs are known to contain fenestrated endothelium, and vascular fenestrations are crucial for maintenance of endocrine organ functions $(28,29)$. Furthermore, VEGF is the crucial factor for induction and maintenance of vascular fenestrations in endocrine organs. We next investigated the impact of anti-VEGF treatment on alteration of vascular fenestrations in thyroid gland. As expected, thyroid gland contained highly fenestrated microvasculatures with approximately more than 2 fenestrae per $\mu \mathrm{m}$ (Fig. $5 H$ ). Remarkably, VEGF blockade virtually completely suppressed
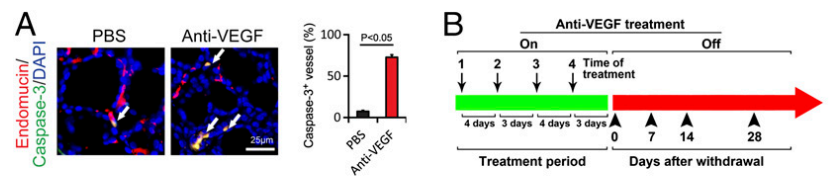

C
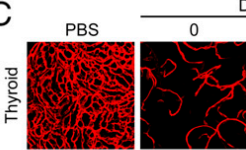

Days after
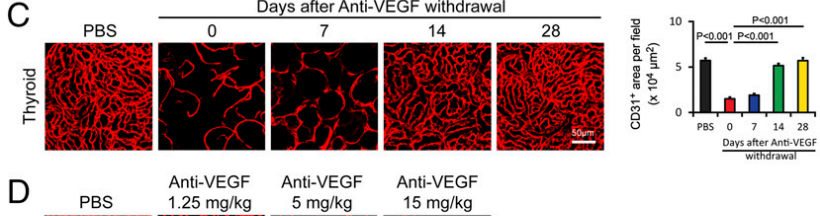

Anti-VEGF
$1.25 \mathrm{mg} / \mathrm{kg}$

Anti-VEGF

Anti-VEGF
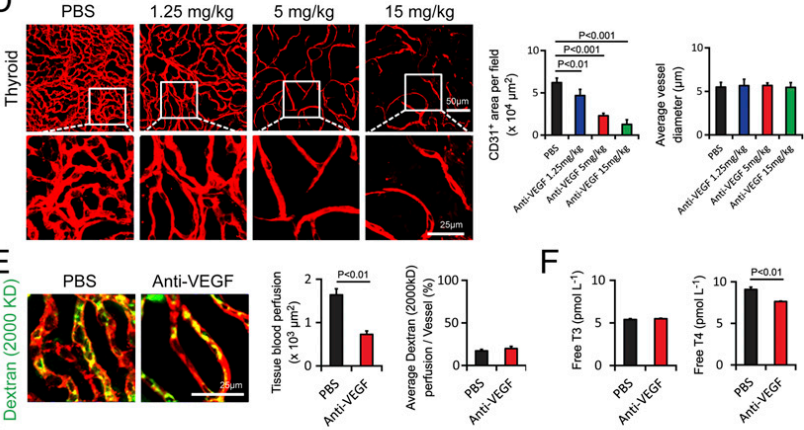

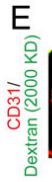

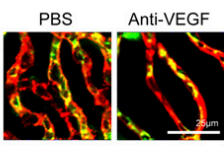

G Thyroid PBS VEGF

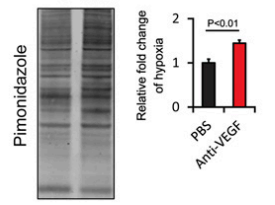

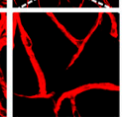

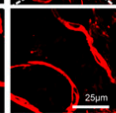

F

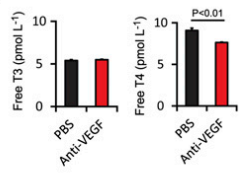

H

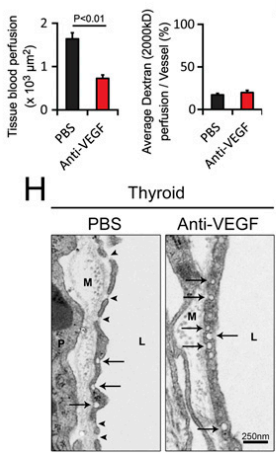

Fig. 5. Endothelial cell apoptosis, cessation-induced vascular recovery, time course, tissue hypoxia, vescular fenestration, and thyroid functional alterations in response to anti-VEGF treatment. (A) At $2 \mathrm{wk}$ after treatment with VEGF blockade, paraffin-embedded thyroid tissue sections were triple immunostained with an anti-caspase-3 antibody (green), an anti-endomucin antibody (red), and DAPI (blue). Arrows point to endothelial apoptotic cells. Caspase$3^{+}$apoptotic endothelial cells were quantified $(n=8$ per group). ( $B$ ) Schematic diagram showing the treatment regimen and cessation of anti-VEGF therapy. On, on drug; Off, off drug. (C) Thyroid vascular alterations in response to VEGF blockade and withdrawal of treatment at various time points. $\mathrm{CD} 31^{+}$microvessels in thyroid tissues of antiVEGF-treated and nontreated groups were quantified ( $n=$ 8 fields per group). (D) Thyroid vascular alterations in response to various doses of VEGF blockade and quantification of vascular $\mathrm{CD} 31^{+}$density and the average of vascular diameter ( $n=8$ fields per group). ( $E$ ) Vascular perfusions were double immunostained with $\mathrm{CD} 31$ (red) and a lysinated fluorescein-labeled dextran (2,000 kDa; green). Total tissue and individual vessel perfusion were quantified $(n=$ 8 fields per group). ( $F$ ) Serum levels of thyroid hormone free T3 and free T4 as measured using an ELISA method ( $n$ $=3$ samples per group). (G) Quantification of tissue hypoxia in response to anti-VEGF treatment $(n=3$ samples per group). (H) Thyroid vascular fenestrations in anti-VEGFtreated and nontreated tissue samples and numbers of fenestrae and caveolae were quantified ( $n=8$ samples per group). M, matrix; P, perivascular cell; $L$, lumen. Arrows point to caveolae, and arrowheads indicate fenestrae. Data are presented as means \pm SEM. 
the formation of endothelial fenestrations in thyroid vessels (Fig. $5 H$ ), suggesting that VEGF in thyroid acts as a homeostatic factor for maintenance of vascular fenestrations. Interestingly, concomitant with reduction of vascular fenestrations, the number of endothelial caveolae was significantly increased in antiVEGF-treated thyroid tissues relative to the vehicle-treated group (Fig. $5 H$ ). To further study the functional impact of antiVEGF treatment in modulation of thyroid gland functions, mice were treated with VEGF blockade for a prolonged period of 4 wk. Importantly, the circulating level of the predominant thyroid hormone free thyroxine (T4), but not the minimal isoform of free triiodothyronine (T3), was significantly decreased in the VEGF blockade-treated mice compared with that of the vehicle-treated group (Fig. $5 F$ ). These findings provide compelling evidence of the endocrine functional impairment of thyroid gland after prolonged treatment with VEGF blockade.

\section{Discussion}

Since the US Food and Drug Administration (FDA) approval of the first antiangiogenic drug, bevacizumab, for treatment of metastatic colorectal cancer in 2004 (2), this anti-VEGF drug has been widely used for treatment of various human cancers, including ovarian, breast, lung, glioblastoma, and renal cell carcinoma (RCC) $(17-21,30,31)$. In general, addition of bevacizumab to the standard chemotherapy significantly improves beneficial effects in cancer patients although the bevacizumabrelated overall survival benefits remain modest (32). Bevacizumab in combination with chemotherapy has become the first-line standard care for treatment of several cancer types, particularly for renal cell carcinoma, non-small cell lung carcinoma, colorectal cancer, and gastrointestinal stromal tumors (19-21, 33). Additionally, ramucirumab and aflibercept, as VEGFR-2-specific and VEGF trap, respectively, are under consideration or already approved for FDA approval for oncology use (34-36). In fact, several FDA-approved TKIs that block VEGFRs, such as sunitinib, sorafenib, and pazopanib, are also commonly used antiangiogenic drugs in the clinic for treatment of human patients with RCC and other cancer types (37-39). Clinical experiences with bevacizumab and other antiVEGF agents show that these drugs produce a range of adverse effects commonly seen in cancer patients, including hypertension, renal vascular injury often manifested by proteinuria and thrombotic microangiopathy, gastrointestinal perforation, and congestive heart failure (40-42). The anti-VEGF agent-induced broad adverse effects demonstrate that these drugs have a broad impact on vasculatures in multiple healthy tissues and organs. Although this important issue has been known for a long time, there are limited data and knowledge on how these drugs affect healthy vasculatures in various tissues. In the present study, we chose to study the systemic impact of VEGF-specific inhibitors on mice to recapitulate the clinical situation.

Among all analyzed tissues and organs, the most affected vasculatures in response to mouse VEGF and VEGFR-2 blockades have been observed in endocrine organs, particularly in thyroid. It is known that anti-VEGF agents could trigger hypothyroidism (43), suggesting that they potentially target vasculatures in the thyroid gland in human patients. We have also observed marked vascular changes in pancreatic islets where insulin-producing $\beta$-cells are located. The clinical significance of the anti-VEGFinduced vascular changes in pancreatic islets remains unknown. Similarly, clinical relevance of the anti-VEGF-induced vascular changes in adrenal cortex needs to be established. Based on our findings, we can reasonably conclude that VEGF is essentially required for maintenance of vascular homeostasis in certain endocrine organs. In the established vasculature in endocrine organs, VEGF, together with several other vascular factors, may display two main functions: maintenance of endothelial cell survivals and vascular fenestrations. We further provide evidence showing that anti-VEGF-induced thyroid endothelial apoptosis as a potential mechanism of vascular regression, indicating that VEGF is an essential survival factor of vasculatures in endocrine glands. In the same line of this view, treatment with VEGF blockade almost completely blocked vascular fenestrations in thyroid vessels. These findings reconcile with the known vascular survival and permeability functions of $\operatorname{VEGF}(9,14)$. Another interesting aspect of our study is that anti-VEGFR-2, but not anti-VEGFR-1, produced similar effects as VEGF blockade, demonstrating that VEGFR-2 is the primary functional receptor that transduces VEGF-mediated vascular homeostasis. Inversely, in some tissues, VEGFR-1 blockade produced a trend or significant effect to increase rather than to decrease vascular density in some tissues, suggesting that VEGFR-1 negatively regulates VEGF functions. In fact, such a negative function of VEGFR-1 in regulation of angiogenesis and homeostasis has been thoroughly discussed elsewhere (9).

Despite induction of endothelial apoptosis, cessation of VEGF blockade treatment resulted in a rapid and reversible recovery of vascular density and architecture of the thyroid vasculature, suggesting that regressed vessels can be regenerated. Although the detailed mechanism underlying vascular regeneration warrants further investigation, there was a similar regenerative mechanism of anti-VEGF-treated tumor vessels, in which angiogenic vessels grow along the basement sheet mainly made by collagen IV (44). Another interesting point is that withdrawal of VEGF blockade takes a relatively short time (approximate $2 \mathrm{wk}$ ) to fully recover the regressed vasculature despite the long half-life of the antibody. One possible explanation is that anti-VEGF treatment itself markedly increases VEGF expression levels in various tissues as seen in non-tumor-bearing mice (45). In supporting this view, anti-VEGF-treated tissues such as thyroid show an increased level of hypoxia, a known factor that markedly upregulates VEGF expression levels $(46,47)$. High levels of VEGF rapidly neutralize the unbound free antibody molecules, resulting in defective neutralization of the antibody to VEGF. Within a relatively short period of anti-VEGF treatment, thyroid gland and perhaps other endocrine organs, exhibits extremely high capacity of compensatory functional recovery in response to vascular regression, leading to no obvious functional changes. However, prolonged treatment and persistent exposure to VEGF blockade resulted in low-level production of endocrine hormones such as thyroxine. Functional impairment of thyroid functions by chronic exposure to VEGF blockade could result from two vascular defects: vascular density reduction and elimination of vascular fenestrations.

Anti-VEGF-induced vascular changes in glomeruli may, in part, explain this type of side effect in human cancer patients (48, 49). Our data also show that VEGF-dependent vascular plasticity occurs in a tissue- and organ-specific manner. Of note, endocrine abnormalities seem to be uncommon during clinical practice of antiangiogenic drugs (50). Vasculatures in several other organs and tissues such as myocardium, brain, and skeletal muscles remain unaffected despite vascular exposure to the same dose of anti-VEGF drugs.

Taken together, our preclinical findings with systemic delivery of anti-VEGF-specific drugs in mice are in general concordance with clinically manifested adverse effects caused by these drugs in human cancer patients. Thus, our data provide a structural basis of broad targets in healthy tissues and also reveal the physiological functions of VEGF in maintenance of vascular homeostasis in various tissues and organs. Understanding the broad impacts of these anti-VEGF-specific drugs may help us to minimize or even to avoid side effects in future clinical practice.

\section{Methods}

All animal studies were approved by the Northern Stockholm Experimenta Animal Ethical Committee (Stockholm, Sweden). Statistical analyses were performed using the standard two-tailed Student $t$ test. Details are provided in SI Methods. 
ACKNOWLEDGMENTS. We thank Imclone for providing VEGFR-1- and VEGFR2-specific neutralizing antibodies and Simcere Pharmaceuticals for providing VEGF blockade. We thank Dr. Kjell Hultenby for technical support in electron microscopy work. Y.C.'s laboratory is supported by research grants from the Swedish Research Council, the Swedish Cancer Foundation, the Karolinska Institute Foundation, the Karolinska Institute Distinguished Professor Award,

1. Folkman J (1971) Tumor angiogenesis: Therapeutic implications. N Engl J Med 285 (21): $1182-1186$.

2. Hurwitz H, et al. (2004) Bevacizumab plus irinotecan, fluorouracil, and leucovorin for metastatic colorectal cancer. N Engl J Med 350(23):2335-2342.

3. Senger DR, et al. (1983) Tumor cells secrete a vascular permeability factor that promotes accumulation of ascites fluid. Science 219(4587):983-985.

4. Leung DW, Cachianes G, Kuang WJ, Goeddel DV, Ferrara N (1989) Vascular endothelial growth factor is a secreted angiogenic mitogen. Science 246(4935):1306-1309. 5. Coultas L, Chawengsaksophak K, Rossant J (2005) Endothelial cells and VEGF in vascular development. Nature 438(7070):937-945.

6. Carmeliet P, Jain RK (2000) Angiogenesis in cancer and other diseases. Nature 407 (6801):249-257.

7. Yancopoulos GD, et al. (2000) Vascular-specific growth factors and blood vessel formation. Nature 407(6801):242-248.

8. Ferrara N, Gerber HP, LeCouter J (2003) The biology of VEGF and its receptors. Nat Med 9(6):669-676.

9. Cao Y (2009) Positive and negative modulation of angiogenesis by VEGFR1 ligands. Sci Signal 2(59):re1.

10. Sato $Y$, et al. (2000) Properties of two VEGF receptors, Flt-1 and KDR, in signal transduction. Ann N Y Acad Sci 902:201-205, discussion 205-207.

11. Witmer AN, Dai J, Weich HA, Vrensen GF, Schlingemann RO (2002) Expression of vascular endothelial growth factor receptors 1,2 , and 3 in quiescent endothelia. $J$ Histochem Cytochem 50(6):767-777.

12. LeCouter J, et al. (2001) Identification of an angiogenic mitogen selective for endocrine gland endothelium. Nature 412(6850):877-884.

13. Vittet D, Ciais D, Keramidas M, De Fraipont F, Feige JJ (2000) Paracrine control of the adult adrenal cortex vasculature by vascular endothelial growth factor. Endocr Res 26 (4):843-852.

14. Eriksson A, et al. (2003) Small GTP-binding protein Rac is an essential mediator of vascular endothelial growth factor-induced endothelial fenestrations and vascular permeability. Circulation 107(11):1532-1538.

15. Kamba T, et al. (2006) VEGF-dependent plasticity of fenestrated capillaries in the normal adult microvasculature. Am J Physiol Heart Circ Physiol 290(2):H560-H576.

16. Ignoffo RJ (2004) Overview of bevacizumab: A new cancer therapeutic strategy targeting vascular endothelial growth factor. Am J Health Syst Pharm 61(21, Suppl 5) S21-S26.

17. Miklos GL (2012) Bevacizumab in neoadjuvant treatment for breast cancer. N Engl Med 366(17):1638, author reply 1639-1640.

18. Mountzios G, Pentheroudakis G (2012) Bevacizumab in ovarian cancer. N Engl J Med 366(13):1257, author reply 1258 .

19. Sculier JP, Meert AP, Paesmans M (2007) Bevacizumab for non-small-cell lung cancer. N Engl J Med 356(13):1373-1374, author reply 1374-1375.

20. Sharieff W (2004) Bevacizumab in colorectal cancer. N Engl J Med 351(16):1690-1691; author reply $1690-1691$

21. Sonpavde G (2003) Bevacizumab in renal-cell cancer. N Engl J Med 349(17):1674.

22. $\mathrm{Yu} Y$, et al. (2010) A humanized anti-VEGF rabbit monoclonal antibody inhibits angiogenesis and blocks tumor growth in xenograft models. PLOS ONE 5(2):e9072.

23. Xue $Y$, et al. (2008) Anti-VEGF agents confer survival advantages to tumor-bearing mice by improving cancer-associated systemic syndrome. Proc Natl Acad Sci USA 105 (47): $18513-18518$.

24. Zhang $D$, et al. (2011) Antiangiogenic agents significantly improve survival in tumorbearing mice by increasing tolerance to chemotherapy-induced toxicity. Proc Nat Acad Sci USA 108(10):4117-4122.

25. Cao R, et al. (2010) VEGFR1-mediated pericyte ablation links VEGF and PIGF to cancerassociated retinopathy. Proc Natl Acad Sci USA 107(2):856-861. the Torsten Söderbergs Foundation, Söderbergs Stiftelse, the Tianjin Natural Science Foundation (Center for Molecular Medicine-Tianjin Grant 09ZCZDSF04400) for international collaboration between Tianjin Medical University and Karolinska Institutet, ImClone Systems Inc/EliLilly, the European Union Integrated Project of Metoxia (Project 222741), and European Research Council Advanced Grant ANGIOFAT (Project 250021)

26. Garon EB, et al. (2012) A randomized, double-blind phase III study of docetaxel and ramucirumab versus docetaxel and placebo in the treatment of stage IV non-smallcell lung cancer after disease progression after 1 previous platinum-based therapy (REVEL): Treatment rationale and study design. Clin Lung Cancer 13(6):505-509.

27. Fan L, Iseki S (1998) Immunohistochemical localization of vascular endothelial growth factor in the endocrine glands of the rat. Arch Histol Cytol 61(1):17-28.

28. Palade GE, Simionescu M, Simionescu N (1979) Structural aspects of the permeability of the microvascular endothelium. Acta Physiol Scand Suppl 463:11-32.

29. Renkin EM (1979) Relation of capillary morphology to transport of fluid and large molecules: a review. Acta Physiol Scand Suppl 463:81-91.

30. Wong ET, Brem S (2011) Taming glioblastoma by targeting angiogenesis: 3 years later. J Clin Oncol 29(2):124-126.

31. Friedman HS, et al. (2009) Bevacizumab alone and in combination with irinotecan in recurrent glioblastoma. J Clin Oncol 27(28):4733-4740.

32. Kerbel RS (2008) Tumor angiogenesis. N Engl J Med 358(19):2039-2049.

33. Lièvre A, Landi B, Mitry E, Taïeb J (2008) [Antiangiogenic agents and gastrointestinal cancers]. Gastroenterol Clin Biol 32(5 Pt 1):504-520.

34. Spratlin JL, Mulder KE, Mackey JR (2010) Ramucirumab (IMC-1121B): A novel attack on angiogenesis. Future Oncol 6(7):1085-1094.

35. Spratlin $\mathrm{J}$, et al. (2010) Phase I pharmacologic and biologic study of ramucirumab (IMC-1121B), a fully human immunoglobulin G1 monoclonal antibody targeting the vascular endothelial growth factor receptor-2. J Clin Oncol 28(5):780-787.

36. Ramlau R, et al. (2012) Aflibercept and Docetaxel versus Docetaxel alone after platinum failure in patients with advanced or metastatic non-small-cell lung cancer: A randomized, controlled phase III trial. J Clin Oncol 30(29):3640-3647.

37. Motzer RJ, et al. (2007) Sunitinib versus interferon alfa in metastatic renal-cell carcinoma. N Engl J Med 356(2):115-124.

38. Escudier B, et al.; TARGET Study Group (2007) Sorafenib in advanced clear-cell renalcell carcinoma. N Engl J Med 356(2):125-134.

39. Bramwell VH (2012) Pazopanib and the treatment palette for soft-tissue sarcoma. Lancet 379(9829):1854-1856.

40. des Guetz G, Uzzan B, Chouahnia K, Morère JF (2011) Cardiovascular toxicity of antiangiogenic drugs. Target Oncol 6(4):197-202.

41. Randall LM, Monk BJ (2010) Bevacizumab toxicities and their management in ovarian cancer. Gynecol Oncol 117(3):497-504.

42. Tol J, et al. (2008) Gastrointestinal ulceration as a possible side effect of bevacizumab which may herald perforation. Invest New Drugs 26(4):393-397.

43. Rini BI (2011) Review: Thyroid function abnormalities in patients receiving VEGFtargeted therapy. Clin Adv Hematol Oncol 9(4):337-338.

44. Mancuso MR, et al. (2006) Rapid vascular regrowth in tumors after reversal of VEGF inhibition. J Clin Invest 116(10):2610-2621.

45. Ebos JM, Lee CR, Christensen JG, Mutsaers AJ, Kerbel RS (2007) Multiple circulating proangiogenic factors induced by sunitinib malate are tumor-independent and correlate with antitumor efficacy. Proc Natl Acad Sci USA 104(43):17069-17074.

46. Shweiki D, Itin A, Soffer D, Keshet E (1992) Vascular endothelial growth factor induced by hypoxia may mediate hypoxia-initiated angiogenesis. Nature 359(6398): 843-845.

47. Makino $Y$, et al. (2001) Inhibitory PAS domain protein is a negative regulator of hypoxia-inducible gene expression. Nature 414(6863):550-554.

48. Launay-Vacher V, Deray G (2009) Hypertension and proteinuria: A class-effect of antiangiogenic therapies. Anticancer Drugs 20(1):81-82.

49. Zhu X, Wu S, Dahut WL, Parikh CR (2007) Risks of proteinuria and hypertension with bevacizumab, an antibody against vascular endothelial growth factor: Systematic review and meta-analysis. Am J Kidney Dis 49(2):186-193.

50. Perren TJ, et al.; ICON7 Investigators (2011) A phase 3 trial of bevacizumab in ovarian cancer. N Engl J Med 365(26):2484-2496. 


\section{Supporting Information}

\section{Yang et al. 10.1073/pnas.1301331110 \\ SI Methods}

Mice. Female 8- to 12 -wk-old C57BL/6 mice were bred, acclimated, and caged in a group of six or fewer mice per cage at the animal facility of the Department of Microbiology, Tumor and Cell Biology, Karolinska Institute. Mice were killed by a lethal dose of $\mathrm{CO}_{2}$, followed by cervical dislocation. All animal studies were approved by the Karolinska Institutet local ethical committee and the Northern Stockholm Experimental Animal Ethical Committee.

Treatment with VEGF and VEGFR Blockades. A rabbit anti-mouse VEGF-specific neutralizing antibody (BD0801) was kindly provided by the Simcere Pharmaceutical Company (Nanjing, Jiangsu, China) (1). Rat specific antibodies to mouse VEGF receptor (VEGFR)-1 (MF1) and to mouse VEGFR-2 (DC101) (2-4) were kindly provided by ImClone Systems. These antibodies were intraperitoneally (i.p.) injected twice per week into each of the mice at the dose of $5 \mathrm{mg} / \mathrm{kg}$ of the anti-VEGF antibody and $200 \mu \mathrm{g}$ per mouse of anti-VEGFR-1 or anti-VEGFR-2 antibodies ( $n=6$ mice per group). Tissue samples were collected at the end of the experimental duration. In the discontinuation experiment, VEGF blockade was injected i.p. two times/week into each of the mice at the dose of $5 \mathrm{mg} / \mathrm{kg}$ ( $n=6$ mice per group). Tissue samples were collected at various time points after cessation of anti-VEGF treatment, including day $0,7,14$, and 28. For dose-dependent experiments, VEGF blockade was injected i.p. two times per week into each of the mice at the doses of $1.25,5$, and $15 \mathrm{mg} / \mathrm{kg}$ ( $n=6$ mice per group).

Tissue, Blood and Organ Collection. Mice were killed after 2 wk of antibody administration. Necropsy was performed, and various tissues and organs were removed and immediately fixed with $4 \%$ (wt/vol) Paraformaldehyde (PFA) overnight, followed by washing with PBS. A fraction of the tissues and organs was embedded with paraffin until further use. Blood samples were collected from anti-VEGF and control animals using an intracardial method $(2,3)$, and serum samples were kept at $-20{ }^{\circ} \mathrm{C}$ until further use.

Histological Studies and Whole-Mount Staining. Paraffin-embedded tissues were sectioned at a thickness of $5 \mu \mathrm{m}$ and stained with $\mathrm{H} \& \mathrm{E}$ as described (5). Whole-mount staining was performed according to our published methods (6-11). Briefly, small pieces of tissues were cut into thin slices and fixed in $4 \%$ (wt/vol) PFA overnight, followed by treatment with proteinase $\mathrm{K}(20 \mu \mathrm{g} / \mathrm{mL})$. Tissues were incubated with primary antibodies overnight at $4{ }^{\circ} \mathrm{C}$, followed by staining with secondary antibodies for $2 \mathrm{~h}$ at room temperature. Necessary washes with PBS were performed in between or after staining. Slides were mounted and examined under a confocal microscope (Zeiss Confocal LSM510 Microscope). By scanning 10 thin sections at $4-$ to $5-\mu \mathrm{m}$ distances of each sample, three-dimensional images of each tissue sample were projected. Quantitative analyses from at least eight different tissue sections were performed using Adobe Photoshop CS software program.

Immunofluorescence Staining. Paraffin-embedded slides were prepared at $5 \mu \mathrm{m}$ and baked at $60{ }^{\circ} \mathrm{C}$ overnight. Antigen retrieval was achieved with an unmasking solution (Vector Labs; H3300). Bone samples were preheated for $2.5 \mathrm{~min}$ in a microwave oven at the maximal power and then heated for $5.5 \mathrm{~min}$ at $20 \%$ power. For soft samples, prolonged heating was used. Samples were chilled to room temperature, followed by washing twice with PBS for $5 \mathrm{~min}$. Samples were treated with blocking buffer [4\% (vol/ vol) goat serum in PBS] at room temperature for $30 \mathrm{~min}$. A rat anti-mouse endomucin (eBioscience; cat. no.14-5851-85, clone eBioV.7C7) antibody (1:200 dilution in blocking buffer) was used for incubation at $4{ }^{\circ} \mathrm{C}$ overnight. A secondary antibody (goat anti-rat Alexa 555; Invitrogen; cat. no. A21434; 1:400 dilution) in the blocking buffer was incubated at room temperature for $45 \mathrm{~min}$. For apoptosis assay, a rabbit anti-mouse cleaved caspase-3 antibody (Cell Signaling; cat. no. 9661, 1:200 dilution in a blocking buffer) and a secondary antibody (donkey antirabbit Alexa 488; Invitrogen; cat. no. A21206; 1:400 dilution) were used. Slides were mounted with Vectashield (Vector Laboratories; cat. no. H-1000) and kept in $-20{ }^{\circ} \mathrm{C}$.

Vascular Perfusion Assay. Anti-VEGF-treated and nontreated mice were anesthetized and were injected into the tail vein with a lysinated fluorescein-labeled dextran $(2,000 \mathrm{kDa} ; 100 \mu \mathrm{L}$ per mouse; Invitrogen) (12). After $10 \mathrm{~min}$, animals were killed, and tissues and organs were dissected and immediately fixed with $4 \%$ (wt/vol) PFA at $4{ }^{\circ} \mathrm{C}$. Thyroids were carefully dissected, wholemount stained, and examined by confocal microscopy.

Western Blot. After 2-wk anti-VEGF treatment, mice were anesthetized and i.v. injected with $1.5 \mathrm{mg}$ pimonidazole (Hypoxyprobe). Mice were killed 15 min later, and thyroid was resected and immediately lysed. Total protein samples were collected. Fifteen micrograms of total protein was loaded into each channel and separated using a $10 \%(\mathrm{wt} / \mathrm{vol})$ SDS/PAGE gel and then transferred onto a nitrocellulose membrane. Membranes were probed overnight at $4{ }^{\circ} \mathrm{C}$ with a mouse anti-pimonidazole monoclonal antibody (clone 4.3.11.3, Hypoxyprobe) and diluted in PBS with $5 \%$ (wt/vol) BSA containing $0.1 \%$ Tween 20 , as recommended by the manufacturer. The membrane was incubated for $1 \mathrm{~h}$ with a donkey anti-mouse IgG antibody (IRDye 680RD; LI-COR; 1:15000). Protein bands were visualized and quantified using ODYSSEY CLX (LI-COR) at $700 \mathrm{~nm}$ wavelength.

ELISA. Serum levels of mouse free triiodothyronine (T3) and free thyroxine (T4) were measured and quantified by using an ELISA method according to the manufacturer's instructions (cat. nos. MBS705057 and MBS162146; Mybiosource).

Electron Microscopy. After 2-wk anti-VEGF antibody treatment, animals were killed and immediately fixed with $2.5 \%$ (vol $/ \mathrm{vol}$ ) glutaraldehyde plus $1 \%$ paraformaldehyde in $0.1 \mathrm{M}$ phosphate buffer ( $\mathrm{pH} 7.4$ ) by vascular perfusion. Thyroids were dissected and fixed with a fresh fixative. Electron microscopy examination and image captures were obtained as previously described (13, 14). Ultrathin tissue sections in $40-50 \mathrm{~nm}$ were prepared and examined in a Tecnai 12 Spirit Bio TWIN transmission electron microscope (Fei Company) at $100 \mathrm{kV}$. Digital images were taken by using a Veleta camera (Olympus Soft Imaging Solutions).

Statistical Analysis. For quantitative analysis, randomized micrographs from at least eight different fields were used. Six animals were recruited to each group, and each experiment was repeated three times. The Adobe Photoshop CS4 software program was used with a color range tool and a count tool to detect positive areas or numbers. Statistical analyses were performed using the standard two-tailed Student $t$ test, and values of $P<0.05, P<$ 0.01 , and $P<0.001$ were deemed statistically significant. 


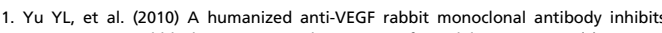
angiogenesis and blocks tumor growth in xenograft models. PLOS ONE 5(2):e9072.

2. Xue $Y$, et al. (2008) Anti-VEGF agents confer survival advantages to tumor-bearing mice by improving cancer-associated systemic syndrome. Proc Natl Acad Sci USA 105 (47): $18513-18518$

3. Zhang $D$, et al. (2011) Antiangiogenic agents significantly improve survival in tumorbearing mice by increasing tolerance to chemotherapy-induced toxicity. Proc Nat/ Acad Sci USA 108(10):4117-4122.

4. Cao R, et al. (2010) VEGFR1-mediated pericyte ablation links VEGF and PIGF to cancerassociated retinopathy. Proc Natl Acad Sci USA 107(2):856-861.

5. Xue $Y$, et al. (2012) PDGF-BB modulates hematopoiesis and tumor angiogenesis by inducing erythropoietin production in stromal cells. Nat Med 18(1):100-110.

6. Cao R, et al. (2012) Collaborative interplay between FGF-2 and VEGF-C promotes lymphangiogenesis and metastasis. Proc Natl Acad Sci USA 109(39):15894-15899.

7. Zhang $F$, et al. (2012) Proliferative and survival effects of PUMA promote angiogenesis. Cell Rep 2(5):1272-1285.

8. Jensen LD, et al. (2012) Opposing effects of circadian clock genes bmal1 and period2 in regulation of VEGF-dependent angiogenesis in developing zebrafish. Cell Rep 2(2): $231-241$
9. Lim S, et al. (2012) Cold-induced activation of brown adipose tissue and adipose angiogenesis in mice. Nat Protoc 7(3):606-615.

10. Cao R, et al. (2011) Mouse corneal lymphangiogenesis model. Nat Protoc 6(6): 817-826.

11. Xue $Y$, Lim S, Bråkenhielm E, Cao $Y$ (2010) Adipose angiogenesis: Quantitative methods to study microvessel growth, regression and remodeling in vivo. Nat Protoc 5 (5):912-920.

12. Hedlund EM, et al. (2013) Tumor cell-derived placental growth factor sensitize antiangiogenic and antitumor effects of anti-VEGF drugs. Proc Natl Acad Sci USA 110 (2):654-659.

13. Al-Hashmi S, et al. (2012) Busulphan-cyclophosphamide cause endothelial injury, remodeling of resistance arteries and enhanced expression of endothelial nitric oxide synthase. PLOS ONE 7(1):e30897.

14. Eriksson A, et al. (2003) Small GTP-binding protein Rac is an essential mediator of vascular endothelial growth factor-induced endothelial fenestrations and vascular permeability. Circulation 107(11):1532-1538. 

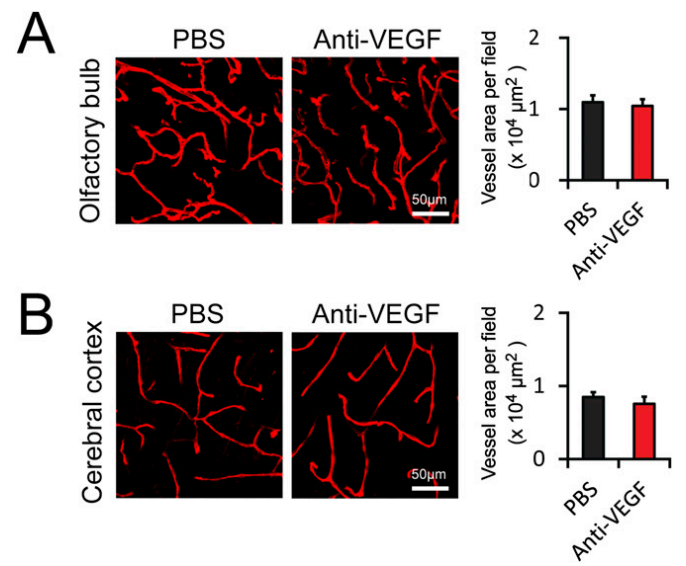

0
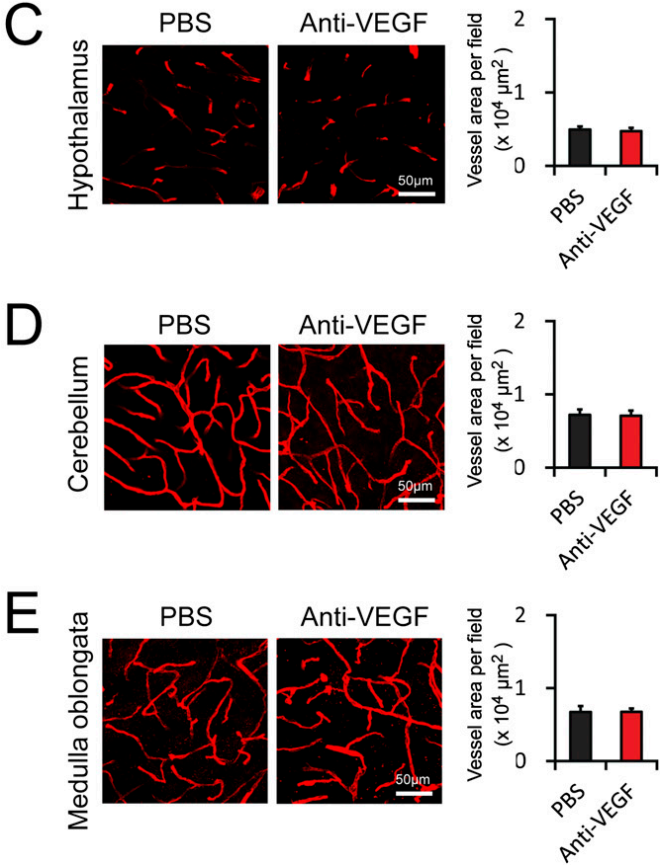

Fig. S1. Impact of anti-VEGF antibody treatment on vasculature in brain. $(A) C D 31^{+}$olfactory bulb microvessels (red). Vessel areas were quantified (20 $\times$

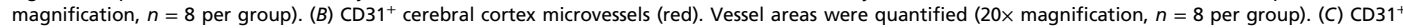
hypothalamus microvessels (red). Vessel areas were quantified ( $20 \times$ magnification, $n=8$ per group). (D) $C D 31^{+}$cerebellum microvessels (red). Vessel areas were

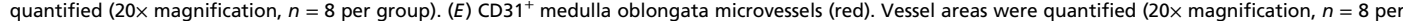
group). Data are presented as means \pm SEM. 

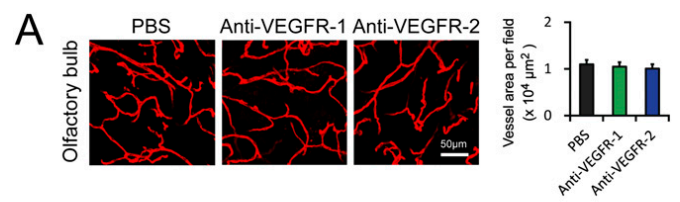

B
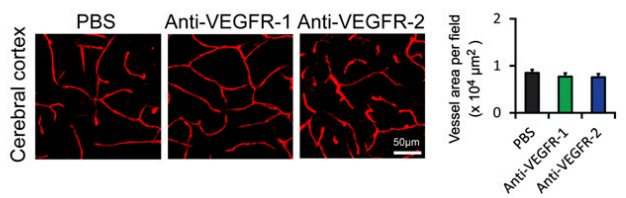

C
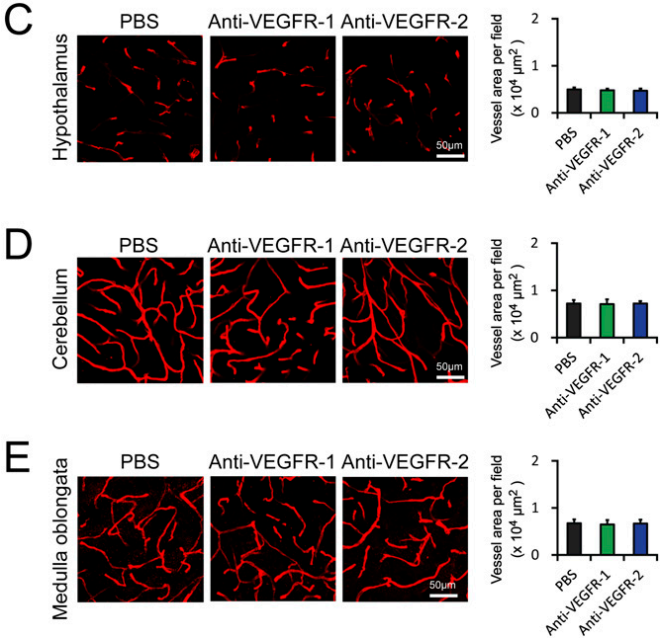

Fig. S2. Impact of anti-VEGFR-1 and anti-VEGFR-2 antibody treatment on vasculature in brain. (A) CD $31^{+}$olfactory bulb microvessels (red). Vessel areas were quantified ( $20 \times$ magnification, $n=8$ per group). $(B) C D 31^{+}$cerebral cortex microvessels (red). Vessel areas were quantified (20× magnification, $n=8$ per group). (C) CD31 $1^{+}$hypothalamus microvessels (red). Vessel areas were quantified ( $20 \times$ magnification, $n=8$ per group). (D) CD31 ${ }^{+}$cerebellum microvessels (red). Vessel areas were quantified (20x magnification, $n=8$ per group). (E) CD $31^{+}$medulla oblongata microvessels (red). Vessel areas were quantified (20x magnification, $n=8$ per group). Data are presented as means \pm SEM. 

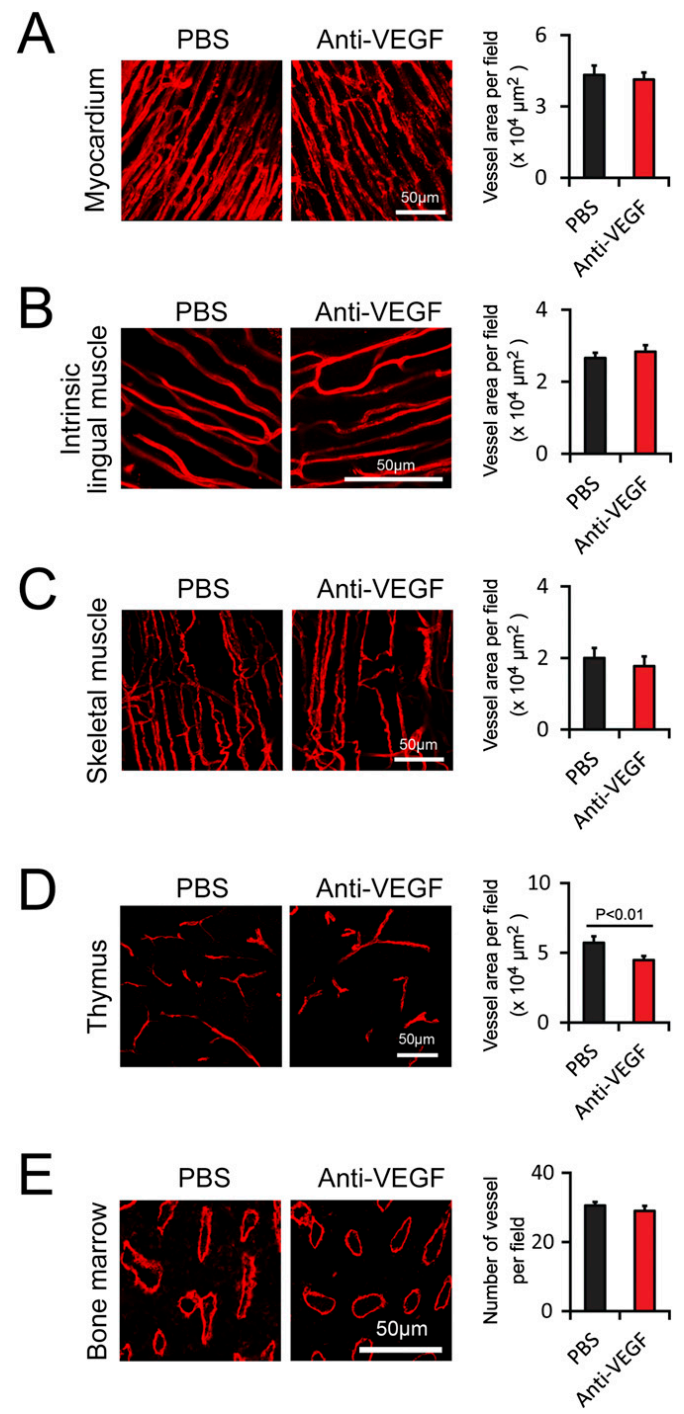

Fig. S3. Impact of anti-VEGF antibody treatment on vasculature in muscle, thymus, and bone marrow. $(A) \mathrm{CD} 31^{+}$myocardium microvessels (red). Vessel areas

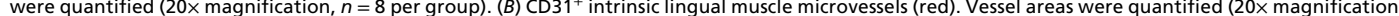
$n=8$ per group). (C) $C D 31^{+}$skeletal muscle microvessels (red). Vessel areas were quantified (20 $\times$magnification, $n=8$ per group). (D) CD31 $1^{+}$thymus microvessels (red). Vessel areas were quantified ( $20 \times$ magnification, $n=8$ per group). ( $E$ ) Endomucin ${ }^{+}$bone marrow microvessels were detected in paraffinembedded (red). Vessel areas were quantified (20× magnification, $n=8$ per group). Data are presented as means \pm SEM 
A

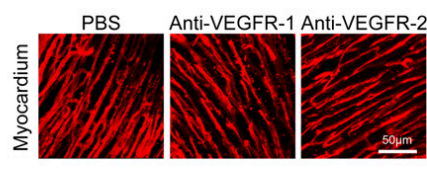

B

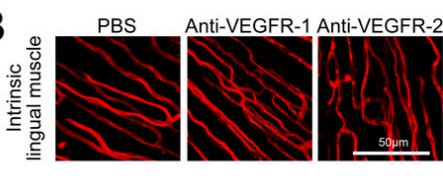

C

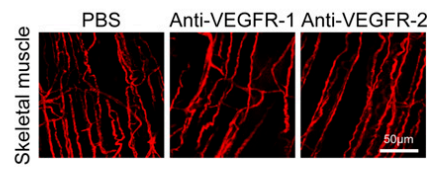

D

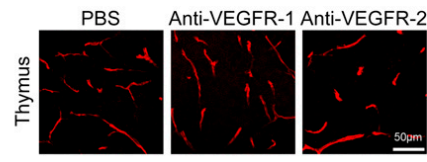

E

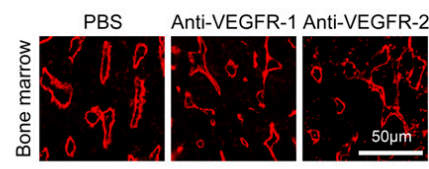

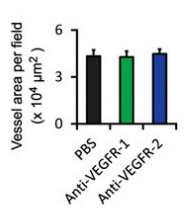
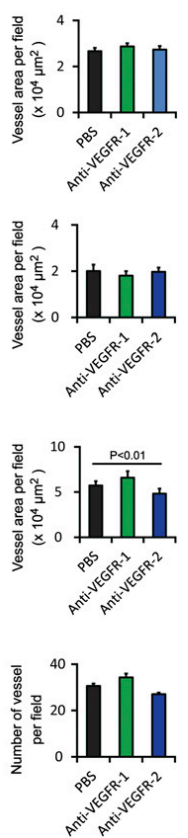

Fig. S4. Impact of anti-VEGFR-1 and anti-VEGFR-2 antibody treatment on vasculature in muscle, thymus, and bone marrow. $(A)$ CD $31^{+}$myocardium microvessels (red). Vessel areas were quantified ( $20 \times$ magnification, $n=8$ per group). $(B) C D 31^{+}$intrinsic lingual muscle microvessels (red). Vessel areas were

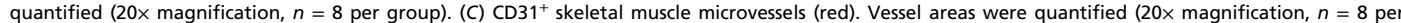
group). ( $D$ ) $\mathrm{CD}_{3} 1^{+}$thymus microvessels (red). Vessel areas were quantified ( $20 \times$ magnification, $n=8$ per group). ( $E$ ) Endomucin ${ }^{+}$bone marrow microvessels were detected in paraffin-embedded (red). Vessel areas were quantified ( $20 \times$ magnification, $n=8$ per group). Data are presented as means \pm SEM. 

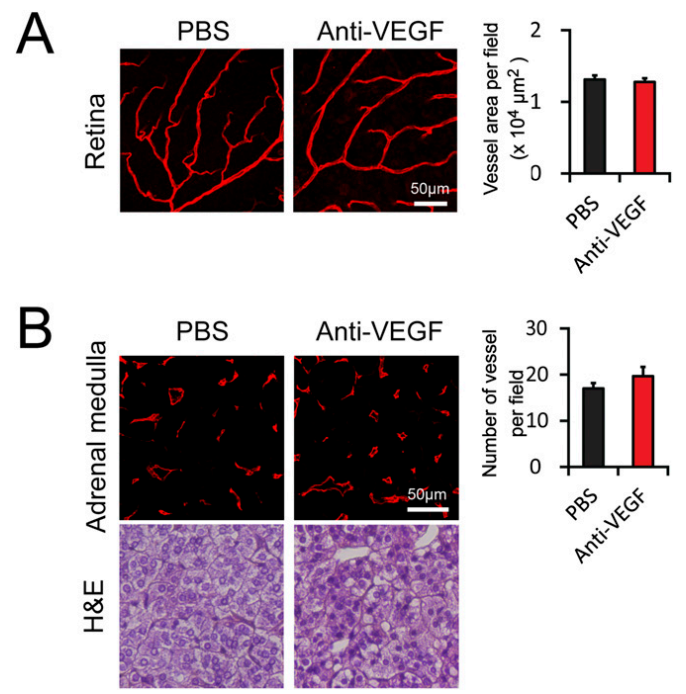

Fig. S5. Impact of anti-VEGF antibody treatment on vasculature in retina and adrenal medulla. $(A) \mathrm{CD}_{3} 1^{+}$retina microvessels (red). Vessel areas were quantified (20x magnification, $n=8$ per group). (B) Endomucin ${ }^{+}$adrenal medulla microvessels were detected in paraffin-embedded tissues (red). H\&E staining was used to reveal tissue structures. Vessel numbers were quantified ( $20 \times$ magnification, $n=8$ per group). Data are presented as means \pm SEM.
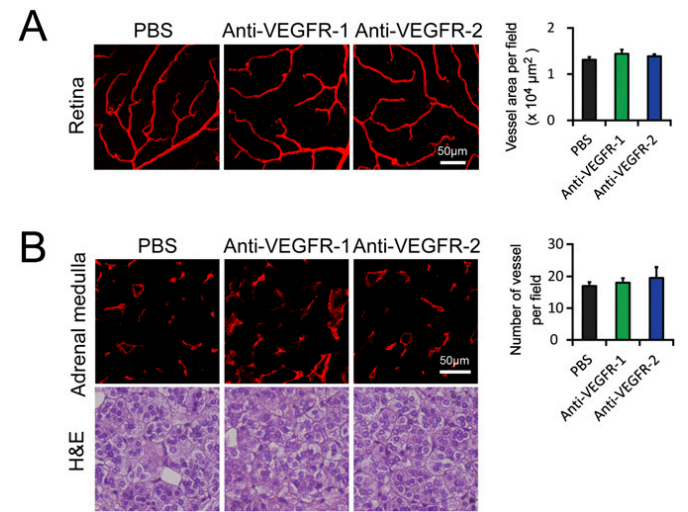

Fig. S6. Impact of anti-VEGFR-1 and anti-VEGFR-2 antibody treatment on vasculature in retina and adrenal medulla. ( $A$ ) CD31 ${ }^{+}$retina microvessels (red). Vessel areas were quantified ( $20 \times$ magnification, $n=8$ per group). ( $B$ ) Endomucin ${ }^{+}$adrenal medulla microvessels were detected in paraffin-embedded tissues (red). H\&E staining was used to reveal tissue structures. Vessel numbers were quantified ( $20 \times$ magnification, $n=8$ per group). Data are presented as means \pm SEM 




\title{
Opposing Effects of Circadian Clock Genes Bmal1 and Period2 in Regulation of VEGF-Dependent Angiogenesis in Developing Zebrafish
}

\author{
Lasse Dahl Jensen, ${ }^{1,3}$ Ziquan Cao, ${ }^{3}$ Masaki Nakamura, ${ }^{1}$ Yunlong Yang, ${ }^{1}$ Lars Bräutigam, ${ }^{2}$ Patrik Andersson, ${ }^{1}$ Yin Zhang, \\ Eric Wahlberg, ${ }^{3}$ Toste Länne, ${ }^{3}$ Kayoko Hosaka, ${ }^{1}$ and Yihai $\mathrm{Cao}{ }^{1,3, *}$ \\ ${ }^{1}$ Department of Microbiology, Tumor and Cell Biology \\ ${ }^{2}$ Department of Medical Biochemistry and Biophysics, Division of Biochemistry \\ Karolinska Institute, 17177 Stockholm, Sweden \\ ${ }^{3}$ Department of Medicine and Health Sciences, Linköping University, 58185 Linköping, Sweden \\ ${ }^{*}$ Correspondence: yihai.cao@ki.se \\ http://dx.doi.org/10.1016/j.celrep.2012.07.005
}

\section{SUMMARY}

Molecular mechanisms underlying circadianregulated physiological processes remain largely unknown. Here, we show that disruption of the circadian clock by both constant exposure to light and genetic manipulation of key genes in zebrafish led to impaired developmental angiogenesis. A bmal1-specific morpholino inhibited developmental angiogenesis in zebrafish embryos without causing obvious nonvascular phenotypes. Conversely, a period2 morpholino accelerated angiogenic vessel growth, suggesting that Bmal1 and Period2 display opposing angiogenic effects. Using a promoterreporter system consisting of various deleted vegfpromoter mutants, we show that Bmal1 directly binds to and activates the vegf promoter via E-boxes. Additionally, we provide evidence that knockdown of Bmal1 leads to impaired Notch-inhibition-induced vascular sprouting. These results shed mechanistic insight on the role of the circadian clock in regulation of developmental angiogenesis, and our findings may be reasonably extended to other types of physiological or pathological angiogenesis.

\section{INTRODUCTION}

In mammals and other vertebrates, the circadian clock plays a central role in regulation of physiological functions of almost all tissues and organs (Panda et al., 2002; Sehgal and Mignot, 2011). It is known that some crucial genes involved in regulation of cell growth, differentiation, and survival are tightly regulated by the clock genes (Hunt and Sassone-Corsi, 2007). Owing to the key importance of the circadian clock, certain transcription factors, such as Bmal1 and Clock, and transcription coregulators Period2 and Cryptochrome, or their homologs specialized for circadian regulation of targeted genes, are widely present in vertebrates, eukaryotic cells, and even prokaryotic cells (Schultz and Kay, 2003). As such, disruption of the circadian clock may lead to the onset, development, and progression of certain diseases, including neuronal disorders, metabolic disorders, chronic inflammation, and even cancer (Allada, 2003). Therefore, understanding the molecular mechanisms that control the circadian clock is pivotal for prevention and treatment of these common human diseases.

Angiogenesis is an essential process for embryonic development and for maintenance of certain physiological functions in the adult (Folkman, 1995). For example, the female reproductive cycle and wound healing are dependent on angiogensis. Under pathological conditions, uncontrolled growth of new blood vessels could lead to the onset and development of diseases. Tumor growth and metastasis are dependent on neovascularization, and targeting pathological blood vessels in tumors has been demonstrated as an effective approach for cancer therapy in human patients (Cao and Langer, 2010; Folkman, 2007; Kerbel and Folkman, 2002). Additionally, antiangiogenic therapy has produced remarkably beneficial effects on vision improvement in patients with age-related macular degeneration (Stone, 2006).

Angiogenesis is regulated by both positive and negative factors, and the expression levels of these factors can be altered under pathological conditions (Cao, 2009a, 2009b). It is generally believed that an angiogenic switch is turned on by tipping the balance of angiogenic factors and inhibitors toward domination of stimuli. Members of the vascular endothelial growth factor (VEGF) family are the most well characterized, which include VEGF (also called VEGF-A), -B, -C, -D, and placental growth factor (PIGF) (Cao, 2009a). These angiogenic factors bind to three tyrosine kinase (TK) receptors, VEGFR-1, VEGFR-2, and VEGFR-3 expressed on the surface of blood or lymphatic vessel endothelial cells to exert their biological functions (Caunt et al., 2008; Pan et al., 2007; Soker et al., 1998). It is generally believed that VEGFR-2 is the functional receptor that transduces angiogenic and vascular permeability signals in response to VEGF stimulation. In contrast to VEGFR-2, the function of VEGFR-1 remains poorly understood, and it may act as a decoy receptor for the VEGF-triggered signaling (Cao, 2009a; Eriksson et al., 2002). The DIl4-Notch signaling system has recently been reported to restrict vascular sprouting and to modulate vascular remodeling and maturation (Hellström et al., 2007; NogueraTroise et al., 2006; Ridgway et al., 2006; Siekmann and Lawson, 
2007). These functions are tightly associated with modulation of the VEGF-induced angiogenic activity. Inhibition of DII4 or the Notch receptor signaling system leads to an increased number of vascular sprouts in tumors and other tissues (Cao et al., 2008; Noguera-Troise et al., 2006).

Despite the vast knowledge of circadian biology and angiogenesis, the role of the circadian clock in regulation of angiogenesis and vascular patterning remains poorly understood. In the present study, we use developing zebrafish embryos to demonstrate the crucial role of the circadian clock in controlling vascular network formation and patterning. Our findings may imply novel therapeutic options for the treatment of angiogenesis-dependent diseases, such as cancer and cardiovascular diseases, by targeting the circadian clock.

\section{RESULTS}

Exposure to Constant Light, but Not Constant Darkness, Impairs Vascular Development

To study the impact of the circadian clock on vascular development, Tg(Fli1:egfp) zebrafish (Lawson and Weinstein, 2002) embryos at the age of $1 \mathrm{hr}$ postfertilization (hpf) were exposed to constant cold-light (LL,1800 lux), constant darkness (DD), or a $12 \mathrm{hr}$ light-dark (LD) cycle. Under identical conditions except the light source, LL-exposure neither affected the zebrafish viability nor caused any abnormal phenotypes (Figure 1A). Similarly, DD exposure did not result in any morphological changes of developing zebrafish. Notably, the somite length and the head angles, two widely used parameters for staging the development of zebrafish embryos (Kimmel et al., 1995), were virtually identical in LL- or LD-exposed zebrafish embryos (Figure 1B). These findings indicate that LL-exposure is well tolerated by zebrafish embryos. At $24 \mathrm{hpf}$ under constant light exposure, development of intersegmental vessels (ISVs) was significantly impaired relative to those embryos exposed to LD (Figure 1C). Quantification analysis showed that the average length of ISVs in LL-exposed zebrafish embryos was significantly shorter relative to those embryos exposed to LD (Figure 1D). Similar to ISVs, exposure of zebrafish embryos to LL until $72 \mathrm{hpf}$ led to significantly decreased development of subintestinal veins (SIVs, Figures $1 \mathrm{C}$ and $1 \mathrm{D}$ ). In contrast to LL, DD exposure did not result in significant alterations of ISVs or SIVs as compared to LD exposure (Figures 1C and 1D). These findings demonstrate that LL exposure significantly impairs developmental angiogenesis in zebrafish.

To study the impact of LL and DD exposure in modulation of period2, a known clock gene to be affected by light (Vatine et al., 2009), period2 expression levels were quantitatively analyzed using an RT-PCR method. As expected, period2 expression under LD conditions exhibited circadian rhythmicity, being upregulated during light exposure and downregulated during the dark phase (Figures $1 \mathrm{E}$ and $1 \mathrm{~F}$ ). Interestingly, DD exposure led to marked regression of period2 expression levels, which were barely detectable at the various hpf time points. Conversely, LL exposure resulted in persistent upregulation of period2 (Figures $1 \mathrm{E}$ and $1 \mathrm{~F}$ ). These findings demonstrate that exposure of zebrafish embryos to LL and DD could lead to differential expression of the clock gene period2.

\section{Opposing Effects of Bmal1 and Period2}

\section{on Developmental Angiogenesis}

To further delineate the role of the circadian clock in regulation of developmental angiogenesis, we utilized the morpholino technology to interrupt the circadian clock in zebrafish embryos by knocking down expression of some critical circadian genes, including bmal1, bmal2, period2, and clock3 (Allada, 2003). These morpholinos have previously been shown to impair pineal gland development (Appelbaum et al., 2005; Triqueneaux et al., 2004; Vatine et al., 2009; Ziv et al., 2005). As the pineal gland serves as a master circadian pacemaker, disruption of its development by these morpholinos would have a significant impact on the circadian clock as a whole. To demonstrate that these morpholinos sufficiently blocked translation of their corresponding clock gene products, zebrafish cDNAs coding for clock genes, including bmal1, bmal2, clock3, and period2, were cloned into a pCS2-C-myc vector, which allowed generation of fusion proteins with a myc-tag. The in vitro-transcribed mRNAs were injected with or without morpholinos into zebrafish embryos. As shown in Figure S1, bmal1, bmal2, clock3, and period2 morpholinos effectively blocked translation of injected corresponding mRNA in zebrafish embryos as detected by an anti-myc-tag antibody. These data demonstrate that the morpholinos used in our study efficiently knocked down their corresponding clock gene products.

Interestingly, injection of the bmal1 morpholino at the one-cell stage of developing zebrafish embryos under LD led to marked repression of ISV development compared to scrambled or mismatch bmal1 morpholinos (Figures 2A and 2B). Downregulation of the Bmal1 function by the specific morpholino resembled the LL-induced vascular impairment (Figure 1). Surprisingly, knockdown of Period2 under LD conditions did not suppress vascularization but unexpectedly enhanced ISV growth as compared with the mismatch morpholino control (Figures 2A and 2B). These findings suggested that Bmal1 and Period2 might display opposing effects on embryonic vessel development under the LD physiological condition. Morpholinos specifically targeting bmal 2 and clock 3 were also tested in the same experimental setting. In contrast to bmal1 and period2 morpholinos, delivery of bmal2 or clock3 morpholinos to zebrafish embryos did not significantly affect ISV development (Figures 2A and 2B). Similarly, a combination of bmal1 and bmal2 morpholinos did not produce any additive effects as compared with bmal1 morpholino alone (Figures 2A and 2B).

To gain further molecular information on the LL-induced vascular impairment in relation to circadian genes, Bmal1knock-down zebrafish embryos were exposed to LL. Intriguingly, knockdown of Bmal1 followed by LL exposure did not produce an additive effect on the impairment of ISVs, suggesting that downregulation of Bmal1 mediates LL-induced vascular retardation (Figures 2A-2C). Conversely, knockdown of Period2 under LL exposure completely rescued the $\mathrm{LL}$-induced vascular defects on ISV development (Figures $2 \mathrm{~A}-2 \mathrm{C}$ ), validating the negative role of Period2 in regulation of vascular development. Indeed, quantitative real-time PCR (qPCR) analysis demonstrated that period2 was markedly upregulated at 48 and $72 \mathrm{hpf}$ under LL exposure (Figure 2l). Taken together, these findings demonstrate that Bmal1 acts as a positive mediator 

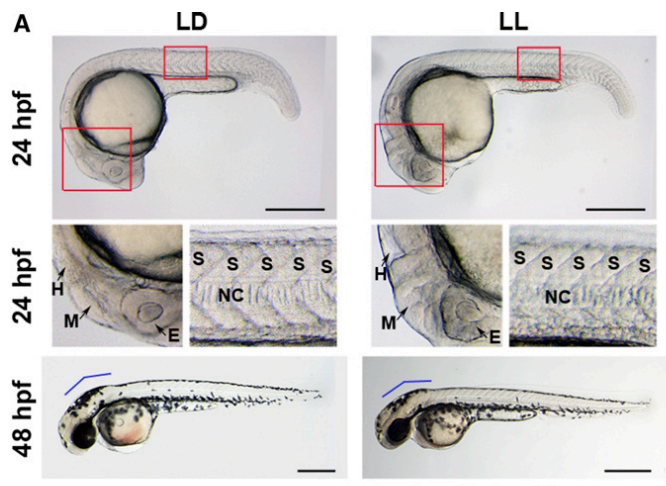

B

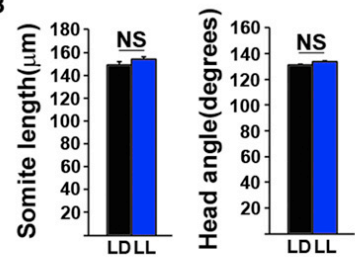

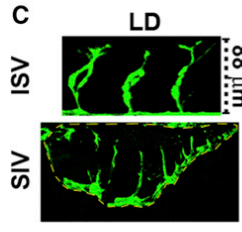

DD

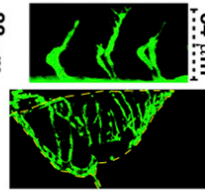

LL

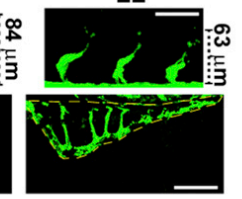

D

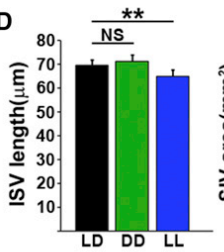

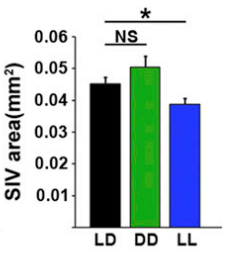

E

P2 \begin{tabular}{lllllllll}
\hline 24 & 30 & 36 & 42 & 48 & 54 & 60 & 66 & 72
\end{tabular} LD DD

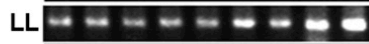

F

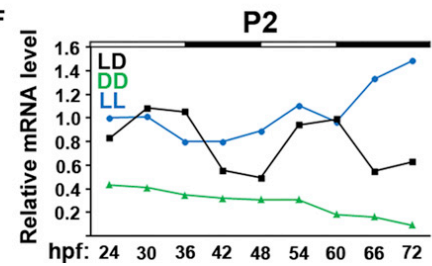

Figure 1. Constant Light Exposure Inhibits Developmental Angiogenesis

$T g(F l i 1: E G F P)^{y 1}$ transgenic zebrafish embryos exposed to LD (A-D), LL (A-D), or DD (C and D) were examined for ISV development at $24 \mathrm{hpf}(\mathrm{C}$ and D) or for SIV development at $72 \mathrm{hpf}(\mathrm{C}$ and $\mathrm{D})$ using confocal microscopy.

(A) Morphology of zebrafish embryos was examined at $24 \mathrm{hpf}$ and $48 \mathrm{hpf}$ under LD and LL conditions.

(B) Average \pm SEM somite lengths and head angles were quantified under LD and LL conditions ( $n=7-9$ /group).

(C) Zebrafish embryos were raised under LD, DD, or LL conditions from $1 \mathrm{hpf}$. Dashed lines indicate the maximal length of ISVs. Yellow dashed lines encircle the SIV. Scale bar for ISV is $50 \mu \mathrm{m}$ and for SIV is $100 \mu \mathrm{m}$.

(D) Quantification of the average \pm SEM. ISV length ( $n=52-54$ embryos/group) of $24 \mathrm{hpf}$ embryos and the SIV area of 72 hpf embryos $(n=48$ embryos).

(E) RT-PCR analysis of average zebrafish period2 (P2) expression \pm SEM under LD, LL, and DD conditions (a pooled sample of 25 zebrafish embryos/group). (F) Densitometric quantification of average period2 expressions shown in (E).

Bars in $(A)=500 \mu \mathrm{m}$. S, somite; $N C$, notochord; $H$, hindbrain; M, mesencephalon; $E$, eye. Blue lines in $(A)$ indicate the head angle. ${ }^{*} p<0.05$ and ${ }^{* *} p<0.01$. NS, not significant.

See also Figure S2.

for vascular development, whereas Period2 negatively regulates angiogenesis.

Similar to LL exposure, neither bmal1 nor period2 morpholinos caused nonvascular defects, including changes in the somite development and the head angles (Figures 2D-2E). The vascular specific effects of bmal1 and period2 morpholinos were further validated using other specific organ or tissues markers. In situ hybridization showed that expression of sonic hedgehog (shh), a key morphogen during organogenesis and expression of acetylated alpha tubulin, a marker for axon maturation and stability was not altered (Figure S2).

Restoration of LL- or bmal1 Morpholino-Induced ISV Defects by bmal1 mRNA

To further validate the role of Bmal1 and Period2 in modulation of ISV development, we performed gain-of-function experiments by injection of zebrafish bmal1 or period 2 mRNA under various conditions. As expected, injection of bmal1 mRNA resulted in 


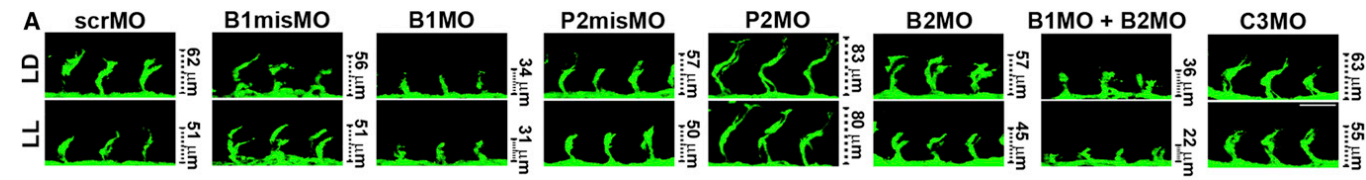

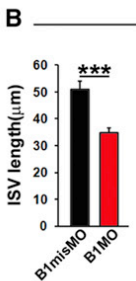

D

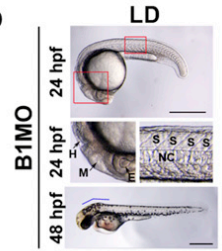

LD

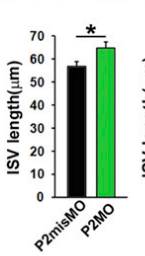

LL

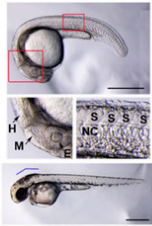

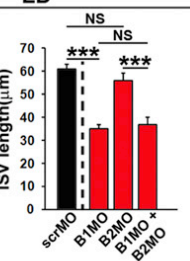

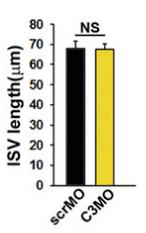

LD

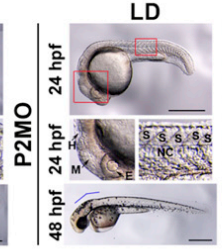

C

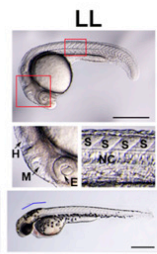

E

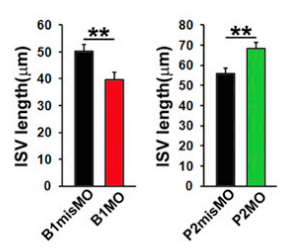

L

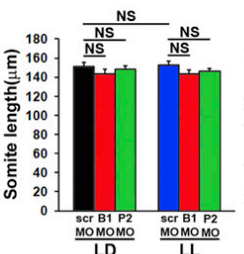

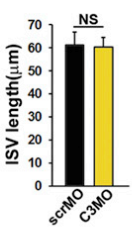

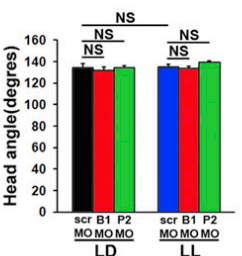

$\mathbf{F}$
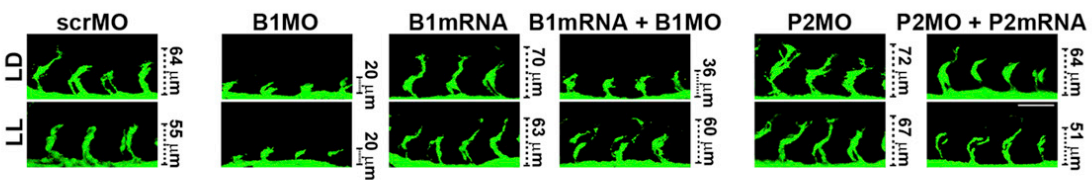

G

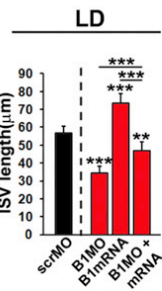

H

LL

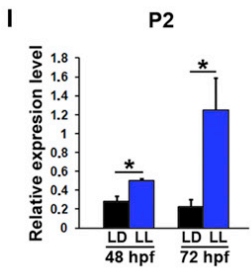

Figure 2. Opposing Roles of Bmal1 and Period2 in Regulation of Developmental Angiogenesis

(A) Zebrafish embryos received injections of specific morpholinos targeting Bmal1 (B1MO), Bmal2 (B2MO), Clock3 (C3MO), or Period2 (P2MO) (0.2 pmol/embryo) and were subsequently raised under LL or LD conditions. Scrambled morpholino (scrMO), five base-pair mismatch bmal1 (B1misMO), or five base-pair mismatch period2 ( $\mathrm{P} 2$ misMO) were used as controls. Dashed lines indicate the maximal length of ISVs. Bar $=50 \mu \mathrm{m}$.

(B) Quantification of the average length \pm SEM of ISVs in $24 \mathrm{hpf}$ embryos under the LD condition ( $\mathrm{n}=8-48$ embryos/group).

(C) Quantification of the average length \pm SEM of ISVs in 24 hpf embryos under the LL condition ( $n=6-37$ embryos/group).

(D) Morphology of zebrafish embryos was examined at $24 \mathrm{hpf}$ and $48 \mathrm{hpf}$ under LD and LL conditions. Bars $=500 \mu \mathrm{m}$. S, somite; NC, notochord; H, hindbrain;

$\mathrm{M}$, mesencephalon; E, eye.

(E) Quantification of average \pm SEM somite lengths and head angles under LD and LL conditions ( $\mathrm{n}=7-17 / \mathrm{group})$.

(F) Zebrafish embryos received injections of specific morpholinos targeting Bmal1 (B1MO), Period2 (P2MO), scrambled morpholino (scrMO), bmal1 mRNA (B1mRNA), period2 mRNA (P2mRNA), B1mRNA plus B1MO, or P2mRNA plus P2MO and were subsequently raised under LD or LL conditions. Dashed lines indicate the maximal length of ISVs. Bar $=50 \mu \mathrm{m}$

(G) Quantification of the average length \pm SEM of ISVs in 24 hpf embryos under the LD condition ( $n=8-27$ embryos/group).

(H) Quantification of the average length \pm SEM of ISVs in $24 \mathrm{hpf}$ embryos under the LL condition ( $\mathrm{n}=6-28$ embryos/group).

(I) qPCR of average \pm SEM period 2 mRNA expression levels after 48 or $72 \mathrm{hpf} \mathrm{LD} \mathrm{or} \mathrm{LL} \mathrm{exposure}\left(\mathrm{n}=3-4 \times 20-50\right.$ embryos/group). ${ }^{*} \mathrm{p}<0.05$; ${ }^{* *} \mathrm{p}<0.01$; and ${ }^{\star \star \star} \mathrm{p}<0.001$. NS, not significant.

See also Figures S1 and S2. 
a significant increase of ISV length (Figures $2 \mathrm{~F}-2 \mathrm{H}$ ). Intriguingly, sequential injection of bmal1 morpholino followed by bmal1 mRNA significantly restored ISV development (Figures $2 \mathrm{~F}$ and 2G). Similarly, delivery of bmal1 mRNA completely rescued the LL-induced ISV defects (Figures 2F-2H). Conversely, administration of period $2 \mathrm{mRNA}$ significantly antagonized the period2 morpholino-induced vessel growth (Figures $2 \mathrm{~F}$ and $2 \mathrm{H}$ ). These findings demonstrate that the morpholinos used in our study were specific for bmal1 and period2 and further validate the vascular role of these clock genes.

\section{VEGF Mediates Bmal1-Induced Angiogenesis}

VEGF is a key angiogenic factor during embryonic development and in pathological conditions, including tumor growth and ophthalmological disorders (Ferrara et al., 2003; Nagy et al., 2007). In mice, heterozygous deletion of the vegf gene leads to early embryonic lethality owing to lack of vascular and hematopoietic development, demonstrating that an optimal level of VEGF is essential to ensure appropriate development of various tissues and organs (Carmeliet et al., 1996; Ferrara et al., 1996). To study the involvement of VEGF and its receptor signaling system in mediating circadian-modulated angiogenesis, a specific vegf morpholino (Nasevicius et al., 2000) was used in combination with bmal1 or period 2 morpholinos. As expected, delivery of a high dose of vegf morpholino ( $0.2 \mathrm{pmol} / \mathrm{embryo})$ to zebrafish embryos resulted in virtually complete suppression of ISV growth at $24 \mathrm{hpf}$ (Figure 3B), suggesting that VEGF is absolutely required for ISV development. However, injection of a low dose of vegf morpholino ( $0.06 \mathrm{pmol} / \mathrm{embryo})$ led to an only partial but statistically significant inhibition of ISV development (Figures 3A and 3C). Interestingly, a combination of vegf and bmal1 morpholinos additively inhibited ISV growth, whereas coinjection of vegf and period2 morpholinos did not result in an additional inhibitory effect but a slightly increased angiogenic response (Figures $3 \mathrm{~A}$ and $3 \mathrm{C}$ ).

Similar to the vegf morpholino, addition of an orally active VEGFR tyrosine kinase inhibitor (ZN323881, TKI) (Cao et al., 2008 ) to the aquarium water at a low concentration resulted in partial suppression of ISV development (Figures $3 \mathrm{~A}$ and $3 \mathrm{C}$ ). Again, delivery of a combination of ZN323881 and the bmal1 morpholino resulted in an additive effect on suppression of the ISV growth (Figures 3A and $3 \mathrm{C}$ ). Conversely, a combination of ZN323881 and period2 did not further potentiate the inhibitory activity as compared with ZN323881 alone. An independent experiment using sunitinib, another orally active VEGFR TKI, produced virtually identical results (Figure S3).

\section{Bmal1 Targets the vegf Promoter for Transcriptional Activation}

To provide direct evidence that the vegf gene is a direct target for Bmal1, a vegf promoter-luciferase reporter system was used to study regulation of vegf promoter activity by the circadian clock in zebrafish embryos. A bmal1 promoter-luciferase reporter system was also constructed to measure alteration of the Bmal1 level by the circadian clock. We used both human and zebrafish vegf promoter-luciferase in our studies. Intriguingly, an almost perfect coordination of expression patterns was observed between human VEGF and mouse Bmal1 under LD exposure (Figure 3G), suggesting that VEGF expression levels are modulated by the circadian clock. Strikingly, the correlation between bmal1 and vegf promoter activity was disrupted under LL conditions (Figure 3G). Inversely, bmal1 and vegf promoter activity coincided and exhibited circadian rhythmicity under DD conditions (Figure 3G).

Similarly, zebrafish vegf promoter activity was also coordinately regulated by the circadian clock under LD and DD conditions (Figure $3 \mathrm{H}$ ), and LL-exposure significantly suppressed zebrafish vegf promoter activity (Figure 3D). To validate these findings, we measured the endogenous levels of bmal1 and vegf mRNA in zebrafish using a QPCR method. In principle, the circadian expression pattern of bmal1 matched that of vegf under LD exposure (Figure 3I). Particularly, endogenous expression levels of zebrafish bmal 1 and vegf exhibited coordinated rhythmicity even during prolonged LD exposure (Figures S4A and $\mathrm{S} 4 \mathrm{~B}$ ). These findings provide compelling evidence that VEGF coincides with Bmal1 expression under LD conditions. Again, LL exposure almost completely ablated the rhythmic expression of vegf and bmal1 genes (Figure 3l), indicating that expression levels of bmal1 and vegf are tightly regulated by the circadian clock. Similar to LD conditions, zebrafish embryos under DD exposure retained the rhythmicity of bmal1 and vegf expression (Figure 3l).

As an independent proof of the intimate interaction between Bmal1 and VEGF expression, knockdown of Bmal1 led to significantly decreased levels of human and zebrafish VEGF expression (Figures $3 \mathrm{E}$ and $3 \mathrm{~F}$ ), demonstrating that Bmal1 is required to maintain a steady-state level of VEGF expression. These findings provide a mechanistic insight that Bmal1 might directly target the vegf promoter for transcriptional regulation.

To study if Bmal1 directly targets the vegf promoter, we analyzed the promoter regions of the vegf genes in zebrafish, mice, and humans. In zebrafish, we identified four confirmed E-box regions that potentially bind to the Bmal1-transcription complex (Figures $3 \mathrm{~J}$ and $3 \mathrm{~K}$ ). There were totally five such Bmal1-binding E-boxes on the promoter region of the mouse vegf gene and one exist in the promoter region of the human vegf gene (Figure $3 \mathrm{~J}$ ). In addition, multiple unconfirmed Bmal1binding E-boxes exist in all three species and these E-box sites are likely to bind to Bmal1. To study if E-boxes in the zebrafish VEGF promoter are involved in Bmal1-regulated VEGF expression, a series of E-box-deleted mutants of the promoter was generated (Figure 3K). Deletion of E-box 1 resulted in significant reduction of the zvegf promoter activity (Figures $3 \mathrm{~K}$ and $3 \mathrm{~L}$ ). Similarly, deletion of the cluster E-boxes $2-4$ virtually completely abolished the zvegf promoter activity (Figures $3 \mathrm{~K}$ and $3 \mathrm{~L}$ ). These findings demonstrate that E-boxes in the zvegf promoter are essentially required for transcriptional regulation of VEGF expression. We next performed a chromatin immunoprecipitation assay in which an anti-mouse Bmal1 antibody was used to precipitate Bmal1 protein using a mouse cell line. The crosslinked DNA-protein complexes were then disassociated and PCR amplified using several pairs of specific primers covering various regions of the vegf promoter. An E-box-less vegf fragment was used as a negative control, and a fragment containing the first two E-boxes in the period1 gene was used as a positive control as previously described (Jung et al., 2003). As expected, 

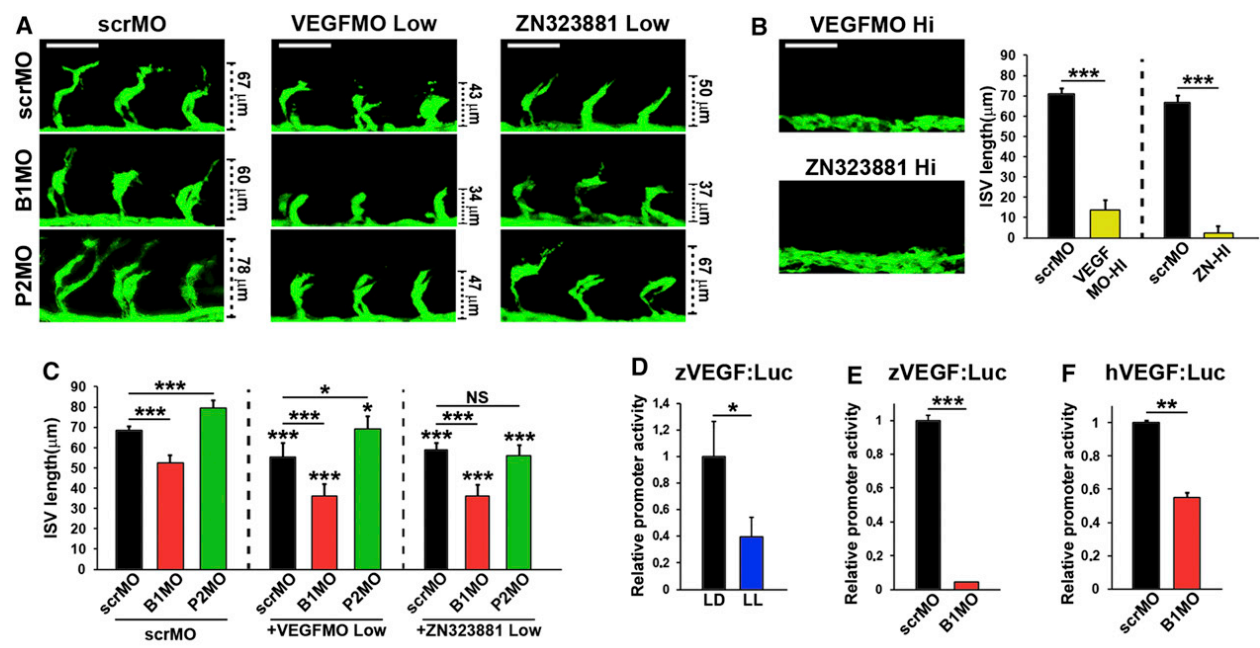

G
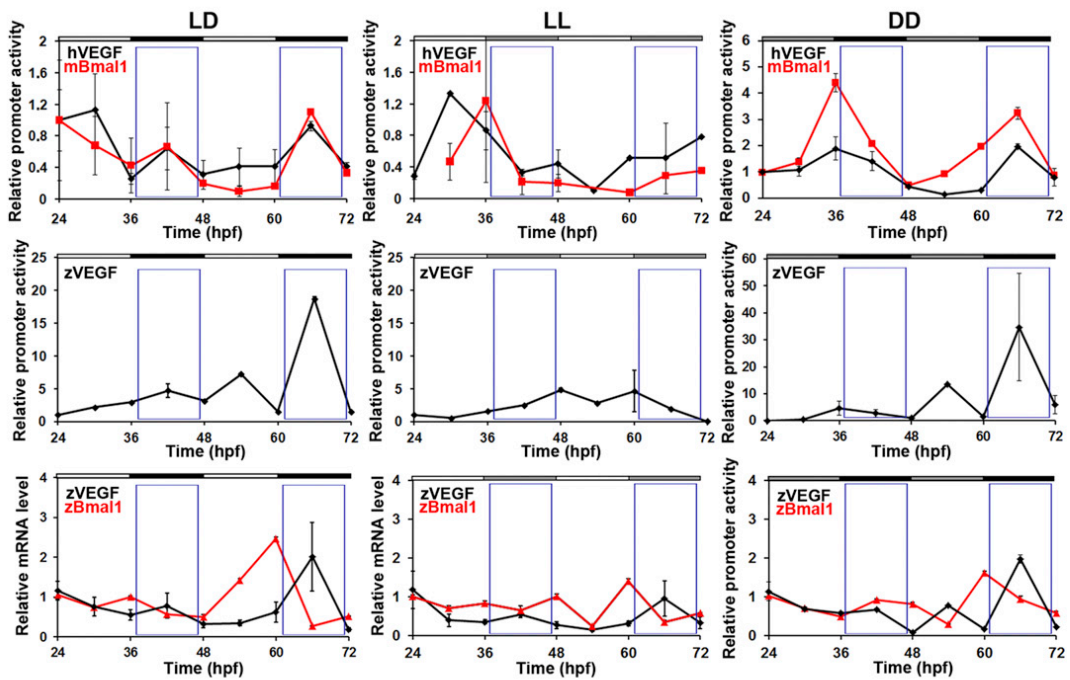

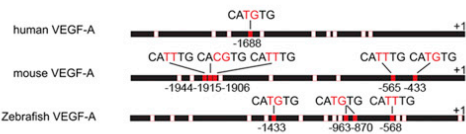

K

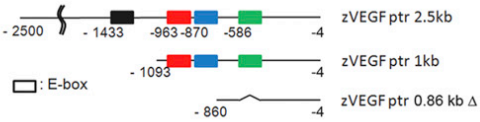

L

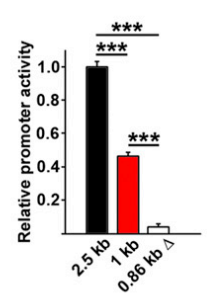

M

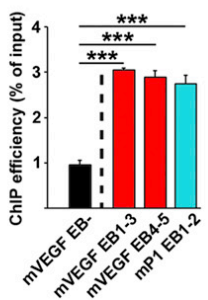


fragments containing E-boxes $1-3$ and $4-5$ in the vegf promoter region showed direct binding to Bmal1 (Figure $3 \mathrm{M}$ ), whereas the E-box-less fragment did not show significant binding activity. Taken together, these results provide compelling evidence that Bmal1 directly binds to the vegf promoter via E-boxes.

\section{Relation of Circadian and Notch Signaling in Regulation of Vessel Development}

To study the involvement of the Notch signaling system in modulation of the circadian clock-VEGF axis, three independent approaches were used to block the Notch pathway. First, a $\gamma$-secretase inhibitor (DAPT) that inhibits the Notch signaling system was used. As expected, addition of DAPT to the aquarium water resulted in the formation of high-density vascular networks and a high number of vascular braches from ISVs (Figures 4A and 4B). Second, we designed a morpholino specifically targeting recombining binding protein suppressor of hairless (rbpsuh) (Siekmann and Lawson, 2007) that mediates the Notch signaling. Similar to DAPT, delivery of the rbpsuh morpholino also led to the formation of a disorganized ISV network that consisted of a high density of microvessels that grew in widely different directions (Figures $4 \mathrm{~A}$ and $4 \mathrm{~B}$ ). Finally, a genetic mutant strain of zebrafish that lacks functional mind-bomb, a ubiquitin ligase involved in mediating the Notch signaling (Haddon et al., 1998), was used. Again, a high degree of ISV malformation and excessive vascular braches/sprouts existed in the mind-bomb mutant zebrafish (Figures 4A and 4B). These findings provide compelling evidence that inhibition of the Notch signaling system leads to the formation of a high density of disorganized vascular networks in the ISV region.

Interestingly, delivery of the bmal1 morpholino to zebrafish embryos markedly reduced the Notch inhibition-induced vascular malformation and excessive vascular sprouts (Figures $4 \mathrm{~A}$ and $4 \mathrm{~B}$ ). Inversely, the period2 morpholino did not significantly affect the Notch inhibition-induced vascular phenotype
(Figures 4A and 4B). Administration of the vegf morpholino at a low dose to zebrafish embryos partly prevented the Notch inhibition-induced vascular phenotypes (Figures 4A and 4B), suggesting that VEGF mediates the Notch-induced vascular changes. As described above, expression levels of VEGF and Bmal1 were downregulated under the LL condition. Under LL exposure, the Notch inhibition-induced vascular malformation was partially prevented (Figures $4 \mathrm{C}$ and $4 \mathrm{D}$ ). On the contrary, knockdown of Period2 under LL further potentiated the Notch inhibition-induced vascular phenotype (Figures 4C and 4D). These findings suggest that the circadian clock-regulated VEGF expression levels act as a driving force for the Notchinduced vascular changes.

\section{DISCUSSION}

The circadian clock controls growth, migration, differentiation, and survival of many cell types and is involved in regulation of physiological and pathological processes, such as embryonic and disease development. Angiogenesis is required for embryonic development and excessive or defective blood vessel growth has been linked to various human diseases (Cao, 2009a; Cao et al., 2011). However, the role of the circadian clock in regulation of angiogenesis under physiological and pathological conditions remains poorly understood. Using zebrafish as an in vivo model, we, in the present study, provide compelling evidence that developmental angiogenesis is tightly controlled by the circadian clock. At the molecular level, we showed that Bmal1 and Period2 displayed opposing effects on vascular development via regulation of VEGF expression. The circadian clock-regulated VEGF levels crosstalk with the Notch signaling system in controlling the extent of vessel sprouts and functions. Although the study was performed in zebrafish, our data may be reasonably extended to mammals, including humans because the clock genes are highly conserved among these species.

Figure 3. Circadian Clock Regulates Expression Levels of VEGF

(A) Zebrafish embryos were injected with scrambled (scrMO) morpholino (A) or a low dose of the vegf morpholino (VEGFMO, 0.06 pmol/embryo) alone or in combinations with bmal1 (B1MO) or period2 (P2MO) morpholinos (0.2 pmol/embryo). Some embryos were treated with either DMSO vehicle or a low dose of VEGFR TKI $(0.3 \mu \mathrm{M}$ ZN323881) alone or in combinations with bmal1, period 2 , or scrambled morpholinos $(0.2$ pmol/embryo). The embryos were subsequently raised under the $L D$ condition.

(B) High doses of the vegf morpholino (0.2 pmol/embryo) or ZN323881 (1.0 $\mu \mathrm{M})$ were also delivered to zebrafish embryos. Dashed lines in (A and B) indicate the maximal length of ISVs in various groups. Bars $=50 \mu \mathrm{m}$. Quantification of the average ISV length \pm SEM in $24 \mathrm{hpf}$ embryos ( $\mathrm{n}=12-68 \mathrm{embryos} / \mathrm{group}$ ).

(C) Quantification of the average ISV length \pm SEM in $24 \mathrm{hpf}$ embryos from the experiment shown in (A) (average $\mathrm{n}=36 \mathrm{embryos} / \mathrm{group}$ ).

(D) Zebrafish vegf promoter activity as measured by average luciferase activity \pm SEM in zebrafish embryos exposed to LD or LL ( $n=20$ embryos/group).

(E) Zebrafish vegf promoter activity in scrambled or bmal1 morpholino-treated zebrafish embryos as measured by average luciferase activity \pm SEM $(n=20$ embryos/group).

(F) Human vegf promoter activity in scrambled or bmal1 morpholino-treated zebrafish embryos as measured by average luciferase activity \pm SEM ( $=20$ embryos/group).

$(\mathrm{G}-\mathrm{H})$ Values of average relative luciferase activity \pm SEM in embryos exposed to LD, LL, or DD. Black line: Luciferase activity in zebrafish embryos injected with human vegf promoter-luciferase $(\mathrm{G})$ or zebrafish vegf promoter-luciferase $(\mathrm{H})$. Red lines in $\mathrm{G}$ represent luciferase activity in zebrafish embryos injected with a mouse bmal1 promoter-luciferase reporter plasmid.

(I) qPCR analysis of average \pm SEM endogenous levels of bmal1 (red line) and vegf (black line) under LD, LL, and DD conditions.

(J) Sequence analysis of human, mouse, and zebrafish vegf promoter regions that contain E-boxes that bind to Bmal1. Red boxes represent the known Bmal1 binding E-boxes, and white boxes represent unknown binding E-boxes.

(K) Schematic diagram showing various deleted mutants of the zebrafish vegf promoter region. ptr, promoter.

(L) Average luciferase activity \pm SEM in embryos injected with various deleted mutants of the zebrafish vegf promoter-luciferase and subsequently raised under LD exposure ( $\mathrm{n}=20$ embryos/group).

(M) ChIP assay of average Bmal1 binding \pm SEM to the zebrafish vegf promoter. EB, E-box. ${ }^{\star} \mathrm{p}<0.05 ;{ }^{* \star} \mathrm{p}<0.01$; and ${ }^{* \star *} \mathrm{p}<0.001$. NS, not significant. See also Figures S3 and S4. 


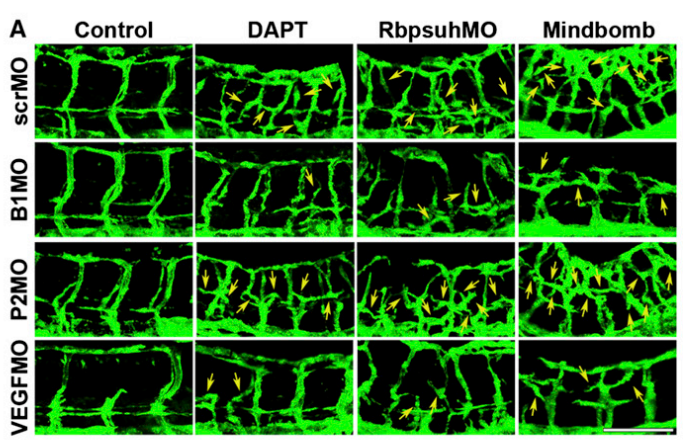

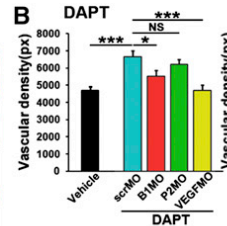
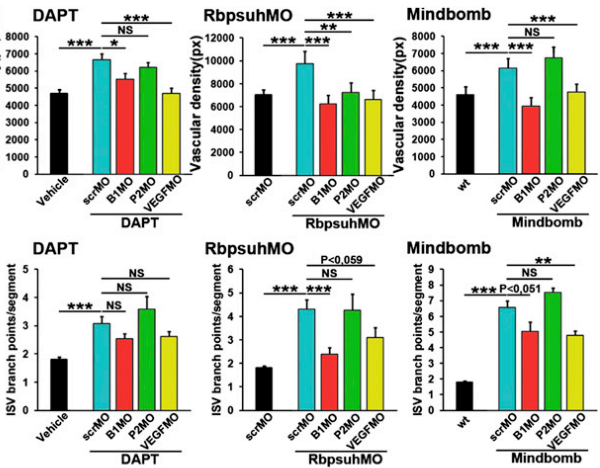

D
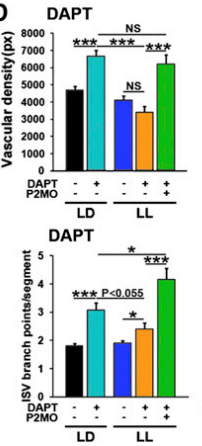
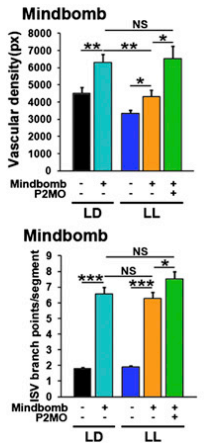

Figure 4. Involvement of the Notch Signaling Pathway in Circadian Clock-Regulated Vascular Sprouting

(A) Zebrafish embryos received bmal1 (B1MO, 0.2 pmol/embryo), period2 (P2MO, 0.2 pmol/embryo) or vegf (VEGFMO, 0.1 pmol/embryo) morpholinos, either alone or in combination with DAPT $(100 \mu \mathrm{M})$, rbpsuh morpholino (RbpsuhMO, $0.1 \mathrm{pmol} / \mathrm{embryo})$, or in the mind-bomb background. The embryos were subsequently raised under the LD condition. Arrows point to ISV braches in each group. Scale bar is $100 \mu \mathrm{m}$.

(B) Quantification of the average \pm SEM. ISV density or number of branches/segment of $72 \mathrm{hpf}$ embryos in (A) (average $\mathrm{n}=23 \mathrm{embryos} / \mathrm{group}$ ).

(C) Zebrafish embryos received period2 (P2MO, 0.2 pmol/embryo) alone or in combination with DAPT (100 $\mu$ M), rbpsuh morpholino (RbpsuhMO, $0.1 \mathrm{pmol} / \mathrm{embryo}$ ), or in the mind-bomb background. The embryos were subsequently raised under LD or LL conditions. Arrows point to ISV braches in each group. Scale bar is $100 \mu \mathrm{m}$.

(D) Quantification of the average \pm SEM. ISV density or number of branches/segment of 72 hpf embryos in (C) (average $n=23$ embryos/group). ${ }^{*} p<0.05$; ${ }^{\star \star} \mathrm{p}<0.001$; and ${ }^{\star \star \star} \mathrm{p}<0.001$. NS, not significant.

During embryogenesis, vascular development is tightly regulated by various positive and negative regulators. Optimal levels of these vascular modulators are essential to ensure the formation of healthy and functional vascular networks that perfuse sufficient $\mathrm{O}_{2}$ and nutrients for tissue and organ development (Carmeliet et al., 1996; Ferrara et al., 1996; Gale et al., 2004). Inadequate expression levels of key angiogenic factors might lead to the malformation of blood vessels, which eventually terminates development of the entire embryo. For example, genetic deletion of only one allele of the vegf gene or the dll4 gene in mice (haploinsufficiency) leads to early embryonic death owing to vascular defects (Carmeliet et al., 1996; Ferrara et al., 1996; Gale et al., 2004). These studies suggest that an optimal level of VEGF is absolutely required for proper vascular development, navigation of vessel outgrowth, and maintenance of vascular functions. The VEGF expression level can be modulated by multiple factors in tissue microenvironments. For example, tissue hypoxia potently upregulates VEGF expression (Boutin et al., 2008; Makino et al., 2001). In this study, we have shown that the circadian clock directly modulates VEGF expression levels via transcriptional regulation during embryonic development. First, we show that VEGF expression in developing embryos exhibits a clear circadian rhythm. Second, the VEGF expression pattern almost completely matches the expression pattern of Bmal1, which is an essential component of the circadian clock-induced gene expression program. Third, we provide molecular evidence that the vegf promoter contains several Bmal1 binding E-boxes and that Bmal1 directly binds to the vegf promoter via these E-boxes. Finally, we showed that knockdown of Bmal1 directly reduced the vegf promoter activity using a luciferase reporter system in whole zebrafish embryos. These data provide convincing evidence that the 
circadian clock directly regulates VEGF expression and vascular development.

One of our intriguing findings is that $L L$ and $D D$ exposure leads to differential regulation of some clock genes, although both conditions are known to disrupt the circadian clock. This interesting finding is discussed in more detail in the Extended Discussion.

The implication of our findings might be extended to regulation of angiogenesis under pathological conditions. For example, it is known that disruption of the circadian clock may increase cancer incidence (Hrushesky et al., 1999; Stevens et al., 2011). It is plausible that the circadian clock-regulated VEGF expression and vascular development might significantly contribute to tumor development. This possibility warrants further investigation. Another interesting finding of our study is the relation between the circadian clock-modulated VEGF levels and the Notch signaling-induced vascular remodeling. Notch signaling restricts vascular sprouts by limiting endothelial tip cell formation and maintaining arterial identity, which is dependent on its relation to VEGF (Noguera-Troise et al., 2006; Ridgway et al., 2006). VEGF is the driving force of tip cell formation, vascular sprouting and the formation of primitive vascular networks. We showed that downregulation of VEGF expression by interruption of the circadian clock or knocking down Bmal1 leads to an abridged effect of Notch inhibition-induced vascular malformation. Interestingly, inhibition of NADPH oxidases is associated with downregulation of DII4 and Notch signaling (Bhandarkar et al., 2009). This signaling system in relation to the circadian clock warrants further study.

Despite vascular defects under circadian clock-disrupted conditions, zebrafish development was not significantly affected. Unlike mammals, zebrafish embryos undergo persistent development for approximately 1 week in the absence of vasculature or circulation (Stainier et al., 1995). These avascular zebrafish embryos are dependent on free diffusion of oxygen and nutrients. Also, compensatory mechanisms may exist in zebrafish because of the many clock gene homologs. For example, three Bmal, three Clock, four Period, and six Cryptochrome homologs have been reported to exist in zebrafish (Wang, 2008a, 2008b, 2009), and they may have redundant and distinct functions. In the present study, we provide an example of opposing vascular functions of Bmal1 and Period2. Unlike Bmal1 and Period2, knockdown of Bmal2 or Clock3 did not cause any obvious phenotypes. Additionally, several other clock gene products, including Clock1, Clock2, and Period3, were investigated using the morpholino approach. The phenotypes of Clock1-, Clock2-, and Period3-deficient zebrafish embryos are under investigation.

Taken together, our data provide mechanistic insight on circadian clock-regulated vascular development. Maintenance of the normal circadian clock would be essential to ensure proper development of vasculatures in the embryos and probably in other physiological and pathological conditions.

\section{EXPERIMENTAL PROCEDURES}

Zebrafish Strains and Husbandry

Zebrafish of the $\operatorname{Tg}(\text { fli1:EGFP })^{y 1}$ and $\operatorname{Tg}\left(\text { Mib }^{\text {ta52b/ta52b }} \text {;fli1:EGFP }\right)^{y 1}$ strains (ZFIN, Eugene, OR, USA) were used in this study, as previously described
(Cao et al., 2010; Rouhi et al., 2010). All experiments were performed in accordance with ethical permissions granted by the North Stockholm Experimental Animal Ethical Committee, Sweden.

Morpholino Treatment

All morpholinos used in this study were purchased from Gene Tools (Philomath, OR, USA).

\section{Assessment of Morpholino Efficiency}

Amplified fragments of the bmal1a, bmal2, period2, and clock3 open reading frames were cloned into the pCS2-C-Myc vector to generate C-terminal myc-tag fusion constructs. Myc-fusion proteins were detected in the presence or absence of morpholino by immunohistochemistry with a 9E10 monoclonal anti-myc antibody (Sigma-Aldrich, St. Louis, MO, USA).

\section{RT-PCR}

Total RNA was reverse-transcribed using a RevertAid $\mathrm{H}$ minus First Strand cDNA Synthesis Kit (Fermentas, Glen Burnie, MD, USA). RT-PCR was performed using DreamTaq Green PCR Master Mix (Thermo Scientific, Waltham, MA, USA). See the Extended Experimental Procedures for a list of primers used in this study. Bands were quantified by the densitometry function of the Quantity One software.

Zebrafish VEGF-Luciferase Construction and Promoter Deletion Promoter fragments of different sizes for the zebrafish vegfab gene was generated by PCR from zebrafish genomic DNA and cloned into the pGL3-Basic vector (Promega, Madison, WI, USA).

\section{Promoter Activity Assay}

Zebrafish embryos were injected with human vegf promoter-luciferase, mouse bmal1 promoter-luciferase, or zebrafish vegfab promoter-luciferase, followed by immediate transfer to LD, LL, or DD conditions. The dual luciferase kit (Vector Laboratories, Burlingame, CA, USA) was used for detection of luciferase activity.

\section{Quantitative Real-Time PCR}

RNA purification was performed from whole embryos using a GeneJET RNA purification kit (ThermoFisher Scientific). RNA levels of zebrafish genes were detected by qPCR, as described previously (Xue et al., 2008).

Generation of Capped mRNA

Capped mRNA was prepared using the mMessage/Machine Kit (Ambion, Austin, TX, USA). The full-length EST clone of Bmal1 (IMAGE 2643691) was subcloned into pCS2+.

\section{Chromatin Immunoprecipitation Assay}

Mouse fibroblast cells were used for the EZ-ChIP chromatin immunoprecipitation assay (Millipore, Billerica, MA, USA). A rabbit anti-Bmal1 antibody (2.5 $\mu \mathrm{g}$ per sample; Abcam, Cambridge, UK) and a nonimmune rabbit IgG ( $2.5 \mu \mathrm{g}$ per sample; Millipore) were used for immunoprecipitation. The purified DNA was used for qPCR analysis. See the Extended Experimental Procedures for a list of primers used.

\section{Zebrafish Histology}

Zebrafish immunohistochemistry and in situ hybridization was performed according to published protocols (Bräutigam et al., 2011) using antiacetylated tubulin antibodies (Sigma-Aldrich cat no. T-6793; 1:1,000 dilution) and a cDNA EST clone coding for zebrafish sonic hedgehog (Imagenes, SHH: IMAGE ID 9037826). Stained zebrafish were analyzed under a Leica MZ16 microscope equipped with a Leica DFC300FX camera.

\section{Microscopy and Imaging Analysis}

Zebrafish embryos were whole-mounted in VectaShield (Vector) and examined under a confocal microscope (Nikon D-Eclipse C1), as previously described (Dahl Ejby Jensen et al., 2009; Lee et al., 2009). 
Statistical Analysis

Statistical analyses were done using the standard two-tailed Student $t$ test, and ${ }^{\star} p<0.05,{ }^{\star \star} p<0.01$, and ${ }^{\star \star *} p<0.001$ were considered statistically significant. The $\mathrm{n}$ values indicate the number of individual embryos analyzed. Data were presented as means \pm SEM.

\section{SUPPLEMENTAL INFORMATION}

Supplemental Information includes an Extended Discussion, Extended Experimental Procedures, and four figures and can be found with this article online at http://dx.doi.org/10.1016/j.celrep.2012.07.005.

\section{LICENSING INFORMATION}

This is an open-access article distributed under the terms of the Creative Commons Attribution-Noncommercial-No Derivative Works 3.0 Unported License (CC-BY-NC-ND; http://creativecommons.org/licenses/by-nc-nd/3.0/ legalcode).

\section{ACKNOWLEDGMENTS}

We thank Sharon Lim for the artwork, Dr. Kazuhiro Yagita (Nagoya University) for providing a plasmid containing the mouse bmal1 promoter-luciferase construct, Dr. Yoav Gothilf (Tel Aviv University) for the full-length period2 plasmid, and Dr. José Bessa (Centro Andaluz del Biologia del Desarrollo) for the pCS2-C-Myc plasmid. Y.C.'s laboratory is supported through research grants from the Swedish Research Council, the Swedish Cancer Foundation, the Karolinska Institute Foundation, the Karolinska Institute distinguished professor award, the Tianjin Natural Science Foundation (CMM-Tianjin, no. 09ZCZDSF04400) for international collaboration between Tianjin Medical University and Karolinska Institutet, the Torsten Soderbergs Foundation, the European Union Integrated Project of Metoxia (project no. 222741), and the European Research Council (ERC) advanced grant ANGIOFAT (project no. 250021).

Received: August 19, 2011

Revised: February 24, 2012

Accepted: July 15, 2012

Published online: August 9, 2012

\section{REFERENCES}

Allada, R. (2003). Circadian clocks: a tale of two feedback loops. Cell 112, 284-286.

Appelbaum, L., Anzulovich, A., Baler, R., and Gothilf, Y. (2005). Homeoboxclock protein interaction in zebrafish. A shared mechanism for pineal-specific and circadian gene expression. J. Biol. Chem. 280, 11544-11551.

Bhandarkar, S.S., Jaconi, M., Fried, L.E., Bonner, M.Y., Lefkove, B., Govindarajan, B., Perry, B.N., Parhar, R., Mackelfresh, J., Sohn, A., et al. (2009). Fulvene- 5 potently inhibits NADPH oxidase 4 and blocks the growth of endothelial tumors in mice. J. Clin. Invest. 119, 2359-2365.

Boutin, A.T., Weidemann, A., Fu, Z., Mesropian, L., Gradin, K., Jamora, C. Wiesener, M., Eckardt, K.U., Koch, C.J., Ellies, L.G., et al. (2008). Epidermal sensing of oxygen is essential for systemic hypoxic response. Cell 133, 223-234.

Bräutigam, L., Schütte, L.D., Godoy, J.R., Prozorovski, T., Gellert, M., Hauptmann, G., Holmgren, A., Lillig, C.H., and Berndt, C. (2011). Vertebrate-specific glutaredoxin is essential for brain development. Proc. Natl. Acad. Sci. USA 108, 20532-20537.

Cao, R., Jensen, L.D., Söll, I., Hauptmann, G., and Cao, Y. (2008). Hypoxiainduced retinal angiogenesis in zebrafish as a model to study retinopathy. PLoS ONE 3, e2748.

Cao, Y. (2009a). Positive and negative modulation of angiogenesis by VEGFR1 ligands. Sci. Signal. 2, re1.
Cao, Y. (2009b). Tumor angiogenesis and molecular targets for therapy. Front. Biosci. 14, 3962-3973.

Cao, Y., and Langer, R. (2010). Optimizing the delivery of cancer drugs that block angiogenesis. Sci. Transl. Med. 2, ps3.

Cao, Z., Jensen, L.D., Rouhi, P., Hosaka, K., Länne, T., Steffensen, J.F., Wahlberg, E., and Cao, Y. (2010). Hypoxia-induced retinopathy model in adult zebrafish. Nat. Protoc. 5, 1903-1910.

Cao, Y., Arbiser, J., D'Amato, R.J., D'Amore, P.A., Ingber, D.E., Kerbel, R., Klagsbrun, M., Lim, S., Moses, M.A., Zetter, B., et al. (2011). Forty-year journey of angiogenesis translational research. Sci. Transl. Med. 3, 114rv113.

Carmeliet, P., Ferreira, V., Breier, G., Pollefeyt, S., Kieckens, L., Gertsenstein M., Fahrig, M., Vandenhoeck, A., Harpal, K., Eberhardt, C., et al. (1996). Abnormal blood vessel development and lethality in embryos lacking a single VEGF allele. Nature 380, 435-439.

Caunt, M., Mak, J., Liang, W.C., Stawicki, S., Pan, Q., Tong, R.K., Kowalski, J., Ho, C., Reslan, H.B., Ross, J., et al. (2008). Blocking neuropilin-2 function inhibits tumor cell metastasis. Cancer Cell 13, 331-342.

Dahl Ejby Jensen, L., Cao, R., Hedlund, E.M., Söll, I., Lundberg, J.O., Hauptmann, G., Steffensen, J.F., and Cao, Y. (2009). Nitric oxide permits hypoxia-induced lymphatic perfusion by controlling arterial-lymphatic conduits in zebrafish and glass catfish. Proc. Natl. Acad. Sci. USA 106 18408-18413.

Eriksson, A., Cao, R., Pawliuk, R., Berg, S.M., Tsang, M., Zhou, D., Fleet, C. Tritsaris, K., Dissing, S., Leboulch, P., and Cao, Y. (2002). Placenta growth factor-1 antagonizes VEGF-induced angiogenesis and tumor growth by the formation of functionally inactive PIGF-1/VEGF heterodimers. Cancer Cell 1 , 99-108.

Ferrara, N., Carver-Moore, K., Chen, H., Dowd, M., Lu, L., O'Shea, K.S. Powell-Braxton, L., Hillan, K.J., and Moore, M.W. (1996). Heterozygous embryonic lethality induced by targeted inactivation of the VEGF gene. Nature $380,439-442$.

Ferrara, N., Gerber, H.P., and LeCouter, J. (2003). The biology of VEGF and its receptors. Nat. Med. 9, 669-676.

Folkman, J. (1995). Angiogenesis in cancer, vascular, rheumatoid and other disease. Nat. Med. 1, 27-31.

Folkman, J. (2007). Angiogenesis: an organizing principle for drug discovery? Nat. Rev. Drug Discov. 6, 273-286.

Gale, N.W., Dominguez, M.G., Noguera, I., Pan, L., Hughes, V., Valenzuela, D.M., Murphy, A.J., Adams, N.C., Lin, H.C., Holash, J., et al. (2004). Haploinsufficiency of delta-like 4 ligand results in embryonic lethality due to major defects in arterial and vascular development. Proc. Natl. Acad. Sci. USA $101,15949-15954$.

Haddon, C., Jiang, Y.J., Smithers, L., and Lewis, J. (1998). Delta-Notch signalling and the patterning of sensory cell differentiation in the zebrafish ear: evidence from the mind bomb mutant. Development 125, 4637-4644.

Hellström, M., Phng, L.K., Hofmann, J.J., Wallgard, E., Coultas, L., Lindblom, P., Alva, J., Nilsson, A.K., Karlsson, L., Gaiano, N., et al. (2007). Dll4 signalling through Notch1 regulates formation of tip cells during angiogenesis. Nature 445, 776-780.

Hrushesky, W.J., Lester, B., and Lannin, D. (1999). Circadian coordination of cancer growth and metastatic spread. Int. J. Cancer 83, 365-373.

Hunt, T., and Sassone-Corsi, P. (2007). Riding tandem: circadian clocks and the cell cycle. Cell 129, 461-464.

Jung, H., Choe, Y., Kim, H., Park, N., Son, G.H., Khang, I., and Kim, K. (2003) Involvement of CLOCK:BMAL1 heterodimer in serum-responsive mPer1 induction. Neuroreport 14, 15-19.

Kerbel, R., and Folkman, J. (2002). Clinical translation of angiogenesis inhibitors. Nat. Rev. Cancer 2, 727-739.

Kimmel, C.B., Ballard, W.W., Kimmel, S.R., Ullmann, B., and Schilling, T.F. (1995). Stages of embryonic development of the zebrafish. Dev. Dyn. 203 , 253-310. 
Lawson, N.D., and Weinstein, B.M. (2002). In vivo imaging of embryonic vascular development using transgenic zebrafish. Dev. Biol. 248, 307-318.

Lee, S.L., Rouhi, P., Dahl Jensen, L., Zhang, D., Ji, H., Hauptmann, G., Ingham, P., and Cao, Y. (2009). Hypoxia-induced pathological angiogenesis mediates tumor cell dissemination, invasion, and metastasis in a zebrafish tumor model. Proc. Natl. Acad. Sci. USA 106, 19485-19490.

Makino, Y., Cao, R., Svensson, K., Bertilsson, G., Asman, M., Tanaka, H., Cao, Y., Berkenstam, A., and Poellinger, L. (2001). Inhibitory PAS domain protein is a negative regulator of hypoxia-inducible gene expression. Nature 414 , 550-554.

Nagy, J.A., Dvorak, A.M., and Dvorak, H.F. (2007). VEGF-A and the induction of pathological angiogenesis. Annu. Rev. Pathol. 2, 251-275.

Nasevicius, A., Larson, J., and Ekker, S.C. (2000). Distinct requirements for zebrafish angiogenesis revealed by a VEGF-A morphant. Yeast 17, 294-301. Noguera-Troise, I., Daly, C., Papadopoulos, N.J., Coetzee, S., Boland, P., Gale, N.W., Lin, H.C., Yancopoulos, G.D., and Thurston, G. (2006). Blockade of DIl4 inhibits tumour growth by promoting non-productive angiogenesis. Nature 444, 1032-1037.

Pan, Q., Chanthery, Y., Liang, W.C., Stawicki, S., Mak, J., Rathore, N., Tong, R.K., Kowalski, J., Yee, S.F., Pacheco, G., et al. (2007). Blocking neuropilin1 function has an additive effect with anti-VEGF to inhibit tumor growth. Cancer Cell 11, 53-67.

Panda, S., Hogenesch, J.B., and Kay, S.A. (2002). Circadian rhythms from flies to human. Nature 417, 329-335.

Ridgway, J., Zhang, G., Wu, Y., Stawicki, S., Liang, W.C., Chanthery, Y., Kowalski, J., Watts, R.J., Callahan, C., Kasman, I., et al. (2006). Inhibition of DII4 signalling inhibits tumour growth by deregulating angiogenesis. Nature 444, 1083-1087.

Rouhi, P., Jensen, L.D., Cao, Z., Hosaka, K., Länne, T., Wahlberg, E., Steffensen, J.F., and Cao, Y. (2010). Hypoxia-induced metastasis model in embryonic zebrafish. Nat. Protoc. 5, 1911-1918.

Schultz, T.F., and Kay, S.A. (2003). Circadian clocks in daily and seasonal control of development. Science 301, 326-328.

Sehgal, A., and Mignot, E. (2011). Genetics of sleep and sleep disorders. Cell 146, 194-207.

Siekmann, A.F., and Lawson, N.D. (2007). Notch signalling limits angiogenic cell behaviour in developing zebrafish arteries. Nature 445, 781-784.
Soker, S., Takashima, S., Miao, H.Q., Neufeld, G., and Klagsbrun, M. (1998). Neuropilin-1 is expressed by endothelial and tumor cells as an isoformspecific receptor for vascular endothelial growth factor. Cell 92, 735-745.

Stainier, D.Y., Weinstein, B.M., Detrich, H.W., 3rd, Zon, L.I., and Fishman, M.C. (1995). Cloche, an early acting zebrafish gene, is required by both the endothelial and hematopoietic lineages. Development 121, 3141-3150.

Stevens, R.G., Hansen, J., Costa, G., Haus, E., Kauppinen, T., Aronson, K.J., Castaño-Vinyals, G., Davis, S., Frings-Dresen, M.H., Fritschi, L., et al. (2011). Considerations of circadian impact for defining 'shift work' in cancer studies: IARC Working Group Report. Occup. Environ. Med. 68, 154-162.

Stone, E.M. (2006). A very effective treatment for neovascular macular degeneration. N. Engl. J. Med. 355, 1493-1495.

Triqueneaux, G., Thenot, S., Kakizawa, T., Antoch, M.P., Safi, R., Takahashi, J.S., Delaunay, F., and Laudet, V. (2004). The orphan receptor Rev-erbalpha gene is a target of the circadian clock pacemaker. J. Mol. Endocrinol. 33, 585-608.

Wang, H. (2008a). Comparative analysis of period genes in teleost fish genomes. J. Mol. Evol. 67, 29-40.

Wang, H. (2008b). Comparative analysis of teleost fish genomes reveals preservation of different ancient clock duplicates in different fishes. Mar. Genomics 1, 69-78.

Wang, H. (2009). Comparative genomic analysis of teleost fish bmal genes. Genetica 136, 149-161.

Vatine, G., Vallone, D., Appelbaum, L., Mracek, P., Ben-Moshe, Z., Lahiri, K., Gothilf, Y., and Foulkes, N.S. (2009). Light directs zebrafish period2 expression via conserved D and E boxes. PLoS Biol. 7, e1000223.

Xue, Y., Cao, R., Nilsson, D., Chen, S., Westergren, R., Hedlund, E.M., Martijn, C., Rondahl, L., Krauli, P., Walum, E., et al. (2008). FOXC2 controls Ang-2 expression and modulates angiogenesis, vascular patterning, remodeling, and functions in adipose tissue. Proc. Natl. Acad. Sci. USA 105, 10167-10172. Xue, Y., Lim, S., Yang, Y., Wang, Z., Jensen, L.D., Hedlund, E.M., Andersson, P., Sasahara, M., Larsson, O., Galter, D., et al. (2012). PDGF-BB modulates hematopoiesis and tumor angiogenesis by inducing erythropoietin production in stromal cells. Nat. Med. 18, 100-110.

Ziv, L., Levkovitz, S., Toyama, R., Falcon, J., and Gothilf, Y. (2005). Functional development of the zebrafish pineal gland: light-induced expression of period2 is required for onset of the circadian clock. J. Neuroendocrinol. 17, 314-320. 


\section{EXTENDED DISCUSSION}

Period2 is markedly downregulated under DD conditions and downregulation of this negative regulator of angiogenesis may in part explain why DD exposure did not significantly inhibit developmental angiogenesis in zebrafish embryos. In contrast to LL exposure, zebrafish VEGF levels retained rhythmicity under DD exposure during our experimental periods, although prolonged DD exposure may lead to more severe disruption of the circadian rhythm (Cahill, 2002; Carr et al., 2006; Tamai et al., 2005, 2007). These findings further support differential regulation of clock genes and VEGF expression under LL and DD conditions.

Other independent studies also support the fact that LL and DD conditions differentially regulate Period2 expression (Pando et al., 2001; Vatine et al., 2009). Although our findings demonstrated regulation of developmental angiogenesis by clock genes, we cannot completely exclude the possibility of a circadian clock-independent mechanism underlying the Bmal1-modulated angiogenesis. This possibility warrant further mechanistic studies using zebrafish and perhaps mammalian genetic models.

\section{EXTENDED EXPERIMENTAL PROCEDURES}

\section{Zebrafish Strains and Husbandry}

Zebrafish were kept at Södertörns University, Huddinge, Sweden; or at the Department of Medical Biochemistry and Biophysics, Karolinska Institute, Stockholm, Sweden. All embryos were produced by natural matings, and maintained in standard $0.3 \times$ Danieau's or E3 embryo medium at $28.5^{\circ} \mathrm{C}$. All experiments were performed in accordance with ethical permissions granted by the North Stockholm Experimental Animal Ethical Committee, Sweden.

\section{Light Exposure}

Embryos were exposed to constant cold-light (1800 Lux), constant darkness, or a light-dark shift schedule (12-h light-dark cycle). Under these conditions, zebrafish embryos were raised at identical temperature in all three conditions, i.e., $28.5^{\circ} \mathrm{C}$. The embryos were kept at a density of 50 or less in approximately $30 \mathrm{ml}$ medium in a $10 \mathrm{~cm}$ bacterial culture plate placed in the running water on each shelf.

\section{Morpholino Treatment}

Morpholinos used in this study include include: bmal1a-MO (5'-TATCCATTCTTTGGTCTGCCATTAG-3'); bmal1a-MIS-MO (5'-TATCGATTGTTTGGTCTGCGATTAG-3'); bmal2-MO (5'-CAGATTCATTCCAGGTTGTCCAT-3'); period2-MO (5'-GGTCTTCA GACATCGGACTTGGGTT-3'); period2-MIS-MO (5'-GGTGTTCACAGATCCGACTTCGGTT-3'); clock3-MO (5'-ATGATAACTCGG TCTCATGGATCA-3'); vegfaa-MO (5'-GTATCAAATAAACAACCAAGTTCAT-3'); rbpsuh-MO (5'-CAAACTTCCCTGTCACAACA GGCGC-3'); and standard scrambled control-MO (5'-CCTCTTACCTCAGTTACAATTTATA-3'). These morpholinos have been previously used in other studies (Nasevicius et al., 2000; Siekmann and Lawson, 2007; Triqueneaux et al., 2004; Ziv et al., 2005) and were prepared at $3 \mathrm{mM}$ stocks that were subsequently diluted in nuclease-free water. Approximately $1 \mathrm{nl}$ was injected in the yolk of each embryo at the 1-2 cell stage. The injected embryos were then placed in fresh Danieu's medium or E3 medium, followed by exposure to LD, LL or DD as indicated above.

\section{Assessment of Morpholino Efficiency}

Sequences encoding the bmal1a, bmal2, period 2 and clock3 open reading frames including the upstream attachment site of each morpholino were amplified from whole zebrafish cDNA using the following primers: zebrafish bmal1a Forward: $5^{\prime}-C A C A C A G G A$ TCCTCTGTGCGGATGATCTAATGGC-3' and Reverse: 5'-CACACAGAATTCCAAAGGCCAGGGCAGGTCAC-3'; zebrafish bmal2 Forward: 5'-CACACAGGATCCGGTTTGATGACCCCCAC-3' and Reverse: 5'-CACACAGAATTCGAGAGACCAGTGCATCTC-3'; zebrafish period2 Forward: 5'-CACACAGGATCCGGTGATTTGTTCGTGTTTTAC-3' and Reverse: 5'-CACACAGAATTCGGTGTCTG GACCGGG-3'; zebrafish clock3 Forward: 5'-CACACAGGATCCGGCAGGTCCTCTTCACC-3' and Reverse: 5'-CACACACTCGAGC TCGAGCTGAGACTGGGCACTAGC-3'. These were cloned into the pCS2-C-Myc vector to generate C-terminal myc-tag fusion constructs via BamHI/EcoRI or BamH1/Xhol sites, respectively. Capped mRNA was generated using the mMessage/Machine kit (Ambion) according to the manufacture's procedure. Each zebrafish embryo was injected at the 1 cell stage with $15 \mathrm{ng}$ capped mRNA in presence or absence of $0.6 \mathrm{mM}$ of the corresponding morpholino in a total volume of $1 \mathrm{nl}$. Myc-fusion proteins were detected in the presence or absence of morpholino by immunohistochemistry with a 9E10 monoclonal anti-myc antibody (Sigma) in conjunction with NBT/BCIP staining using an alkaline phosphatase-conjugated goat anti-mouse lgG secondary antibody (Sigma).

Pharmacological Inhibition of VEGFRs or Notch Signaling

ZN323881, sunitinib malate (LC Laboratories, MA) and DAPT (Sigma) were dissolved in DMSO. Embryos exposed to the same concentration of DMSO in the embryo medium were used as a vehicle control.

\section{RT-PCR}

Zebrafish embryos (20-50 embryos/group) were dispensed into $1 \mathrm{ml}$ TRIzol (QIAGEN), followed by homogenization for $30 \mathrm{~s}$. Total RNA $(1 \mu \mathrm{g})$ from each sample was reverse-transcribed using a RevertAid H minus First Strand cDNA Synthesis Kit (Fermentas). 
Reverse transcription was performed at $42^{\circ} \mathrm{C}$ for $60 \mathrm{~min}$, followed by $70^{\circ} \mathrm{C}$ for $5 \mathrm{~min}$ to inactivate the enzyme activity. Samples were stored at $-20^{\circ} \mathrm{C}$ or directly subjected to RT-PCR. RT-PCR was performed in a $20 \mu$ reaction mixture containing DreamTaq Green PCR Master Mix (Thermo Scientific), $500 \mathrm{nM}$ forward and reverse primers, and $1 \mu \mathrm{l}$ of cDNA. The RT-PCR protocol was executed for 30 cycles and each cycle consisted of denaturation at $95^{\circ} \mathrm{C}$ for $30 \mathrm{~s}$, annealing at $60^{\circ} \mathrm{C}$ for $30 \mathrm{~s}$, and extension at $72^{\circ} \mathrm{C}$ for 1 min. In the initial cycle, samples were incubated at $95^{\circ} \mathrm{C}$ for $2 \mathrm{~min}$. The primer pairs specific for various genes used in our experiments are: zebrafish period2, Forward: 5'-AAGCCAAGACGCAGAAAGA-3', and Reverse: 5'-CAAGAATGCCAAGTTTGTGG-3'; zebrafish bmal1a Forward: 5'-GCTGTGGCTCCAGTCCTTTA-3', and Reverse: 5'-TTCATCATTACTGGGGCTGCTGGA-3'; zebrafish vegfaa, Forward: 5'-AGAAAGAAAACCACTGTGAG-3', and Reverse: 5'-AGGAATGTTCTTCCTTAGGT-3'; zebrafish $\beta$-actin, Forward: 5'-TTGTAACC AACTGGGACGATATGG-3', and Reverse: 5'- GATCTTGATCTTCATGGTGCTAGG - $3^{\prime}$.

\section{Zebrafish VEGF-Luciferase Construction and Promoter Deletion}

A $2.5 \mathrm{~kb}$ fragment (nucleotide position -2500 to -4 ; zvegf ptr $2.5 \mathrm{~kb}$ ) of the promoter for the zebrafish vegfab gene was generated by PCR from zebrafish genomic DNA using the following primers: Forward: 5'-AGCTCGCTTTCTCTGTCATT-3'; and Reverse: 5'-TTAACGAACTGCTTCAGCTG-3'. The promoter fragments of $1090 \mathrm{bp}(-1093$ to -4 ; zvegf ptr $1 \mathrm{~kb})$ and $857-\mathrm{bp}(-860$ to -4 ; zvegf ptr $0.86 \mathrm{~kb}$ ) were amplified from the $2.5 \mathrm{~kb}$ fragment using the following paired primers: Forward: $5^{\prime}-\mathrm{GTTGTGATTTCCTGGGC}$ TAG-3'; and Reverse: 5'-TTAACGAACTGCTTCAGCTG-3'; and Forward: 5'-GCCGATGATATTCAAACTGG-3'; and Reverse: 5'-TTAACGAACTGCTTCAGCTG-3'; respectively. zvegf ptr $0.86 \mathrm{~kb} \Delta$ was generated by PCR using zvegf ptr $0.86 \mathrm{~kb}$ as a template and the following mutant synthetic oligonucleotide primers: Forward: 5'-ATAATAAGTTTATTGTTATTTGTAAAGCATATGCAAA TGC-3'; and Reverse: 5'-GCATTTGCATATGCTTTACAAATAACAATAAACTTATTAT-3'. The above amplified promoter fragments were cloned into the pGL3-Basic vector (Promega, Madison, Wisconsin), according to the manufacturer's instructions, to generate zvegf promoter-luciferase reporter constructs.

\section{Promoter Activity Assay}

Zebrafish embryos were injected at the 1-2 cell stage with $1 \mathrm{nl}$ of $100 \mathrm{ng} / \mathrm{nl}$ human vegf promoter-luciferase, mouse bmal1 promoterluciferase (kindly provided by Dr. Yagita, Nagoya University, Japan), or zebrafish vegfab promoter-luciferase, followed by immediate transfer to LD or LL conditions. Five embryos were harvested at each indicated time point from each group and were placed for $30 \mathrm{~min}$ in $100 \mu \mathrm{l}$ of a passive lysis buffer (Vector). The lyzed solution was centrifuged at $10000 \mathrm{rpm}$ for $5 \mathrm{~min}$. $20 \mu \mathrm{l}$ of the supernatant were added to $100 \mu \mathrm{l}$ of a luciferase activity reagent from the dual luciferase kit (Vector). The values were recorded from a luminometer and were normalized to the total amount of protein in the sample as determined by the Dc protein assay according to the manufacturer's instructions (BioRad, Sweden).

\section{Quantitative Real-Time PCR}

Primer sequences are as follows: zebrafish vegfaa Forward: 5'-GAGAGCCAGCGACTCACCGCAACAC-3'; and Reverse: 5'-GTT CGCTCGATCATCATCTTGGC -3'; zebrafish bmal1a Forward: 5'-GCAACCGCAAGAGGAAAGGCAGTG-3'; and Reverse: 5'-CTCG TCGTTACTGGGGCTGCTTGA-3'; zebrafish period2 Forward: 5'-AAGCCAAGACGCAGAAAGAG-3'; and Reverse: 5'-CCACAAA CTTGGCATTCTTG-3'; zebrafish $\beta$-actin Forward: 5'-TTGTAACCAACTGGGACGATATGG-3'; and Reverse: 5'-GATCTTGATCTTCA TGGTGCTAGG $-3^{\prime}$.

\section{Chromatin Immunoprecipitation Assay}

Mouse fibroblast cells were used for the EZ-ChIP chromatin immunoprecipitation assay (Millipore, Billerica, MA) as previously described (Xue et al., 2012). In brief, $100 \mu \mathrm{l}$ of sonicated chromatin was used for each immunoprecipitation reaction. A rabbit antiBmal1 antibody (2.5 $\mu$ g per sample, Abcam, Cambridge, UK) and a non-immune rabbit lgG (2.5 $\mu \mathrm{g}$ per sample, Millipore, Billerica, MA) were used for immunoprecipitation. The purified DNA was used for quantitative PCR analysis. To amplify the mouse vegf promoter fragment containing E-boxes $1-3$, the following primers were used: Forward: $5^{\prime}-$ GTCTACAGCCTCTGTTATGCCAC- ${ }^{\prime}$ and Reverse: $5^{\prime}-G G T T T C C T C A C C T G A T C C T G-3{ }^{\prime}$. For amplification of a fragment containing E-boxes 4 and 5 , the following primers were used: Forward: 5'-CCGCACTCAAGAATCAACTC-3' and Reverse: 5'-GCAGGGACGTATGAGGATAG-3'. An E-box-less fragment from the mouse vegf gene (4537- 4813 downstream from the initial codon) was used as a negative control, which was amplified using primers: Forward: 5'-GTACACTCACACCAGCCCATC- $3^{\prime}$ and Reverse: 5'-CCCATGACGATGCTTCTCTTC-3'. A mouse period 1 fragment, known to bind to Bmal1, was used as a positive control, and this fragment was amplified using primers: Forward: 5'-GCAGTATTGGGTAAGTGTCGTC-3' and Reverse: 5'-GCAACCATTGGCATTTCAGAAC-3'.

\section{Image Analysis}

The 4 ISVs posterior to the fusion of two arms of the common cardinal vein into the posterior cardinal vein were measured as mean determinants of ISV length. The SIV with the largest area was used as the SIV area in each embryo. At least 12 embryos were analyzed per group and at least 3 separate experiments were performed. Images were subsequently analyzed in either Photoshop CS4 (Adobe) or ImageJ. 


\section{SUPPLEMENTAL REFERENCES}

Cahill, G.M. (2002). Clock mechanisms in zebrafish. Cell Tissue Res. 309, 27-34

Carr, A.J., Tamai, T.K., Young, L.C., Ferrer, V., Dekens, M.P., and Whitmore, D. (2006). Light reaches the very heart of the zebrafish clock. Chronobiol. Int. 23, 91-100.

Pando, M.P., Pinchak, A.B., Cermakian, N., and Sassone-Corsi, P. (2001). A cell-based system that recapitulates the dynamic light-dependent regulation of the vertebrate clock. Proc. Natl. Acad. Sci. USA 98, 10178-10183.

Tamai, T.K., Carr, A.J., and Whitmore, D. (2005). Zebrafish circadian clocks: cells that see light. Biochem. Soc. Trans. 33 , $962-966$.

Tamai, T.K., Young, L.C., and Whitmore, D. (2007). Light signaling to the zebrafish circadian clock by Cryptochrome 1a. Proc. Natl. Acad. Sci. USA 104, 14712-14717. 

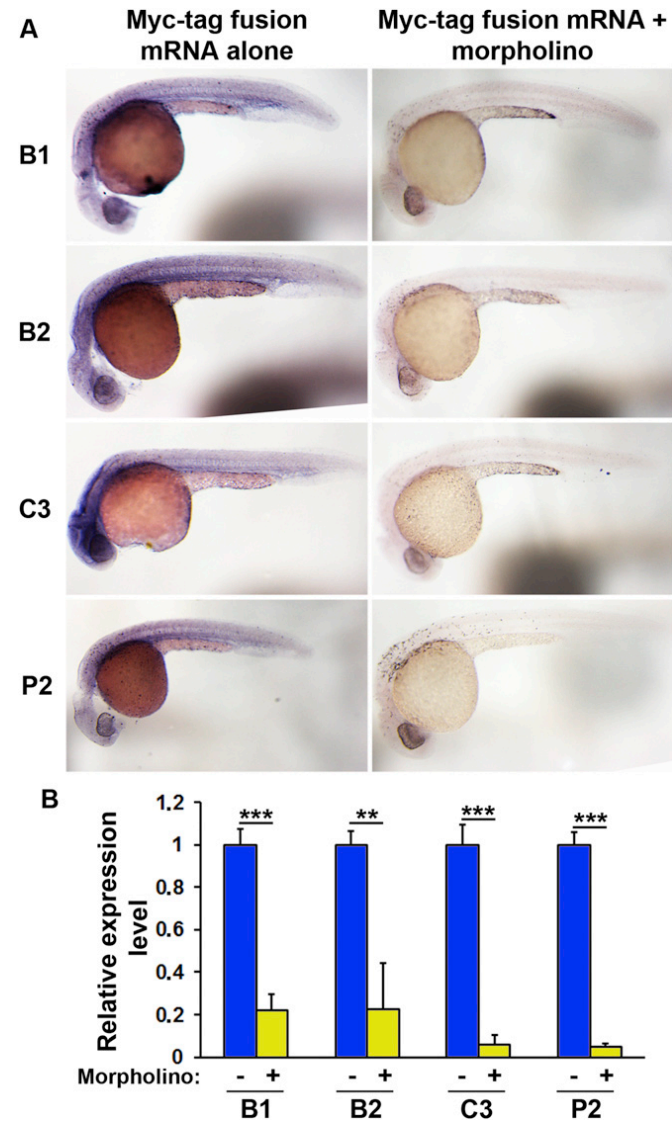

Figure S1. Knockdown Efficiency of Morpholinos, Related to Figure 2

(A) Immunohistochemistry of clock gene mRNA-injected zebrafish embryos with or without morpholinos. The translated proteins were detected using an anti-myc tag antibody, followed by detection with an alkaline phosphatase-conjugated secondary antibody. The translated positive signals were revealed using the NBT/ BCIP technique. Blue color shows positive signals of the translated proteins.

(B) Quantifications of average clock protein levels \pm SEM in mRNA-injected zebrafish embryos (18-24 embryos/group). 

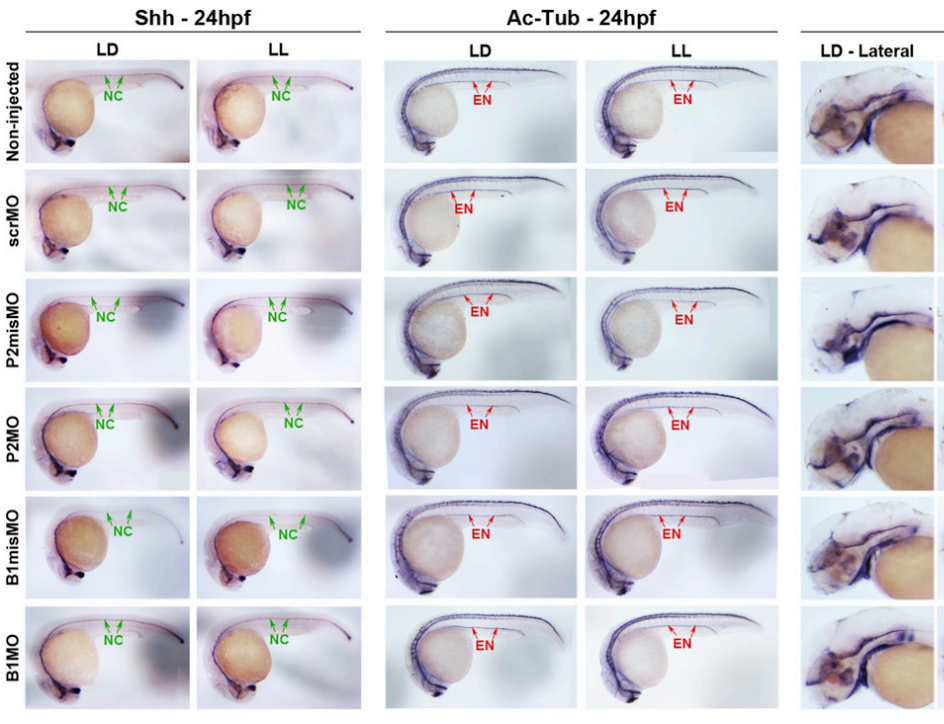

Shh - 48hpf
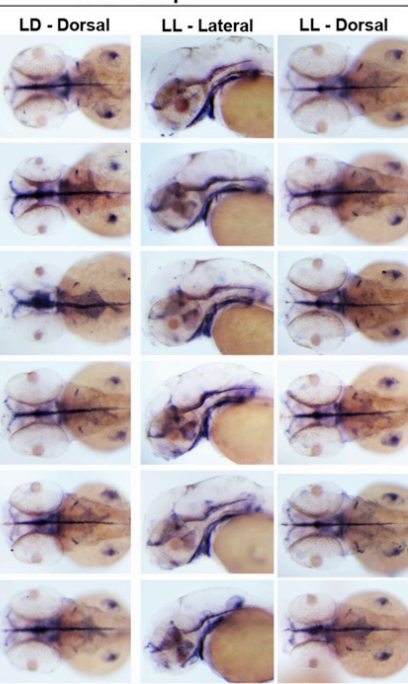

Figure S2. Morphology and Expression Patterns of Sonic Hedgehog and Acetylated Tubulin in Zebrafish Embryos, Related to Figures 1 and 2 Zebrafish embryos at the 1-2 cell stage received injections of specific morpholinos targeting Bmal1 (B1MO), or Period2 (P2MO) (0.2 pmol/embryo) and subsequently raised under LL or LD conditions. Scrambled morpholino (scrMO, 0.2 pmol/embryo), 5 base-pair mismatch bmal1 (B1misMO, 0.2 pmol/embryo), and 5 base-pair mismatch period2 (P2misMO, 0.2 pmol/embryo) were used as controls. Expression of sonic hedgehog (shh) at 24 or 48 hpf was detected by in situ hybridization. Expression of acetylated tubulin (Ac-Tub) was detected by immunohistochemistry using a specific anti Ac-Tub antibody. NC = notochord; $\mathrm{EN}=$ enteric nerve. 

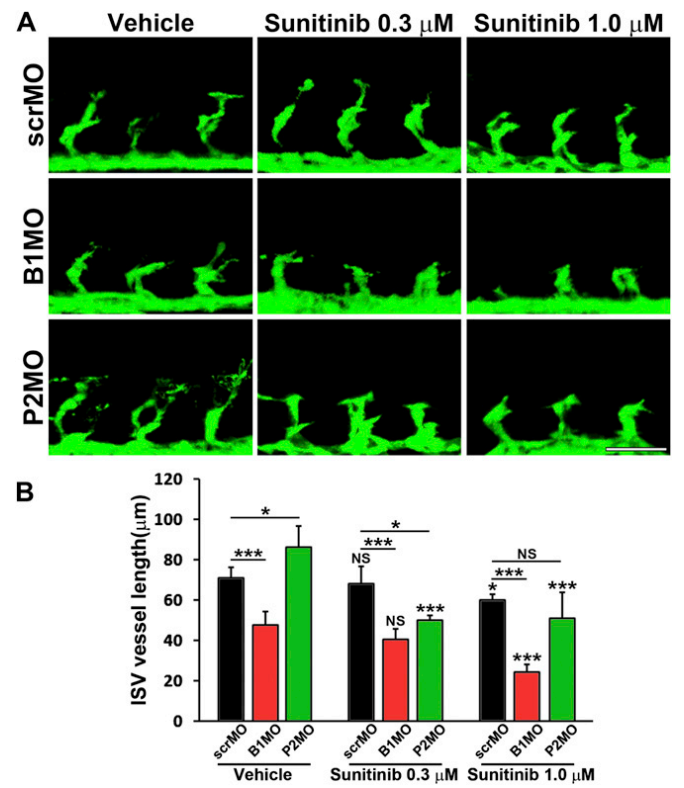

Figure S3. Additive Effect on Suppression of Developmental Angiogenesis by a Bmal1 Morpholino and Sunitinib, Related to Figure 3 (A) $T g$ (Fli1:egfp $)^{y 1}$ zebrafish embryos were injected with scrambled control (scrMO), bmal1 (B1MO) or period2 (P2MO) mopholinos (0.2 pmol/embryo), and placed in medium with either the vehicle buffer or sunitinib. ISV development was investigated at $24 \mathrm{hpf}$. Scale bar $=50 \mu \mathrm{m}$.

(B) Quantification of the average ISV length \pm SEM of 24-hpf embryos ( $n=4$ embryos/group). 
A

hpf: 96102108114120126132138144

B1

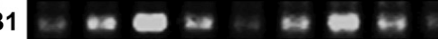

VEGF

$\beta$-actin

B

VEGF
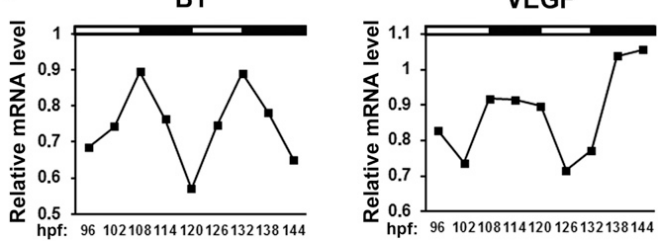

Figure S4. RT-PCR Analysis of Circadian Rhythm of Zebrafish bmal1 and vegf Expression, Related to Figure 3

(A) RT-PCR analysis of zebrafish bmal1 and vegf expression under LD conditions (a pooled sample of 25 zebrafish embryos/time point). Standard loading was indicated by $\beta$-actin expression.

(B) Densitometric quantification of average \pm SEM bmal1 and vegf expressions shown in (A). 